ANALYSIS \& PDE Volume $6 \quad$ No. $3 \quad 2013$ VOIKRR SCHLUE
DECAY OF HNEAR WAYES ON HIGHER-DIMENSIONAL SCHWARZSCHIID BLACK HOLES 


\title{
DECAY OF LINEAR WAVES ON HIGHER-DIMENSIONAL SCHWARZSCHILD BLACK HOLES
}

\author{
VOLKER SCHLUE
}

We consider solutions to the linear wave equation on higher dimensional Schwarzschild black hole spacetimes and prove robust nondegenerate energy decay estimates that are in principle required in a nonlinear stability problem. More precisely, it is shown that for solutions to the wave equation $\square_{g} \phi=0$ on the domain of outer communications of the Schwarzschild spacetime manifold $\left(\mathcal{M}_{m}^{n}, g\right)$ (where $n \geq 3$ is the spatial dimension, and $m>0$ is the mass of the black hole) the associated energy flux $E[\phi]\left(\Sigma_{\tau}\right)$ through a foliation of hypersurfaces $\Sigma_{\tau}$ (terminating at future null infinity and to the future of the bifurcation sphere) decays, $E[\phi]\left(\Sigma_{\tau}\right) \leq C D / \tau^{2}$, where $C$ is a constant depending on $n$ and $m$, and $D<\infty$ is a suitable higher-order initial energy on $\Sigma_{0}$; moreover we improve the decay rate for the first-order energy to $E\left[\partial_{t} \phi\right]\left(\Sigma_{\tau}^{R}\right) \leq C D_{\delta} / \tau^{4-2 \delta}$ for any $\delta>0$, where $\Sigma_{\tau}^{R}$ denotes the hypersurface $\Sigma_{\tau}$ truncated at an arbitrarily large fixed radius $R<\infty$ provided the higher-order energy $D_{\delta}$ on $\Sigma_{0}$ is finite. We conclude our paper by interpolating between these two results to obtain the pointwise estimate $|\phi|_{\Sigma_{\tau}^{R}} \leq C D_{\delta}^{\prime} / \tau^{\frac{3}{2}-\delta}$. In this work we follow the new physical-space approach to decay for the wave equation of Dafermos and Rodnianski (2010).

1. Introduction $\quad 516$

1A. Statement of the theorems 516

1B. Overview of the proof $\quad 520$

2. Global causal geometry of the higher-dimensional Schwarzschild solution 522

3. The redshift effect $\quad 526$

4. Integrated local energy decay $\quad 531$

4A. Radial multiplier vector fields 533

4B. High angular frequencies $\quad 537$

4C. Low angular frequencies and commutation $\quad 546$

4D. Boundary terms $\quad 554$

5. The decay argument $\quad 564$

5A. Uniform boundedness $\quad 564$

5B. Energy decay $\quad 566$

5C. Improved interior decay of the first-order energy $\quad 574$

6. Pointwise bounds $\quad 588$

Appendix A. Notation $\quad 593$

Appendix B. Formulas for reference $\quad 593$

Appendix C. Boundary integrals and Hardy inequalities $\quad 595$

Acknowledgements $\quad 599$

References $\quad 599$

MSC2010: 35L05, 35Q75, 58J45, 83C57.

Keywords: decay, wave equation, Schwarzschild black hole, spacetime, higher dimensions, mathematical general relativity. 


\section{Introduction}

The study of the wave equation on black hole spacetimes has generated considerable interest in recent years. This stems mainly from its role as a model problem for the nonlinear black hole stability problem [Dafermos and Rodnianski 2009a; 2012], and more recent advances in the analysis of linear waves [Dafermos and Rodnianski 2008].

In this paper we study the linear wave equation on higher-dimensional Schwarzschild black holes. The motivation for this problem lies - apart from the above mentioned relation to the nonlinear stability problem (which is expected to be simpler in the higher-dimensional case [Choquet-Bruhat et al. 2006]; for work on the 5-dimensional case under symmetry see also [Dafermos and Holzegel 2006; Holzegel 2010]) - on one hand in the purely mathematical curiosity of dealing with higher dimensions and on the other hand in its interest for theories of high energy physics [Emparan and Reall 2008].

In the philosophy of [Christodoulou and Klainerman 1993] it is understood that the resolution of the nonlinear stability problem requires an understanding of the linear equations in a sufficiently robust setting. In particular, we require a proof of the uniform boundedness and decay of solutions to the linear wave equation based on the method of energy currents, which (ideally) only uses properties of the spacetime that are stable under perturbations, and does not rely heavily on the specifics of the unperturbed metric (for an introduction in the context of black hole spacetimes see [Dafermos and Rodnianski 2008]). Correspondingly in this paper we establish on higher-dimensional Schwarzschild spacetime backgrounds boundedness and decay results analogous to the current state of the art in the $(3+1)$-dimensional case [Luk 2010].

The decay argument presented here departs from earlier work that either makes use of multipliers with weights in the temporal variable (notably [Christodoulou and Klainerman 1990; Blue and Sterbenz 2006; Andersson and Blue 2009; Dafermos and Rodnianski 2009b; Luk 2010]) which in one form or the other are due to Morawetz [1962], or that relies on the exact stationarity of the spacetime (such as [Ching et al. 1995; Tataru 2010; Donninger et al. 2012] based on Fourier analytic methods). Here we follow the new physical-space approach to decay of [Dafermos and Rodnianski 2010], which only uses multipliers with weights in the radial variable. Thus our work - especially the improvement of Section $5 \mathrm{C}$ - is of independent interest for the $(3+1)$-dimensional Schwarzschild and Minkowski case and also for a wider class of spacetimes including Kerr black hole exteriors.

1A. Statement of the theorems. We consider solutions to the wave equation

$$
\square_{g} \phi=0
$$

on higher-dimensional Schwarzschild black hole spacetimes; these backgrounds are a family of $(n+1)$ dimensional Lorentzian manifolds $\left(\mathcal{M}_{m}^{n}, g\right)$ parametrized by the mass of the black hole $m>0(n \geq 3)$. They arise as spherically symmetric solutions of the vacuum Einstein equations, the governing equations of general relativity, and are discussed as such in Section 2; for the relevant concepts see also [Dafermos and Rodnianski 2008; Hawking and Ellis 1973].

More precisely, we consider solutions to (1-1) on the domain of outer communications $\mathcal{D}$ of $\mathcal{M}-$ which comprises the exterior up to and including the event horizons of the black hole — with initial data 


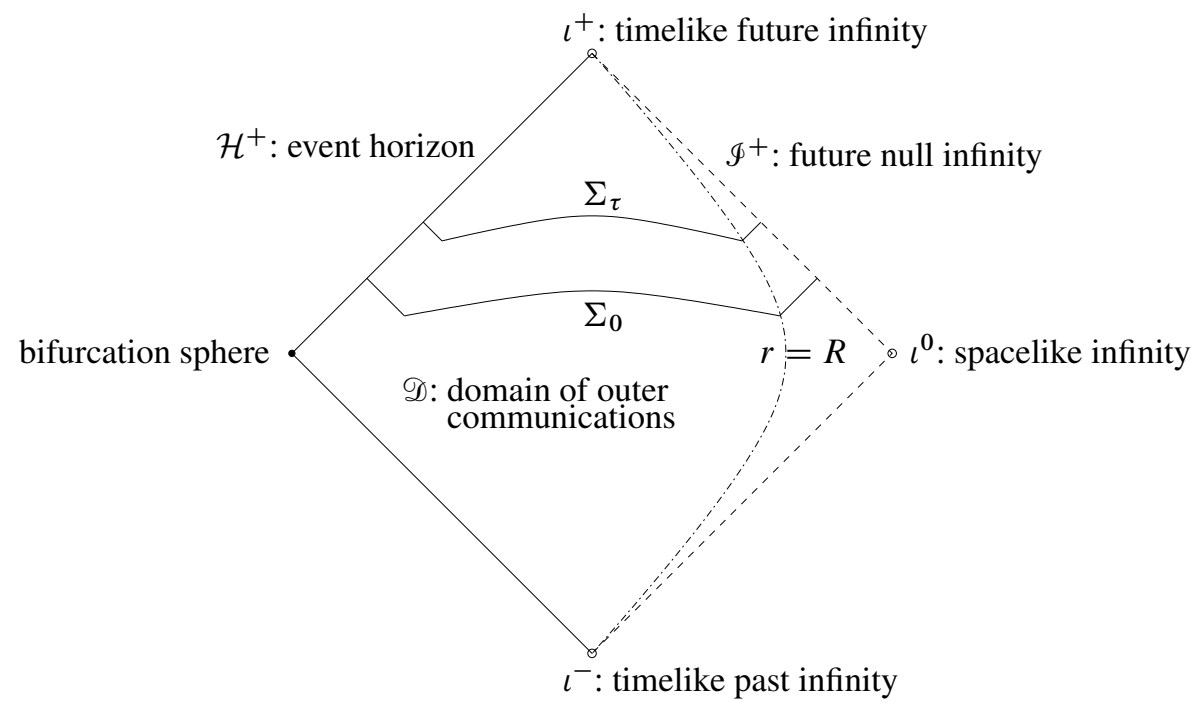

Figure 1. The hypersurface $\Sigma_{0}$ in the domain of outer communications $\mathcal{D}$.

prescribed on a hypersurface $\Sigma_{0}$ consisting of an incoming null segment crossing the event horizon to the future of the bifurcation sphere, a spacelike segment and an outgoing null segment emerging from a larger sphere of radius $R$ terminating at future null infinity; see Figure 1 (the exact parametrization which is chosen merely for technical reasons - is given in Section 4).

In the exterior of the black hole the metric $g$ takes the classical form in $(t, r)$-coordinates [Tangherlini 1963]:

$$
g=-\left(1-\frac{2 m}{r^{n-2}}\right) \mathrm{d} t^{2}+\left(1-\frac{2 m}{r^{n-2}}\right)^{-1} \mathrm{~d} r^{2}+r^{2} \dot{\gamma}_{n-1},
$$

where $r>\sqrt[n-2]{2 m}, t \in(-\infty, \infty)$, and $\dot{\gamma}_{n-1}$ denotes the standard metric on the unit $(n-1)$-sphere; however this coordinate system breaks down on the horizon $r=\sqrt[n-2]{2 m}$ and we shall for that reason introduce in Section 2 the global geometry of $\left(\mathcal{M}_{m}^{n}, g\right)$ using a double null foliation, from which we derive an alternative double null coordinate system for the exterior of the black hole:

$$
g=-4\left(1-\frac{2 m}{r^{n-2}}\right) \mathrm{d} u^{*} \mathrm{~d} v^{*}+r^{2} \dot{\gamma}_{n-1},
$$

the so-called Eddington-Finkelstein coordinates.

In this paper both the conditions on the initial data and the statements on the decay of the solutions are formulated using the concepts of energy and the energy momentum tensor associated to (1-1); in particular (see Section 1B and also Appendix B),

$$
T_{\mu \nu}[\phi]=\partial_{\mu} \phi \partial_{\nu} \phi-\frac{1}{2} g_{\mu \nu} \partial^{\alpha} \phi \partial_{\alpha} \phi
$$

The corresponding 1-contravariant-1-covariant tensor field fulfills the physical requirement that the linear transformation $-T: T \mathcal{M} \rightarrow T \mathcal{M}$ maps the hyperboloid of future-directed unit timelike vectors into the 
closure of the open future cone at each point. Physically,

$$
-T \cdot u \in T_{p} \mathcal{M}
$$

is the energy-momentum density relative to an observer at $p \in \mathcal{M}$ with 4-velocity $u \in T_{p} \mathcal{M}$, and it is for this reason that we refer to

$$
\varepsilon=g(T \cdot u, u)=T(u, u) \geq 0
$$

as the energy density at $p \in \mathcal{M}$ relative to the observer with 4-velocity $u \in T_{p} \mathcal{M}$. One may think of a spacelike hypersurface as a collection of locally simultaneous observers with a 4-velocity given by the normal. The hypersurfaces relative to which we establish energy decay are simply defined by $\Sigma_{\tau} \doteq \varphi_{\tau}\left(\Sigma_{0} \cap \mathcal{D}\right)$, where $\varphi_{\tau}$ denotes the 1-parameter group of isometries generated by $\frac{\partial}{\partial t}$. The energy flux through the hypersurface $\Sigma_{\tau}$ is then given by

$$
E[\phi]\left(\Sigma_{\tau}\right) \doteq \int_{\Sigma_{\tau}}\left(J^{N}[\phi], n_{\Sigma}\right)
$$

where $\left(J^{N}[\phi], n_{\Sigma}\right) \doteq T[\phi]\left(N, n_{\Sigma}\right), n_{\Sigma}$ is the normal ${ }^{1}$ to $\Sigma_{\tau}$ and $N$ is a timelike $\varphi_{\tau}$-invariant future directed vector field which is constructed in Section 3 for the purpose of turning $\varepsilon^{N} \doteq T(N, N)$ into a nondegenerate energy up to and including the horizon. Note that the energy $E[\phi]\left(\Sigma_{\tau}\right)$ in particular bounds a suitably defined $\dot{\mathrm{H}}^{1}$-norm on $\Sigma_{\tau}$.

The classes of solutions to (1-1) to which our results apply are formulated in terms of finite energy conditions on the initial data, for which purpose we list the following quantities:

$$
\begin{aligned}
D_{2}^{(2)}\left(\tau_{0}\right) \doteq & \left.\int_{\tau_{0}+R^{*}}^{\infty} \mathrm{d} v^{*} \int_{\mathbb{S}^{n-1}} \mathrm{~d} \mu_{\dot{\gamma}_{n-1}} \sum_{k=0}^{1} r^{2}\left(\frac{\partial\left(r^{\frac{n-1}{2}} \partial_{t}^{k} \phi\right)}{\partial v^{*}}\right)^{2}\right|_{u^{*}=\tau_{0}} \\
& +\int_{\Sigma_{\tau_{0}}}\left(\sum_{k=0}^{2} J^{N}\left[\partial_{t}^{k} \phi\right], n_{\Sigma}\right), \\
D_{5}^{(4-\delta)}\left(\tau_{0}\right) \doteq & \int_{\tau_{0}+R^{*}}^{\infty} \mathrm{d} v^{*} \int_{\mathbb{S}^{n-1}} \mathrm{~d} \mu_{\gamma_{n-1}}\left\{\sum_{k=0}^{1} r^{4-\delta}\left(\frac{\partial^{2}\left(r^{\frac{n-1}{2}} \partial_{t}^{k} \phi\right)}{\partial v^{* 2}}\right)^{2}\right. \\
& \left.+\sum_{k=0}^{4} r^{2}\left(\frac{\partial\left(r^{\frac{n-1}{2}} \partial_{t}^{k} \phi\right)}{\partial v^{*}}\right)^{2}+\sum_{k=0}^{3} \frac{n(n-1)}{\sum_{i=1}^{2}} r^{2}\left(\frac{\partial r^{\frac{n-1}{2}} \Omega_{i} \partial_{t}^{k} \phi}{\partial v^{*}}\right)^{2}\right)\left.\right|_{u^{*}=\tau_{0}} \\
& +\int_{\Sigma_{\tau_{0}}}\left(\sum_{k=0}^{5} J^{N}\left[\partial_{t}^{k} \phi\right]+\sum_{k=0}^{4} \frac{n(n-1)}{\sum_{i=1}^{2}} J^{N}\left[\Omega_{i} \partial_{t}^{k} \phi\right], n_{\Sigma}\right),
\end{aligned}
$$

\footnotetext{
${ }^{1}$ On spacelike segments of $\Sigma_{\tau}$ the vector $n_{\Sigma}$ is indeed timelike; however, on the null segments of the hypersurfaces $\Sigma_{\tau}$ the "normal" $n_{\Sigma}$ is in fact a null vector, but the notation is kept for convenience; see Appendix A.
} 


$$
\begin{aligned}
D_{7+\left[\frac{n}{2}\right]}^{(4-\delta)}\left(\tau_{0}\right) \doteq & \int_{\tau_{0}+R^{*}}^{\infty} \mathrm{d} v^{*} \int_{\mathbb{S}^{n-1}} \mathrm{~d} \mu_{\dot{\gamma}_{n-1}}\left\{\sum_{k=0}^{2} \sum_{|\alpha| \leq\left[\frac{n}{2}\right]+1} r^{4-\delta}\left(\frac{\partial^{2}\left(r^{\frac{n-1}{2}} \Omega^{\alpha} \partial_{t}^{k} \phi\right)}{\partial v^{* 2}}\right)^{2}\right. \\
& \left.+\sum_{k=0}^{5} \sum_{|\alpha| \leq\left[\frac{n}{2}\right]+1} r^{2}\left(\frac{\partial r^{\frac{n-1}{2}} \Omega^{\alpha} \partial_{t}^{k} \phi}{\partial v^{*}}\right)^{2}+\sum_{k=0|\alpha| \leq\left[\frac{n}{2}\right]+2}^{4} r^{2}\left(\frac{\partial r^{\frac{n-1}{2}} \Omega^{\alpha} \partial_{t}^{k} \phi}{\partial v^{*}}\right)^{2}\right\}\left.\right|_{u^{*}=\tau_{0}} \\
& +\int_{\Sigma_{\tau_{0}}}\left(\sum_{k=0}^{6} \sum_{|\alpha| \leq\left[\frac{n}{2}\right]+1} J^{N}\left[\Omega^{\alpha} \partial_{t}^{k} \phi\right]+\sum_{k=0|\alpha| \leq\left[\frac{n}{2}\right]+2} J^{N}\left[\Omega^{\alpha} \partial_{t}^{k} \phi\right], n_{\Sigma}\right) .
\end{aligned}
$$

Here $\Omega_{i}: i=1, \ldots, n(n-1) / 2$ are the generators of the spherical isometries of the spacetime $\mathcal{M}$, $\alpha$ is a multiindex, and for any radius $R$ we denote by $R^{*}$ the corresponding Regge-Wheeler radius (2-17). (See also Section 4B.)

Among the propositions on linear waves on higher-dimensional Schwarzschild black hole spacetimes proven in this paper, we wish to highlight the following conclusions ${ }^{2}$.

Theorem 1 (energy decay). Let $\phi$ be a solution of the wave equation $\square_{g} \phi=0$ on $\mathcal{D} \subset \mathcal{M}_{m}^{n}$, where $n \geq 3$ and $m>0$, with initial data prescribed on $\Sigma_{\tau_{0}}\left(\tau_{0}>0\right)$.

- If $D \doteq D_{2}^{(2)}\left(\tau_{0}\right)<\infty$ then there exists a constant $C(n, m)$ such that

$$
E[\phi]\left(\Sigma_{\tau}\right) \leq \frac{C D}{\tau^{2}} \quad\left(\tau>\tau_{0}\right)
$$

- Furthermore if for some $0<\delta<\frac{1}{2}$ and $R>\sqrt[n-2]{8 n m / \delta}$ also $D^{\prime} \doteq D_{5}^{(4-\delta)}\left(\tau_{0}\right)<\infty$ then there exists a constant $C(n, m, \delta, R)$ such that

$$
E\left[\partial_{t} \phi\right]\left(\Sigma_{\tau}^{\prime}\right) \leq \frac{C D^{\prime}}{\tau^{4-2 \delta}} \quad\left(\tau>\tau_{0}\right)
$$

where $\Sigma_{\tau}^{\prime} \doteq \Sigma_{\tau} \cap\{r \leq R\}$.

While each of these energy decay statements lend themselves to prove pointwise estimates for $\phi$ and $\partial_{t} \phi$ respectively (see Section 6), we would like to emphasize that, using the (refined) integrated local energy decay estimates of Section 4, an interpolation argument allows to improve the pointwise bound on $\phi$ directly in the interior ${ }^{3}$.

Theorem 2 (pointwise decay). Let $\phi$ be a solution of the wave equation as in Theorem 1. If for some $0<\delta<\frac{1}{4}, D \doteq D_{7+\left[\frac{n}{2}\right]}^{(4-\delta)}\left(\tau_{0}\right)<\infty\left(\tau_{0}>1\right)$ then there exists a constant $C(n, m, \delta, R)$ such that

$$
\left.r^{\frac{n-2}{2}}|\phi|\right|_{\Sigma_{\tau}^{\prime}} \leq \frac{C D}{\tau^{\frac{3}{2}-\delta}} \quad\left(\sqrt[n-2]{2 m} \leq r<R, \tau>\tau_{0}\right)
$$

where $\Sigma_{\tau}^{\prime}$ and $R$ are as in Theorem 1.

\footnotetext{
${ }^{2}$ The "redshift" proposition and the "integrated local energy decay" proposition are to be found on page 526 in Section 3 and page 532 in Section 4 respectively.

${ }^{3}$ In this paper we use the term "interior" to refer to a region of finite radius; i.e., the term "interior region" is used interchangeably with "a region of compact $r$ (including the horizon)", and is of course not meant to refer to the interior of the black hole, which is not considered in this paper.
} 
Remark (decay rates and method of proof). Theorems 1 and 2 extend the presently known decay results for linear waves on $(3+1)$-dimensional Schwarzschild black holes to higher dimensions $n>3$; for $(3+1)$-dimensional Schwarzschild black holes, (1-9) was first established in [Dafermos and Rodnianski 2009b], and (1-10), (1-11) more recently in [Luk 2010]. However, both proofs use multipliers with weights in $t$, [Dafermos and Rodnianski 2009b] by using the conformal Morawetz vector field in the decay argument, and [Luk 2010] by using in addition the scaling vector field. Here we extend (1-9) to higher dimensions $n>3$ in the spirit of [Dafermos and Rodnianski 2010] only using multipliers with weights in $r$, and provide a new proof of the improved decay results (1-10) and (1-11) in the $n=3$-dimensional case in particular.

1B. Overview of the proof. In this section we give an overview of the work in this paper and present some of the ideas in the proof that lead to Theorem 1; references to previous work are made when useful, but for a more detailed account of previous work on the wave equation on Schwarzschild black hole spacetimes see Section 1.3 in [Dafermos and Rodnianski 2011] and references therein.

Energy identities. Let us recall that the wave equation (1-1) arises from an action principle and that the corresponding energy momentum tensor is conserved. Indeed, here we find (1-4) and by virtue of the wave equation (1-1)

$$
\nabla^{\mu} T_{\mu \nu}=\left(\square_{g} \phi\right)\left(\partial_{\nu} \phi\right)=0 .
$$

Moreover, the energy momentum tensor (1-4) satisfies the positivity condition, namely $T(X, Y) \geq 0$ for all future-directed causal vectors $X, Y$ at a point.

Now let $X$ be a vector field on $\mathcal{M}$. We define the energy current $J^{X}[\phi]$ associated to the multiplier $X$ by

$$
J_{\mu}^{X}[\phi] \doteq T_{\mu \nu}[\phi] X^{\nu} .
$$

Then

$$
K^{X} \doteq \nabla^{\mu} J_{\mu}^{X}={ }^{(X)} \pi^{\mu \nu} T_{\mu \nu}
$$

where we have used that $T_{\mu \nu}$ is conserved and symmetric. Here

$$
{ }^{(X)} \pi(Y, Z) \doteq \frac{1}{2}\left(\mathcal{L}_{X} g\right)(Y, Z)=\frac{1}{2} g\left(\nabla_{Y} X, Z\right)+\frac{1}{2} g\left(Y, \nabla_{Z} X\right)
$$

is the deformation tensor of $X$.

Remark. If $X$ is a Killing field, i.e., $X$ generates a 1-parameter group of isometries of $g,{ }^{(X)} \pi=0$, then $K^{X}=0$; i.e., $J^{X}$ is conserved.

In the following we shall refer to

$$
\int_{\mathcal{R}} K^{X} \mathrm{~d} \mu_{g}=\int_{\partial \mathcal{R}}^{*} J^{X}
$$

as the energy identity for $J^{X}$ (or simply $X$ ) on $\mathcal{R}$, where $\mathcal{R} \subset \mathcal{M}$ (this is of course the content of Stokes' theorem, and ${ }^{*} J$ denotes the Hodge-dual of $J$; see also Appendix B). Moreover we refer to $X$ in (1-16) as the multiplier vector field. In this paper we will largely be concerned with the construction of vector 
fields $X$, associated currents $J^{X}$ and their modifications, and the application of (1-16) and various derived energy inequalities to appropriately chosen domains $\mathcal{R} \subset \mathcal{D}$.

The new approach [Dafermos and Rodnianski 2010] to obtaining robust decay estimates requires us to first establish (i) uniform boundedness of energy, (ii) an integrated local energy decay estimate and (iii) good asymptotics towards null infinity.

Redshift effect. The reason (i) is nontrivial as compared to Minkowski space is that the energy corresponding to the multiplier $\partial_{t}$ degenerates on the horizon (the vector field $\partial_{t}$ becomes null on the horizon and no control on the angular derivatives is obtained; cf. [ibid. 2008]); it was recognized in [ibid. 2009b], and formulated more generally in [ibid. 2008], that the redshift property of Killing horizons is the key to obtaining an estimate for the nondegenerate energy (i.e., an energy with respect to a strictly timelike vector field up to the horizon, which controls all derivatives tangential to the horizons). An explicit construction of a suitable timelike vector field $N$ is given in Section 3 which allows us to state the redshift property in the language of multipliers and energy currents, and a proof of the uniform boundedness of the nondegenerate energy is given (independently of other calculations in this work) in Section 5A.

Integrated local energy decay. Section 4 is devoted to establishing (ii). This is achieved by the use of radial multiplier vector fields of the form $f\left(r^{*}\right) \partial_{r^{*}}$ (see Section 4A). In Section 4B a construction of a positive definite current for the high angular frequency regime is given using a decomposition on the sphere. In Section 4C a more general construction of a current is given using a commutation with the angular momentum operators. We wish to emphasize that the decay results of Section 5-albeit with a higher loss of differentiability - could be obtained solely on the basis of the latter current, without the recourse in Section 4B to the Fourier expansion on the sphere. However, the dependence on the initial data is significantly improved by virtue of the integrated local energy decay estimate Proposition 4.1; here (see Section 4D.1) the results of Sections 4B and 4C are combined in order to replace the commutation with the angular momentum operators by a commutation with the vector field $\partial_{t}$ only. The difficulty in both constructions lies in overcoming the "trapping" obstruction, which is the insight that it is impossible to prove an integrated local energy decay estimate on spacetime regions that contain the photon sphere without losing derivatives (see [Dafermos and Rodnianski 2008]). In the context of the Schwarzschild spacetime the need for vector fields whose associated currents give rise to positive definite spacetime integrals was first recognized and used in [Blue and Soffer 2003; Dafermos and Rodnianski 2009b], and such estimates have since then been extended by many authors [Marzuola et al. 2010; Alinhac 2009].

The p-hierarchy. In Section 5B we use a multiplier of the form $r^{p} \partial_{v^{*}}$ that gives rise to a weighted energy inequality which we consequently exploit in a hierarchy of two steps; this approach - which yields the corresponding quadratic decay rate in (1-9) — was pioneered in [Dafermos and Rodnianski 2010] for a large class of spacetimes, including the $(3+1)$-dimensional Schwarzschild and Kerr black hole spacetimes. In Section 5C a further commutation with $\partial_{v^{*}}$ is carried out, which allows us to extend the hierarchy of commuted weighted energy inequalities to four steps, yielding the corresponding decay rate for the first-order energy. The argument involves dealing with an (arbitrarily small) degeneracy of the 
first-order energy density at infinity which corresponds to the $\delta$-loss in the decay estimate (1-10). In both cases (iii) is ensured by the imposition of higher-order finite energy conditions on the initial data.

Interpolation. The pointwise decay of Theorem 2 then follows from Theorem 1 and the (refined) integrated local energy decay estimates of Section 4D.2 by a simple interpolation argument given in Section 6.

Final comments. The currents in Sections $4 \mathrm{~B}$ and $4 \mathrm{C}$ and the corresponding integrated local energy decay result already appeared in [Schlue 2010]. Independently a version of integrated local energy decay was subsequently obtained in [Laul and Metcalfe 2012]. In [Schlue 2010] there is also an alternative proof of (1-9) of Theorem 1 using the conformal Morawetz vector field.

\section{Global causal geometry of the higher-dimensional Schwarzschild solution}

In this section, we give a discussion (in the spirit of Section 3 of [Christodoulou 1995]) of the global geometry of the $(n+1)$-dimensional Schwarzschild black hole spacetime [Tangherlini 1963], the underlying manifold on which the wave equation is studied in this paper.

The $(n+1)$-dimensional Schwarzschild spacetime manifold $\mathcal{M} \doteq \mathcal{M}_{m}^{n}(n \geq 3, n \in \mathbb{N}, m>0)$ is spherically symmetric; i.e., $\mathrm{SO}(n)$ acts by isometry. The group orbits are $(n-1)$-spheres, and the quotient $\mathcal{Q}=\mathcal{M} / \mathrm{SO}(n)$ is a 2-dimensional Lorentzian manifold. The metric $g$ on $\mathcal{M}$ assumes the form

$$
g=\stackrel{\mathcal{O}}{g}+\gamma_{r}=\stackrel{\mathcal{Q}}{g}+r^{2} \stackrel{\circ}{n-1}
$$

where $\stackrel{\mathcal{Q}}{g}$ is the Lorentzian metric on $\mathcal{Q}$ to be discussed below, $\stackrel{\circ}{n-1}_{n-1}$ is the standard metric on $\mathbb{S}^{n-1}$, and $r$ is the area radius (the area of the $(n-1)$-sphere at $x \in \mathcal{Q}$ is given by $\omega_{n} r^{n-1}(x)$, where $\omega_{n}=2 \pi^{\frac{n}{2}} / \Gamma\left(\frac{n}{2}\right)$ is the area of the unit $(n-1)$-sphere); or more precisely, in local coordinates $x^{a}: a=1,2$ on $\mathcal{Q}$, and local coordinates $y^{A}: A=1, \ldots, n-1$ on $\mathbb{S}^{n-1}$,

$$
g_{(x, y)}=g_{a b}(x) \mathrm{d} x^{a} \mathrm{~d} x^{b}+r^{2}(x)\left(\stackrel{\circ}{\gamma-1}_{A B} \mathrm{~d} y^{A} \mathrm{~d} y^{B} .\right.
$$

The Schwarzschild spacetime is a solution of the vacuum Einstein equations, which in other words means that its Ricci curvature vanishes identically. This implies in particular (see derivation in [Schlue 2012]) that the area radius function $r$ satisfies the Hessian equations

$$
\nabla_{a} \partial_{b} r=\frac{(n-2)}{2 r}\left[1-\left(\partial^{c} r\right)\left(\partial_{c} r\right)\right] g_{a b},
$$

as a result of which the mass function $m$ on $\mathcal{Q}$ defined $^{4}$ by

$$
1-\frac{2 m}{r^{n-2}}=g^{a b} \partial_{a} r \partial_{b} r
$$

is constant; see [ibid.]; we take this parameter $m$ to be positive.

On $\mathcal{Q}$ we choose functions $u, v$ whose level sets are outgoing and incoming null curves, respectively, which are increasing towards the future. These functions define a null system of coordinates, in which the

\footnotetext{
${ }^{4}$ We choose the normalization of the mass function to be independent of the dimension $n$; this is motivated by a consideration of the mass equations in the presence of matter; see [Schlue 2012].
} 
metric $\stackrel{\mathcal{Q}}{g}$ takes the form

$$
\stackrel{\mathcal{Q}}{g}=-\Omega^{2} \mathrm{~d} u \mathrm{~d} v
$$

The Hessian equations (2-2) in null coordinates read

$$
\begin{aligned}
\frac{\partial^{2} r}{\partial u^{2}}-\frac{2}{\Omega} \frac{\partial \Omega}{\partial u} \frac{\partial r}{\partial u} & =0, \\
\frac{\partial^{2} r}{\partial u \partial v}+\frac{n-2}{r} \frac{\partial r}{\partial u} \frac{\partial r}{\partial v} & =-\frac{n-2}{4 r} \Omega^{2}, \\
\frac{\partial^{2} r}{\partial v^{2}}-\frac{2}{\Omega} \frac{\partial \Omega}{\partial v} \frac{\partial r}{\partial v} & =0,
\end{aligned}
$$

and the defining equation for the mass function (2-3) is

$$
1-\frac{2 m}{r^{n-2}}=-\frac{4}{\Omega^{2}} \frac{\partial r}{\partial u} \frac{\partial r}{\partial v}
$$

The system (2-5b), (2-6) can be rewritten as the partial differential equation

$$
\frac{\partial r^{*}}{\partial u \partial v}=0
$$

for a new radial function $r^{*}(r)$ that is related to $r$ by

$$
\frac{\mathrm{d} r^{*}}{\mathrm{~d} r}=\frac{1}{1-\frac{2 m}{r^{n-2}}} .
$$

A solution of (2-7), (2-8) is given by ${ }^{5}$

$$
r^{*}=\frac{1}{(n-2)} \sqrt[n-2]{2 m} \log |u v|
$$

or

$$
|u v|=e^{\frac{(n-2) r^{*}}{n-2} \sqrt{2 m}}=e^{\frac{(n-2) r}{n-2} \sqrt{2 m}} \exp \left[\left.\int \frac{n-2}{x^{n-2}-1} \mathrm{~d} x\right|_{x=\frac{r}{n-2 \sqrt{2 m}}}\right] .
$$

We find more explicitly, by an elementary integration (see [Schlue 2012]), that

$$
u v= \begin{cases}e^{\frac{r}{2 m}}\left(1-\frac{r}{2 m}\right), & n=3, \\ e^{\frac{2 r}{\sqrt{2 m}}} \frac{\left(1-\frac{r}{\sqrt{2 m}}\right)}{\left(1+\frac{r}{\sqrt{2 m}}\right)}, & n=4,\end{cases}
$$

\footnotetext{
${ }^{5}$ Here the representation in terms of null coordinates is such that $r^{*}=-\infty$ is contained in the $(u, v)$ plane and the metric is nondegenerate at $r=\sqrt[n-2]{2 m}$.
} 
and

$$
\begin{aligned}
& u v=e^{\frac{(n-2) r}{n-2} \sqrt{2 m}}\left(1-\frac{r}{\sqrt[n-2]{2 m}}\right) \begin{cases}1, & n \text { odd }, \\
\left(1+\frac{r}{\sqrt[n-2]{2 m}}\right)^{-1}, & n \text { even },\end{cases} \\
& \times \prod_{j=1}^{\left[\frac{n-3}{2}\right]}\left(\frac{r^{2}}{(2 m)^{\frac{2}{n-2}}}-2 \cos \left(\frac{2 \pi j}{n-2}\right) \frac{r}{(2 m)^{\frac{1}{n-2}}}+1\right)^{\cos \left(2 \pi j \frac{n-3}{n-2}\right)}
\end{aligned}
$$

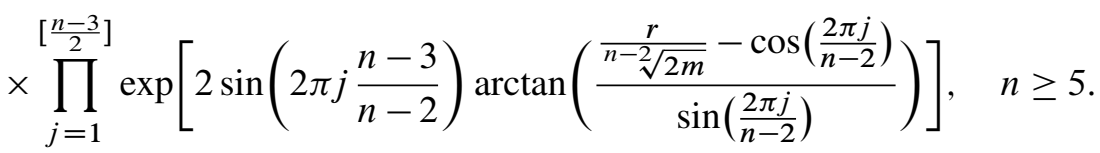

Note in particular that the $u=0$ and $v=0$ lines are the constant $r=\sqrt[n-2]{2 m}$ curves, and that all other curves of constant radius are hyperbolas in the $(u, v)$ plane - timelike for $r>\sqrt[n-2]{2 m}$, spacelike for $r<\sqrt[n-2]{2 m}$. This outlines the well-known global causal geometry of the Schwarzschild solution (see Figure 2).

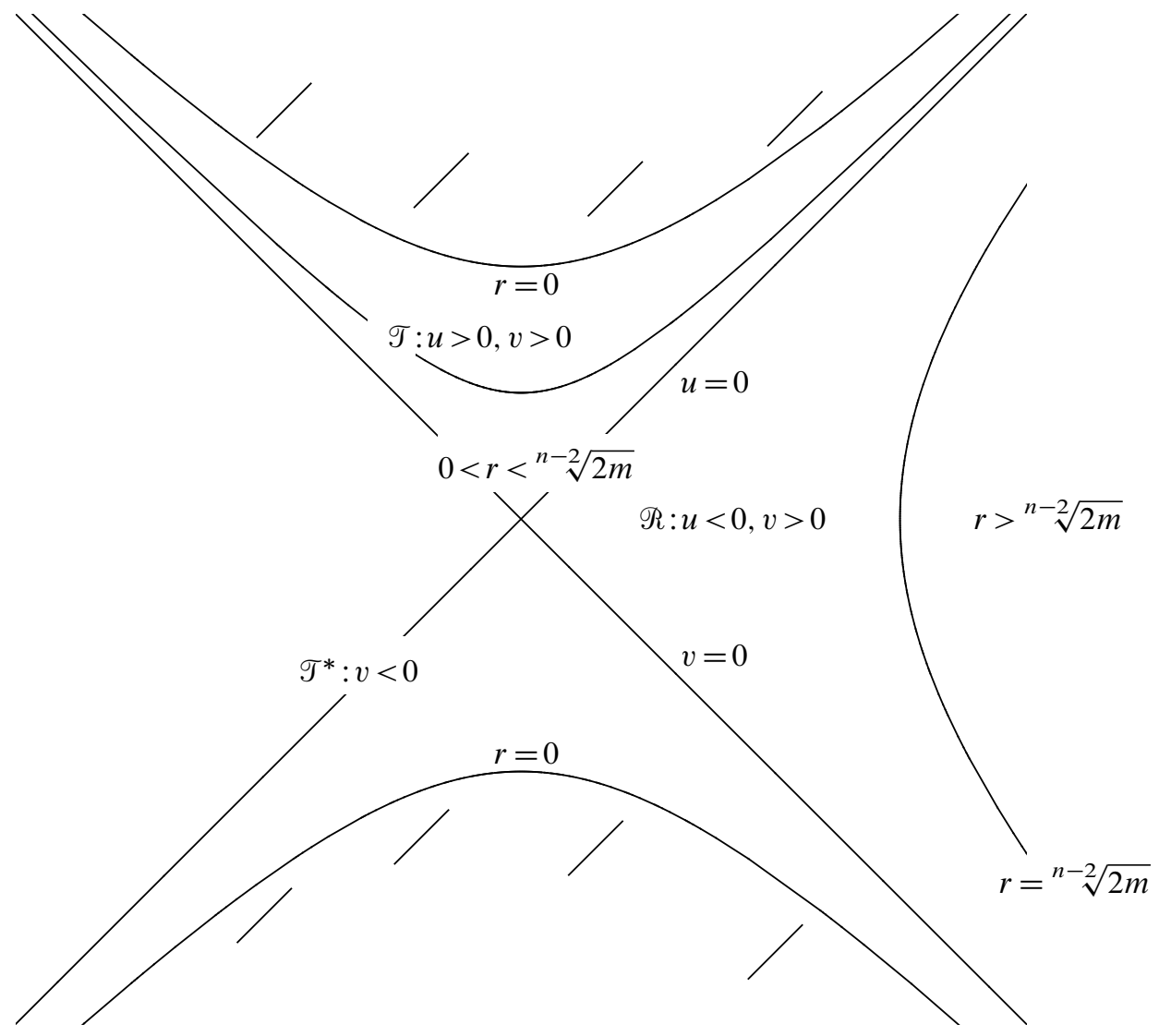

Figure 2. Global causal geometry of the Schwarzschild solution. 
It is easy to see [Schlue 2012] that for (2-9) the trapped region, the apparent horizon, the exterior, and the antitrapped regions, respectively, are given by

$$
\begin{aligned}
& \mathscr{T} \doteq\left\{(u, v) \in \mathcal{Q}: \frac{\partial r}{\partial u}<0, \frac{\partial r}{\partial v}<0\right\}=\{(u, v) \in \mathcal{Q}: u>0, v>0\}, \\
& \mathscr{A} \doteq\left\{(u, v) \in \mathcal{Q}: \frac{\partial r}{\partial u}<0, \frac{\partial r}{\partial v}=0\right\}=\{(u, v) \in \mathcal{Q}: u=0, v>0\}, \\
& \mathscr{R} \doteq\left\{(u, v) \in \mathcal{Q}: \frac{\partial r}{\partial u}<0, \frac{\partial r}{\partial v}>0\right\}=\{(u, v) \in \mathcal{Q}: u<0, v>0\}, \\
& \mathscr{T}^{*} \doteq\left\{(u, v) \in \mathcal{Q}: \frac{\partial r}{\partial u}>0\right\}=\{(u, v) \in \mathcal{Q}: v<0\} .
\end{aligned}
$$

Note this forms a partition of $\mathcal{Q}=\overline{\mathscr{T} \cup \mathscr{A} \cup \mathscr{R} \cup \mathscr{T}^{*}}$, and that in view of (2-6), $r<\sqrt[n-2]{2 m}$ in $\mathscr{T}$, $r=\sqrt[n-2]{2 m}$ in $\mathscr{A}$ and $r>\sqrt[n-2]{2 m}$ in $\mathscr{R}$. We shall refer to

$$
\mathcal{D} \doteq \overline{\mathcal{R}}=\{(u, v) \in \mathcal{Q}: u \leq 0, v \geq 0\}
$$

as the domain of outer communications.

Finally,

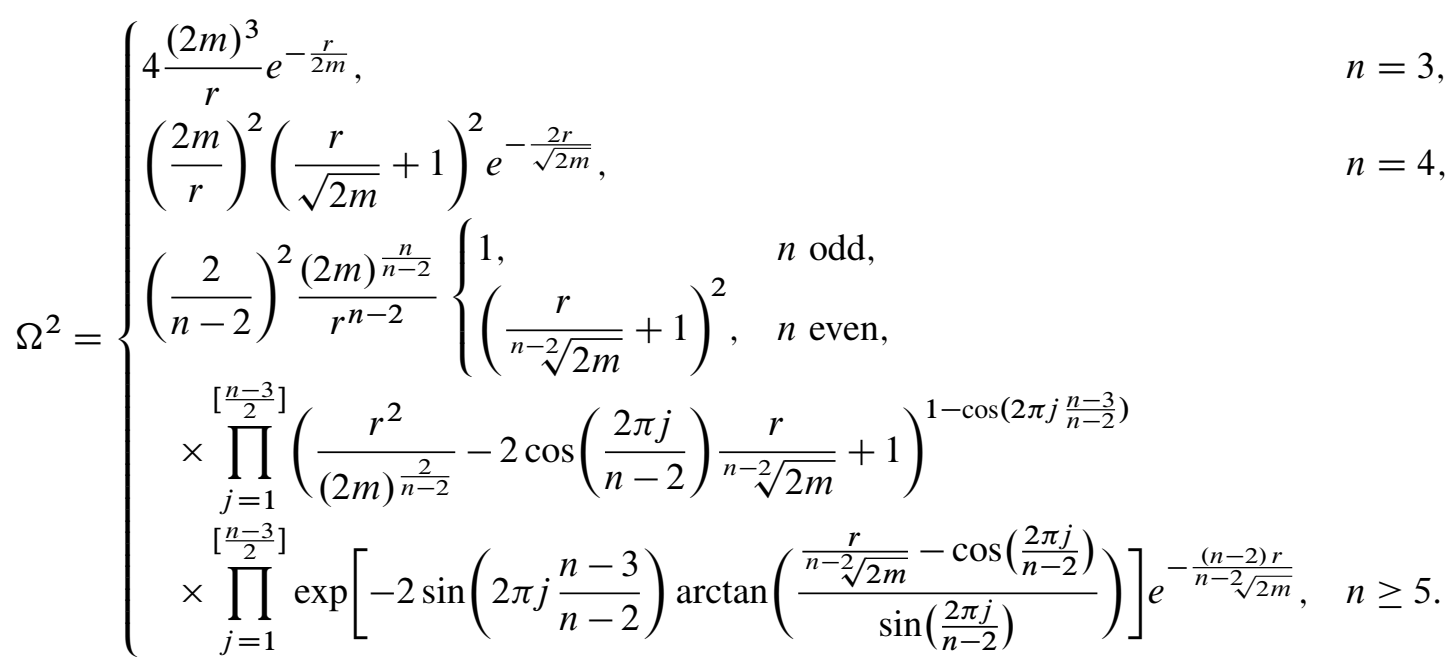

One may now also think of $r$ as a function of $u, v$ implicitly defined by (2-10) and (2-11). In $\mathscr{R}$ where $r>\sqrt[n-2]{2 m}$ (and $v-u>|u+v|), r$ may be complemented by

$$
t=\frac{2}{n-2} \sqrt[n-2]{2 m} \operatorname{arctanh}\left(\frac{u+v}{v-u}\right)
$$

note

$$
\mathrm{d} t=\frac{1}{n-2} \sqrt[n-2]{2 m}\left(\frac{1}{v} \mathrm{~d} v-\frac{1}{u} \mathrm{~d} u\right)
$$

and we will denote by $\bar{\Sigma}_{t}$ the corresponding level sets in $\mathcal{D}$. 
We find in these coordinates the classic expression for the Schwarzschild metric in the exterior region:

$$
g=-\left(1-\frac{2 m}{r^{n-2}}\right) \mathrm{d} t^{2}+\left(1-\frac{2 m}{r^{n-2}}\right)^{-1} \mathrm{~d} r^{2}+r^{2} \stackrel{\circ}{n-1} .
$$

In Regge-Wheeler coordinates $\left(t, r^{*}\right)$, where $r^{*}$ is centered at the photon sphere $r=\sqrt[n-2]{n m}$ :

$$
r^{*}=\int_{(n m)^{\frac{1}{n-2}}}^{r} \frac{1}{1-\frac{2 m}{r^{\prime n-2}}} \mathrm{~d} r^{\prime}
$$

the metric obviously takes the conformally flat form

$$
g=\left(1-\frac{2 m}{r^{n-2}}\right)\left(-\mathrm{d} t^{2}+\mathrm{d} r^{* 2}\right)+r^{2} \stackrel{\gamma}{n-1} .
$$

We shall also use the Eddington-Finkelstein coordinates

$$
u^{*}=\frac{1}{2}\left(t-r^{*}\right), \quad v^{*}=\frac{1}{2}\left(t+r^{*}\right),
$$

which are again double null coordinates:

$$
g=-4\left(1-\frac{2 m}{r^{n-2}}\right) \mathrm{d} u^{*} \mathrm{~d} v^{*}+r^{2} \dot{\gamma}_{n-1}
$$

The two systems of null coordinates in $\mathscr{R}$ are related by

$$
u=-e^{-\frac{(n-2) u^{*}}{n-2 \sqrt{2 m}}}, \quad v=e^{\frac{(n-2) v^{*}}{n-2 m}}
$$

\section{The redshift effect}

In this section we prove a manifestation of the local redshift effect in the Schwarzschild geometry of Section 2 in the framework of multiplier vector fields.

Proposition 3.1 (local redshift effect). Let $\phi$ be a solution of the wave equation (1-1). Then there exists a $\varphi_{t}$-invariant future-directed timelike smooth vector field $N$ on $\mathcal{D}$, two radii $\sqrt[n-2]{2 m}<r_{0}^{(N)}<r_{1}^{(N)}$, and a constant $b>0$ such that

$$
K^{N}(\phi) \geq b\left(J^{N}(\phi), N\right) \quad\left(\sqrt[n-2]{2 m} \leq r<r_{0}^{(N)}\right)
$$

and $N=T\left(r \geq r_{1}^{(N)}\right)$.

The vector field $N$ will be constructed explicitly with the following vector fields.

$T$-vector field. Here $\varphi_{t}$ is the 1-parameter group of diffeomorphisms generated by the vector field

$$
T=\frac{1}{2} \frac{n-2}{\sqrt[n-2]{2 m}}\left(v \frac{\partial}{\partial v}-u \frac{\partial}{\partial u}\right)
$$

note that in $\mathscr{R}$, where $r>\sqrt[n-2]{2 m}$ (recall (2-15)),

$$
T=\frac{\partial}{\partial t} .
$$


$T$ is a Killing vector field:

$$
{ }^{(T)} \pi=0,
$$

which is timelike in the exterior, spacelike in the interior of the black hole and null on the horizon:

$$
g(T, T)=\frac{1}{4} \frac{(n-2)^{2}}{(2 m)^{\frac{2}{n-2}}} u v \Omega^{2}=-\left(1-\frac{2 m}{r^{n-2}}\right) \begin{cases}<0, & r>\sqrt[n-2]{2 m} \\ =0, & r=\sqrt[n-2]{2 m} \\ >0, & r<\sqrt[n-2]{2 m}\end{cases}
$$

In particular,

$$
\left.T\right|_{\mathcal{H}^{+}}=\frac{1}{2} \frac{n-2}{\sqrt[n-2]{2 m}} v \frac{\partial}{\partial v},\left.\quad T\right|_{\mathcal{H}^{+} \cap \mathcal{H}^{-}}=0
$$

$Y$-vector field. Let us also define a vector field $Y$ on $\mathcal{H}^{+}$conjugate to $T$ :

$$
\left.Y\right|_{\mathcal{H}^{+}}=-\frac{2}{\frac{\partial r}{\partial u}} \frac{\partial}{\partial u}
$$

Indeed,

$$
\left.g(T, Y)\right|_{\mathcal{H}^{+}}=-2
$$

because

$$
\left.\Omega^{2}\right|_{\mathcal{H}^{+}}=-4 \frac{\sqrt[n-2]{2 m}}{n-2} \frac{1}{v} \frac{\partial r}{\partial u} .
$$

Furthermore, as a consequence of $(2-5 b)$,

$$
\left.\frac{\partial^{2} r}{\partial u \partial v}\right|_{\mathcal{H}^{+}}=-\left.\frac{n-2}{4 r} \Omega^{2}\right|_{\mathcal{H}^{+}}=\left.\frac{1}{v} \frac{\partial r}{\partial u}\right|_{\mathcal{H}^{+}},
$$

and we have

$$
\left.[T, Y]\right|_{\mathcal{H}^{+}}=\left.[T, Y]^{u} \frac{\partial}{\partial u}\right|_{\mathcal{H}^{+}}+\left.[T, Y]^{v} \frac{\partial}{\partial v}\right|_{\mathcal{H}^{+}}=\left.\frac{n-2}{\sqrt[n-2]{2 m}} \frac{1}{\frac{\partial r}{\partial u}}\left[v \frac{1}{\frac{\partial r}{\partial u}} \frac{\partial^{2} r}{\partial u \partial v}-1\right] \frac{\partial}{\partial u}\right|_{\mathcal{H}^{+}}=0 .
$$

$E_{A}$-vector fields. We denote by $E_{A}: A=1, \ldots, n-1$ an orthonormal frame field tangential to the orbits of the spherical isometry:

$$
\begin{gathered}
g\left(E_{A}, E_{B}\right)=\delta_{A B}= \begin{cases}1, & A=B, \\
0, & A \neq B,\end{cases} \\
\left.g\left(E_{A}, Y\right)\right|_{\mathcal{H}^{+}}=0, \quad g\left(E_{A}, T\right)=\left.0\right|_{\mathcal{H}^{+}} \quad(A=1, \ldots, n-1) .
\end{gathered}
$$

We can now state that the surface gravity of the event horizon is positive; this is essential for the existence of the redshift effect (see more generally [Dafermos and Rodnianski 2008] and also [Aretakis 2011] for work where this is not the case).

Lemma 3.2 (surface gravity). On $\mathcal{H}^{+}$,

$$
\nabla_{T} T=\kappa_{n} T
$$


with

$$
\kappa_{n}=\frac{1}{2} \frac{n-2}{\sqrt[n-2]{2 m}}>0 .
$$

We call $\kappa_{n}$ the surface gravity.

Note. $T=\kappa_{n}\left(v \frac{\partial}{\partial v}-u \frac{\partial}{\partial u}\right)$.

Alternatively, $\kappa_{n}$ is characterized by

$$
\nabla_{T} Y=-\kappa_{n} Y
$$

on $\mathcal{H}^{+}$. Clearly

$$
g\left(\nabla_{T} Y, Y\right)=\frac{1}{2} T \cdot g(Y, Y)=0
$$

since $Y$ is null along $\mathcal{H}^{+}$, and

$$
g\left(\nabla_{T} Y, T\right) \stackrel{(3-8)}{=} g\left(\nabla_{Y} T, T\right) \stackrel{(3-3)}{=}-g\left(\nabla_{T} T, Y\right)=2 \kappa_{n} ;
$$

also

$$
g\left(\nabla_{T} Y, E_{A}\right) \stackrel{(3-8)}{=} g\left(\nabla_{Y} T, E_{A}\right) \stackrel{(3-3)}{=}-g\left(\nabla_{E_{A}} T, Y\right)=0 \quad \text { for } A=1, \ldots, n-1,
$$

because $\nabla_{E_{A}} T=0$. Note, for later use, on $\mathcal{H}^{+}$,

$$
\nabla_{E_{A}} Y=-\frac{2}{\sqrt[n-2]{2 m}} E_{A} .
$$

We defined $Y$ on $\mathcal{H}^{+}$conjugate to $T,\left.g(T, Y)\right|_{\mathcal{H}^{+}}=-2$. Next we extend $Y$ to a neighborhood of the horizon by

$$
\nabla_{Y} Y=-\sigma(Y+T) \quad\left(\sigma>\frac{16}{n-2}(2 m)^{\frac{3}{n-2}}\right)
$$

and then we extend $Y$ to $\mathscr{R}$ by Lie-transport along the integral curves of $T$ :

$$
[T, Y]=0 .
$$

Proposition 3.3 (redshift). For the future-directed timelike vector field

$$
N=T+Y
$$

there is a $b>0$ such that on $\mathcal{H}^{+}$

$$
K^{N} \geq b\left(J^{N}, N\right)
$$

Proof. Let us calculate

$$
\begin{aligned}
K^{Y}= & { }^{(Y)} \pi^{\mu \nu} T_{\mu \nu} \\
= & \frac{1}{4}\left\{{ }^{(Y)} \pi(T, T) T(Y, Y)+2^{(Y)} \pi(T, Y) T(Y, T)+{ }^{(Y)} \pi(Y, Y) T(T, T)\right\} \\
& \quad-\sum_{A=1}^{n-1}\left\{(Y) \pi\left(E_{A}, Y\right) T\left(E_{A}, T\right)+{ }^{(Y)} \pi\left(E_{A}, T\right) T\left(E_{A}, Y\right)\right\}+\sum_{A, B=1}^{n-1}(Y) \pi\left(E_{A}, E_{B}\right) T\left(E_{A}, E_{B}\right) .
\end{aligned}
$$


Now, on one hand, on $\mathcal{H}^{+}$,

$$
\begin{gathered}
(Y) \pi(T, T)=2 \kappa_{n}, \quad(Y) \pi(T, Y)=\sigma, \quad(Y) \pi(Y, Y)=2 \sigma \\
{ }^{(Y)} \pi\left(E_{A}, Y\right)=0, \quad{ }^{(Y)} \pi\left(E_{A}, T\right)=0, \quad(Y) \pi\left(E_{A}, E_{B}\right)=-\frac{2}{\sqrt[n-2]{2 m}} \delta_{A B} .
\end{gathered}
$$

Thus

$$
K^{Y}=\frac{1}{2} \kappa_{n} T(Y, Y)+\frac{1}{2} \sigma T(Y+T, T)-\frac{2}{\sqrt[n-2]{2 m}} \sum_{A=1}^{n-1} T\left(E_{A}, E_{A}\right)
$$

On the other hand, on $\mathcal{H}^{+}$,

$$
T(Y, Y)=\left(\frac{2}{\frac{\partial r}{\partial u}} \frac{\partial \phi}{\partial u}\right)^{2}, \quad T(Y, T)=|\not \nabla \phi|_{r^{2} \dot{\gamma}_{n-1}}^{2}, \quad T(T, T)=\left(\kappa_{n} v \frac{\partial \phi}{\partial v}\right)^{2}
$$

and, on $\mathcal{H}^{+}$,

$$
T\left(E_{A}, E_{B}\right)=\left(E_{A} \cdot \phi\right)\left(E_{B} \cdot \phi\right)-\frac{1}{2}(2 m)^{\frac{2}{n-2}} \delta_{A B}|\not \nabla \phi|_{r^{2} \gamma_{n-1}}-\frac{1}{2}(n-2)(2 m)^{\frac{1}{n-2}} \frac{v}{\frac{\partial r}{\partial u}} \delta_{A B}\left(\frac{\partial \phi}{\partial u}\right)\left(\frac{\partial \phi}{\partial v}\right)
$$

Using Cauchy's inequality, on $\mathcal{H}^{+}$,

$$
\begin{aligned}
& -\frac{2}{\sqrt[n-2]{2 m}} \sum_{A=1}^{n-1} T\left(E_{A}, E_{A}\right)=(n-3)(2 m)^{\frac{1}{n-2}}|\not \nabla \phi|_{r^{2} \dot{\gamma}_{n-1}}^{2}+(n-2)(n-1) \frac{v}{\frac{\partial r}{\partial u}}\left(\frac{\partial \phi}{\partial u}\right)\left(\frac{\partial \phi}{\partial v}\right) \\
& \geq(n-3)(2 m)^{\frac{1}{n-2}} T(Y, T)-\frac{1}{4} \kappa_{n} T(Y, Y)-\frac{1}{\kappa_{n}} \frac{2(n-1)}{(n-2)}(2 m)^{\frac{2}{n-2}} T(T, T) \\
& \geq-\frac{1}{4} \kappa_{n} T(Y, Y)-\frac{n-1}{\kappa_{n}^{2}}(2 m)^{\frac{1}{n-2}} T(T, T) .
\end{aligned}
$$

Since we have chosen $\sigma>2 \frac{n-1}{\kappa_{n}^{2}}(2 m)^{\frac{1}{n-2}}, K^{Y}$ has a sign,

$$
K^{Y} \geq \frac{1}{4} \kappa_{n} T(Y, Y)+\sigma^{\prime} T(Y+T, T)
$$

for $0<\sigma^{\prime}<\frac{\sigma}{2}-\frac{n-1}{\kappa_{n}^{2}}(2 m)^{\frac{1}{n-2}}$, or

$$
K^{Y} \geq b T(Y+T, Y+T)
$$

for $0<b<\min \left\{\frac{\kappa_{n}}{4}, \frac{\sigma^{\prime}}{2}\right\}$. This yields the result

$$
K^{N}=K^{Y} \geq b T(N, N)=b\left(J^{N}, N\right) .
$$

Finally, we find an explicit expression for $Y$. Consider the vector field

$$
\hat{Y}=-\frac{2}{\frac{\partial r}{\partial u}} \frac{\partial}{\partial u}
$$


on $\mathscr{R} \cup \mathscr{A}$ formally defined by the expression for $Y$ on $\mathcal{H}^{+}$. In $\mathscr{R}$

$$
\hat{Y}=\frac{2}{1-\frac{2 m}{r^{n-2}}} \frac{\partial}{\partial u^{*}} \text {. }
$$

$\hat{Y}$ generates geodesics, this being a consequence of the Hessian equations (2-5a),

$$
\nabla_{\hat{Y}} \hat{Y}=\left(\frac{2}{\frac{\partial r}{\partial u}}\right)^{2}\left[-\frac{1}{\frac{\partial r}{\partial u}} \frac{\partial^{2} r}{\partial u^{2}}+\frac{2}{\Omega} \frac{\partial \Omega}{\partial u}\right] \frac{\partial}{\partial u}=0,
$$

and is Lie-transported by $T$ :

$$
[T, \hat{Y}]=\frac{2}{\left(\frac{\partial r}{\partial u}\right)^{2}}\left(\left[T, \frac{\partial}{\partial u}\right] \cdot r\right) \frac{\partial}{\partial u}-\frac{2}{\frac{\partial r}{\partial u}}\left[T, \frac{\partial}{\partial u}\right]=-\kappa_{n} \hat{Y}+\kappa_{n} \hat{Y}=0
$$

because $\left[T, \frac{\partial}{\partial u}\right]=\kappa_{n} \frac{\partial}{\partial u} . Y$ as constructed above coincides with

$$
Y=\alpha(r) \hat{Y}+\beta(r) T
$$

where

$$
\alpha(r)=1+\frac{\sigma}{4 \kappa_{n}}\left(1-\frac{2 m}{r^{n-2}}\right), \quad \beta(r)=\frac{\sigma}{4 \kappa_{n}}\left(1-\frac{2 m}{r^{n-2}}\right) .
$$

Indeed, on $\mathcal{H}^{+}$,

$$
\left.Y\right|_{\mathcal{H}^{+}}=\left.\hat{Y}\right|_{\mathcal{H}^{+}}=-\left.\frac{2}{\frac{\partial r}{\partial u}} \frac{\partial}{\partial u}\right|_{\mathcal{H}^{+}}
$$

and

$$
\left.\nabla_{Y} Y\right|_{\mathcal{H}^{+}}=\left.\nabla_{\hat{Y}} Y\right|_{\mathcal{H}^{+}}=\left.(\hat{Y} \cdot \alpha) \hat{Y}\right|_{\mathcal{H}^{+}}+\left.\nabla_{\hat{Y}} \hat{Y}\right|_{\mathcal{H}^{+}}+\left.(\hat{Y} \cdot \beta) T\right|_{\mathcal{H}^{+}}=-\left.\sigma(Y+T)\right|_{\mathcal{H}^{+}}
$$

since

$$
\left.\hat{Y} \cdot \alpha\right|_{\mathcal{H}^{+}}=\left.\frac{\sigma}{4 \kappa_{n}}(n-2) \frac{2 m}{r^{n-1}} \hat{Y} \cdot r\right|_{\mathcal{H}^{+}}=-\sigma,\left.\quad \hat{Y} \cdot \beta\right|_{\mathcal{H}^{+}}=-\sigma
$$

and $Y$ remains Lie-transported by $T$ :

$$
[T, Y]=(T \cdot \alpha) \hat{Y}+(T \cdot \beta) T+\alpha[T, \hat{Y}]+\beta[T, T]=0
$$

since

$$
T \cdot \alpha=0=T \cdot \beta .
$$

Thus the vector field $Y$ is given explicitly by

$$
Y= \begin{cases}-\frac{2}{\frac{\partial r}{\partial u}} \frac{\partial}{\partial u} & \text { on } \mathcal{H}^{+}, \\ {\left[1+\frac{\sigma}{4 \kappa_{n}}\left(1-\frac{2 m}{r^{n-2}}\right)\right] \frac{2}{1-\frac{2 m}{r^{n-2}} \frac{\partial}{\partial u^{*}}+\frac{\sigma}{4 \kappa_{n}}\left(1-\frac{2 m}{r^{n-2}}\right) \frac{\partial}{\partial t}}} & \text { in } \mathscr{R} .\end{cases}
$$


Clearly, by continuity, we can choose two values $\sqrt[n-2]{2 m}<r_{0}^{(N)}<r_{1}^{(N)}<\infty$ and set

$$
N=\left\{\begin{array}{lc}
T+Y, & \sqrt[n-2]{2 m} \leq r \leq r_{0}^{(N)} \\
T, & r \geq r_{1}^{(N)}
\end{array}\right.
$$

with a smooth $\varphi_{t}$-invariant transition of the timelike vector field $N$ in $r_{0}^{(N)} \leq r \leq r_{1}^{(N)}$, such that (3-17) extends to the neighborhood $\sqrt[n-2]{2 m}<r<r_{0}^{(N)}$ of the event horizon.

Remark 3.4. For a geometric interpretation of Proposition 3.3 see [Schlue 2012] and also [Dafermos and Rodnianski 2008].

\section{Integrated local energy decay}

In this section we prove several integrated local energy decay statements, i.e., estimates on the energy density of solutions to (1-1) integrated on (bounded) space-time regions; this in an essential ingredient for the decay mechanism employed in Section 5.

Let $\mathcal{R}_{r_{0}, r_{1}}\left(t_{0}, t_{1}, u_{1}^{*}, v_{1}^{*}\right)$ be the region composed of a trapezoid and characteristic rectangles as follows (see Figure 3):

$$
\begin{aligned}
\mathcal{R}_{r_{0}, r_{1}}\left(t_{0}, t_{1}, u_{1}^{*}, v_{1}^{*}\right) \doteq\left\{(t, r): t_{0} \leq t \leq t_{1}, r_{0} \leq r \leq r_{1}\right\} \\
\cup\left\{(t, r): r \leq r_{0}, \frac{1}{2}\left(t-r^{*}\right) \leq u_{1}^{*}, t_{0}+r_{0}^{*} \leq t+r^{*} \leq t_{1}+r_{0}^{*}\right\} \\
\cup\left\{(t, r): r \geq r_{1}, \frac{1}{2}\left(t+r^{*}\right) \leq v_{1}^{*}, t_{0}-r_{1}^{*} \leq t-r^{*} \leq t_{1}-r_{1}^{*}\right\} .
\end{aligned}
$$

We define

$$
\mathcal{R}_{r_{0}, r_{1}}^{\infty}\left(t_{0}\right) \doteq \bigcup_{t_{1} \geq t_{0}} \bigcup_{u_{1}^{*} \geq \frac{1}{2}\left(t_{1}-r_{0}^{*}\right)} \bigcup_{v_{1}^{*} \geq \frac{1}{2}\left(t_{1}+r_{1}^{*}\right)} \mathcal{R}\left(t_{0}, t_{1}, u_{1}^{*}, v_{1}^{*}\right)
$$

and denote its past boundary by

$$
\Sigma_{\tau_{0}} \doteq \partial^{-} \mathcal{R}_{r_{0}, r_{1}}^{\infty}\left(t_{0}\right), \quad \tau_{0}=\frac{1}{2}\left(t_{0}-r_{1}^{*}\right) .
$$

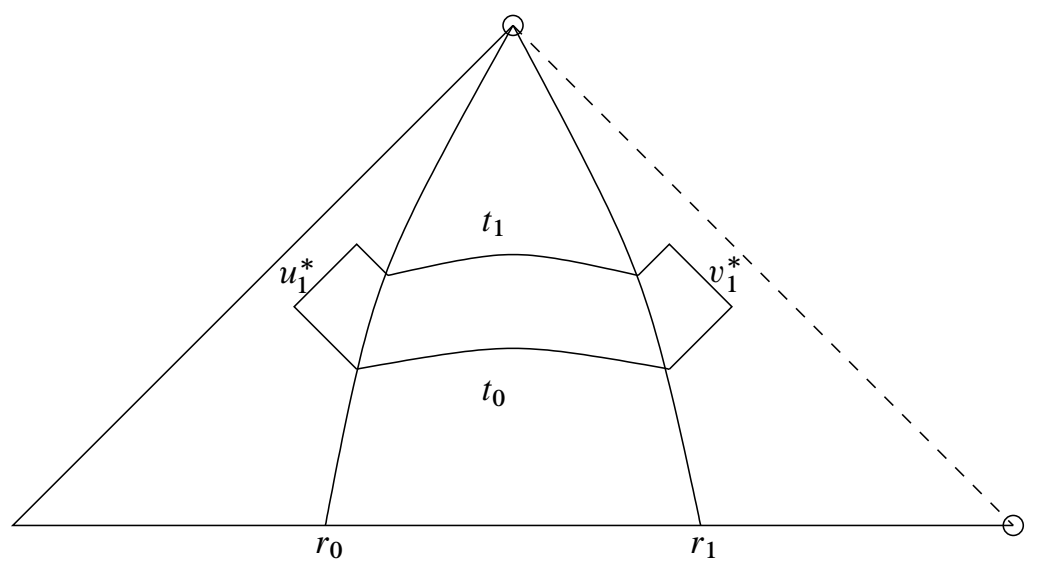

Figure 3. The region $\mathcal{R}_{r_{0}, r_{1}}\left(t_{0}, t_{1}, u_{1}^{*}, v_{1}^{*}\right)$. 
We shall first state the central estimate.

Proposition 4.1 (integrated local energy decay estimate). There exist $(2 m)^{\frac{1}{n-2}}<r_{0}<r_{1}<\infty$ and a constant $C(n, m)$ depending only on the dimension $n$ and the mass $m$, such that for any given solution $\phi$ of the wave equation $\square_{g} \phi=0$,

$$
\begin{aligned}
\int_{\mathcal{R}_{r_{0}, r_{1}}^{\infty}\left(t_{0}\right)}\left\{\frac{1}{r^{n}}\left(\frac{\partial \phi}{\partial r^{*}}\right)^{2}+\frac{1}{r^{n+1}}\left(\frac{\partial \phi}{\partial t}\right)^{2}+\frac{1}{r^{3}}\left(1-\frac{2 m}{r^{n-2}}\right)|\not \phi|_{r^{2} \dot{\gamma}_{n-1}}^{2}\right\} \mathrm{d} \mu_{g} & \leq C(n, m) \int_{\Sigma_{\tau_{0}}}\left(J^{T}(\phi)+J^{T}(T \cdot \phi), n\right)
\end{aligned}
$$

for all $t_{0} \geq 0$, where $\tau_{0}=\frac{1}{2}\left(t_{0}-r_{1}^{*}\right)$.

The degeneracy at infinity can in fact be improved:

Proposition 4.2 (improved integrated local energy decay estimate). Let $\phi$ be a solution of the wave equation $\square_{g} \phi=0$. Then there exists a constant $C(n, m, \delta)$ for each $0<\delta<1$ such that

$$
\begin{aligned}
\int_{\mathcal{R}_{r_{0}, r_{1}}^{\infty}\left(t_{0}\right)}\left\{\frac{1}{r^{1+\delta}}\left(\frac{\partial \phi}{\partial r^{*}}\right)^{2}+\frac{1}{r^{1+\delta}}\left(\frac{\partial \phi}{\partial t}\right)^{2}+\frac{1}{r}\left(1-\frac{2 m}{r^{n-2}}\right)|\not \phi|_{r^{2} \dot{\gamma}_{n-1}}^{2}\right\} \mathrm{d} \mu_{g} & \leq C(n, m, \delta) \int_{\Sigma_{\tau_{0}}}\left(J^{T}(\phi)+J^{T}(T \cdot \phi), n\right)
\end{aligned}
$$

for any $t_{0} \geq 0$, where $r_{0}<r_{1}$ are as above, and $\tau_{0}=\frac{1}{2}\left(t_{0}-r_{1}^{*}\right)$.

As a consequence of the redshift effect of Section 3 and the uniform boundedness of the nondegenerate energy (which is proven independently in Section 5A), we can infer in a more geometric formulation:

Corollary 4.3 (nondegenerate integrated local energy decay). Let $\phi$ be a solution of (1-1). Then for any $R>\sqrt[n-2]{2 m}$ there exists a constant $C(n, m, R)$ such that

$$
\int_{\tau^{\prime}}^{\tau} \mathrm{d} \bar{\tau} \int_{\Sigma_{\bar{\tau}}^{\prime}}\left(J^{N}(\phi), n\right) \leq C(n, m, R) \int_{\Sigma_{\tau^{\prime}}}\left(J^{N}(\phi)+J^{T}(T \cdot \phi), n\right),
$$

for all $\tau^{\prime}<\tau$, where $\Sigma_{\tau}^{\prime} \doteq \Sigma_{\tau} \cap\{r \leq R\}$.

Proof. Let

$$
\mathcal{R}^{\prime}\left(\tau^{\prime}, \tau\right) \doteq \mathrm{J}^{-}\left(\Sigma_{\tau}^{\prime}\right) \cap \mathrm{J}^{+}\left(\Sigma_{\tau^{\prime}}\right) .
$$

In $\mathcal{R}^{\prime}\left(\tau^{\prime}, \tau\right) \cap\left\{r<r_{0}^{(N)}\right\}$ we have by Proposition 3.1

$$
\left(J^{N}(\phi), n\right) \leq \frac{1}{b} K^{N}(\phi),
$$

and in $\mathcal{R}^{\prime}\left(\tau^{\prime}, \tau\right) \cap\left\{r \geq r_{1}^{(N)}\right\}$ trivially $\left(J^{N}(\phi), n\right) \leq\left(J^{T}(\phi), n\right)$. Therefore using the energy identity for $N$ on $\mathcal{R}^{\prime}\left(\tau^{\prime}, \tau\right)$ the estimate (4-6) follows from Proposition 5.2 and Proposition 4.1.

In the above, no control is obtained on a spacetime integral of $\phi^{2}$ itself; however, all that is needed for the decay argument of Section 5 is an estimate for the integral of $\phi^{2}$ on timelike boundaries. 
Proposition 4.4 (zeroth-order terms on timelike boundaries). Let $\phi$ be solution of the wave equation (1-1), and $R>\sqrt[n-2]{8 n m}$. Then there is a constant $C(n, m, R)$ such that, for all $\tau^{\prime}<\tau$,

$$
\begin{aligned}
& \left.\int_{2 \tau^{\prime}+R^{*}}^{2 \tau+R^{*}} \mathrm{~d} t \int_{\mathbb{S}^{n-1}} \mathrm{~d} \mu_{\dot{\gamma}_{n-1}} \phi^{2}\right|_{r=R} \\
& \quad \leq\left. C(n, m, R) \int_{2 \tau^{\prime}+R^{*}}^{2 \tau+R^{*}} \mathrm{~d} t \int_{\mathbb{S}^{n-1}} \mathrm{~d} \mu_{\dot{\gamma}_{n-1}}\left\{\left(\frac{\partial \phi}{\partial r^{*}}\right)^{2}+|\not \phi|^{2}\right\}\right|_{r=R}+C(n, m, R) \int_{\Sigma_{\tau^{\prime}}}\left(J^{T}(\phi), n\right) .
\end{aligned}
$$

The central result of Proposition 4.1 combines results for two different regimes, that of high angular frequencies and that of low angular frequencies. First we will use radial multiplier vector fields to construct positive definite currents to deal with the former regime, and then a more general current using a commutation with angular momentum operators for the latter.

Remark 4.5. The specific parametrization (4-3) has technical advantages, but $\Sigma_{\tau}$ can in principle be replaced by a foliation of strictly spacelike hypersurfaces terminating at future null infinity and crossing the event horizon to the future of the bifurcation sphere.

4A. Radial multiplier vector fields. A radial multiplier is a vector field of the form

$$
X=f\left(r^{*}\right) \frac{\partial}{\partial r^{*}} .
$$

We would like the associated current to be positive; however we find in general, as it is shown below:

$$
K^{X}=\frac{f^{\prime}}{1-\frac{2 m}{r^{n-2}}}\left(\frac{\partial \phi}{\partial r^{*}}\right)^{2}+\frac{f}{r}\left(1-\frac{n m}{r^{n-2}}\right)|\not \phi \phi|_{r^{2} \dot{\gamma}_{n-1}}^{2}-\frac{1}{2}\left[f^{\prime}+(n-1) \frac{f}{r}\left(1-\frac{2 m}{r^{n-2}}\right)\right] \partial^{\alpha} \phi \partial_{\alpha} \phi
$$

Note. The prefactor to the angular derivatives vanishes at the photon sphere at $r=\sqrt[n-2]{n m}$.

Calculation of the deformation tensor ${ }^{(X)} \pi$. It is convenient to work in Eddington-Finkelstein coordinates:

$$
X=\frac{1}{2} f\left(r^{*}\right) \frac{\partial}{\partial v^{*}}-\frac{1}{2} f\left(r^{*}\right) \frac{\partial}{\partial u^{*}} .
$$

We then obtain for the components of the deformation tensor:

$$
\begin{gathered}
{ }^{(X)} \pi_{u^{*} u^{*}}=\left(1-\frac{2 m}{r^{n-2}}\right) f^{\prime}, \quad(X) \pi_{v^{*} v^{*}}=\left(1-\frac{2 m}{r^{n-2}}\right) f^{\prime}, \\
{ }^{(X)} \pi_{u^{*} v^{*}}=-\left(1-\frac{2 m}{r^{n-2}}\right)\left(f^{\prime}+(n-2) \frac{2 m}{r^{n-1}} f\right), \\
{ }^{(X)} \pi_{a A}=0, \quad{ }^{(X)} \pi_{A B}=f r\left(1-\frac{2 m}{r^{n-2}}\right)\left(\stackrel{\circ}{ }_{n-1}\right)_{A B} .
\end{gathered}
$$

The formula (4-9) for $K^{X}$ is now obtained by writing out (see also Appendix B)

$$
K^{X}={ }^{(X)} \pi^{\alpha \beta} T_{\alpha \beta}
$$


and rearranging the terms so as to complete $\left(\frac{\partial \phi}{\partial u^{*}}\right)^{2}+\left(\frac{\partial \phi}{\partial v^{*}}\right)^{2}$ to $\left(\frac{\partial \phi}{\partial r^{*}}\right)^{2}$. This rearrangement is also related to the following modification of currents; for observe that, if $\square \phi=0$,

$$
\square\left(\phi^{2}\right)=2\left(\partial^{\alpha} \phi\right)\left(\partial_{\alpha} \phi\right) .
$$

First modified current. With the notation

$$
J_{\mu}^{X, 0}=T_{\mu \nu} X^{v}
$$

define the first modified current by

$$
J_{\mu}^{X, 1}=J_{\mu}^{X, 0}+\frac{1}{4}\left(f^{\prime}+(n-1) \frac{f}{r}\left(1-\frac{2 m}{r^{n-2}}\right)\right) \partial_{\mu}\left(\phi^{2}\right)-\frac{1}{4} \partial_{\mu}\left(f^{\prime}+(n-1) \frac{f}{r}\left(1-\frac{2 m}{r^{n-2}}\right)\right) \phi^{2} .
$$

Consequently the divergences are

$$
\begin{aligned}
K^{X, 0} & =\nabla^{\mu} J_{\mu}^{X, 0}=K^{X} \\
K^{X, 1} & =\nabla^{\mu} J_{\mu}^{X, 1}=K^{X}+\frac{1}{4}\left(f^{\prime}+(n-1) \frac{f}{r}\left(1-\frac{2 m}{r^{n-2}}\right)\right) \square\left(\phi^{2}\right)-\frac{1}{4} \square\left(f^{\prime}+(n-1) \frac{f}{r}\left(1-\frac{2 m}{r^{n-2}}\right)\right) \phi^{2} \\
& =\frac{f^{\prime}}{1-\frac{2 m}{r^{n-2}}}\left(\frac{\partial \phi}{\partial r^{*}}\right)^{2}+\frac{f}{r}\left(1-\frac{n m}{r^{n-2}}\right)|\nabla \phi|_{r^{2} \dot{\gamma}_{n-1}}^{2}-\frac{1}{4} \square\left(f^{\prime}+(n-1) \frac{f}{r}\left(1-\frac{2 m}{r^{n-2}}\right)\right) \phi^{2} .
\end{aligned}
$$

Since, for any function $w$,

$$
\square(w)=\left(g^{-1}\right)^{\mu v} \nabla_{\mu} \partial_{v} w=-\frac{1}{1-\frac{2 m}{r^{n-2}}} \partial_{u^{*}} \partial_{v} * w-\frac{n-1}{2 r}\left(\partial_{u^{*}} w-\partial_{v^{*}} w\right)+\not r_{r^{2} \dot{\gamma}_{n-1}} w,
$$

a straightforward calculation for

$$
w=f^{\prime}+(n-1) \frac{f}{r}\left(1-\frac{2 m}{r^{n-2}}\right)
$$

shows

$$
\begin{aligned}
\square\left(f^{\prime}+(n-1) \frac{f}{r}\left(1-\frac{2 m}{r^{n-2}}\right)\right)= & \frac{1}{1-\frac{2 m}{r^{n-2}}} f^{\prime \prime \prime}+2(n-1) \frac{f^{\prime \prime}}{r}+(n-1)\left[(n-3)+(n-1) \frac{2 m}{r^{n-2}}\right] \frac{f^{\prime}}{r^{2}} \\
& +(n-1)\left[((n-1)(n-2)-(n-3))\left(\frac{2 m}{r^{n-2}}\right)^{2}-n \frac{2 m}{r^{n-2}}-(n-3)\right] \frac{f}{r^{3}} .
\end{aligned}
$$

Thus we finally obtain

$$
\begin{aligned}
K^{X, 1}= & \frac{f^{\prime}}{1-\frac{2 m}{r^{n-2}}}\left(\frac{\partial \phi}{\partial r^{*}}\right)^{2}+\frac{f}{r}\left(1-\frac{n m}{r^{n-2}}\right)|\not \phi|_{r^{2} \dot{\gamma}_{n-1}}^{2}-\frac{1}{4} \frac{f^{\prime \prime \prime}}{1-\frac{2 m}{r^{n-2}}} \phi^{2}-\frac{n-1}{2} \frac{f^{\prime \prime}}{r} \phi^{2} \\
& -\frac{n-1}{4}\left[(n-3)+(n-1) \frac{2 m}{r^{n-2}}\right] \frac{f^{\prime}}{r^{2}} \phi^{2}-\frac{n-1}{4}\left[(n-1)^{2}\left(\frac{2 m}{r^{n-2}}\right)^{2}-n \frac{2 m}{r^{n-2}}-(n-3)\right] \frac{f}{r^{3}} \phi^{2} .
\end{aligned}
$$

Applications of the first modified current. The proofs of Proposition 4.2 and Proposition 4.4 are applications of this formula, as it appears in the energy identity for $J^{X, 1}$ on ${ }^{R} \mathcal{D}_{\tau_{1}}^{\tau_{2}}$; see Appendix B. 
Proof of Proposition 4.4. Choose $f=1$ identically. Then

$$
K^{X, 1}=\frac{1}{r}\left(1-\frac{n m}{r^{n-2}}\right)|\not \nabla|_{r^{2} \dot{\gamma}_{n-1}}^{2}+\frac{n-1}{4}\left[(n-3)+n \frac{2 m}{r^{n-2}}-(n-1)^{2}\left(\frac{2 m}{r^{n-2}}\right)^{2}\right] \frac{1}{r^{3}} \phi^{2} .
$$

Since precisely

$$
\begin{aligned}
g\left(J^{X, 1}, \frac{\partial}{\partial r^{*}}\right)=\frac{1}{4}\left(\frac{\partial \phi}{\partial v^{*}}\right)^{2}+ & \frac{1}{4}\left(\frac{\partial \phi}{\partial u^{*}}\right)^{2}-\frac{1}{2}\left(1-\frac{2 m}{r^{n-2}}\right)|\not \phi|_{r^{2} \dot{\gamma}_{n-1}}^{2} \\
& +\frac{n-1}{2 r}\left(1-\frac{2 m}{r^{n-2}}\right) \phi \frac{\partial \phi}{\partial r^{*}}+\frac{n-1}{4 r^{2}}\left[1-(n-1) \frac{2 m}{r^{n-2}}\right]\left(1-\frac{2 m}{r^{n-2}}\right) \phi^{2},
\end{aligned}
$$

we deduce from the energy identity for $J \frac{\partial}{\partial r^{*}, 1}$ in ${ }^{R} \mathcal{D}_{\tau^{\prime}}^{\tau}$ that

$$
\begin{array}{r}
\left.\int_{R^{*}+2 \tau^{\prime}}^{R^{*}+2 \tau} \int_{\mathbb{S}^{n-1}} \mathrm{~d} \mu_{\dot{\gamma}_{n-1}} r^{n-1}\left\{\frac{1}{4}\left(\frac{\partial \phi}{\partial v^{*}}\right)^{2}+\frac{1}{4}\left(\frac{\partial \phi}{\partial u^{*}}\right)^{2}+\frac{n-1}{4 R^{2}}\left[\frac{1}{2}-(n-1) \frac{2 m}{R^{n-2}}\right]\left(1-\frac{2 m}{R^{n-2}}\right) \phi^{2}\right\}\right|_{r=R} \\
\quad+\int_{R_{\mathcal{D}_{\tau^{\prime}}}} \frac{n-1}{4 r}\left[(n-3)+n \frac{2 m}{r^{n-2}}-(n-1)^{2}\left(\frac{2 m}{r^{n-2}}\right)^{2}\right] \frac{1}{r^{2}} \phi^{2} \mathrm{~d} \mu_{g} \\
\leq\left.\int_{R^{*}+2 \tau^{\prime}}^{R^{*}+2 \tau} \int_{\mathbb{S}^{n-1}} \mathrm{~d} \mu_{\dot{\gamma}_{n-1}} r^{n-1}\left\{\frac{1}{2}\left(1-\frac{2 m}{r^{n-2}}\right)|\not \phi|_{r^{2} \dot{\gamma}_{n-1}}^{2}+\frac{n-1}{2}\left(1-\frac{2 m}{r^{n-2}}\right)\left(\frac{\partial \phi}{\partial r^{*}}\right)^{2}\right\}\right|_{r=R} \\
+C(n, m) \int_{\Sigma_{\tau^{\prime}}}\left(J^{T}(\phi), n\right),
\end{array}
$$

where we have used Proposition C.1 for the boundary terms on $\partial^{R} \mathcal{D}_{\tau^{\prime}}^{\tau} \backslash\{r=R\}$; note that

$$
(n-3)+n \frac{2 m}{r^{n-2}}-(n-1)^{2}\left(\frac{2 m}{r^{n-2}}\right)^{2}>0 \quad(R>\sqrt[n-2]{8 n m}) .
$$

Proof of Proposition 4.2. On one hand we need $f^{\prime}=\mathcal{O}\left(\frac{1}{r^{1+\delta}}\right)$ in view of (4-20), while on the other we already know from the proof of Proposition 4.4 that $f=1$ generates a positive bulk term for $r$ large enough. We choose

$$
f=1-\left(\frac{R}{r}\right)^{\delta}
$$

(where $R>\sqrt[n-2]{2 m}$ is chosen suitably in the last step of the proof) and indeed find

$$
\begin{aligned}
K^{X, 1}= & \delta \frac{R^{\delta}}{r^{1+\delta}}\left(\frac{\partial \phi}{\partial r^{*}}\right)^{2}+\frac{f}{r}\left(1-\frac{n m}{r^{n-2}}\right)|\not \phi|_{r^{2} \dot{\gamma}_{n-1}}^{2} \\
& +\left\{\frac{n-1}{4}(n-3)\left[1-\left(\frac{R}{r}\right)^{\delta}(1+\delta)\right]+\frac{1}{4}\left(\frac{R}{r}\right)^{\delta}[2(n-1)-(2+\delta)] \delta(1+\delta)\right. \\
& +\left[\frac{n-1}{4} n\left[1-\left(\frac{R}{r}\right)^{\delta}\right]-\frac{\delta}{4}\left(\frac{R}{r}\right)^{\delta}\left[n(n+\delta)-2(1+\delta)^{2}\right]\right] \frac{2 m}{r^{n-2}} \\
& \left.-\left[\frac{(n-1)^{3}}{4}\left[1-\left(\frac{R}{r}\right)^{\delta}\right]-\frac{\delta}{4}\left(\frac{R}{r}\right)^{\delta}\left[(n-(1+\delta))(n-1)-\delta^{2}\right]\right]\left(\frac{2 m}{r^{n-2}}\right)^{2}\right\} \frac{1}{r^{3}} \phi^{2} \geq 0
\end{aligned}
$$


for $r \geq R_{1}>R, R_{1}=R_{1}(n, m)>\sqrt[n-2]{2 m}$ chosen large enough. This gives control on $\frac{\partial \phi}{\partial r^{*}}$ and the angular derivatives:

$$
\int_{R_{1} \mathcal{D}_{\tau_{1}}^{\tau_{2}}}\left\{\delta \frac{R^{\delta}}{r^{1+\delta}}\left(\frac{\partial \phi}{\partial r^{*}}\right)^{2}+\frac{f\left(R_{1}\right)}{r}\left(1-\frac{n m}{r^{n-2}}\right)|\not \nabla \phi|_{r^{2} \dot{\gamma}_{n-1}}^{2}\right\} \leq \int_{R_{1} \mathcal{D}_{\tau_{1}}^{\tau_{2}}} K^{X, 1}
$$

Here and in the following, $\tau_{2}>\tau_{1}>\frac{1}{2}\left(t_{0}-R^{*}\right)$. For $\frac{\partial \phi}{\partial t}$ we use the auxiliary current (see also Appendix C)

$$
J_{\mu}^{\mathrm{aux}}=\frac{1}{2}\left(1-\frac{2 m}{r^{n-2}}\right) \delta \frac{R^{\delta}}{r^{1+\delta}} \partial_{\mu}\left(\phi^{2}\right)
$$

to find easily

$$
\begin{aligned}
\int_{R_{1} \mathcal{D}_{\tau_{1}}^{\tau_{2}}} \delta \frac{R^{\delta}}{r^{1+\delta}}\left(\frac{\partial \phi}{\partial t}\right)^{2} \leq \int_{R_{1} \mathcal{D}_{\tau_{1}}^{\tau_{2}}}\{\delta(n+ & \delta) \frac{R^{\delta}}{r^{1+\delta}}\left(\frac{\partial \phi}{\partial r^{*}}\right)^{2} \\
& \left.+\delta \frac{R^{\delta}}{r^{1+\delta}}\left(1-\frac{2 m}{r^{n-2}}\right)|\not \phi|_{r^{2} \dot{\gamma}_{n-1}}^{2}+\delta(n+\delta) \frac{R^{\delta}}{r^{3+\delta}} \phi^{2}+K^{\text {aux }}\right\} .
\end{aligned}
$$

Note that for $r \geq R_{1}$ in particular

$$
\frac{1}{4}[2(n-1)-(2+\delta)] \delta(1+\delta) \frac{R^{\delta}}{r^{3+\delta}} \phi^{2} \leq K^{X, 1} ;
$$

hence

$$
\begin{aligned}
\int_{R_{1} \mathcal{D}_{\tau_{1}}^{\tau_{2}}} \delta & R^{\delta} \\
r^{1+\delta} & \left\{\left(\frac{\partial \phi}{\partial t}\right)^{2}+\left(\frac{\partial \phi}{\partial r^{*}}\right)^{2}\right\} \\
& \leq C(n, m, \delta) \int_{R_{1} \mathcal{D}_{\tau_{1}}^{\tau_{2}}}\left\{K^{X, 1}+K^{\mathrm{aux}}\right\} \\
& \leq C(n, m, \delta) \int_{R_{\mathcal{D}_{\tau_{1}}}^{\tau_{2}}}\left\{K^{X, 1}+K^{\mathrm{aux}}\right\}+C(n, m, \delta) \int_{R_{\mathcal{D}_{\tau_{1}}}^{\tau_{2}} \cap\left\{R<r<R_{1}\right\}}\left\{\frac{1}{r^{1+\delta}}\left(\frac{\partial \phi}{\partial t}\right)^{2}+\frac{1}{r^{3}} \phi^{2}\right\} .
\end{aligned}
$$

By Proposition C.1 (also (B-6)),

$$
\begin{aligned}
\int_{\partial^{R} \mathcal{D}_{\tau_{1}}^{\tau_{2}}}{ }^{*} J^{X, 1} \leq C(n, m, \delta) \int_{\Sigma_{\tau_{1}}} & \left(J^{T}(\phi), n\right)+C(n, m, \delta) \int_{R^{*}+2 \tau_{1}}^{R^{*}+2 \tau_{2}} \mathrm{~d} t \int_{\mathbb{S}^{n-1}} \mathrm{~d} \mu_{\dot{\gamma}_{n-1}} r^{n-1} \times \\
\times & \left.\left\{\frac{1}{2}\left(\frac{\partial \phi}{\partial v^{*}}\right)^{2}+\frac{1}{2}\left(\frac{\partial \phi}{\partial u^{*}}\right)^{2}+\frac{1}{2}\left(1-\frac{2 m}{r^{n-2}}\right)|\not \nabla \phi|_{r^{2} \dot{\gamma}_{n-1}}^{2}+\frac{1}{r^{2}} \phi^{2}\right\}\right|_{r=R}
\end{aligned}
$$

and by Proposition C.8,

$$
\begin{aligned}
\int_{\partial^{R} \mathcal{D}_{\tau_{1}}^{\tau_{2}}}^{*} J^{\text {aux }} \leq C(n, m, \delta) \int_{\Sigma_{\tau_{1}}}\left(J^{T}(\phi), n\right) & \\
& +\int_{R^{*}+2 \tau_{1}}^{R^{*}+2 \tau_{2}} \mathrm{~d} t \int_{\mathbb{S}^{n-1}} \mathrm{~d} \mu_{\dot{\gamma}_{n-1}} r^{n-1}\left\{\frac{\delta}{2} \frac{1}{2}\left[\left(\frac{\partial \phi}{\partial u^{*}}\right)^{2}+\left(\frac{\partial \phi}{\partial v^{*}}\right)^{2}\right]+\frac{\delta}{2} \frac{R^{2 \delta}}{r^{2+2 \delta}} \phi^{2}\right\}_{r=R} .
\end{aligned}
$$


Therefore, by the energy identity for $J^{X, 1}$ and $J^{\text {aux }}$ on ${ }^{R} \mathcal{D}_{\tau_{1}}^{\tau_{2}}$,

$$
\begin{aligned}
\int_{R_{\mathcal{D}_{\tau_{1}}}^{\tau_{2}}}\left\{K^{X, 1}+K^{\mathrm{aux}}\right\} \leq C(n, m, \delta) & \int_{\Sigma_{\tau_{1}}}\left(J^{T}(\phi), n\right)+C(n, m, \delta) \int_{R^{*}+2 \tau_{1}}^{R^{*}+2 \tau_{2}} \mathrm{~d} t \int_{\mathbb{S}^{n-1}} \mathrm{~d} \mu_{\dot{\gamma}_{n-1}} r^{n-1} \times \\
\times & \left.\left\{\frac{1}{2}\left(\frac{\partial \phi}{\partial v^{*}}\right)^{2}+\frac{1}{2}\left(\frac{\partial \phi}{\partial u^{*}}\right)^{2}+\frac{1}{2}\left(1-\frac{2 m}{r^{n-2}}\right)|\not \nabla \phi|_{r^{2} \dot{\gamma}_{n-1}}^{2}+\frac{1}{r^{2}} \phi^{2}\right\}\right|_{r=R} .
\end{aligned}
$$

Our earlier (4-23) derived from the current $J \frac{\partial}{\partial r^{*}}, 1$ now allows us to control the $\frac{\partial \phi}{\partial v^{*}}, \frac{\partial \phi}{\partial u^{*}}$ derivatives and $\phi^{2}$ on the $r=R$ boundary together with the $\phi^{2}$ term in the region $R \leq r \leq R_{1}$ in one step:

$$
\begin{aligned}
& \int_{R_{1} \mathcal{D}_{\tau_{1}}^{\tau_{2}}} \frac{R^{\delta}}{r^{1+\delta}}\left\{\left(\frac{\partial \phi}{\partial t}\right)^{2}+\left(\frac{\partial \phi}{\partial r^{*}}\right)^{2}\right\} \\
& \leq C(n, m, \delta) \int_{\Sigma_{\tau_{1}}}\left(J^{T}(\phi), n\right) \\
& \quad+\left.C(n, m, \delta) \int_{R^{*}+2 \tau_{1}}^{R^{*}+2 \tau_{2}} \mathrm{~d} t \int_{\mathbb{S}^{n-1}} \mathrm{~d} \mu_{\dot{\gamma}_{n-1}} r^{n-1}\left\{\frac{1}{2}\left(1-\frac{2 m}{r^{n-2}}\right)|\not \phi|_{r^{2} \dot{\gamma}_{n-1}}^{2}+\frac{n-1}{2}\left(\frac{\partial \phi}{\partial r^{*}}\right)^{2}\right\}\right|_{r=R} \\
& \quad+C(n, m, \delta) \int_{R_{\mathcal{D}_{\tau_{1}}^{\tau_{2}} \cap\left\{R<r<R_{1}\right\}} \frac{1}{r^{1+\delta}}\left(\frac{\partial \phi}{\partial t}\right)^{2} .}
\end{aligned}
$$

With $t_{0}$ fixed, we can now choose $R$ by Proposition 4.1 such that

$$
\int_{R_{1} \mathcal{D}_{\tau_{1}}^{\tau_{2}}} \frac{1}{r^{1+\delta}}\left\{\left(\frac{\partial \phi}{\partial t}\right)^{2}+\left(\frac{\partial \phi}{\partial r^{*}}\right)^{2}\right\} \leq C(n, m, \delta) \int_{\Sigma_{\tau_{1}}}\left(J^{T}(\phi)+J^{T}(T \cdot \phi), n\right) .
$$

While it is possible to find simple functions $f \geq 0$ to ensure the positivity of $K^{X, 1}$ asymptotically, this is not the case in the entire domain of outer communications; the difficulty is the indefinite sign of (4-20) at the photon sphere $r=\sqrt[n-2]{n m}$, which is a manifestation of the trapping effect.

In the following our strategy will be to prove nonnegativity of $K^{X, 1}$ not pointwise but by using Poincaré inequalities after integration over the spheres (the group orbits of $\mathrm{SO}(n)$ ). This is achieved in two alternative constructions: in Section 4B with a decomposition into spherical harmonics, and in Section $4 \mathrm{C}$ by a commutation with angular momentum operators.

4B. High angular frequencies. Here we construct a positive definite current for the projection of solutions to the wave equation to eigenspaces corresponding to high angular frequencies in the spherical decomposition. Since by Poincaré's inequality the second term in (4-20) then becomes comparable to the zeroth-order terms, the idea is to choose $f$ such that this term dominates. We evidently need

$$
f\left(r^{*}\right) \begin{cases}<0, & r<\sqrt[n-2]{n m} \\ =0, & r=\sqrt[n-2]{n m} \\ >0, & r>\sqrt[n-2]{n m}\end{cases}
$$


and since $f$ should also be bounded one may guess that

$$
f\left(r^{*}\right)=\arctan \left(\frac{(n-1) r^{*}}{\sqrt[n-2]{n m}}\right)
$$

is a good choice; however, while it can ensure positivity at the photon sphere, it fails to do so near the horizon and in the asymptotics. After briefly recalling the spherical decomposition, we will give a more refined construction of $f$, nonetheless guided by the overall characteristics of this function.

Fourier expansion on the sphere $\mathbb{S}^{n-1}$. We recall the Fourier expansion on the sphere $\mathbb{S}^{n-1}$ :

$$
\phi=\sum_{l \geq 0} \pi_{l} \phi, \quad \phi \in \mathrm{L}^{2}\left(\mathbb{S}^{n-1}\right),
$$

where $\pi_{l}$ denotes the orthogonal projection of $\mathrm{L}^{2}\left(\mathbb{S}^{n-1}\right)$ onto $\mathrm{E}_{l}$ (see below):

$$
\stackrel{\otimes}{4-1}_{n-1} \phi=-l(l+n-2) \pi_{l} \phi .
$$

In other words, denoting by $E_{l} \subset \mathrm{L}^{2}\left(\mathbb{S}^{n-1}\right), l \geq 0$, the eigenspaces of

$$
-\AA_{4-1}+\left(\frac{n-2}{2}\right)^{2}
$$

corresponding to the eigenvalue $\left(l+\frac{n-2}{2}\right)^{2}$, then

$$
\mathrm{L}^{2}\left(\mathbb{S}^{n-1}\right)=\bigoplus_{l \geq 0} E_{l}
$$

If we assume $\pi_{l} \phi=0(0 \leq l<L)$ for some $L>0$, then it is easy to show (see, e.g., [Schlue 2012]) that

$$
L(L+n-2) \frac{1}{r^{2}} \int_{S_{r}} \phi^{2} \mathrm{~d} \mu_{\gamma_{r}} \leq \int_{S_{r}}|\nabla \phi|_{r^{2} \dot{\gamma}_{n-1}}^{2} \mathrm{~d} \mu_{\gamma_{r}} ;
$$

this is a well known Poincaré-type inequality on the sphere:

Lemma 4.6 (Poincaré inequality). Let $\phi \in \mathrm{H}^{1}\left(S_{r}\right), S_{r}=\left(\mathbb{S}^{n-1}, r^{2} \dot{\gamma}_{n-1}\right)$, have vanishing projection to $E_{l}, 0 \leq l<L$, for some $L \in \mathbb{N}$; i.e.,

$$
\pi_{l} \phi=0 \quad(0 \leq l<L) .
$$

Then

$$
\int_{S_{r}}|\not \nabla \phi|^{2} \mathrm{~d} \mu_{\gamma_{r}} \geq L(L+n-2) \frac{1}{r^{2}} \int_{S_{r}} \phi^{2} \mathrm{~d} \mu_{\gamma_{r}} .
$$

Construction of the multiplier function for high angular frequencies. The idea is to prescribe the third derivative of $f$ and to find its second and first derivatives by integration with boundary values and parameters that ensure that $f$ remains bounded. Let

$$
\alpha=\frac{n-1}{(n m)^{\frac{1}{n-2}}}
$$


and $\gamma \geq 2, \gamma \in \mathbb{N}$. Consider

$$
f_{\gamma, \alpha}^{\mathrm{III}}\left(r^{*}\right)= \begin{cases}-1, & \left|r^{*}\right| \leq \frac{1}{\gamma \alpha} \\ 1, & \frac{1}{\gamma \alpha}<\left|r^{*}\right| \leq b_{\gamma, \alpha} \\ \left(\frac{b_{\gamma, \alpha}}{r^{*}}\right)^{6}, & \left|r^{*}\right| \geq b_{\gamma, \alpha}\end{cases}
$$

where

$$
b_{\gamma, \alpha}=\frac{5}{6} \frac{2}{\gamma \alpha} \text {. }
$$

Note that $b_{\gamma, \alpha}$ is chosen so that

$$
\int_{0}^{\infty} f_{\gamma, \alpha}^{\mathrm{III}}\left(r^{*}\right) \mathrm{d} r^{*}=0 .
$$

Now define

$$
f_{\gamma, \alpha}^{\mathrm{II}}\left(r^{*}\right)=\int_{0}^{r^{*}} f_{\gamma, \alpha}^{\mathrm{III}}(t) \mathrm{d} t .
$$

Obviously $f_{\gamma, \alpha}^{\mathrm{II}}\left(-r^{*}\right)=-f_{\gamma, \alpha}^{\mathrm{II}}\left(r^{*}\right)$ and, in explicit form,

$$
f_{\gamma, \alpha}^{\mathrm{II}}\left(r^{*}\right)= \begin{cases}-r^{*}, & \left|r^{*}\right| \leq \frac{1}{\gamma \alpha}, \\ r^{*}-\frac{2}{\gamma \alpha}, & \frac{1}{\gamma \alpha}<r^{*} \leq b_{\gamma, \alpha}, \\ r^{*}+\frac{2}{\gamma \alpha}, & -b_{\gamma, \alpha} \leq r^{*}<-\frac{1}{\gamma \alpha}, \\ -\frac{b_{\gamma, \alpha}^{6}}{5 r^{* 5}}, & \left|r^{*}\right| \geq b_{\gamma, \alpha} .\end{cases}
$$

The functions $f_{\gamma, \alpha}^{\mathrm{II}}$ and $f_{\gamma, \alpha}^{\mathrm{III}}$ are sketched in Figure 4.

Next define

$$
f_{\gamma, \alpha}^{\mathrm{I}}=\int_{-\infty}^{r^{*}} f_{\gamma, \alpha}^{\mathrm{II}}(t) \mathrm{d} t .
$$

Here we find

$$
f_{\gamma, \alpha}^{\mathrm{I}}\left(r^{*}\right)= \begin{cases}\frac{b_{\gamma, \alpha}^{6}}{20 r^{* 4}}, & r^{*} \leq-b_{\gamma, \alpha}, \\ \frac{b_{\gamma, \alpha}^{2}}{20}+\frac{1}{2}\left(r^{* 2}-b_{\gamma, \alpha}^{2}\right)+\frac{2}{\gamma \alpha}\left(r^{*}+b_{\gamma, \alpha}\right), & -b_{\gamma, \alpha} \leq r^{*} \leq-\frac{1}{\gamma \alpha}, \\ \frac{13}{12} \frac{1}{(\gamma \alpha)^{2}}-\frac{r^{* 2}}{2}, & -\frac{1}{\gamma \alpha} \leq r^{*} \leq 0,\end{cases}
$$

and $f_{\gamma, \alpha}^{\mathrm{I}}\left(r^{*}\right)=f_{\gamma, \alpha}^{\mathrm{I}}\left(-r^{*}\right)$, as sketched in Figure 5 . 


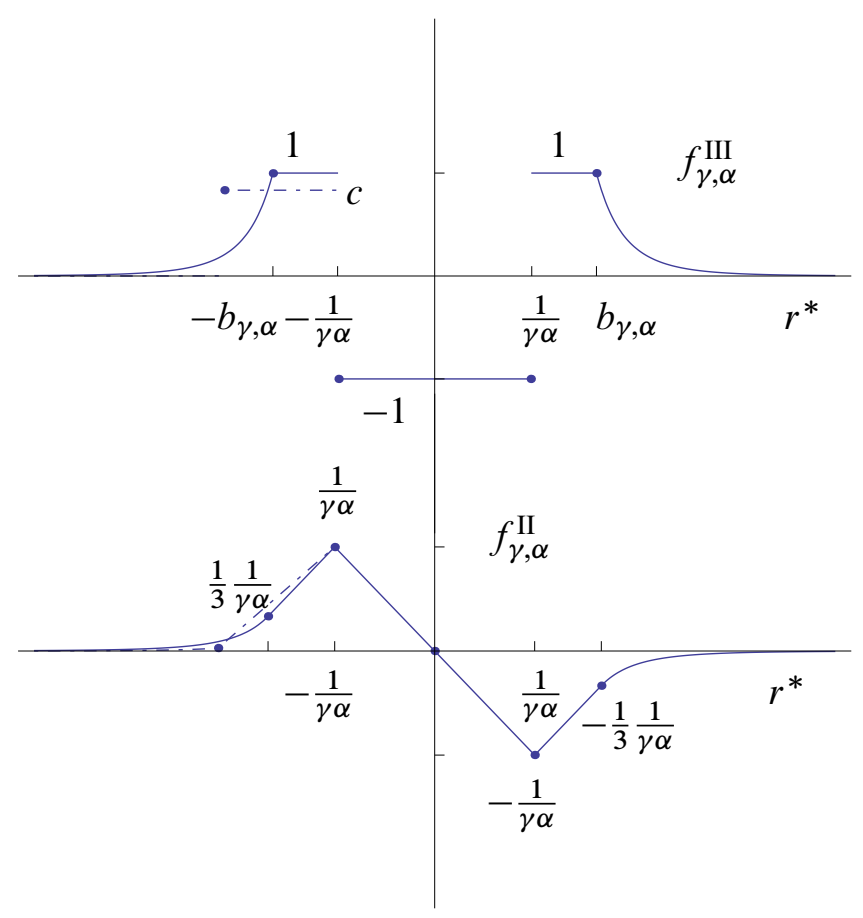

Figure 4. Sketch of the functions $f_{\gamma, \alpha}^{\mathrm{II}}$ and $f_{\gamma, \alpha}^{\mathrm{III}}$, and the adjusted functions (dot-dashed) for $r^{*} \leq 0$.

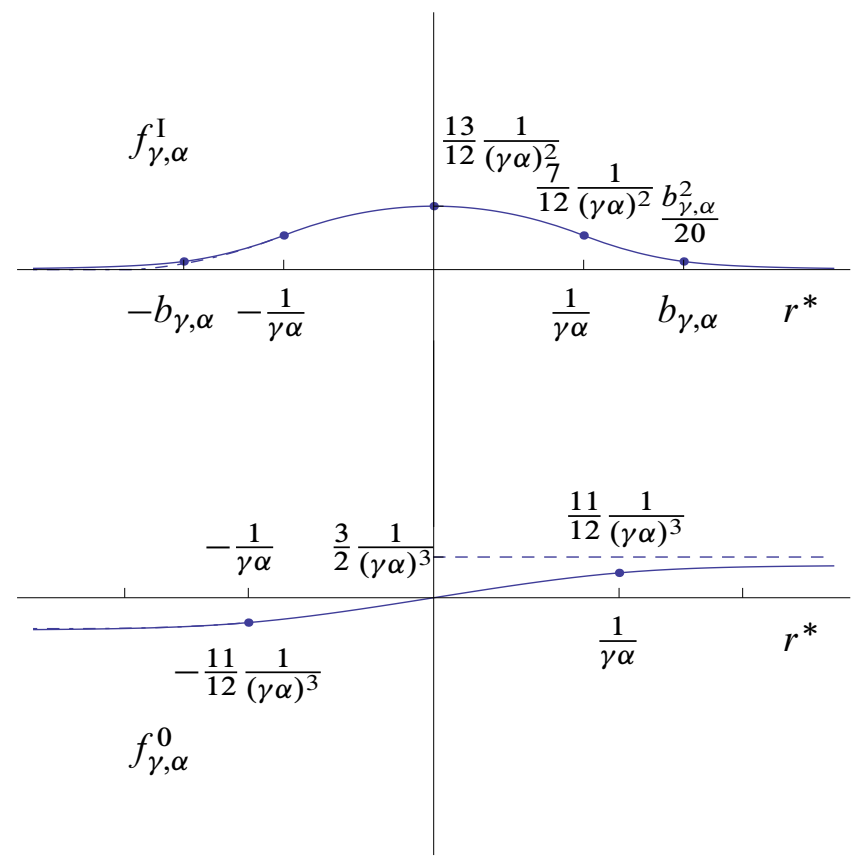

Figure 5. Sketch of the functions $f_{\gamma, \alpha}^{\mathrm{I}}$ and $f_{\gamma, \alpha}^{0}$, and the adjusted functions (dot-dashed) for $r^{*} \leq 0$. 
Finally define

$$
f_{\gamma, \alpha}^{0}\left(r^{*}\right)=\int_{0}^{r^{*}} f_{\gamma, \alpha}^{\mathrm{I}}(t) \mathrm{d} t
$$

Here again $f_{\gamma, \alpha}^{0}\left(-r^{*}\right)=-f_{\gamma, \alpha}^{0}\left(r^{*}\right)$ and, in particular,

$$
f_{\gamma, \alpha}^{0}\left(\frac{1}{\gamma \alpha}\right)=\int_{0}^{\frac{1}{\gamma \alpha}}\left(\frac{13}{12} \frac{1}{(\gamma \alpha)^{2}}-\frac{t^{2}}{2}\right) \mathrm{d} t=\frac{11}{12} \frac{1}{(\gamma \alpha)^{3}} .
$$

Moreover the calculus yields

$$
f\left(b_{\gamma, \alpha}\right)>\frac{1}{(\gamma \alpha)^{3}}, \quad \lim _{r^{*} \rightarrow \infty} f_{\gamma, \alpha}^{0}\left(r^{*}\right)<\frac{3}{2} \frac{1}{(\gamma \alpha)^{3}} .
$$

The function $f_{\gamma, \alpha}^{0}$ is sketched in Figure 5. While this function would suffice in the region $r^{*} \geq-\frac{1}{\gamma \alpha}$ it does not fall-off fast enough as $r^{*} \rightarrow-\infty$.

Lemma 4.7. With $r^{*}$ defined by (2-17) we have, for all $n \geq 3$,

$$
\lim _{r^{*} \rightarrow-\infty}\left(1-\frac{2 m}{r^{n-2}}\right)\left(-r^{*}\right)=0 .
$$

In fact, for all $r^{*}<0$,

$$
\left(1-\frac{2 m}{r^{n-2}}\right) \leq \frac{(2 m)^{\frac{1}{n-2}}}{\left(-r^{*}\right)}
$$

Proof. See Appendix B.

Next we will make an adjustment to $f^{\mathrm{III}}$ on $r^{*} \leq 0$ that introduces faster decay while keeping the area under the graph of $f^{\mathrm{III}}$ and $f^{\mathrm{II}}$ fixed [Schlue 2012]. In other words, there are constants

$$
b_{\gamma, \alpha} \leq b \leq \frac{4}{\gamma \alpha}, \quad \frac{1}{4} \leq c \leq 1
$$

such that, if we redefine $f_{\gamma, \alpha}^{\mathrm{III}}$ for $r^{*} \leq 0$ as

$$
f_{\gamma, \alpha}^{\mathrm{III}}\left(r^{*}\right)= \begin{cases}-1, & -\frac{1}{\gamma \alpha} \leq r^{*} \leq 0, \\ c, & -b \leq r^{*} \leq \frac{1}{\gamma \alpha}, \\ \left(1-\frac{2 m}{r^{n-2}}\right)^{6}\left(\frac{b}{(2 m)^{\frac{1}{n-2}}}\right)^{6}, & r^{*} \leq-b,\end{cases}
$$

then

$$
\int_{0}^{-\infty} f_{\gamma, \alpha}^{\mathrm{III}}\left(r^{*}\right) \mathrm{d} r^{*}=0, \quad \int_{-\infty}^{0} \int_{0}^{r^{*}} f_{\gamma, \alpha}^{\mathrm{III}}(t) \mathrm{d} t \mathrm{~d} r^{*}=\int_{-\infty}^{0} \int_{0}^{-r^{*}}\left(-f_{\gamma, \alpha}^{\mathrm{III}}(t)\right) \mathrm{d} t \mathrm{~d} r^{*} .
$$

The adjusted functions in comparison to the old are also sketched in Figures 4 and 5. Note in particular 
that, for $r^{*} \leq 0$,

and, for $r^{*} \leq-\frac{1}{\gamma \alpha}$

$$
\begin{gathered}
f^{\mathrm{II}}\left(r^{*}\right) \leq \frac{1}{\gamma \alpha}, \\
f^{\mathrm{I}}\left(r^{*}\right) \leq f^{\mathrm{I}}\left(-r^{*}\right) \leq \frac{13}{12} \frac{1}{(\gamma \alpha)^{2}},
\end{gathered}
$$

$$
\frac{11}{12} \frac{1}{(\gamma \alpha)^{3}} \leq\left|f^{0}\left(r^{*}\right)\right| \leq f^{0}\left(-r^{*}\right)<\frac{3}{2} \frac{1}{(\gamma \alpha)^{3}} .
$$

Remark 4.8. In order to deal with smooth functions one could use (e.g., at the level of second derivatives) a convolution with a Gaussian on the scale given by $\gamma \alpha$ (or finer); i.e., one could define

$$
f_{\gamma, \alpha}^{\prime \prime}\left(r^{*}\right)=\frac{\gamma \alpha}{\sqrt{\pi}} \int_{-\infty}^{\infty} e^{-(\gamma \alpha)^{2}\left(r^{*}-t\right)^{2}} f_{\gamma, \alpha}^{\mathrm{II}}(t) \mathrm{d} t
$$

and find $f_{\gamma, \alpha}^{\prime \prime \prime}=\frac{\mathrm{d}}{\mathrm{d} r^{*}} f_{\gamma, \alpha}^{\prime \prime}$ by differentiation, and $f_{\gamma, \alpha}^{\prime}$ and $f_{\gamma, \alpha}$ by integration with the boundary values $f_{\gamma, \alpha}^{\prime}(-\infty)=0, f_{\gamma, \alpha}(0)=0$ as above. However, we choose not to do so (as it does not give further insight) and work directly with the step-functions, i.e., define

$$
f_{\gamma, \alpha}^{\prime \prime}=f_{\gamma, \alpha}^{\mathrm{III}}
$$

We are now in the position to prove a nonnegativity property of the terms occurring in (4-20), which we will denote by ${ }^{0} K^{X, 1}$ :

$$
K^{X, 1}=\frac{f^{\prime}}{1-\frac{2 m}{r^{n-2}}}\left(\frac{\partial \phi}{\partial r^{*}}\right)^{2}+{ }^{0} K^{X, 1} .
$$

Proposition 4.9 (positivity of the current $J^{X_{\gamma, \alpha}, 1}$ ). For $n \geq 3$,

$$
X_{\gamma, \alpha}=f_{\gamma, \alpha} \frac{\partial}{\partial r^{*}} \quad(\text { where we choose } \gamma=12)
$$

and $\phi \in \mathrm{H}^{1}(S)$ satisfy

$$
\int_{S}{ }^{0} K^{X_{\gamma, \alpha}, 1} \mathrm{~d} \mu_{\gamma} \geq 0
$$

provided $\pi_{l} \phi=0$ for $0 \leq l<L$, where $L \geq(6 \gamma n)^{2}$ is fixed.

Proof. By Lemma 4.6,

$$
\begin{aligned}
\int_{S}{ }^{0} K^{X_{\gamma, \alpha}, 1} \mathrm{~d} \mu_{\gamma} \geq \int_{S}\{L(L & +n-2) \frac{f_{\gamma, \alpha}}{r^{3}}\left(1-\frac{n m}{r^{n-2}}\right) \\
& -\frac{1}{4} \frac{f_{\gamma, \alpha}^{\prime \prime \prime}}{1-\frac{2 m}{r^{n-2}}}-\frac{n-1}{2} \frac{f_{\gamma, \alpha}^{\prime \prime}}{r}-\frac{n-1}{4}\left[(n-3)+(n-1) \frac{2 m}{r^{n-2}}\right] \frac{f_{\gamma, \alpha}^{\prime}}{r^{2}} \\
& \left.-\frac{n-1}{4}\left[(n-1)^{2}\left(\frac{2 m}{r^{n-2}}\right)^{2}-n \frac{2 m}{r^{n-2}}-(n-3)\right] \frac{f_{\gamma, \alpha}}{r^{3}}\right\} \phi^{2} \mathrm{~d} \mu_{\gamma} .
\end{aligned}
$$


We consider the five regions

$$
-\infty<-\frac{4}{\gamma \alpha}<-\frac{1}{\gamma \alpha}<\frac{1}{\gamma \alpha}<b_{\gamma, \alpha}<\infty .
$$

The proofs of the following four lemmas are omitted here; see [Schlue 2012].

Step 1 (near the photon sphere, $\left|r^{*}\right|<\frac{1}{\gamma \alpha}$ ).

Lemma 4.10. In the region $\left|r^{*}\right|<\frac{1}{\gamma \alpha}$ the corresponding value of $r$ lies in the interval

$$
\sqrt[n-2]{\delta n m}<r<\frac{n}{\alpha}
$$

where $\delta=\max \left\{\frac{1}{3}, \frac{4}{3} \frac{2}{n}\right\}$.

Recalling $f_{\gamma, \alpha}$ and its derivatives, we then find, in the region $\left|r^{*}\right|<\frac{1}{\gamma \alpha}$,

$$
\begin{aligned}
& \int_{S}{ }^{0} K^{X_{\gamma, \alpha}, 1} \mathrm{~d} \mu_{\gamma} \\
& \geq \int_{S}\left\{\frac{1}{4}-\frac{1}{2} \frac{\alpha}{\delta \frac{1}{n-2}} \frac{1}{\gamma \alpha}-\frac{1}{4} \frac{\alpha^{2}}{\delta \frac{2}{n-2}} \frac{1}{n-1}\left[(n-3)+\frac{1}{\delta}(n-1) \frac{2}{n}\right] \frac{13}{12} \frac{1}{(\gamma \alpha)^{2}}-\frac{1}{4} \frac{\alpha^{3}}{\delta \frac{3}{n-2}} \frac{2}{\delta n} \frac{3}{2} \frac{1}{(\gamma \alpha)^{3}}\right\} \phi^{2} \mathrm{~d} \mu_{\gamma} \\
& \geq \int_{S}\left\{\frac{1}{4}-\frac{1}{2} \frac{3}{\gamma}-\frac{3}{4} \frac{13}{12}\left(\frac{3}{\gamma}\right)^{2}-\frac{3}{4}\left(\frac{3}{\gamma}\right)^{3}\right\} \phi^{2} \mathrm{~d} \mu_{\gamma} \geq \int_{S} \frac{1}{4} \frac{1}{8} \phi^{2} \mathrm{~d} \mu_{\gamma}
\end{aligned}
$$

because $\gamma=12$.

Step 2 (in the intermediate region, $\frac{1}{\gamma \alpha} \leq r^{*} \leq \frac{5}{6} \frac{2}{\gamma \alpha}$ ).

Lemma 4.11. In the region $\frac{1}{\gamma \alpha} \leq r^{*} \leq \frac{5}{6} \frac{2}{\gamma \alpha}$ we have, for the corresponding value of $r$,

$$
\left(1+\frac{1}{3 \gamma(n-1)}\right)(n m)^{\frac{1}{n-2}} \leq r \leq \frac{n}{\alpha} .
$$

Collecting the first term and the last, we find in this region,

$$
\begin{aligned}
\int_{S}{ }^{0} K^{X_{\gamma, \alpha}, 1} \mathrm{~d} \mu_{\gamma} & \geq \int_{S}\left\{\frac{\alpha^{3}}{n^{3}} \frac{11}{12} \frac{1}{(\gamma \alpha)^{3}}\left[\left(1-\frac{n m}{r^{n-2}}\right) L(L+n-2)-\frac{n-1}{4}(n-1)^{2}\left(\frac{2}{n}\right)^{2}+\frac{1}{4}(n-1)(n-3)\right]\right. \\
& \left.-\frac{1}{4} \frac{1}{1-\frac{2}{n}}+\frac{\alpha}{3} \frac{1}{3} \frac{1}{\gamma \alpha}-\frac{1}{4} \alpha^{2} \frac{1}{n-1}\left[(n-3)+(n-1) \frac{2}{n}\right] \frac{1}{(\gamma \alpha)^{2}}\right\} \phi^{2} \mathrm{~d} \mu_{\gamma} \\
& \geq \int_{S}\left\{\frac{11}{12} \frac{1}{(n \gamma)^{3}}\left[\frac{1}{6 \gamma(n-1)} L(L+n-2)-(n-1)+\frac{1}{4}(n-1)(n-3)\right]-\frac{3}{4}-\frac{1}{4} \frac{1}{\gamma^{2}}\right\} \phi^{2} \mathrm{~d} \mu_{\gamma} \\
& \geq \int_{S}\left\{\frac{11}{12} \frac{1}{6}\left(\frac{(6 \gamma n)^{2}}{\gamma^{2} n^{2}}\right)^{2}-1\right\} \phi^{2} \mathrm{~d} \mu_{\gamma} \geq \int_{S} \phi^{2} \mathrm{~d} \mu_{\gamma}
\end{aligned}
$$

because $L \geq(6 \gamma n)^{2}$, where we have used that, for $\frac{1}{\gamma \alpha} \leq r^{*} \leq \frac{5}{6} \frac{2}{\gamma \alpha}$,

$$
1-\frac{n m}{r^{n-2}} \geq \frac{1}{6 \gamma(n-1)} \text {. }
$$


Step 3 (in the asymptotics, $r^{*} \geq b_{\gamma, \alpha}$ ). Given the general fact Proposition B.1 we here only need the weaker statement:

Lemma 4.12. For $r^{*} \geq \frac{5}{6} \frac{2}{\gamma \alpha}$,

$$
\frac{r}{r^{*}} \leq 2 \gamma n
$$

Here

$$
\begin{aligned}
\int_{S}{ }^{0} K^{X_{\gamma, \alpha}, 1} \mathrm{~d} \mu_{\gamma} & \geq \int_{S}\left\{\frac{1}{(\gamma \alpha)^{3}}\left[\frac{1}{6 \gamma(n-1)} L(L+n-2)-\frac{3}{2}(n-1)\right] \frac{1}{r^{3}}-\frac{1}{4} \frac{1}{1-\frac{2}{n}\left(\frac{5}{6} \frac{2}{\gamma \alpha r^{*}}\right)^{6}}\right. \\
& \geq \int_{S}\left[\frac{L^{2}}{6 \gamma^{4} n}+\frac{L}{6 \gamma^{4} n}(n-2)-\frac{3}{2} \frac{1}{\gamma^{3}}(n-1)-\frac{3}{4}\left(\frac{r}{r^{*}}\right)^{3}\left(\frac{5}{6} \frac{2}{\gamma}\right)^{6} \frac{1}{\left(\alpha r^{*}\right)^{3}}\right. \\
& \left.\left.\geq \int_{S}\left[(6 n)^{3}-(4 n)^{3}\right] \frac{1}{(\alpha r)^{3}} \phi^{2} \mathrm{~d} \mu_{\gamma} \geq \int_{S}\left(\frac{n}{\alpha r}\right)^{3} \phi^{2} \mathrm{~d} \mu_{\gamma} \geq \int_{S}\left(\frac{\left.\frac{5}{6} \frac{2}{\gamma \alpha}\right)^{6}}{r^{*} 4}\right\}^{2} \mathrm{~d} \mu_{\gamma}\left(\frac{r}{r^{*}}\right)^{3}\left(\frac{5}{6} \frac{2}{\gamma}\right)^{6} \frac{1}{\alpha r^{*}}\right] \frac{1}{(\alpha r)^{3}} \phi^{2} \mathrm{~d} \mu_{\gamma}\right)^{\frac{1}{n-2}} \phi^{2} \mathrm{~d} \mu_{\gamma}
\end{aligned}
$$

where in the third bound we have again used $L \geq(6 \gamma n)^{2}$ and the lemma.

Step 4 (in the intermediate region, $-\frac{4}{\gamma \alpha} \leq r^{*} \leq-\frac{1}{\gamma \alpha}$ ). Recall $\gamma=12$.

Lemma 4.13. For $k \leq \gamma, k \in \mathbb{N}$,

and, consequently,

$$
\left.\left(1-\frac{2 m}{r^{n-2}}\right)^{-1}\right|_{r^{*}=-\frac{k}{\gamma \alpha}} \leq 17,
$$

$$
-\left.\left(1-\frac{n m}{r^{n-2}}\right)\right|_{r^{*}=-\frac{1}{\gamma \alpha}} \geq \frac{1}{20} \frac{1}{2 \gamma} .
$$

In the region $-\frac{4}{\gamma \alpha} \leq r^{*} \leq-\frac{1}{\gamma \alpha}$ we directly apply the lemma to see that $\int_{S}{ }^{0} K^{X_{\gamma, \alpha}, 1} \mathrm{~d} \mu_{\gamma}$

$$
\begin{aligned}
& \geq \int_{S}\left\{L(L+n-2) \frac{1}{(n m)^{\frac{3}{n-2}}} \frac{11}{12} \frac{1}{(\gamma \alpha)^{3}} \frac{1}{20} \frac{1}{2 \gamma}-\frac{1}{4} 17-\frac{n-1}{2} \frac{1}{(2 m)^{\frac{1}{n-2}}} \frac{1}{\gamma \alpha}\right. \\
& \left.\quad-\frac{n-1}{2}[(n-3)+(n-1)] \frac{1}{(2 m)^{\frac{2}{n-2}}} \frac{13}{12} \frac{1}{(\gamma \alpha)^{2}}-\frac{n-1}{4}[n+(n-3)] \frac{1}{(2 m)^{\frac{3}{n-2}}} 2 \frac{1}{(\gamma \alpha)^{3}}\right\} \phi^{2} \mathrm{~d} \mu_{\gamma} \\
& \geq \int_{S}\left\{\frac{1}{(3 \gamma)^{4}} \frac{1}{(n-1)^{3}} L(L+n-2)-\frac{17}{4}-\frac{3}{2} \frac{1}{2 \gamma}-\frac{13}{12} \frac{1}{\gamma^{2}}\left(\frac{n}{2}\right)^{\frac{2}{n-2}}-\frac{1}{n-1} \frac{1}{\gamma^{3}}\left(\frac{n}{2}\right)^{\frac{3}{n-2}}\right\} \phi^{2} \mathrm{~d} \mu_{\gamma} \\
& \geq \int_{S}\left\{2^{4} n-\frac{23}{4}\right\} \phi^{2} \mathrm{~d} \mu_{\gamma} \geq \int_{S} \phi^{2} \mathrm{~d} \mu_{\gamma},
\end{aligned}
$$


because $L \geq(6 \gamma n)^{2}$.

Step 5 (near the horizon, $r^{*} \leq-b$ ). Finally we see for $r^{*} \leq-b$, recalling the adjustment to faster fall-off,

$$
\begin{aligned}
& \int_{S}{ }^{0} K^{X_{\gamma, \alpha}, 1} \mathrm{~d} \mu_{\gamma} \\
& \geq \int_{S}\left\{L(L+n-2) \frac{1}{(n m)^{\frac{3}{n-2}}} \frac{11}{12} \frac{1}{(\gamma \alpha)^{3}} \frac{1}{20} \frac{1}{2 \gamma}-\frac{1}{4}\left(1-\frac{2}{n}\right)^{5}-\frac{n-1}{2} \frac{1}{(2 m)^{\frac{1}{n-2}}} \frac{1}{\gamma \alpha}\right. \\
& \left.\quad-\frac{(n-1)^{2}}{4} \frac{1}{(2 m)^{\frac{1}{n-2}}} \frac{1}{(\gamma \alpha)^{2}}-\frac{n-1}{4}[n+(n-3)] \frac{1}{(2 m)^{\frac{3}{n-2}}} 2 \frac{1}{(\gamma \alpha)^{3}}\right\} \phi^{2} \mathrm{~d} \mu_{\gamma} \\
& \geq \int_{S}\left\{\frac{1}{(3 \gamma)^{4}} \frac{1}{(n-1)^{3}} L(L+n-2)-\frac{1}{4}-\frac{1}{2 \gamma}\left(\frac{n}{2}\right)^{\frac{1}{n-2}}-\frac{1}{(2 \gamma)^{2}}\left(\frac{n}{2}\right)^{\frac{2}{n-2}}-\frac{4}{n-1} \frac{1}{(2 \gamma)^{3}}\left(\frac{n}{2}\right)^{\frac{3}{n-2}}\right\} \phi^{2} \mathrm{~d} \mu_{\gamma} \\
& \geq \int_{S}\left\{2^{4} n-\frac{5}{4}\right\} \phi^{2} \mathrm{~d} \mu_{\gamma} \geq \int_{S} \phi^{2} \mathrm{~d} \mu_{\gamma},
\end{aligned}
$$

where we have used that here

$$
\frac{f^{\prime \prime \prime}}{1-\frac{2 m}{r^{n-2}}}=\left(1-\frac{2 m}{r^{n-2}}\right)^{5}\left(\frac{b}{(2 m)^{\frac{1}{n-2}}}\right)^{6} \leq\left(1-\frac{2}{n}\right)^{5} \leq 1 .
$$

In fact, we have shown more, because all lower bounds in Steps 1-5 are minorized by $\frac{1}{4} \frac{1}{8} \frac{(2 m)^{\frac{3}{n-2}}}{r^{3}}$.

Corollary 4.14. Let $\phi$ be a solution of the wave equation $\square_{g} \phi=0$ satisfying

$$
\pi_{l} \phi=0 \quad(0 \leq l<L)
$$

on the standard sphere $S=\left(\mathbb{S}^{n-1}, r^{2} \dot{\gamma}_{n-1}\right)$ for a fixed $L \geq(6 \gamma n)^{2}$. Then

$$
\int_{S}\left\{\frac{1}{4} \frac{1}{8} \frac{(2 m)^{\frac{3}{n-2}}}{r^{3}} \phi^{2}+\frac{1}{\left(20 \gamma^{2}\right)^{3}} \frac{1}{(n-2)^{2}(n-1)^{6}}\left(1-\frac{2 m}{r^{n-2}}\right)^{5} \frac{(2 m)^{\frac{6}{n-2}}}{r^{4}}\left(\frac{\partial \phi}{\partial r^{*}}\right)^{2}\right\} \mathrm{d} \mu_{\gamma} \leq \int_{S} K^{X_{\gamma, \alpha}, 1} \mathrm{~d} \mu_{\gamma} .
$$

Proof. It remains to be shown that

$$
\frac{1}{20} \frac{1}{(4 \cdot 5(n-2))^{2}}\left(1-\frac{2 m}{r^{n-2}}\right)^{6} \frac{b_{\gamma, \alpha}^{6}}{r^{4}} \leq f_{\gamma, \alpha}^{\prime} .
$$

First,

$$
\int_{-\infty}^{r^{*}}\left(1-\frac{2 m}{r^{n-2}}\right)^{6} \mathrm{~d} r^{*}=\int_{(2 m)^{\frac{1}{n-2}}}^{r}\left(1-\frac{2 m}{r^{n-2}}\right)^{5} \mathrm{~d} r
$$

because $\mathrm{d} r^{*} / \mathrm{d} r=\left(1-\frac{2 m}{r^{n-2}}\right)^{-1}$. Now choose $\sqrt[n-2]{2 m}<r_{0}<r$ so close to $r$ as to satisfy

$$
\frac{r-r_{0}}{r_{0}}=\frac{1}{2} \frac{1}{5(n-2)}\left(1-\frac{2 m}{r^{n-2}}\right) \text {. }
$$


Then, by the mean value theorem,

$$
\begin{aligned}
\int_{(2 m) \frac{1}{n-2}}^{r}\left(1-\frac{2 m}{r^{n-2}}\right)^{5} \mathrm{~d} r & \geq\left(1-\frac{2 m}{r_{0}^{n-2}}\right)^{5}\left(r-r_{0}\right) \geq\left(1-\frac{2 m}{r^{n-2}}\right)^{5}\left[1-5(n-2) \frac{1}{1-\frac{2 m}{r^{n-2}}} \frac{r-r_{0}}{r_{0}}\right]\left(r-r_{0}\right) \\
& \geq \frac{1}{4} \frac{1}{5(n-2)}\left(1-\frac{2 m}{r^{n-2}}\right)^{6}(2 m)^{\frac{1}{n-2}}
\end{aligned}
$$

We conclude, for $r^{*} \leq-b$,

$$
\begin{aligned}
f_{\gamma, \alpha}^{\prime}\left(r^{*}\right) & =\left.\int_{-\infty}^{r^{*}} \int_{-\infty}^{s^{*}}\left(1-\frac{2 m}{r^{n-2}}\right)\right|_{r^{*}=s^{*}}\left(\frac{b}{(2 m)^{\frac{1}{n-2}}}\right)^{6} \mathrm{~d} s^{*} \mathrm{~d} r^{*} \geq \frac{1}{4} \frac{1}{5(n-2)} \int_{-\infty}^{r^{*}}\left(1-\frac{2 m}{r^{n-2}}\right)^{6} \mathrm{~d} r^{*} \frac{b^{6}}{(2 m)^{\frac{5}{n-2}}} \\
& \geq\left(\frac{1}{4} \frac{1}{5(n-2)}\right)^{2}\left(1-\frac{2 m}{r^{n-2}}\right)^{6} \frac{b^{6}}{(2 m)^{\frac{4}{n-2}}} \geq \frac{1}{(4 \cdot 5(n-2))^{2}}\left(1-\frac{2 m}{r^{n-2}}\right)^{6} \frac{b_{\gamma, \alpha}^{6}}{r^{4}}
\end{aligned}
$$

Second, for $r^{*} \geq 0$,

$$
\frac{1}{(4 \cdot 5(n-2))^{2}} \frac{1}{r^{4}}=\frac{1}{(4 \cdot 5(n-2))^{2}}\left(\frac{r^{*}}{r}\right)^{4} \frac{1}{r^{* 4}} \leq \frac{1}{r^{* 4}} .
$$

Since, thirdly,

$$
\frac{b_{\gamma, \alpha}}{r} \leq 1,
$$

we have established (*) for the regions $r^{*} \leq-b, r^{*} \geq b_{\gamma, \alpha},-b \leq r^{*} \leq b_{\gamma, \alpha}$, respectively.

Remark 4.15. This estimate of the zeroth-order term $\phi^{2}$ suffices to obtain an estimate for all derivatives using a commutation with the vector field $T$; see the proof of Proposition 4.1 in Section 4D.1.

4C. Low angular frequencies and commutation. While the current constructed in Section 4B required a decomposition into spherical harmonics, we will now altogether avoid a recourse to the Fourier expansion on the sphere. The key to the positivity property was Poincaré's inequality, which states in more generality:

Lemma 4.16 (Poincaré inequality). Let $(S, \gamma)$ be a compact Riemannian manifold, and $\phi \in \mathrm{H}^{1}(S) a$ function on $S$ with mean value

$$
\bar{\phi}=\frac{1}{\int_{S} \mathrm{~d} \mu_{\gamma}} \int_{S} \phi \mathrm{d} \mu_{\gamma}
$$

Then

$$
\int_{S}(\phi-\bar{\phi})^{2} \mathrm{~d} \mu_{\gamma} \leq \frac{1}{\lambda_{1}(S)} \int_{S}|\not \nabla|^{2} \mathrm{~d} \mu_{\gamma}
$$

where $\lambda_{1}(S)$ is the first nonzero eigenvalue of the negative Laplacian, $-\not \nabla=-\nabla^{a} \nabla_{a}$, on $S$ ( $\mathbb{\nabla}$ denotes covariant differentiation on $S$ ).

Now let $(S, \gamma)=\left(\mathbb{S}^{n-1}, \stackrel{\circ}{\gamma}_{n-1}\right)$. Then we read off from (4-27) here

$$
\lambda_{1}\left(\mathbb{S}^{n-1}\right)=n-1 .
$$


Choose a basis of the Lie algebra of $\mathrm{SO}(n)$,

$$
\Omega_{i}: i=1, \ldots, \frac{n(n-1)}{2},
$$

and apply Lemma 4.16 to the functions $\Omega_{i} \phi$ of vanishing mean:

$$
\int_{\mathbb{S}^{n-1}} \Omega_{i} \phi \mathrm{d} \mu_{\dot{\gamma}_{n-1}}=0 .
$$

Then we obtain

$$
\int_{\mathbb{S}^{n-1}}\left|\nabla \Omega_{i} \phi\right|^{2} \mathrm{~d} \mu_{\dot{\gamma}_{n-1}} \geq(n-1) \int_{\mathbb{S}^{n-1}}\left(\Omega_{i} \phi\right)^{2} \mathrm{~d} \mu_{\dot{\gamma}_{n-1}}
$$

or, on $(S, \gamma)=\left(S_{r}, \gamma_{r}\right)=\left(\mathbb{S}^{n-1}, r^{2} \dot{\circ}_{n-1}\right)$,

$$
\int_{S_{r}}\left|\not \nabla \Omega_{i} \phi\right|^{2} \mathrm{~d} \mu_{\gamma_{r}} \geq \frac{n-1}{r^{2}} \int_{S_{r}}\left(\Omega_{i} \phi\right)^{2} \mathrm{~d} \mu_{\gamma_{r}}
$$

Also note

$$
\sum_{i=1}^{\frac{n(n-1)}{2}}\left(\Omega_{i} \phi\right)^{2}=r^{2}|\not \phi|_{r^{2} \dot{\gamma}_{n-1}}^{2}
$$

Second modified current. Recall we are considering vector fields of the form

$$
X=f\left(r^{*}\right) \frac{\partial}{\partial r^{*}} .
$$

Define

$$
J_{\mu}^{X, 2}=J_{\mu}^{X, 1}+\frac{f^{\prime}}{f\left(1-\frac{2 m}{r^{n-2}}\right)} \beta X_{\mu} \phi^{2},
$$

where $\beta=\beta\left(r^{*}\right)$ is a function to be chosen below. Then

$$
\begin{aligned}
K^{X, 2}= & K^{X, 1}+\nabla^{\mu}\left(\frac{f^{\prime}}{f\left(1-\frac{2 m}{r^{n-2}}\right)} \beta X_{\mu} \phi^{2}\right) \\
= & \frac{f^{\prime}}{1-\frac{2 m}{r^{n-2}}}\left(\frac{\partial \phi}{\partial r^{*}}+\beta \phi\right)^{2}+\frac{f}{r}\left(1-\frac{n m}{r^{n-2}}\right)|\nabla \phi|_{r^{2} \dot{\gamma}_{n-1}}^{2} \\
& -\frac{1}{4} \frac{f^{\prime \prime \prime}}{1-\frac{2 m}{r^{n-2}}} \phi^{2}+\frac{f^{\prime \prime}}{1-\frac{2 m}{r^{n-2}}}\left[\beta-\frac{n-1}{2 r}\left(1-\frac{2 m}{r^{n-2}}\right)\right] \phi^{2} \\
& -\frac{f^{\prime}}{1-\frac{2 m}{r^{n-2}}}\left[\beta^{2}-\beta^{\prime}-\frac{n-1}{r} \beta\left(1-\frac{2 m}{r^{n-2}}\right)+\frac{n-1}{4 r^{2}}\left((n-3)+(n-1) \frac{2 m}{r^{n-2}}\right)\left(1-\frac{2 m}{r^{n-2}}\right)\right] \phi^{2} \\
& -\frac{n-1}{4}\left[(n-1)^{2}\left(\frac{2 m}{r^{n-2}}\right)-n \frac{2 m}{r^{n-2}}-(n-3)\right] \frac{f}{r^{3}} \phi^{2}
\end{aligned}
$$

Now choose

$$
\beta=\frac{n-1}{2 r}\left(1-\frac{2 m}{r^{n-2}}\right)+\delta
$$


Then

$$
\beta^{2}-\beta^{\prime}-\frac{n-1}{r} \beta\left(1-\frac{2 m}{r^{n-2}}\right)+\frac{n-1}{4 r^{2}}\left((n-3)+(n-1) \frac{2 m}{r^{n-2}}\right)\left(1-\frac{2 m}{r^{n-2}}\right)=-\delta^{\prime}+\delta^{2}
$$

and

$$
\begin{aligned}
K^{X, 2}=\frac{f^{\prime}}{1-\frac{2 m}{r^{n-2}}}\left(\frac{\partial \phi}{\partial r^{*}}+\beta \phi\right)^{2}+\frac{f}{r}\left(1-\frac{n m}{r^{n-2}}\right) & |\not \phi|_{r^{2} \dot{\gamma}_{n-1}}^{2}-\frac{1}{1-\frac{2 m}{r^{n-2}}}\left\{\frac{1}{4} f^{\prime \prime \prime}-\delta f^{\prime \prime}+\left(\delta^{2}-\delta^{\prime}\right) f^{\prime}\right\} \phi^{2} \\
& -\frac{n-1}{4}\left[(n-1)^{2}\left(\frac{2 m}{r^{n-2}}\right)^{2}-n \frac{2 m}{r^{n-2}}-(n-3)\right] \frac{f}{r^{3}} \phi^{2} .
\end{aligned}
$$

Note. Suppose, outside a compact interval $[-\alpha, \alpha] \subset \mathbb{R}, f^{\prime}$ is of the form $f^{\prime}\left(r^{*}\right)=\frac{1}{r^{* 2}}\left(\left|r^{*}\right|>\alpha\right)$. Then we could choose $\delta=-\frac{1}{r^{*}}\left(\left|r^{*}\right|>\alpha\right)$ so that $\delta f^{\prime \prime}=\frac{2}{r^{* 4}} \geq 0$ and $-\delta^{\prime}+\delta^{2}=0$.

Definition of the current $J^{(\alpha)}$. Let $\alpha>0$ and introduce a shifted coordinate

$$
x=r^{*}-\alpha-\sqrt{\alpha} .
$$

The modification we choose is

$$
\delta=-\frac{x}{\alpha^{2}+x^{2}}
$$

so that

$$
-\delta^{\prime}+\delta^{2}=\frac{\alpha^{2}}{\left(\alpha^{2}+x^{2}\right)^{2}}
$$

Let

$$
f^{a}=-\frac{C}{\alpha^{2} r^{n-1}} \quad(C>0)
$$

and

$$
\left(f^{b}\right)^{\prime}=\frac{1}{\alpha^{2}+x^{2}}, \quad\left(f^{b}\right)\left(r^{*}\right)=\int_{0}^{r^{*}} \frac{1}{\alpha^{2}+x\left(t^{*}\right)^{2}} \mathrm{~d} t^{*}
$$

Note that then

$$
\left(f^{a}\right)^{\prime}+(n-1) \frac{f^{a}}{r}\left(1-\frac{2 m}{r^{n-2}}\right)=0
$$

and

$$
\frac{1}{4}\left(f^{b}\right)^{\prime \prime \prime}-\delta\left(f^{b}\right)^{\prime \prime}+\left(\delta^{2}-\delta^{\prime}\right)\left(f^{b}\right)^{\prime}=-\frac{1}{2} \frac{x^{2}-\alpha^{2}}{\left(x^{2}+\alpha^{2}\right)^{3}} .
$$

Our current is built from the multiplier vector fields

$$
X^{a}=f^{a} \frac{\partial}{\partial r^{*}}, \quad X^{b}=f^{b} \frac{\partial}{\partial r^{*}}
$$

by setting

$$
J_{\mu}^{(\alpha)}(\phi) \doteq J_{\mu}^{X^{a}, 0}(\phi)+\sum_{i=1}^{\frac{n(n-1)}{2}} J_{\mu}^{X^{b}, 2}\left(\Omega_{i} \phi\right)
$$


and will be shown to have the property that its divergence

$$
K^{(\alpha)} \doteq \nabla^{\mu} J_{\mu}^{(\alpha)}
$$

is nonnegative upon integration over the spheres.

Proposition 4.17 (positivity of the current $J^{(\alpha)}$ ). For $n \geq 3$ and $\phi \in \mathrm{H}^{1}(S)$,

$$
\int_{S} K^{(\alpha)} \mathrm{d} \mu_{\gamma} \geq 0
$$

provided $\alpha$ is chosen sufficiently large, and $C(n, m, \alpha)$ set to be $(*)$ below.

Proof. In view of (4-62) and (4-63),

$$
\begin{aligned}
K^{(\alpha)} \geq & \frac{\left(f^{a}\right)^{\prime}}{1-\frac{2 m}{r^{n-2}}}\left(\frac{\partial \phi}{\partial r^{*}}\right)^{2}+\frac{f^{a}}{r}\left(1-\frac{n m}{r^{n-2}}\right)|\nabla \phi|_{r^{2} \dot{\gamma}_{n-1}}^{2}+\sum_{i=1}^{\frac{n(n-1)}{2}} \frac{f^{b}}{r}\left(1-\frac{n m}{r^{n-2}}\right)\left|\nabla \Omega_{i} \phi\right|_{r^{2} \dot{\gamma}_{n-1}}^{2} \\
& +\sum_{i=1}^{\frac{n(n-1)}{2}} F\left(\Omega_{i} \phi\right)^{2}+\sum_{i=1}^{\frac{n(n-1)}{2}} \frac{n-1}{4 r^{3}}\left[(n-3)+n \frac{2 m}{r^{n-2}}-(n-1)^{2}\left(\frac{2 m}{r^{n-2}}\right)^{2}\right] f^{b}\left(\Omega_{i} \phi\right)^{2},
\end{aligned}
$$

where

$$
F \doteq \frac{1}{2} \frac{1}{1-\frac{2 m}{r^{n-2}}} \frac{x^{2}-\alpha^{2}}{\left(x^{2}+\alpha^{2}\right)^{3}} .
$$

So, by Poincaré's inequality (4-50) and (4-51),

$$
\int_{S} K^{(\alpha)} \mathrm{d} \mu_{\gamma} \geq \int_{S}\left\{\frac{C(n-1)}{\alpha^{2} r^{n}}\left(\frac{\partial \phi}{\partial r^{*}}\right)^{2}+\left[(n-1) \frac{f^{b}}{r}\left(1-\frac{n m}{r^{n-2}}\right)+F r^{2}+\frac{1}{r} H\right]|\nabla \phi|_{r^{2} \dot{\gamma}_{n-1}}^{2}\right\} \mathrm{d} \mu_{\gamma},
$$

where

$$
H \doteq \frac{n-1}{4}\left[(n-3)+n \frac{2 m}{r^{n-2}}-(n-1)^{2}\left(\frac{2 m}{r^{n-2}}\right)^{2}\right] f^{b}-\frac{C}{\alpha^{2} r^{n-1}}\left(1-\frac{n m}{r^{n-2}}\right) .
$$

Step 1: $H \geq 0$. It is equivalent to show that

$$
\breve{H}(r) \doteq r^{n-1} H(r) \frac{r^{n-2}}{2 m}
$$

is nonnegative. We consider $\breve{H}$ to be a function of

so

$$
\rho \doteq \frac{r^{n-2}}{2 m}
$$

$$
\breve{H}=\frac{n-1}{4}(2 m r)\left[(n-3) \rho^{2}+n \rho-(n-1)^{2}\right] f^{b}-\frac{C}{\alpha^{2}}\left(\rho-\frac{n}{2}\right) \text {. }
$$

Note that

$$
r=\sqrt[n-2]{n m} \Longleftrightarrow \rho=\frac{n}{2} \Longleftrightarrow r^{*}=0
$$


and

$$
\breve{H}\left(\frac{n}{2}\right)=0 .
$$

Moreover we choose the constant $C$ such that

$$
\left.\frac{\mathrm{d} \breve{H}}{\mathrm{~d} \rho}\right|_{\rho=\frac{n}{2}}=0 .
$$

Then

$$
\begin{aligned}
\frac{\mathrm{d} \breve{H}}{\mathrm{~d} \rho}=\frac{n-1}{4}(2 m r)\left[\frac{(n-3)(2 n-3)}{n-2} \rho+\frac{n-1}{n-2} n-\frac{(n-1)^{2}}{n-2} \frac{1}{\rho}\right] f^{b} . \\
\quad+\frac{n-1}{4(n-2)} \frac{2 m r^{2}}{\rho-1}\left[(n-3) \rho^{2}+n \rho-(n-1)^{2}\right]\left(f^{b}\right)^{\prime}-\frac{C}{\alpha^{2}},
\end{aligned}
$$

where we have used

$$
\frac{\mathrm{d} r}{\mathrm{~d} \rho}=\frac{r}{(n-2) \rho}, \quad \frac{\mathrm{d} r^{*}}{\mathrm{~d} \rho}=\frac{1}{\rho-1} \frac{r}{n-2}
$$

Hence we choose

$$
C=\frac{(n-1)^{2}}{4(n-2)} \frac{\left(\frac{n}{2}\right)^{2}-(n-1)}{\frac{n}{2}-1} 2 m(n m)^{\frac{2}{n-2}} \frac{\alpha^{2}}{\alpha^{2}+(\alpha+\sqrt{\alpha})^{2}} .
$$

Note that then also

$$
\left.\frac{\mathrm{d} H}{\mathrm{~d} r}\right|_{r=\sqrt[n-2]{n m}}=0
$$

Now returning to the expression for $\breve{H}$, let us denote by $1 \leq \rho_{0} \leq \frac{n}{2}$ the value of $\rho$ for which

$$
(n-3) \rho_{0}+n-(n-1)^{2} \frac{1}{\rho_{0}}=0
$$

i.e.,

$$
\rho_{0}=\frac{2(n-1)^{2}}{n+\sqrt{n^{2}+4(n-1)^{2}(n-3)}} .
$$

We divide into the four regions

$$
1<\rho_{0}<\frac{n}{2}<\rho^{*}<\infty,
$$

where $\rho^{*}$ is to be chosen large enough below.

Step la (near the horizon, $1 \leq \rho \leq \rho_{0}$ ). Clearly $\breve{H} \geq 0$ termwise, because $f^{b} \leq 0$.

Step $1 b$ (near the photon sphere, $\rho_{0} \leq \rho \leq \frac{n}{2}$ ). We show $H=H(r)$ is convex on $r_{0} \leq r \leq \sqrt[n-2]{n m}$, where

$$
r_{0}=\sqrt[n-2]{\frac{4(n-1)^{2} m}{n+\sqrt{n^{2}+4(n-1)^{2}(n-3)}}} .
$$


Differentiating twice yields

$$
\begin{aligned}
\frac{\mathrm{d}^{2} H}{\mathrm{~d} r^{2}}= & \frac{n-1}{4} \frac{1}{\left(1-\frac{2 m}{r^{n-2}}\right)^{2}}\left(f^{b}\right)^{\prime \prime}\left[(n-3)+n \frac{2 m}{r^{n-2}}-(n-1)^{2}\left(\frac{2 m}{r^{n-2}}\right)^{2}\right] \\
& +\frac{n-1}{2} \frac{1}{1-\frac{2 m}{r^{n-2}}}\left(f^{b}\right)^{\prime}(n-2)\left[2(n-1)^{2} \frac{2 m}{r^{n-2}}-n\right] \frac{2 m}{r^{n-1}} \\
& -\frac{n-1}{4} \frac{1}{\left(1-\frac{2 m}{r^{n-2}}\right)^{2}}\left(f^{b}\right)^{\prime}(n-2)\left[(n-3)+n \frac{2 m}{r^{n-2}}-(n-1)^{2}\left(\frac{2 m}{r^{n-2}}\right)^{2}\right] \frac{2 m}{r^{n-1}} \\
& +\frac{n-1}{4}\left(f^{b}\right)\left[(n-2)(n-1) n \frac{2 m}{r^{n}}-2(2 n-3)(n-2)(n-1)^{2}\left(\frac{2 m}{r^{n-1}}\right)^{2}\right] \\
& -\frac{(n-1) n C}{\alpha^{n-1} r^{n+1}}\left(1-\frac{n m}{r^{n-2}}\right)+3 \frac{(n-1)(n-2) C}{\alpha^{n-1} r^{n}} \frac{n m}{r^{n-1}} .
\end{aligned}
$$

Since $\left(f^{b}\right)^{\prime \prime} \geq 0$, we further have in this region the bound

$$
\begin{aligned}
& \frac{\mathrm{d}^{2} H}{\mathrm{~d} r^{2}} \geq \frac{n-1}{2} \frac{1}{1-\frac{2 m}{r^{n-2}}} \times\left[2(n-1)^{2} \frac{2 m}{r^{n-2}}-n-\frac{1}{2} \frac{1}{1-\frac{2 m}{r_{0}^{n-2}}}\left((n-3)+n \frac{2 m}{r^{n-2}}-(n-1)^{2}\left(\frac{2 m}{r^{n-2}}\right)^{2}\right)\right] \\
& \times \frac{2 m}{r^{n-1}}(n-2)\left(f^{b}\right)^{\prime}+\frac{n-1}{4} \frac{2 m}{r^{n-2}}\left[1-\frac{2(2 n-3)(n-1)}{n}\left(\frac{2 m}{r^{n-2}}\right)\right] \frac{\left(f^{b}\right)}{r^{2}} .
\end{aligned}
$$

Since, for $n \geq 3$,

$$
\begin{gathered}
2(n-1)^{2} \frac{2}{n}-n-\frac{1}{2} \frac{2(n-1)^{2}}{2(n-1)^{2}-n-\sqrt{n^{2}+4(n-1)^{2}(n-3)}}\left((n-3)+2-\left(2 \frac{n-1}{n}\right)^{2}\right) \geq 1 \\
1-\frac{2(2 n-3)(n-1)}{n} \frac{2}{n} \leq-1,
\end{gathered}
$$

we finally obtain in this region

$$
\frac{\mathrm{d}^{2} H}{\mathrm{~d} r^{2}} \geq \frac{(n-1)(n-2)}{2 r} \frac{1}{\rho-1}\left(f^{b}\right)^{\prime}>0 .
$$

Step 1c (in the intermediate region, $\frac{n}{2} \leq \rho \leq \rho^{*}$ ). We show $\breve{H}=\breve{H}(\rho)$ is convex on $\frac{n}{2} \leq \rho \leq \rho^{*}$ for $r^{*}\left(\rho=\rho^{*}\right) \leq \alpha$. We have

$$
\begin{aligned}
\frac{\mathrm{d}^{2} \breve{H}}{\mathrm{~d} \rho^{2}}= & \frac{(n-1)^{2}}{4(n-2)^{2}} \frac{2 m r}{\rho^{2}}\left[(n-3)(2 n-3) \rho^{2}+n \rho+(n-3)(n-1)\right]\left(f^{b}\right) \\
& +\frac{(n-1)^{2}}{4(n-2)^{2}} \frac{2 m r^{2}}{(\rho-1)^{2}}\left[3(n-3) \rho^{2}-3(n-5) \rho+(n-1)(n-5)-n \frac{2 n-1}{n-1}+3(n-1) \frac{1}{\rho}\right]\left(f^{b}\right)^{\prime} \\
& +\frac{n-1}{4(n-2)^{2}} \frac{2 m r^{3}}{(\rho-1)^{2}}\left[(n-3) \rho^{2}+n \rho-(n-1)^{2}\right]\left(f^{b}\right)^{\prime \prime}
\end{aligned}
$$


Since, for $\rho \geq \frac{n}{2}$ and $n \geq 3$,

$3(n-3) \rho(\rho-1)+6 \rho+(n-1)(n-5)-n \frac{2 n-1}{n-1}+3(n-1) \frac{1}{\rho} \geq 1 \quad$ and $\quad(n-3) \rho^{2}+n \rho-(n-1)^{2} \geq 0$, we have

$$
\frac{\mathrm{d}^{2} \breve{H}}{\mathrm{~d} \rho^{2}} \geq \frac{(n-1)^{2}}{4(n-2)^{2}} \frac{2 m r^{2}}{(\rho-1)^{2}}\left(f^{b}\right)^{\prime}>0,
$$

because $\left(f^{b}\right) \geq 0$ for $r^{*} \geq 0$, and $\left(f^{b}\right)^{\prime \prime} \geq 0$ for $x \leq 0$.

Step $1 d$ (in the asymptotics, $\left.\rho \geq \rho^{*}\right)$. We show directly $H(r)>0$ for $r^{*} \geq R^{*} \doteq r^{*}\left(\rho=\rho^{*}\right)$ and $\rho^{*}$ chosen large enough. Let $r^{*} \geq R^{*}, R^{*} \leq \alpha$. Then

$$
f^{b} \geq \int_{0}^{R^{*}}\left(f^{b}\right)^{\prime} \mathrm{d} r^{*}=\frac{1}{\alpha} \int_{-\left(1+\frac{1}{\sqrt{\alpha}}\right)}^{\frac{R^{*}-\alpha-\sqrt{\alpha}}{\alpha}} \frac{1}{1+t^{* 2}} \mathrm{~d} t^{*} \geq \frac{R^{*}}{5 \alpha^{2}}
$$

provided $\alpha \geq 1$, and of course

Thus

$$
f^{b} \leq\left.\frac{1}{\alpha} \arctan t^{*}\right|_{-\left(1+\frac{1}{\sqrt{\alpha}}\right)} ^{0} \leq \frac{\pi}{2 \alpha}
$$

$$
\begin{aligned}
H & =\frac{(n-1)(n-3)}{4}+\left[\frac{(n-1) n}{4} f^{b}-\frac{C}{\alpha^{2} 2 m} \frac{1}{r}\right] \frac{2 m}{r^{n-2}}-\left[\frac{(n-1)^{3}}{4} f^{b}-\frac{C n}{\alpha^{2} 4 m} \frac{1}{r}\right]\left(\frac{2 m}{r^{n-2}}\right)^{2} \\
& \geq \frac{1}{\alpha^{2}}\left[\frac{(n-1) n}{4} \frac{R^{*}}{5}-\frac{C}{2 m} \frac{1}{r}\right] \frac{2 m}{r^{n-2}}-\frac{(n-1)^{3}}{4} \frac{\pi}{2 \alpha}\left(\frac{2 m}{r^{n-2}}\right)^{2}>0
\end{aligned}
$$

for $R^{*}$ (and consequently $\alpha$ ) chosen large enough.

Step 2: (4-72). Since $\left(1-\frac{n m}{r^{n-2}}\right) f^{b} \geq 0$ and $F \geq 0$ for $|x| \geq \alpha$, we need to show

$$
(n-1)\left(f^{b}\right)\left(1-\frac{n m}{r^{n-2}}\right)+F r^{3} \geq 0
$$

for

$$
-\alpha \leq x \leq \alpha \Longleftrightarrow \sqrt{\alpha} \leq r^{*} \leq \sqrt{\alpha}+2 \alpha .
$$

In this whole region, in view of Proposition B.1,

$$
\lim _{\alpha \rightarrow \infty} \frac{r^{*}}{r}=1, \quad \lim _{\alpha \rightarrow \infty}\left(1-\frac{2 m}{r^{n-2}}\right)=\lim _{\alpha \rightarrow \infty}\left(1-\frac{n m}{r^{n-2}}\right)=1 .
$$

$n \geq 4:$ Since

$$
f^{b}\left(r^{*}\right) \geq \int_{\sqrt{\alpha}}^{r^{*}} \frac{1}{\alpha^{2}+x^{2}} \mathrm{~d} r^{*} \geq \frac{x+\alpha}{2 \alpha^{2}},
$$

it suffices to show

$$
(n-1) \frac{x+\alpha}{2 \alpha^{2}}+\frac{1}{2} \frac{x^{2}-\alpha^{2}}{\left(x^{2}+\alpha^{2}\right)^{3}} r^{3} \geq 0,
$$


which is implied by

$$
\frac{\alpha-x}{n-1} \frac{(x+\alpha+\sqrt{\alpha})^{3}}{\left(x^{2}+\alpha^{2}\right)^{2}} \leq 1
$$

For $-\alpha \leq x \leq 0$,

$$
(x+\alpha+\sqrt{\alpha})^{3} \leq \alpha^{3}\left(1+\frac{1}{\sqrt{\alpha}}\right)^{3} \leq \frac{4}{3} \alpha^{3}
$$

for $\alpha$ large enough; thus

$$
\frac{\alpha-x}{n-1} \frac{(x+\alpha+\sqrt{\alpha})^{3}}{\left(x^{2}+\alpha^{2}\right)^{2}} \leq \frac{1}{n-1} \frac{2 \alpha}{\alpha^{4}} \frac{4}{3} \alpha^{3} \leq \frac{8}{9} .
$$

For $0 \leq x \leq \alpha$, we have to show

$$
\frac{\alpha}{n-1} \frac{(x+\alpha+\sqrt{\alpha})^{3}}{\left(x^{2}+\alpha^{2}\right)^{2}} \leq 1
$$

Since

$$
(x+\alpha+\sqrt{\alpha})^{3} \leq 2^{\frac{3}{2}}\left(1+\frac{1}{\sqrt{\alpha}}\right)^{3}\left(x^{2}+\alpha^{2}\right)^{\frac{3}{2}},
$$

we have, for $\alpha$ large enough,

$$
\frac{\alpha}{n-1} \frac{(x+\alpha+\sqrt{\alpha})^{3}}{\left(x^{2}+\alpha^{2}\right)^{2}} \leq \frac{\alpha}{n-1} \frac{2^{\frac{3}{2}}\left(1+\frac{1}{\sqrt{\alpha}}\right)^{3}}{\left(x^{2}+\alpha^{2}\right)^{\frac{1}{2}}} \leq \frac{2^{\frac{3}{2}}}{3}\left(1+\frac{1}{\sqrt{\alpha}}\right)^{3}<1 .
$$

$n=3$ : We see that (4-76) and (4-77) fail in the case $n=3$, as a consequence of which also (4-75) fails to hold. In the case $n=3$, we have to use a better approximation of (4-73); see [Dafermos and Rodnianski 2007] for details. Note also that in view of (4-75), the positivity property (4-72) is "easily" satisfied for large values of $n$, which indicates that there may be yet another simplified proof in higher dimensions.

Given the strict inequalities proven in Step 2 of the proof of Proposition 4.17, for $\alpha$ chosen large enough, we can keep a fraction of the manifestly nonnegative $\left|\not \nabla \Omega_{i} \phi\right|^{2}$ term in (4-67). Furthermore we have obtained control on the $|\not \phi|^{2}$ term from (4-69).

Corollary 4.18. Let $\phi \in \mathrm{H}^{2}(S)$ be a solution of the wave equation (1-1). Then there exists a constant $C(n, m)$ and a current $K$ such that

$$
\begin{array}{r}
\int_{S}\left\{\frac{1}{r^{n}}\left(\frac{\partial \phi}{\partial r^{*}}\right)^{2}+\frac{1}{r^{n+1}}\left(\frac{\partial \phi}{\partial t}\right)^{2}+r\left(1-\frac{n m}{r^{n-2}}\right)^{2}\left|\not \nabla^{2} \phi\right|_{r^{2} \dot{\gamma}_{n-1}}^{2}+\frac{r^{2}}{\left(1-\frac{2 m}{r^{n-2}}\right)\left(1+r^{* 2}\right)^{2}}|\not \phi \phi|_{r^{2} \dot{\gamma}_{n-1}}^{2}\right\} \mathrm{d} \mu_{\gamma} \\
\leq C(n, m) \int_{S} K \mathrm{~d} \mu_{\gamma} .
\end{array}
$$

Proof. Set $K=K^{(\alpha)}+K^{\text {aux }}$ and choose $\alpha$ large enough.

Here we retrieve the time derivatives with the auxiliary current

$$
K^{\text {aux }}=\nabla^{\mu} J_{\mu}^{\text {aux }} ; \quad J^{\text {aux }}=J^{X^{\text {aux }}, 0} ; \quad X^{\text {aux }}=f^{\text {aux }} \frac{\partial}{\partial r^{*}},
$$


where $f^{\text {aux }}=-\frac{1}{r^{n}}$ satisfies

for, in view of (4-9),

$$
\left(f^{\text {aux }}\right)^{\prime}+(n-1) \frac{f^{\text {aux }}}{r}\left(1-\frac{2 m}{r^{n-2}}\right)=\frac{1}{r^{n+1}}\left(1-\frac{2 m}{r^{n-2}}\right)
$$

$$
\frac{1}{r^{n+1}}\left(\frac{\partial \phi}{\partial t}\right)^{2} \leq 2 K^{\mathrm{aux}}+3 \frac{1}{r^{n+1}}|\not \nabla \phi|_{r^{2} \dot{\gamma}_{n-1}}^{2}
$$

4D. Boundary terms. In this section we first prove Proposition 4.1 and then a refinement thereof for finite regions, which requires us to estimate the boundary terms of the currents introduced in Sections 4B and $4 \mathrm{C}$.

4D.1. Proof of Proposition 4.1. We can now combine our earlier results Corollary 4.14 and Corollary 4.18 to prove the integrated local energy decay estimate (4-4); note that there is no restriction on the spherical harmonic number, and that no commutation with angular momentum operators is required.

Proof of Proposition 4.1. Write

$$
\phi=\pi_{<L} \phi+\pi_{\geq L} \phi
$$

with

$$
\pi_{<L}=\sum_{l=0}^{L-1} \pi_{l} \phi, \quad \pi_{\geq L}=\sum_{l=L}^{\infty} \pi_{l} \phi,
$$

where $L=(6 \gamma n)^{2}$ is fixed (recall here $\gamma=12$ from Section 4B).

Step 1 (high spherical harmonics). By Corollary 4.14,

$$
\int_{\mathcal{R}\left(t_{0}, t_{1}, u_{1}^{*}, v_{1}^{*}\right)} \frac{1}{4} \frac{1}{8} \frac{(2 m)^{\frac{3}{n-2}}}{r^{3}}\left(\pi_{\geq L} \phi\right)^{2} \leq \int_{\mathcal{R}\left(t_{0}, t_{1}, u_{1}^{*}, v_{1}^{*}\right)} K^{X_{\gamma, \alpha}, 1}\left(\pi_{\geq L} \phi\right) .
$$

It remains to estimate the boundary terms of the current $J^{X_{\gamma, \alpha}, 1}$, and to use this estimate to recover all derivatives using a commutation with the Killing vector field $T$.

Step 1a (boundary terms). We may assume $\left|r_{0,1}^{*}\right| \geq \frac{4}{\gamma \alpha}, r_{0,1}$ entering the definition (4-3). Recalling the properties of $f_{\gamma, \alpha}$ away from the photon sphere, we find

$$
\begin{aligned}
& \left|\left(J^{X_{\gamma, \alpha}, 1}\left(\pi_{\geq L} \phi\right), \frac{\partial}{\partial v^{*}}\right)\right| \\
& \leq\left|\left(J^{X_{\gamma, \alpha}}\left(\pi_{\geq L} \phi\right), \frac{\partial}{\partial v^{*}}\right)\right|+\frac{1}{2}\left|f_{\gamma, \alpha}{ }^{\prime}+(n-1) \frac{f_{\gamma, \alpha}}{r}\left(1-\frac{2 m}{r^{n-2}}\right)\right|\left(\pi_{\geq L} \phi\right)\left(\frac{\partial \pi_{\geq L}}{\partial v^{*}}\right) \\
& +\frac{1}{4}\left|\left(f_{\gamma, \alpha}{ }^{\prime}+(n-1) \frac{f_{\gamma, \alpha}}{r}\left(1-\frac{2 m}{r^{n-2}}\right)\right)^{\prime}\right|\left(\pi_{\geq L} \phi\right)^{2} \\
& \leq \frac{n+1}{(\gamma \alpha)^{3}}\left(J^{T}\left(\pi_{\geq L} \phi\right), \frac{\partial}{\partial v^{*}}\right)+\frac{1}{(\gamma \alpha)^{6}} \frac{1}{\left|r^{*}\right|^{4}}\left(\frac{\partial \pi_{\geq L} \phi}{\partial v^{*}}\right)^{2}+\frac{1}{(\gamma \alpha)^{6}} \frac{1}{\left|r^{*}\right|^{4}}\left[1+\frac{1}{\left|r^{*}\right|}\right]\left(\pi_{\geq L} \phi\right)^{2} \\
& +\frac{n-1}{(\gamma \alpha)^{3}}\left[n+\frac{4}{(\gamma \alpha)^{6}} \frac{r}{\left|r^{*}\right|^{4}}\right] \frac{1}{2 r^{2}}\left(1-\frac{2 m}{r^{n-2}}\right)\left(\pi_{\geq L} \phi\right)^{2},
\end{aligned}
$$


and, by Lemma 4.6,

similarly for

$$
\int_{S_{r}} \frac{1}{2} \frac{1}{r^{2}}\left(1-\frac{2 m}{r^{n-2}}\right)\left(\pi_{\geq L} \phi\right)^{2} \leq \frac{1}{(6 \gamma n)^{4}} \int_{S_{r}}\left(J^{T}, \frac{\partial}{\partial v^{*}}\right)
$$

$$
\left|\left(J^{X_{\gamma, \alpha}, 1}\left(\pi_{\geq L} \phi\right), \frac{\partial}{\partial u^{*}}\right)\right| .
$$

Since also, by Lemma C.7 and Lemma 4.6,

$$
\begin{aligned}
& \left.\int_{\frac{1}{2}\left(t_{0}-r_{0}^{*}\right)}^{u_{1}^{*}} \mathrm{~d} u^{*} \int_{\mathbb{S}^{n-1}} \mathrm{~d} \mu_{\dot{\gamma}_{n-1}} r^{n-1} \frac{1}{r^{* 4}}\left(\pi_{\geq L} \phi\right)^{2}\right|_{v^{*}=\frac{1}{2}\left(t_{0}+r_{0}^{*}\right)} \\
& \leq \frac{8}{\left|r_{0}^{*}\right|^{4}} \frac{\left(1+\left|r_{0}^{*}\right|^{2}\right)^{2}}{\left|r_{0}^{*}\right|^{2}} \int_{\frac{1}{2}\left(t_{0}-r_{0}^{*}\right)}^{\infty} \int_{\mathbb{S}^{n-1}} \mathrm{~d} \mu_{\dot{\gamma}_{n-1}} r^{n-1}\left(\frac{\partial \pi_{\geq L} \phi}{\partial u^{*}}\right)^{2} \mathrm{~d} u^{*} \\
& \quad+2 \pi \frac{1+\left|r_{0}^{*}\right|^{2}}{\left|r_{0}^{*}\right|^{4}}\left[1+\frac{(n m)^{\frac{2}{n-2}}}{(6 \gamma n)^{4}}\right] \int_{\frac{1}{2}\left(t_{0}-r_{0}^{*}\right)}^{\frac{1}{2}\left(t_{0}-r_{0}^{*}\right)+1} \int_{\mathbb{S}^{n-1}} \mathrm{~d} \mu_{\dot{\gamma}_{n-1}} r^{n-1}\left\{\left|\nabla \pi_{\geq L} \phi\right|^{2}+\left(\frac{\partial \pi_{\geq L} \phi}{\partial u^{*}}\right)^{2}\right\} \mathrm{d} u^{*},
\end{aligned}
$$

there is a constant $C(n, m)$ (recall $\left.\gamma=12, \alpha=(n-1) /(n m)^{\frac{1}{n-2}}\right)$ such that

$$
\begin{aligned}
\int_{\frac{1}{2}\left(t_{0}-r_{0}^{*}\right)}^{u_{1}^{*}} \mathrm{~d} u^{*} \int_{\mathbb{S}^{n-1}} \mathrm{~d} \mu_{\dot{\gamma}_{n-1}} r^{n-1}\left|\left(J^{X_{\gamma, \alpha}, 1}\left(\pi_{\geq L} \phi\right), \frac{\partial}{\partial u^{*}}\right)\right| \\
\leq\left. C(n, m) \int_{\frac{1}{2}\left(t_{0}-r_{0}^{*}\right)}^{\infty} \mathrm{d} u^{*} \int_{\mathbb{S}^{n-1}} \mathrm{~d} \mu_{\dot{\gamma}_{n-1}} r^{n-1}\left(J^{T}\left(\pi_{\geq L} \phi\right), \frac{\partial}{\partial u^{*}}\right)\right|_{v^{*}=\frac{1}{2}\left(t_{0}+r_{0}^{*}\right)} .
\end{aligned}
$$

To establish

$$
\int_{\mathbb{S}^{n-1}}\left|\left(J^{X_{\gamma, \alpha}, 1}\left(\pi_{\geq L} \phi\right), \frac{\partial}{\partial t}\right)\right| r^{n-1} \mathrm{~d} \mu_{\dot{\gamma}_{n-1}} \leq C(n, m) \int_{\mathbb{S}^{n-1}}\left(J^{T}\left(\pi_{\geq L} \phi\right), \frac{\partial}{\partial t}\right) r^{n-1} \mathrm{~d} \mu_{\dot{\gamma}_{n-1}},
$$

note that

$$
\begin{aligned}
\left|\left(J^{X_{\gamma, \alpha}, 1}\left(\pi_{\geq L} \phi\right), \frac{\partial}{\partial t}\right)\right| \leq & \left|\left(J^{X_{\gamma, \alpha}}\left(\pi_{\geq L} \phi\right), \frac{\partial}{\partial t}\right)\right|+\frac{1}{2}\left|f_{\gamma, \alpha}{ }^{\prime}+(n-1) \frac{f_{\gamma, \alpha}}{r}\left(1-\frac{2 m}{r^{n-2}}\right)\right|\left(\pi_{\geq L} \phi\right)\left(\frac{\partial \pi_{\geq L} \phi}{\partial t}\right) \\
\leq & \left|f_{\gamma, \alpha}\right|\left|T\left(\pi_{\geq L} \phi\right)\left(\frac{\partial}{\partial r^{*}}, \frac{\partial}{\partial t}\right)\right| \\
& +\frac{1}{2}\left[\frac{1}{2} r^{2}\left|f_{\gamma, \alpha}{ }^{\prime}\right|+\frac{3}{2} \frac{n-1}{2} \frac{1}{(\gamma \alpha)^{3}}\left(1-\frac{2 m}{r^{n-2}}\right)^{2}\right] \frac{1}{r^{2}}\left(\pi_{\geq L} \phi\right)^{2} \\
& +\frac{1}{2} \frac{1}{(\gamma \alpha)^{2}}\left[1+\frac{3}{2} \frac{n-1}{2} \frac{1}{\gamma \alpha}\right]\left(\frac{\partial \pi_{\geq L} \phi}{\partial t}\right)^{2},
\end{aligned}
$$

and, by Lemma 4.6,

$$
\int_{S_{r}} \frac{1}{2} \frac{1}{r^{2}}\left(\pi_{\geq L} \phi\right)^{2} \mathrm{~d} \mu_{\gamma} \leq \frac{1}{(6 \gamma n)^{4}}\left(1-\frac{2 m}{r_{0}^{n-2}}\right)^{-1} \int_{S_{r}}\left(J^{T}\left(\pi_{\geq L} \phi\right), \frac{\partial}{\partial t}\right) \mathrm{d} \mu_{\gamma},
$$


which suffices in view of the properties of $f_{\gamma, \alpha}$, in particular that there is a constant $r^{2}\left|f_{\gamma, \alpha}\right| \leq C(n, m)$. For the boundary term

$$
\begin{aligned}
\int_{\mathbb{S}^{n-1}}\left|\left(J^{X_{\gamma, \alpha}, 1}\left(\pi_{\geq L} \phi\right), \frac{\partial}{\partial v^{*}}\right)\right|_{u^{*}=u_{1}^{*}} r^{n-1} \mathrm{~d} \mu_{\gamma_{n-1}} & \\
\leq \int_{\mathbb{S}^{n-1}}\left\{\frac { 1 } { ( \gamma \alpha ) ^ { 3 } } \left[n+1+\frac{1}{2} \frac{1}{(\gamma \alpha)^{2}}\right.\right. & \left.+\frac{n-1}{(6 \gamma n)^{4}}\left(n+\frac{4(n m)^{\frac{1}{n-2}}}{(\gamma \alpha)^{2}}\right)\right]\left(J^{T}\left(\pi_{\geq L} \phi\right), \frac{\partial}{\partial v^{*}}\right) \\
& \left.\quad+\frac{1}{(\gamma \alpha)^{6}} \frac{1}{\left|r^{*}\right|^{4}}\left(1+\frac{\gamma \alpha}{4}\right)\left(\pi_{\geq L} \phi\right)^{2}\right\} r^{n-1} \mathrm{~d} \mu_{\dot{\gamma}_{n-1}},
\end{aligned}
$$

we find (using the boundedness of $\phi$ on the horizon; see Section 5A) in the limit $u_{1}^{*} \rightarrow \infty$ a constant $C(n, m)$ such that

$\left.\int_{\mathbb{S}^{n-1}}\left|\left(J^{X_{\gamma \alpha}, 1}\left(\pi_{\geq L} \phi\right), \frac{\partial}{\partial v^{*}}\right)\right| r^{n-1} \mathrm{~d} \mu_{\dot{\gamma}_{n-1}}\right|_{u^{*}=\infty} \leq\left. C(n, m) \int_{\mathbb{S}^{n-1}}\left(J^{T}\left(\pi_{\geq L} \phi\right), \frac{\partial}{\partial v^{*}}\right) r^{n-1} \mathrm{~d} \mu_{\dot{\gamma}_{n-1}}\right|_{u^{*}=\infty}$.

We conclude that there is a constant $C(n, m)$ such that

$$
\int_{\mathcal{R}_{r_{0}, r_{1}}^{\infty}\left(t_{0}\right)} \frac{(2 m)^{\frac{3}{n-2}}}{r^{3}}\left(\pi_{\geq L} \phi\right)^{2} \leq C(n, m) \int_{\Sigma_{\tau_{0}}}\left(J^{T}\left(\pi_{\geq L} \phi\right), n\right),
$$

where $\tau_{0}=\frac{1}{2}\left(t_{0}-r_{1}^{*}\right)$ because

$$
\square_{g}\left(\pi_{\geq L} \phi\right)=0, \quad K^{T}\left(\pi_{\geq L} \phi\right)=0 .
$$

Step $1 b$ (commutation with $T$ ). Since

$$
\square_{g}\left(T \cdot \pi_{\geq L} \phi\right)=0,
$$

we also have

$$
\int_{\mathcal{R}_{r_{0}, r_{1}}^{\infty}\left(t_{0}\right)} \frac{(2 m)^{\frac{3}{n-2}}}{r^{3}}\left(\frac{\partial \pi_{\geq L} \phi}{\partial t}\right)^{2} \leq C(n, m) \int_{\Sigma_{\tau_{0}}}\left(J^{T}\left(T \cdot \pi_{\geq L} \phi\right), n\right) .
$$

This is enough to control the remaining derivatives, too; for the auxiliary current (C-10) yields

$$
K^{\text {aux }}=\phi\left(\partial^{\mu} h\right)\left(\partial_{\mu} \phi\right)+h \partial^{\alpha} \phi \partial_{\alpha} \phi,
$$

which, upon choosing

$$
h=\left(1-\frac{2 m}{r^{n-2}}\right) \frac{(2 m)^{\frac{3}{n-2}}}{r^{3}},
$$

presents us with

$$
K^{\text {aux }}=\phi \frac{\partial h}{\partial r} \frac{\partial \phi}{\partial r^{*}}-\frac{(2 m)^{\frac{3}{n-2}}}{r^{3}}\left(\frac{\partial \phi}{\partial t}\right)^{2}+\frac{(2 m)^{\frac{3}{n-2}}}{r^{3}}\left(\frac{\partial \phi}{\partial r^{*}}\right)^{2}+\frac{(2 m)^{\frac{3}{n-2}}}{r^{3}}\left(1-\frac{2 m}{r^{n-2}}\right)|\not \nabla \phi|_{r^{2} \hat{\gamma}_{n-1}}^{2} .
$$


Using Cauchy's inequality for the first term, namely

$$
\begin{aligned}
\phi \frac{\partial h}{\partial r} \frac{\partial \phi}{\partial r^{*}} & =(n-2) \phi \frac{2 m}{r^{n-1}} \frac{(2 m)^{\frac{3}{n-2}}}{r^{3}} \frac{\partial \phi}{\partial r^{*}}-3\left(1-\frac{2 m}{r^{n-2}}\right) \phi \frac{(2 m)^{\frac{3}{n-2}}}{r^{4}} \frac{\partial \phi}{\partial r^{*}} \\
& \geq-\frac{1}{2} \frac{(2 m)^{\frac{3}{n-2}}}{r^{3}}\left(\frac{\partial \phi}{\partial r^{*}}\right)^{2}-\left(\frac{n-2}{r}\right)^{2}\left(\frac{2 m}{r^{n-2}}\right)^{2} \frac{(2 m)^{\frac{3}{n-2}}}{r^{3}} \phi^{2}-\left(\frac{3}{r}\right)^{2}\left(1-\frac{2 m}{r^{n-2}}\right)^{2} \frac{(2 m)^{\frac{3}{n-2}}}{r^{3}} \phi^{2} \\
& \geq-\frac{1}{2} \frac{(2 m)^{\frac{3}{n-2}}}{r^{3}}\left(\frac{\partial \phi}{\partial r^{*}}\right)^{2}-2 \frac{n^{2}}{(2 m)^{\frac{2}{n-2}}} \frac{(2 m)^{\frac{3}{n-2}}}{r^{3}} \phi^{2}
\end{aligned}
$$

we obtain the bound

$$
\begin{aligned}
K^{\text {aux }} \geq \frac{1}{2} \frac{(2 m)^{\frac{3}{n-2}}}{r^{3}}\left(\frac{\partial \phi}{\partial r^{*}}\right)^{2}+\frac{(2 m)^{\frac{3}{n-2}}}{r^{3}}\left(1-\frac{2 m}{r^{n-2}}\right)|\not \phi|_{r^{2} \dot{\gamma}_{n-1}}^{2} & -\frac{(2 m)^{\frac{3}{n-2}}}{r^{3}}\left(\frac{\partial \phi}{\partial t}\right)^{2}-2 \frac{n^{2}}{(2 m)^{\frac{2}{n-2}}} \frac{(2 m)^{\frac{3}{n-2}}}{r^{3}} \phi^{2} .
\end{aligned}
$$

Therefore

$$
\begin{aligned}
\int_{\mathcal{R}_{r_{0}, r_{1}}^{\infty}\left(t_{0}\right)} & \left\{\frac{1}{2} \frac{(2 m)^{\frac{3}{n-2}}}{r^{3}}\left(\frac{\partial \pi_{\geq L} \phi}{\partial r^{*}}\right)^{2}+\frac{(2 m)^{\frac{3}{n-2}}}{r^{3}}\left(1-\frac{2 m}{r^{n-2}}\right)\left|\not \pi_{\geq L} \phi\right|_{r^{2}{ }^{\prime} n-1}^{2}\right\} \\
\leq & \int_{\mathcal{R}_{r_{0}, r_{1}}^{\infty}\left(t_{0}\right)}\left\{K^{\mathrm{aux}}\left(\pi_{\geq L} \phi\right)+\frac{(2 m)^{\frac{3}{n-2}}}{r^{3}}\left(\frac{\partial \pi_{\geq L} \phi}{\partial t}\right)^{2}+2 \frac{n^{2}}{(2 m)^{\frac{2}{n-2}}} \frac{(2 m)^{\frac{3}{n-2}}}{r^{3}}\left(\pi_{\geq L} \phi\right)^{2}\right\} .
\end{aligned}
$$

The boundary terms are controlled using Proposition C.8:

$$
\int_{\mathcal{R}_{r_{0}, r_{1}}^{\infty}\left(t_{0}\right)} K^{\mathrm{aux}}\left(\pi_{\geq L} \phi\right) \leq C(n, m) \int_{\Sigma_{\tau_{0}}}\left(J^{T}\left(\pi_{\geq L} \phi\right), n\right) .
$$

Hence

$$
\begin{aligned}
& \int_{\mathcal{R}_{r_{0}, r_{1}}^{\infty}\left(t_{0}\right)} \frac{(2 m)^{\frac{3}{n-2}}}{r^{3}}\left\{\left(\frac{\partial \pi_{\geq L} \phi}{\partial r^{*}}\right)^{2}+\left(1-\frac{2 m}{r^{n-2}}\right)\left|\nabla \pi_{\geq L} \phi\right|_{r^{2} \dot{\gamma}_{n-1}}^{2}\right\} \\
& \leq C(n, m) \int_{\Sigma_{\tau_{0}}}\left(J^{T}\left(\pi_{\geq L} \phi\right)+J^{T}\left(T \cdot \pi_{\geq L} \phi\right), n\right) .
\end{aligned}
$$

Step 2 (low spherical harmonics). Now recall the $J^{(\alpha)}$ current (4-65); we will show in a first step that

$$
\int_{\mathcal{R}_{r_{0}, r_{1}}^{\infty}\left(t_{0}\right)} K^{(\alpha)}(\phi) \leq C(n, m) \int_{\Sigma_{\tau_{0}}}\left(J^{T}(\phi)+\sum_{i=1}^{\frac{n(n-1)}{2}} J^{T}\left(\Omega_{i} \phi\right), n\right) .
$$

Then in particular, by Corollary 4.18, 


$$
\begin{aligned}
\int_{\mathcal{R}_{r_{0}, r_{1}}^{\infty}\left(t_{0}\right)}\left\{\frac{1}{r^{n}}\left(\frac{\partial \pi_{<L} \phi}{\partial r^{*}}\right)^{2}+\frac{1}{r^{n+1}}\right. & \left.\left(\frac{\partial \pi_{<L} \phi}{\partial t}\right)^{2}+\frac{r^{2}}{\left(1-\frac{2 m}{r^{n-2}}\right)\left(1+\left|r^{*}\right|^{2}\right)^{2}}\left|\not \nabla \pi_{<L} \phi\right|_{r^{2} \hat{\gamma}_{n-1}}^{2}\right\} \\
& \leq C(n, m) \int_{\Sigma_{\tau_{0}}}\left(J^{T}\left(\pi_{<L} \phi\right)+\sum_{i=1}^{\frac{n(n-1)}{2}} J^{T}\left(\Omega_{i} \cdot \pi_{<L} \phi\right), n\right) .
\end{aligned}
$$

But in a second step we will show that in fact there exists a constant $C(n)$ such that

$$
\int_{\Sigma_{\tau_{0}}} \sum_{i=1}^{\frac{n(n-1)}{2}}\left(J^{T}\left(\Omega_{i} \cdot \pi_{<L} \phi\right), n\right) \leq C(n) \int_{\Sigma_{\tau_{0}}}\left(J^{T}\left(\pi_{<L} \phi\right), n\right) .
$$

Step $2 a$ (boundary terms). The energy identity for $J^{(\alpha)}$ on the domain (4-1) implies, more explicitly,

$$
\begin{aligned}
& \int_{\mathcal{R}\left(t_{0}, t_{1}, u_{1}^{*}, v_{1}^{*}\right)} K^{(\alpha)} \leq\left.\int_{\frac{1}{2}\left(t_{0}+r_{0}^{*}\right)}^{\frac{1}{2}\left(t_{1}+r_{0}^{*}\right)} \int_{\mathbb{S}^{n-1}}\left|\left(J^{(\alpha)}, \frac{\partial}{\partial v^{*}}\right)\right| r^{n-1}\right|_{u^{*}=u_{1}^{*}} \mathrm{~d} v^{*} \mathrm{~d} \mu_{\dot{\gamma}_{n-1}} \\
&+\left.\int_{\frac{1}{2}\left(t_{1}-r_{0}^{*}\right)}^{u_{1}^{*}} \int_{\mathbb{S}^{n-1}}\left|\left(J^{(\alpha)}, \frac{\partial}{\partial u^{*}}\right)\right| r^{n-1}\right|_{v^{*}=\frac{1}{2}\left(t_{1}+r_{0}^{*}\right)} \mathrm{d} u^{*} \mathrm{~d} \mu_{\dot{\gamma}_{n-1}} \\
&+\left.\int_{r_{0}^{*}}^{r_{1}^{*}} \int_{\mathbb{S}^{n-1}}\left|\left(J^{(\alpha)}, T\right)\right| r^{n-1}\right|_{t=t_{1}} \mathrm{~d} r^{*} \mathrm{~d} \mu_{\dot{\gamma}_{n-1}} \\
&+\left.\int_{\frac{1}{2}\left(t_{1}+r_{1}^{*}\right)}^{v_{1}^{*}} \int_{\mathbb{S}^{n-1}}\left|\left(J^{(\alpha)}, \frac{\partial}{\partial v^{*}}\right)\right| r^{n-1}\right|_{u^{*}=\frac{1}{2}\left(t_{1}-r_{1}^{*}\right)} \mathrm{d} v^{*} \mathrm{~d} \mu_{\dot{\gamma}_{n-1}} \\
&+\left.\int_{\frac{1}{2}\left(t_{0}-r_{1}^{*}\right)}^{\frac{1}{2}\left(t_{1}-r_{1}^{*}\right)} \int_{\mathbb{S}^{n-1}}\left|\left(J^{(\alpha)}, \frac{\partial}{\partial u^{*}}\right)\right| r^{n-1}\right|_{v^{*}=v_{1}^{*}} \mathrm{~d} v^{*} \mathrm{~d} \mu_{\dot{\gamma}_{n-1}} \\
&+\left.\int_{\frac{1}{2}\left(t_{0}-r_{0}^{*}\right)}^{u_{1}^{*}} \int_{\mathbb{S}^{n-1}}\left|\left(J^{(\alpha)}, \frac{\partial}{\partial u^{*}}\right)\right| r^{n-1}\right|_{v^{*}=\frac{1}{2}\left(t_{0}+r_{0}^{*}\right)} \mathrm{d} u^{*} \mathrm{~d} \mu_{\dot{\gamma}_{n-1}} \\
&+\left.\int_{r_{0}^{*}}^{r_{1}^{*}} \int_{\mathbb{S}^{n-1}}\left|\left(J^{(\alpha)}, T\right)\right| r^{n-1}\right|_{t=t_{0}} \mathrm{~d} r^{*} \mathrm{~d} \mu_{\dot{\gamma}_{n-1}} \\
&+\left.\int_{\frac{1}{2}\left(t_{0}+r_{1}^{*}\right)}^{v_{1}^{*}} \int_{\mathbb{S}^{n-1}}\left|\left(J^{(\alpha)}, \frac{\partial}{\partial v^{*}}\right)\right| r^{n-1}\right|_{u^{*}=\frac{1}{2}\left(t_{0}-r_{1}^{*}\right)} \mathrm{d} v^{*} \mathrm{~d} \mu_{\dot{\gamma}_{n-1}} .
\end{aligned}
$$

For the boundary integrals on the $t$-constant hypersurfaces, we will use (ii) of the following lemma.

Lemma 4.19 (boundary terms of $J^{(\alpha)}$ current on $t$-constant hypersurfaces). On each $\bar{\Sigma}_{t}$,

(i) there exists a constant $C(n, m, \alpha)$ such that

$$
\int_{\mathbb{R}}\left|\left(J^{(\alpha)}, T\right)\right| r^{n-1} \mathrm{~d} r^{*} \leq C(n, m, \alpha) \int_{\mathbb{R}}\left(J^{T}(\phi)+\sum_{i=1}^{\frac{n(n-1)}{2}} J^{T}\left(\Omega_{i} \phi\right), T\right) r^{n-1} \mathrm{~d} r^{*} ;
$$

(ii) for $r \geq r_{0}$ there exists a constant $C\left(n, m, \alpha, r_{0}\right)$ such that 


$$
\left|\left(J^{(\alpha)}, T\right)\right| \leq C\left(n, m, \alpha, r_{0}\right)\left(J^{T}(\phi)+\sum_{i=1}^{\frac{n(n-1)}{2}} J^{T}\left(\Omega_{i} \phi\right), T\right) .
$$

Proof. Using the definition (4-65),

$$
\begin{aligned}
& \left(J^{(\alpha)}, T\right) \\
& =f^{a}\left(\frac{\partial \phi}{\partial t}\right)\left(\frac{\partial \phi}{\partial r^{*}}\right)+\sum_{i=1}^{\frac{n(n-1)}{2}} f^{b}\left(\frac{\partial \Omega_{i} \phi}{\partial t}\right)\left(\frac{\partial \Omega_{i} \phi}{\partial r^{*}}\right)+\frac{1}{4} \sum_{i=1}^{\frac{n(n-1)}{2}}\left(\left(f^{b}\right)^{\prime}+(n-1) \frac{f^{b}}{r}\left(1-\frac{2 m}{r^{n-2}}\right)\right) 2\left(\Omega_{i} \phi\right)\left(\partial_{t} \Omega_{i} \phi\right)
\end{aligned}
$$

because

$$
\partial_{t}\left(\left(f^{b}\right)^{\prime}+(n-1) \frac{f^{b}}{r}\left(1-\frac{2 m}{r^{n-2}}\right)\right)=0
$$

and $g\left(T, \frac{\partial}{\partial r^{*}}\right)=0$. By Cauchy's inequality,

$$
\begin{aligned}
\left|\left(J^{(\alpha)}, T\right)\right| \leq \frac{C}{\alpha^{2} r^{n-1}}\left[\frac{1}{2}\left(\frac{\partial \phi}{\partial t}\right)^{2}+\right. & \left.\frac{1}{2}\left(\frac{\partial \phi}{\partial r^{*}}\right)^{2}\right]+\sum_{i=1}^{\frac{n(n-1)}{2}} \frac{\pi}{\alpha}\left[\frac{1}{2}\left(\frac{\partial \Omega_{i} \phi}{\partial t}\right)^{2}+\frac{1}{2}\left(\frac{\partial \Omega_{i} \phi}{\partial r^{*}}\right)^{2}\right] \\
& +\frac{1}{4} \sum_{i=1}^{\frac{n(n-1)}{2}}\left(\frac{r}{\alpha^{2}+x^{2}}+(n-1) \frac{\pi}{\alpha}\left(1-\frac{2 m}{r^{n-2}}\right)\right)\left[\frac{1}{r^{2}}\left(\Omega_{i} \phi\right)^{2}+\left(\frac{\partial \Omega_{i} \phi}{\partial t}\right)^{2}\right],
\end{aligned}
$$

which proves (ii) in view of

$$
\left(J^{T}(\phi), T\right)=\frac{1}{2}\left(\frac{\partial \phi}{\partial t}\right)^{2}+\frac{1}{2}\left(\frac{\partial \phi}{\partial r^{*}}\right)^{2}+\frac{1}{2}\left(1-\frac{2 m}{r^{n-2}}\right)|\not \nabla|^{2}
$$

here we have also used

$$
f^{b}=\int_{0}^{r^{*}} \frac{1}{\alpha^{2}+\left(t^{*}-\alpha-\sqrt{\alpha}\right)^{2}} \mathrm{~d} t^{*}=\left.\frac{1}{\alpha} \arctan x\right|_{\frac{-\alpha-\sqrt{\alpha}}{\alpha}} ^{\frac{r^{*}-\alpha-\sqrt{\alpha}}{\alpha}} \leq \frac{\pi}{\alpha} \quad\left(r^{*} \geq 0\right) .
$$

To establish (i) it is enough to infer

$$
\begin{aligned}
\int_{-\infty}^{\infty} \frac{r}{\alpha^{2}+x^{2}}|\nabla \phi|^{2} r^{n-1} \mathrm{~d} r^{*} & =\sum_{i=1}^{\frac{n(n-1)}{2}} \int_{-\infty}^{\infty} \frac{r^{n-2}}{\alpha^{2}+x^{2}}\left(\Omega_{i} \phi\right)^{2} \mathrm{~d} r^{*} \leq C \sum_{i=1}^{\frac{n(n-1)}{2}} \int_{-\infty}^{\infty}\left(\frac{\partial \Omega_{i} \phi}{\partial r^{*}}\right)^{2} r^{n-1} \mathrm{~d} r^{*} \\
& \leq C \sum_{i=1}^{\frac{n(n-1)}{2}} \int_{-\infty}^{\infty}\left(J^{T}\left(\Omega_{i} \phi\right), T\right) r^{n-1} \mathrm{~d} r^{*}
\end{aligned}
$$

this is a standard Hardy inequality; cf. proof of Proposition 10.2 in [Dafermos and Rodnianski 2009b].

The following lemma will be applied to the boundary terms of the $J^{(\alpha)}$-current on the null hypersurfaces in the region $r \leq r_{0}$. 
Lemma 4.20 (boundary terms of the $J^{(\alpha)}$ current on null hypersurfaces). (i) On any segment of the outgoing null hypersurface $u^{*}=u_{1}^{*} \geq 0$,

$$
\left|\left(J^{(\alpha)}, \frac{\partial}{\partial v^{*}}\right)\right| \leq C(n, m, \alpha)\left(J^{T}(\phi)+\sum_{i=1}^{\frac{n(n-1)}{2}} J^{T}\left(\Omega_{i} \phi\right), \frac{\partial}{\partial v^{*}}\right)+\epsilon\left(u_{1}^{*}\right)\left(J^{N}(\phi), \frac{\partial}{\partial v^{*}}\right),
$$

where $C(n, m, \alpha)$ is a constant, and $\epsilon\left(u_{1}^{*}\right) \rightarrow 0$ as $u_{1}^{*} \rightarrow \infty$.

(ii) Let $v_{0}^{*} \geq 1$, and $u_{0}^{*}\left(v^{*}\right)$ such that $r\left(u_{0}^{*}\left(v^{*}\right), v^{*}\right)=r_{0}$ (in particular $\left.u_{0}^{*}\left(v_{0}^{*}\right) \geq 1\right)$. Then, on the ingoing null hypersurface $v^{*}=v_{0}^{*}$,

$$
\int_{u_{0}^{*}}^{\infty}\left|\left(J^{(\alpha)}, \frac{\partial}{\partial u^{*}}\right)\right| r^{n-1} \mathrm{~d} u^{*} \leq C(n, m, \alpha) \int_{u_{0}^{*}}^{\infty}\left(J^{T}(\phi)+\sum_{i=1}^{\frac{n(n-1)}{2}} J^{T}\left(\Omega_{i} \phi\right), \frac{\partial}{\partial u^{*}}\right) r^{n-1} \mathrm{~d} u^{*} .
$$

Proof. Using the definition (4-65) we find

$$
\begin{aligned}
\left(J^{(\alpha)}, \frac{\partial}{\partial u^{*}}\right)=f^{a} T(\phi) & \left(\frac{\partial}{\partial r^{*}}, \frac{\partial}{\partial u^{*}}\right)+\sum_{i=1}^{\frac{n(n-1)}{2}}\left\{f^{b} T\left(\Omega_{i} \phi\right)\left(\frac{\partial}{\partial r^{*}}, \frac{\partial}{\partial u^{*}}\right)\right. \\
+ & \frac{1}{4}\left(\left(f^{b}\right)^{\prime}+(n-1) \frac{f^{b}}{r}\left(1-\frac{2 m}{r^{n-2}}\right)\right) 2\left(\Omega_{i} \phi\right)\left(\frac{\partial \Omega_{i} \phi}{\partial u^{*}}\right) \\
+ & \left.\frac{1}{4} \frac{1}{2}\left(\left(f^{b}\right)^{\prime}+(n-1) \frac{f^{b}}{r}\left(1-\frac{2 m}{r^{n-2}}\right)\right)^{\prime}\left(\Omega_{i} \phi\right)^{2}-\left(f^{b}\right)^{\prime} \beta\left(\Omega_{i} \phi\right)^{2}\right\},
\end{aligned}
$$

and therefore

$$
\begin{aligned}
& \left|\left(J^{(\alpha)}, \frac{\partial}{\partial u^{*}}\right)\right| \\
& \leq \frac{C}{\alpha^{2}(2 m)^{\frac{n-1}{n-2}}}\left[\frac{1}{2}\left(\frac{\partial \phi}{\partial u^{*}}\right)^{2}+\frac{1}{2}\left(1-\frac{2 m}{r^{n-2}}\right)|\not \nabla \phi|^{2}\right]+\sum_{i=1}^{\frac{n(n-1)}{2}} \frac{\pi}{\alpha}\left[\frac{1}{2}\left(\frac{\partial \Omega_{i} \phi}{\partial u^{*}}\right)^{2}+\frac{1}{2}\left(1-\frac{2 m}{r^{n-2}}\right)\left|\not \Omega_{i} \phi\right|^{2}\right] \\
& \quad+\sum_{i=1}^{\frac{n(n-1)}{2}} \frac{1}{2}\left(\frac{r}{\alpha^{2}+x^{2}}+(n-1) \frac{\pi}{\alpha}\left(1-\frac{2 m}{r^{n-2}}\right)\right) \frac{1}{2}\left(\frac{\partial \Omega_{i} \phi}{\partial u^{*}}\right)^{2} \\
& \quad+\left(\frac{n-1}{2} \frac{\pi}{\alpha}+\frac{n-1}{4} \frac{r}{\alpha^{2}+x^{2}}+\frac{n-1}{4} \frac{\pi}{\alpha}\left(1-\frac{2 m}{r^{n-2}}\right)+\frac{(n-1)(n-2)}{4} \frac{\pi}{\alpha}+(n-1) \frac{r}{\alpha^{2}+x^{2}}\right) \frac{1}{2}\left(1-\frac{2 m}{r^{n-2}}\right)|\nabla \phi|^{2} \\
& \quad+\left(\frac{1}{4} \frac{r}{\alpha^{2}+x^{2}}+\frac{5}{4} \frac{|x| r^{2}}{\left(\alpha^{2}+x^{2}\right)^{2}}\right)|\nabla \phi|^{2} .
\end{aligned}
$$

Similarly for $\left|\left(J^{(\alpha)}, \frac{\partial}{\partial v^{*}}\right)\right|$. Clearly, (ii) now follows from

$$
\left(J^{T}, \frac{\partial}{\partial v^{*}}\right)=\frac{1}{2}\left(\frac{\partial \phi}{\partial v^{*}}\right)^{2}+\frac{1}{2}\left(1-\frac{2 m}{r^{n-2}}\right)|\not \nabla \phi|^{2}
$$


and

$$
\left(J^{N}, \frac{\partial}{\partial v^{*}}\right)=\left[1+\frac{\sigma}{4 \kappa}\left(1-\frac{2 m}{r^{n-2}}\right)\right] T\left(\frac{2}{1-\frac{2 m}{r^{n-2}}} \frac{\partial}{\partial u^{*}}+\frac{\partial}{\partial t}, \frac{\partial}{\partial v^{*}}\right) \geq 2|\not \phi|^{2}+\frac{1}{2}\left(\frac{\partial \phi}{\partial v^{*}}\right)^{2} .
$$

In case (i) we only have

$$
\left(J^{T}, \frac{\partial}{\partial u^{*}}\right)=\frac{1}{2}\left(\frac{\partial \phi}{\partial u^{*}}\right)^{2}+\frac{1}{2}\left(1-\frac{2 m}{r^{n-2}}\right)|\not \nabla|^{2}, \quad\left(J^{N}, \frac{\partial}{\partial u^{*}}\right) \geq \frac{1}{2}\left(1-\frac{2 m}{r^{n-2}}\right)|\nabla \phi|^{2} ;
$$

but, using the Hardy inequality of Lemma C.7,

$$
\begin{aligned}
\int_{u_{0}^{*}}^{\infty} \frac{r}{\alpha^{2}+x^{2}}|\mathbb{\phi} \phi|^{2} r^{n-1} \mathrm{~d} u^{*} \leq & \sum_{i=1}^{\frac{n(n-1)}{2}} \int_{u_{0}^{*}}^{\infty} \frac{1+u^{* 2}}{\alpha^{2}+\left(u^{*}+\alpha+\sqrt{\alpha}-v^{*}\right)^{2}} r_{0}^{n-2} \frac{1}{1+u^{* 2}}\left(\Omega_{i} \phi\right)^{2} \mathrm{~d} u^{*} \\
\leq & 8 C(n, m, \alpha) \frac{1+u_{0}^{* 2}}{u_{0}^{* 2}} \sum_{i=1}^{\frac{n(n-1)}{2}} \int_{u_{0}^{*}}^{\infty}\left(\frac{\partial \Omega_{i} \phi}{\partial u^{*}}\right)^{2} r^{n-1} \mathrm{~d} u^{*} \\
& +2 \pi C(n, m, \alpha) \sum_{i=1}^{\frac{n(n-1)}{2}} \int_{u_{0}^{*}}^{u_{0}^{*}+1}\left\{\left(\Omega_{i} \phi\right)^{2}+\left(\frac{\partial \Omega_{i} \phi}{\partial u^{*}}\right)^{2}\right\} \mathrm{d} u^{*} \\
& \leq C(n, m, \alpha) \int_{u_{0}^{*}}^{\infty}\left(J^{T}(\phi)+\sum_{i=1}^{n(n-1)} J^{T}\left(\Omega_{i} \phi\right), \frac{\partial}{\partial u^{*}}\right) r^{n-1} \mathrm{~d} u^{*} .
\end{aligned}
$$

Obviously the same bound holds for

$$
\int_{u_{0}^{*}}^{\infty} \frac{|x| r^{2}}{\left(\alpha^{2}+x^{2}\right)^{2}}|\not \nabla|^{2} r^{n-1} \mathrm{~d} u^{*}
$$

Step $2 b$ (commutation with $\Omega_{i}$ ). Since

$$
\begin{gathered}
{\left[\Omega_{i}, \frac{\partial}{\partial t}\right]=0,} \\
\sum_{i=1}^{\frac{n(n-1)}{2}}\left(\frac{\partial}{\partial t} \Omega_{i} \cdot \pi_{<L} \phi\right)^{2}=r^{2}\left|\not \frac{\partial \pi_{<L} \phi}{\partial t}\right|_{r^{2} \dot{\gamma}_{n-1}}^{2},
\end{gathered}
$$

and since also

$$
\left[\Omega_{i}, \frac{\partial}{\partial r}\right]=0
$$

we have

$$
\sum_{i=1}^{\frac{n(n-1)}{2}}\left(\frac{\partial}{\partial r^{*}} \Omega_{i} \cdot \pi_{<L} \phi\right)^{2}=r^{2}\left|\not \frac{\partial \pi_{<L} \phi}{\partial r^{*}}\right|^{2}
$$


Moreover

so that

$$
\left[\pi_{l}, \frac{\partial}{\partial t}\right]=0
$$

$$
\int_{S_{r}} \sum_{i=1}^{\frac{n(n-1)}{2}}\left(\frac{\partial}{\partial t} \Omega_{i} \cdot \pi_{<L} \phi\right)^{2} \mathrm{~d} \mu_{\gamma}=\int_{S_{r}} r^{2}\left|\not \frac{\partial \pi_{<L} \phi}{\partial t}\right|^{2} \mathrm{~d} \mu_{\gamma} \leq L(L+n+2) \int_{S_{r}}\left(\frac{\partial \pi_{<L} \phi}{\partial t}\right)^{2} \mathrm{~d} \mu_{\gamma} .
$$

Since also

$$
\int_{S_{r}}\left|\not \nabla \Omega_{i} \cdot \pi_{<L} \phi\right|_{r^{2} \dot{\gamma}_{n-1}}^{2} \mathrm{~d} \mu_{\gamma} \leq \frac{L(L+n-2)}{r^{2}} \int_{S_{r}}\left(\Omega_{i} \cdot \pi_{<L} \phi\right)^{2} \mathrm{~d} \mu_{\gamma}
$$

we have

$$
\int_{S_{r}} \sum_{i=1}^{\frac{n(n-1)}{2}}\left|\nabla \Omega_{i} \cdot \pi_{<L} \phi\right|_{r^{2} \dot{\gamma}_{n-1}}^{2} \mathrm{~d} \mu_{\gamma} \leq L(L+n-2) \int_{S_{r}}\left|\nabla \pi_{<L} \phi\right|_{r^{2} \dot{\gamma}_{n-1}}^{2} \mathrm{~d} \mu_{\gamma}
$$

Therefore indeed,

$$
\begin{aligned}
& \int_{S_{r}} \sum_{i=1}^{\frac{n(n-1)}{2}}\left(J^{T}\left(\Omega_{i} \cdot \pi_{<L} \phi\right), \frac{\partial}{\partial t}\right) \\
& \quad=\frac{1}{2} \sum_{i=1}^{\frac{n(n-1)}{2}} \int_{S_{r}}\left\{\left(\frac{\partial \Omega_{i} \cdot \pi_{<L} \phi}{\partial t}\right)^{2}+\left(\frac{\partial \Omega_{i} \cdot \pi_{<L} \phi}{\partial r^{*}}\right)^{2}+\left(1-\frac{2 m}{r^{n-2}}\right)\left|\nabla \Omega_{i} \cdot \pi_{<L} \phi\right|_{r^{2} \dot{\gamma}_{n-1}}^{2}\right\} \mathrm{d} \mu_{\gamma} \\
& \quad \leq \frac{1}{2}(6 \gamma n)^{2}\left((6 \gamma n)^{2}+n-2\right) \int_{S_{r}}\left(J^{T}\left(\pi_{<L} \phi\right), \frac{\partial}{\partial t}\right) \mathrm{d} \mu_{\gamma}
\end{aligned}
$$

because $L=(6 \gamma n)^{2}$ is fixed; similarly, of course, for $\left(J^{T}, \frac{\partial}{\partial u^{*}}\right)$ and $\left(J^{T}, \frac{\partial}{\partial v^{*}}\right)$.

We conclude the statement of the proposition with the treatment of the two regimes in Steps 1 and Step 2 above from

$$
\begin{aligned}
\int_{\mathcal{R}_{r_{0}, r_{1}}^{\infty}\left(t_{0}\right)} & \left\{\frac{1}{r^{n}}\left(\frac{\partial \phi}{\partial r^{*}}\right)^{2}+\frac{1}{r^{n+1}}\left(\frac{\partial \phi}{\partial t}\right)^{2}+\frac{1}{r^{3}}\left(1-\frac{2 m}{r^{n-2}}\right)|\not \phi|_{r^{2} \dot{\gamma}_{n-1}}^{2}\right\} \\
\leq & 2 \int_{\mathcal{R}_{r_{0}, r_{1}}^{\infty}\left(t_{0}\right)}\left\{\frac{1}{r^{n}}\left(\frac{\partial \pi_{<L} \phi}{\partial r^{*}}\right)^{2}+\frac{1}{r^{n+1}}\left(\frac{\partial \pi_{<L} \phi}{\partial t}\right)^{2}+\frac{1}{r^{2}}\left|\nabla \pi_{<L} \phi\right|_{r^{2} \dot{\gamma}_{n-1}}^{2}\right\} \\
& +2 \int_{\mathcal{R}_{r_{0}, r_{1}}^{\infty}\left(t_{0}\right)}\left\{\frac{1}{r^{3}}\left(\frac{\partial \pi_{\geq L} \phi}{\partial r^{*}}\right)^{2}+\frac{1}{r^{3}}\left(\frac{\partial \pi_{\geq L} \phi}{\partial t}\right)^{2}+\frac{1}{r^{3}}\left(1-\frac{2 m}{r^{n-2}}\right)\left|\nabla \pi_{\geq L} \phi\right|_{r^{2} \dot{\gamma}_{n-1}}^{2}\right\} .
\end{aligned}
$$

4D.2. Refinement for finite regions. In the proof of Proposition 4.1, neither of the currents used for the high or the low spherical harmonic regime requires the use of Hardy inequalities for the boundary integrals in the asymptotic region; indeed in both cases the zeroth-order terms $\phi^{2}$ can be estimated by the angular derivatives $|\not \nabla \phi|^{2}$, in the case of the current $J^{X_{\gamma, \alpha}, 1}$ for high angular frequencies by Poincaré's inequality Lemma 4.6, and in the case of the current $J^{(\alpha)}$ for low angular frequencies as a result of the commutation with $\Omega_{i}$ in (4-65). Therefore we can in fact state a refinement of Proposition 4.1 for finite regions, i.e., an 


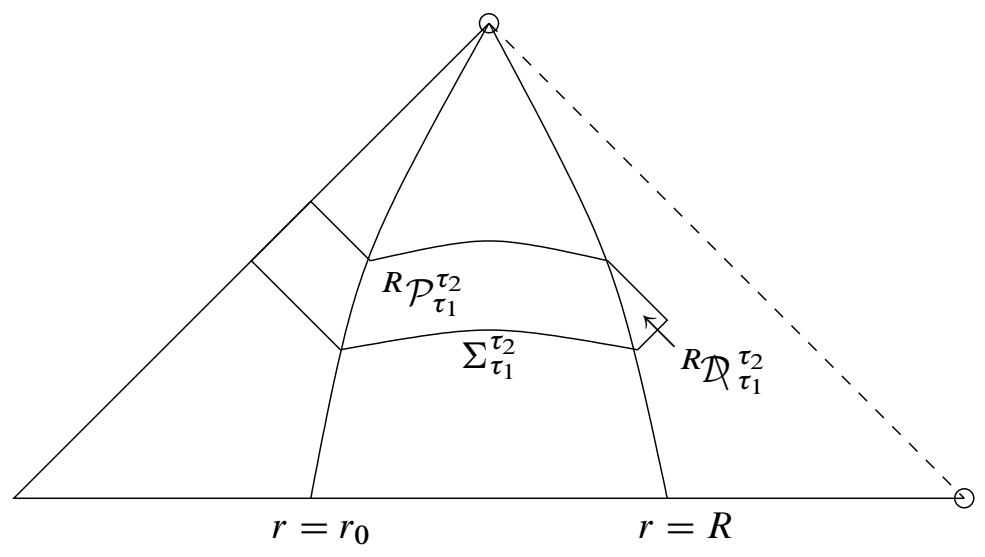

Figure 6. The past boundary $\Sigma_{\tau_{1}}^{\tau_{2}}$ of $R_{\mathcal{P}_{\tau_{1}}}^{\tau_{2}} \cup{ }^{R} \mathcal{Q}_{\tau_{1}}^{\tau_{2}}$.

integrated local energy estimate on bounded domains in terms of the flux through the past boundary of that domain, that will be relevant in Section $5 \mathrm{C}$.

Let

$$
\begin{aligned}
{ }^{R} \mathcal{P}_{\tau_{1}}^{\tau_{2}} & \doteq \mathcal{R}_{r_{0}, R}^{\infty}\left(2 \tau_{1}+R^{*}, 2 \tau_{2}+R^{*}\right) \cap\{r \leq R\}, \\
{ }^{R} \mathcal{Q}_{\tau_{1}}^{\tau_{2}} & \doteq\left\{\left(u^{*}, v^{*}\right): \tau_{1} \leq u^{*} \leq \tau_{2}, v^{*}-u^{*} \geq R^{*}, v^{*} \leq \tau_{2}+R^{*}\right\} \\
& =\mathcal{R}_{r_{0}, R}^{\infty}\left(2 \tau_{1}+R^{*}, 2 \tau_{2}+R^{*}, \tau_{2}+\frac{1}{2}\left(R^{*}-r_{0}^{*}\right), \tau_{2}+R^{*}\right) \backslash R \mathcal{P}_{\tau_{1}}^{\tau_{2}},
\end{aligned}
$$

and denote by $\Sigma_{\tau_{1}}^{\tau_{2}}$ the past boundary of ${ }^{R} \mathcal{P}_{\tau_{1}}^{\tau_{2}} \cup{ }^{R} \mathcal{D}_{\tau_{1}}^{\tau_{2}}$ (see also Figure 6):

$$
\begin{aligned}
& \Sigma_{\tau_{1}}^{\tau_{2}} \doteq \partial^{-}\left({ }^{R} \mathcal{P}_{\tau_{1}}^{\tau_{2}} \cup{ }^{R} \mathcal{Q}_{\tau_{1}}^{\tau_{2}}\right)=\{\left\{\left(u^{*}, v^{*}\right): v^{*}=\tau_{1}+\frac{1}{2}\left(R^{*}+r_{0}^{*}\right), u^{*} \geq \tau_{1}+\frac{1}{2}\left(R^{*}-r_{0}^{*}\right)\right\} \\
& \cup\left\{\left(u^{*}, v^{*}\right): u^{*}+v^{*}=2 \tau_{1}+R^{*}, r_{0}^{*} \leq v^{*}-u^{*} \leq R^{*}\right\} \\
& \cup\left\{\left(u^{*}, v^{*}\right): u^{*}=\tau_{1}, R^{*}+\tau_{1} \leq v^{*} \leq R^{*}+\tau_{2}\right\} .
\end{aligned}
$$

Proposition 4.21 (integrated local energy decay on finite regions). Let $\phi$ be a solution of the wave equation $\square_{g} \phi=0$, and $R>\sqrt[n-2]{2 m}$. Then there exists a constant $C(n, m, R)$, such that, for any $\tau_{2}>\tau_{1}$,

$$
\begin{aligned}
\int_{R \mathcal{P}_{\tau_{1}}^{\tau_{2}}}\left\{\left(\frac{\partial \phi}{\partial r^{*}}\right)^{2}+\left(\frac{\partial \phi}{\partial t}\right)^{2}+\left(1-\frac{2 m}{r^{n-2}}\right)|\not \nabla \phi|_{r^{2} \dot{\gamma}_{n-1}}^{2}\right\} \mathrm{d} \mu_{g} & \\
& \leq C(n, m, R) \int_{\Sigma_{\tau_{1}}^{\tau_{2}}}\left(J^{T}(\phi)+J^{T}(T \cdot \phi), n\right) .
\end{aligned}
$$

In view of the remarks above, the proof of Proposition 4.21 is of course identical to the proof of Proposition 4.1 given in Section 4D.1 by replacing the unbounded domain $\mathcal{R}_{r_{0}, r_{1}}^{\infty}\left(2 \tau_{1}+R^{*}\right)$ by the bounded domain ${ }^{R} \mathcal{P}_{\tau_{1}}^{\tau_{2}} \cup{ }^{R} \mathcal{Q}_{\tau_{1}}^{\tau_{2}}$.

However, this estimate does not include the zeroth-order term, which we have covered separately in Proposition 4.4. 
Proposition 4.22 (refinement for zeroth-order terms on timelike boundaries). Let $\phi$ be solution of the wave equation (1-1), and $R>\sqrt[n-2]{8 n m}$. Then there is a constant $C(n, m, R)$ such that for all $\tau^{\prime}<\tau$,

$$
\begin{aligned}
\left.\int_{2 \tau^{\prime}+R^{*}}^{2 \tau+R^{*}} \mathrm{~d} t \int_{\mathbb{S}^{n-1}} \mathrm{~d} \mu_{\dot{\gamma}_{n-1}} \phi^{2}\right|_{r=R} \leq C(n, m, R) & \left\{\left.\int_{2 \tau^{\prime}+R^{*}}^{2 \tau+R^{*}} \mathrm{~d} t \int_{\mathbb{S}^{n-1}} \mathrm{~d} \mu_{\dot{\gamma}_{n-1}}\left\{\left(\frac{\partial \phi}{\partial r^{*}}\right)^{2}+|\not \phi|^{2}\right\}\right|_{r=R}\right. \\
& \left.+\int_{\Sigma_{\tau^{\prime}}^{\tau}}\left(J^{T}(\phi), n\right)+\left.\int_{\mathbb{S}^{n-1}} \mathrm{~d} \mu_{\dot{\gamma}_{n-1}} r^{n-2} \phi^{2}\right|_{\left(\tau^{\prime}, R^{*}+\tau\right)}\right\} .
\end{aligned}
$$

The proof remains the same as for Proposition 4.4 on page 534 with the exception that we consider the energy identity for $J^{X, 1}$ on ${ }^{R} \mathcal{Q} \tau_{\tau^{\prime}}^{\tau}$ in place of ${ }^{R} \mathcal{D}_{\tau^{\prime}}^{\tau}$ and use Proposition C.5 instead of Proposition C.1.

\section{The decay argument}

We will here prove energy decay of the solutions to the wave equation and higher-order energy decay of their time derivatives in the interior based on the integrated local energy decay statements of Section 4, following the new physical-space approach to decay of [Dafermos and Rodnianski 2010].

Remark 5.1. Instead one could use the conformal Morawetz vector field

$$
Z=u^{* 2} \frac{\partial}{\partial u^{*}}+v^{* 2} \frac{\partial}{\partial v^{*}}
$$

to prove energy decay of solutions to the wave equation with a rate corresponding to the weights in $Z$; this is done in [Schlue 2010]. Similarly the use of the scaling vector field

$$
S=v^{*} \frac{\partial}{\partial v^{*}}+u^{*} \frac{\partial}{\partial u^{*}}
$$

should provide an alternative approach to prove higher-order energy decay [Luk 2010]. Here however, we shall avoid the use of multipliers with weights in $t$.

5A. Uniform boundedness. A preliminary feature of the solutions to the wave equation (1-1) that is necessary to employ the decay mechanism of [Dafermos and Rodnianski 2010] is the uniform boundedness of their (nondegenerate) energy; this is a consequence of the conservation of the degenerate energy associated to the multiplier $T$, and the redshift effect of Section 3, which allows us to control the nondegenerate energy on the horizon.

Let $\Sigma$ be a (spherically symmetric) spacelike hypersurface in $\mathcal{M}, \Sigma^{\prime}=\Sigma \cap\{r \leq R\}$ and $\mathcal{N}$ the outgoing null hypersurface emerging from $\partial \Sigma^{\prime}$ (Figure 7). Moreover, let

$$
\Sigma_{\tau}=\varphi_{\tau}\left(\left(\Sigma^{\prime} \cup \mathcal{N}\right) \cap \mathcal{D}\right), \quad \Sigma_{\tau}^{\prime}=\Sigma_{\tau} \cap\{r \leq R\}, \quad \Sigma_{\tau^{\prime}}^{\tau}=\Sigma_{\tau^{\prime}} \cap J^{-}\left(\Sigma_{\tau}^{\prime}\right) .
$$

Proposition 5.2 (uniform boundedness). Let $\phi$ be a solution of the wave equation (1-1) with initial data on $\Sigma_{0}$. Then there exists a constant $C\left(\Sigma_{0}\right)$ such that

$$
\int_{\Sigma_{\tau}^{\prime}}\left(J^{N}(\phi), n\right) \leq C \int_{\Sigma_{0}^{\tau}}\left(J^{N}(\phi), n\right) \quad(\tau>0) .
$$




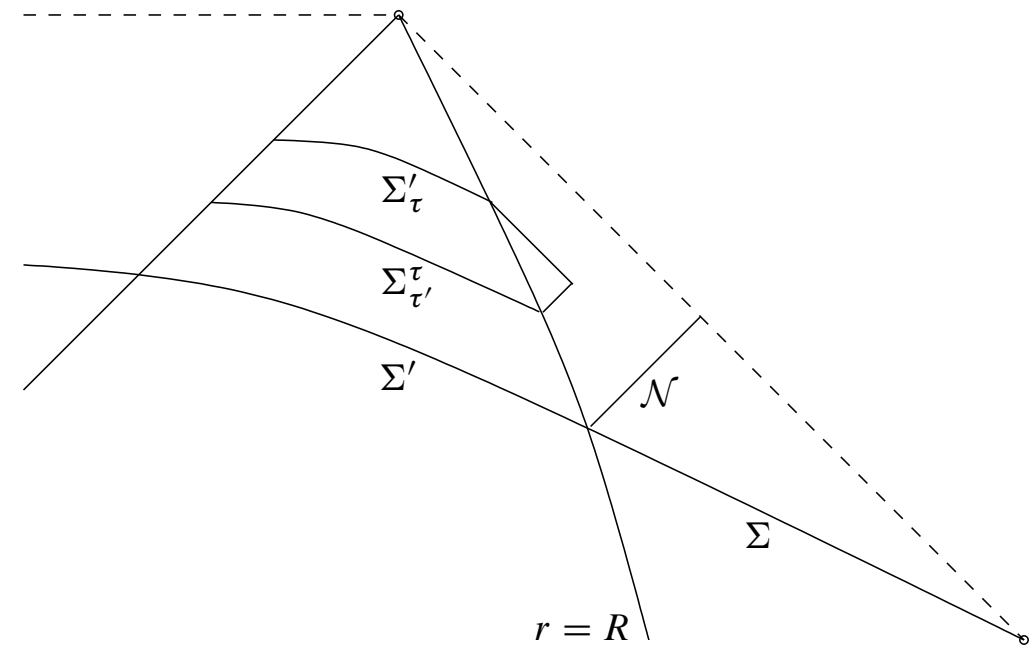

Figure 7. The construction of the surfaces $\Sigma_{\tau}^{\prime}$ from $\Sigma$.

Proof. One can proceed in analogy to the local observer's energy estimate of [Dafermos and Rodnianski 2008]; indeed, from the energy identity for $N$ on the domain $\mathcal{R}\left(\tau^{\prime}, \tau\right)=\cup_{\tau^{\prime} \leq \bar{\tau} \leq \tau} \Sigma_{\bar{\tau}}^{\tau}$ it follows

$$
\int_{\Sigma_{\tau}^{\prime}}\left(J^{N}, n\right)+\int_{\mathcal{R}\left(\tau^{\prime}, \tau\right)} K^{N} \leq \int_{\Sigma_{\tau^{\prime}}^{\tau}}\left(J^{N}, n\right)
$$

since $\left(J^{N}, n_{\mathcal{H}}\right) \geq 0$, and $\left(J^{N}, n_{\mathcal{N}}\right) \geq 0$. By Proposition 3.3, namely the redshift effect, $K^{N}$ is bounded from below by $\left(J^{N}, n\right)$ near the horizon, and from above by $\left(J^{T}, n\right)$ away from the horizon; since also the lapse of the foliation of $\mathcal{R}$ is bounded from above and below we conclude that there are constants $0<b<B$ only depending on $\Sigma$ and $N$ such that

$$
\begin{aligned}
\int_{\Sigma_{\tau}^{\prime}}\left(J^{N}, n\right)+b \int_{\tau^{\prime}}^{\tau} \mathrm{d} \bar{\tau} \int_{\Sigma_{\bar{\tau}}^{\tau}}\left(J^{N}, n\right) \leq B \int_{\tau^{\prime}}^{\tau} \mathrm{d} \bar{\tau} \int_{\Sigma_{\bar{\tau}}^{\tau}}\left(J^{T}, n\right)+\int_{\Sigma_{\tau^{\prime}}^{\tau}}\left(J^{N}, n\right) \\
\leq B\left(\tau-\tau^{\prime}\right) \int_{\Sigma_{\tau^{\prime}}^{\tau}}\left(J^{T}, n\right)+\int_{\Sigma_{\tau^{\prime}}^{\tau}}\left(J^{N}, n\right),
\end{aligned}
$$

where in the last step we have used the energy identity for $T$ on $\mathcal{R}\left(\tau^{\prime}, \bar{\tau}\right)$ and $K^{T}=0$. Thus the desired energy bound follows from the elementary Lemma 5.3.

Lemma 5.3. Let $f: \mathbb{R} \rightarrow \mathbb{R}$ be a nonnegative function, $f \geq 0$, such that for all $t_{1} \leq t_{2}$ and two positive constants $0<c<C$,

$$
f\left(t_{2}\right)+c \int_{t_{1}}^{t_{2}} f(t) \mathrm{d} t \leq C\left(t_{2}-t_{1}\right)+f\left(t_{1}\right) .
$$

Then

$$
f\left(t_{2}\right) \leq f\left(t_{1}\right)+\frac{C}{c} \quad\left(t_{2} \geq t_{1}\right) .
$$

Proof. See, e.g., [Schlue 2012]. 
5B. Energy decay. In this section we prove quadratic decay of the nondegenerate energy.

Let

$$
\Sigma_{\tau_{0}} \doteq \partial^{-} \mathcal{R}_{r_{0}, R}^{\infty}\left(t_{0}\right), \quad \tau_{0}=\frac{1}{2}\left(t_{0}-R^{*}\right),
$$

with $R>\sqrt[n-2]{8 n m}, t_{0}>0$ and $r_{0} \doteq r_{0}^{(N)}$ according to Proposition 3.1.

Proposition 5.4 (energy decay). Let $\phi$ be a solution of the wave equation (1-1) with initial data on $\Sigma_{\tau_{0}}$ satisfying

$$
\left.D \doteq \int_{\tau_{0}+R^{*}}^{\infty} \mathrm{d} v \int_{\mathbb{S}^{n-1}} \mathrm{~d} \mu_{\gamma_{n-1}} \sum_{k=0}^{1} r^{2}\left(\frac{\partial r^{\frac{n-1}{2}} \partial_{t}^{k} \phi}{\partial v^{*}}\right)^{2}\right|_{u=\tau_{0}}+\int_{\Sigma_{\tau_{0}}}\left(\sum_{k=0}^{2} J^{N}\left(T^{k} \cdot \phi\right), n\right)<\infty .
$$

Then there exists a constant $C(n, m, R)$ such that

$$
\int_{\Sigma_{\tau}}\left(J^{N}(\phi), n\right) \leq \frac{C D}{\tau^{2}} \quad\left(\tau>\tau_{0}\right)
$$

The proof is based on a weighted energy inequality, derived from the energy identity for the current (5-8) on the domain

$$
{ }^{R} \mathcal{D}_{\tau_{1}}^{\tau_{2}}=\left\{\left(u^{*}, v^{*}\right): \tau_{1} \leq u^{*} \leq \tau_{2}, v^{*}-u^{*} \geq R^{*}\right\} .
$$

Weighted energy identity. Consider the current

$$
\stackrel{r}{J}_{\mu}(\phi)=T_{\mu \nu}(\psi) V^{\nu}
$$

where

$$
\begin{gathered}
\psi=r^{\frac{n-1}{2} \phi}, \\
V=r^{q} \frac{\partial}{\partial v^{*}}, \quad q=p+1-n, \quad p \in\{1,2\} .
\end{gathered}
$$

This may also be viewed as the current to the multiplier vector field $r^{p} \frac{\partial}{\partial v^{*}}$, modified by the following terms:

$$
\begin{aligned}
\stackrel{J}{J}_{\mu}(\phi)=T_{\mu \nu}(\phi) & r^{p}\left(\frac{\partial}{\partial v^{*}}\right)^{v}+\left(\frac{n-1}{2}\right)^{2} r^{p-2}\left(1-\frac{2 m}{r^{n-2}}\right)\left(\partial_{\mu} r\right) \phi^{2} \\
+ & \frac{1}{2} \frac{n-1}{2} r^{p-1}\left(\partial_{\mu} r\right) \frac{\partial \phi^{2}}{\partial v^{*}}+\frac{1}{2} \frac{n-1}{2} r^{p-1}\left(1-\frac{2 m}{r^{n-2}}\right)\left(\partial_{\mu} \phi^{2}\right) \\
& \quad-\frac{1}{2}\left(\frac{n-1}{2}\right)^{2} r^{p-2}\left(1-\frac{2 m}{r^{n-2}}\right)\left(\frac{\partial}{\partial v^{*}}\right)_{\mu} \phi^{2}-\frac{1}{2} \frac{n-1}{2}\left(\frac{\partial}{\partial v^{*}}\right)_{\mu} r^{p-1} \frac{\partial \phi^{2}}{\partial r^{*}} .
\end{aligned}
$$

If $\square_{g} \phi=0$ then we calculate

$$
\begin{aligned}
\square_{g} \psi & =-\left(1-\frac{2 m}{r^{n-2}}\right)^{-1} \partial_{u^{*}} \partial_{v^{*}} \psi+\frac{n-1}{r} \frac{\partial \psi}{\partial r^{*}}+\frac{1}{r^{2}} \stackrel{\bigotimes}{4}_{n-1} \psi \\
& =\frac{n-1}{2}\left(\frac{n-3}{2}+\frac{n-1}{2} \frac{2 m}{r^{n-2}}\right) \frac{1}{r^{2}} \psi+\frac{n-1}{r} \frac{\partial}{\partial r^{*}} \psi .
\end{aligned}
$$


So the wave equation for $\phi$,

$$
\square_{g} \phi=0,
$$

is equivalent to the following equation for $\psi$ :

$$
-\partial_{u^{*}} \partial_{v^{*}} \psi+\left(1-\frac{2 m}{r^{n-2}}\right) \frac{1}{r^{2}} \stackrel{\leftrightarrow}{4}_{n-1} \psi-\frac{n-1}{2}\left(\frac{n-3}{2}+\frac{n-1}{2} \frac{2 m}{r^{n-2}}\right) \frac{1}{r^{2}}\left(1-\frac{2 m}{r^{n-2}}\right) \psi=0 .
$$

Now,

$$
\stackrel{r}{K}(\phi)=\nabla^{\mu} \stackrel{r}{J}_{\mu}(\phi)=\square_{g}(\psi) V \cdot \psi+K^{V}(\psi),
$$

where

$$
K^{V}(\psi)={ }^{(V)} \pi^{\mu \nu} T_{\mu \nu}(\psi)
$$

Since

$$
\begin{aligned}
{ }^{(V)} \pi_{u^{*} u^{*}} & =2 q r^{q-1}\left(1-\frac{2 m}{r^{n-2}}\right)^{2}, \\
{ }^{(V)} \pi_{v^{*} v^{*}} & =0, \\
{ }^{(V)} \pi_{u^{*} v^{*}} & =-\left(1-\frac{2 m}{r^{n-2}}\right) r^{q-1}\left[q+(n-q-2) \frac{2 m}{r^{n-2}}\right], \\
{ }^{(V)} \pi_{a A} & =0, \\
(V) & \pi_{A B}=r^{q-1}\left(1-\frac{2 m}{r^{n-2}}\right) g_{A B},
\end{aligned}
$$

we find

$$
\begin{aligned}
K^{r} \cdot r^{n-1}=\frac{n-1}{4}\left(\frac{n-3}{2}+\frac{n-1}{2} \frac{2 m}{r^{n-2}}\right) \frac{r^{p}}{r^{2}} \frac{\partial \psi^{2}}{\partial v^{*}} & +\frac{p}{2} r^{p-1}\left(\frac{\partial \psi}{\partial v^{*}}\right)^{2} \\
& +\frac{1}{2} r^{p-1}\left[(2-p)+(p-n) \frac{2 m}{r^{n-2}}\right]|\nabla \psi|_{r^{2} \dot{\gamma}_{n-1}}^{2}
\end{aligned} .
$$

One may integrate the first term by parts to obtain

$$
\begin{aligned}
\int_{u^{*}+R^{*}}^{\infty} \mathrm{d} v^{*} \stackrel{r}{K} \cdot r^{n-1}=\frac{n-1}{4} & \left.\left(\frac{n-3}{2}+\frac{n-1}{2} \frac{2 m}{r^{n-2}}\right) \frac{r^{p}}{r^{2}} \psi^{2}\right|_{u^{*}+R^{*}} ^{\infty} \\
+ & \int_{u^{*}+R^{*}}^{\infty} \mathrm{d} v^{*}\left\{\left[\frac{n-1}{4}(2-p) \frac{n-3}{2}+\frac{n-1}{2}(n-p) \frac{2 m}{r^{n-2}}\right] \frac{r^{p}}{r^{3}}\left(1-\frac{2 m}{r^{n-2}}\right) \psi^{2}\right. \\
+ & \left.\frac{p}{2} r^{p-1}\left(\frac{\partial \psi}{\partial v^{*}}\right)^{2}+\frac{1}{2} r^{p-1}\left[2-p+(p-n) \frac{2 m}{r^{n-2}}\right]|\nabla \psi|_{r^{2} \dot{\gamma}_{n-1}}^{2}\right\} \cdot
\end{aligned}
$$

We can now write down the energy identity for the current $\stackrel{r}{J}$ (see also Appendix B): 


$$
\int_{R_{\mathcal{D}_{\tau_{1}}}^{\tau_{2}}} \stackrel{r}{K} \mathrm{~d} \mu_{g}=\int_{\partial^{R} \mathcal{D}_{\tau_{1}}^{\tau_{2}}} *^{r}
$$

Dropping the positive zeroth-order terms, we obtain

$$
\begin{aligned}
& \left.\int_{\tau_{2}+R^{*}}^{\infty} \mathrm{d} v^{*} \int_{\mathbb{S}^{n-1}} \mathrm{~d} \mu_{\dot{\gamma}_{n-1}} \frac{1}{2} r^{p}\left(\frac{\partial \psi}{\partial v^{*}}\right)^{2}\right|_{u^{*}=\tau_{2}} \\
& \quad+\int_{\tau_{1}}^{\tau_{2}} \mathrm{~d} u^{*} \int_{u^{*}+R^{*}}^{\infty} \mathrm{d} v^{*} \int_{\mathbb{S}^{n-1}} \mathrm{~d} \mu_{\dot{\gamma}_{n-1}}\left\{\frac{p}{2} r^{p-1}\left(\frac{\partial \psi}{\partial v^{*}}\right)^{2}+\frac{1}{2} r^{p-1}\left[2-p+(p-n) \frac{2 m}{r^{n-2}}\right]|\not \psi|_{r^{2} \dot{\gamma}_{n-1}}^{2}\right\} \\
& \quad+\left.\int_{\tau_{1}}^{\tau_{2}} \mathrm{~d} u^{*} \int_{\mathbb{S}^{n-1}} \mathrm{~d} \mu_{\dot{\gamma}_{n-1}} \frac{1}{2} r^{p}|\not \nabla \psi|_{r^{2} \dot{\gamma}_{n-1}}^{2}\right|_{v^{*} \rightarrow \infty} \\
& \leq\left(1-\frac{2 m}{R^{n-2}}\right)^{-1}\left\{\left.\int_{\tau_{1}+R^{*}}^{\infty} \mathrm{d} v^{*} \int_{\mathbb{S}^{n-1}} \mathrm{~d} \mu_{\dot{\gamma}_{n-1}} \frac{1}{2} r^{p}\left(\frac{\partial \psi}{\partial v^{*}}\right)^{2}\right|_{u^{*}=\tau_{1}}\right. \\
& \quad+\int_{2 \tau_{1}+R^{*}}^{2 \tau_{2}+R^{*}} \mathrm{~d} t \int_{\mathbb{S}^{n-1}} \mathrm{~d} \mu_{\dot{\gamma}_{n-1}}\left[\frac{1}{4} r^{p}\left(\frac{\partial \psi}{\partial v^{*}}\right)^{2}+\frac{1}{4} r^{p}|\not \nabla \psi|_{r^{2} \dot{\gamma}_{n-1}}^{2}\right. \\
& \left.\left.+\frac{n-1}{4} \frac{1}{2} r^{p}\left(\frac{n-3}{2}+\frac{n-1}{2} \frac{2 m}{R^{n-2}}\right) \frac{1}{r^{2}} \psi^{2}\right]\left.\right|_{r=R}\right\} . \text { (5-17) }
\end{aligned}
$$

Note that the powers of $r$ that appear in the bulk term are 1 less than those that appear in the boundary terms. This allows for a hierarchy of inequalities (5-17) for different values of $p$, the so-called p-hierarchy. Proof of Proposition 5.4. In a first step the decay of the solutions at future null infinity will be deduced from the weighted energy inequality, and in a second step the continuation to the event horizon will be inferred from the redshift effect.

Step 1. The $p$-hierarchy consists of two steps which exploits (5-17) first with $p=2$, then with $p=1$; but in a zeroth step we need to obtain control on the angular derivatives from (5-17) with $p=1$ :

Since

$$
1-(n-1) \frac{2 m}{r^{n-2}}>\frac{1}{2} \quad(r>R),
$$

we have from the weighted energy inequality for $p=1$ on the domain $r_{0}^{\prime} \mathcal{D}_{\tau^{\prime}}^{\tau}$ for $\tau>\tau^{\prime} \geq \tau_{0} \doteq \frac{1}{2}\left(t_{0}-R^{*}\right)$,

$$
\begin{aligned}
\int_{\tau^{\prime}}^{\tau} \mathrm{d} u^{*} \int_{u^{*}+r_{0}^{\prime *}}^{\infty} \mathrm{d} v^{*} \int_{\mathbb{S}^{n-1}} \mathrm{~d} \mu_{\dot{\gamma}_{n-1}} & \frac{1}{4}|\not \nabla \psi|_{r^{2} \dot{\gamma}_{n-1}}^{2} \\
\leq\left(1-\frac{2 m}{R^{n-2}}\right)^{-1} & \left.\int_{\tau^{\prime}+r_{0}^{\prime *}}^{\infty} \mathrm{d} v^{*} \int_{\mathbb{S}^{n-1}} \mathrm{~d} \mu_{\dot{\gamma}_{n-1}} \frac{1}{2} r\left(\frac{\partial \psi}{\partial v^{*}}\right)^{2}\right|_{u^{*}=\tau^{\prime}} \\
& \quad+C(n, m, R)\left(1-\frac{2 m}{R^{n-2}}\right)^{-1} \int_{\Sigma_{\tau_{0}}}\left(J^{T}(\phi)+J^{T}(T \cdot \phi), n\right) ;
\end{aligned}
$$

here we have estimated the boundary integrals as follows. 
Choose $r_{0}^{\prime} \in\left(R^{*}, R^{*}+1\right)$ such that

$$
\begin{aligned}
& \int_{R^{*}}^{R^{*}+1} \mathrm{~d} r^{*} \int_{t_{0}+\left(r^{*}-R^{*}\right)}^{\infty} \mathrm{d} t \int_{\mathbb{S}^{n-1}} \mathrm{~d} \mu_{\dot{\gamma}_{n-1}}\left(1-\frac{2 m}{r^{n-2}}\right) r^{n-1} \\
& \times\left\{\frac{1}{r^{n}}\left(\frac{\partial \phi}{\partial r^{*}}\right)^{2}+\frac{1}{r^{n+1}}\left(\frac{\partial \phi}{\partial t}\right)^{2}+\frac{1}{r^{3}}\left(1-\frac{2 m}{r^{n-2}}\right)|\not \nabla \phi|_{r^{2} \dot{\gamma}_{n-1}}^{2}\right\} \\
& =\int_{t_{0}+\left(r_{0}^{\prime *}-R^{*}\right)}^{\infty} \mathrm{d} t \int_{\mathbb{S}^{n-1}} \mathrm{~d} \mu_{\dot{\gamma}_{n-1}}\left(1-\frac{2 m}{r_{0}^{\prime n-2}}\right) r_{0}^{\prime n-1} \times \\
& \times\left\{\frac{1}{r_{0}^{\prime n}}\left(\frac{\partial \phi}{\partial r^{*}}\right)^{2}+\frac{1}{r_{0}^{\prime n+1}}\left(\frac{\partial \phi}{\partial t}\right)^{2}+\frac{1}{r_{0}^{\prime 3}}\left(1-\frac{2 m}{r_{0}^{\prime n-2}}\right)|\nabla \phi|_{r^{2} \dot{\gamma}_{n-1}}^{2}\right\}
\end{aligned}
$$

then

$$
\begin{aligned}
& \left.\int_{2 \tau^{\prime}+r_{0}^{\prime}}^{2 \tau+r_{0}^{\prime *}} \mathrm{~d} t \int_{\mathbb{S}^{n-1}} \mathrm{~d} \mu_{\dot{\gamma}_{n-1}}\left[\frac{1}{4} r^{p}\left(\frac{\partial \psi}{\partial v^{*}}\right)^{2}+\frac{1}{4} r^{p}|\nabla \psi|_{r^{2} \dot{\gamma}_{n-1}}^{2}+\frac{n-1}{4} \frac{1}{2} r^{p}\left(\frac{n-3}{2}+\frac{n-1}{2} \frac{2 m}{r^{n-2}}\right) \frac{1}{r^{2}} \psi^{2}\right]\right|_{r=r_{0}^{\prime}} \\
& \leq \int_{2 \tau^{\prime}+r_{0}^{\prime *}}^{2 \tau+r_{0}^{\prime *}} \mathrm{~d} t \int_{\mathbb{S}^{n-1}} \mathrm{~d} \mu_{\dot{\gamma}_{n-1}} r_{0}^{\prime p-2}\left[\frac{1}{2}\left(\frac{n-1}{2}\right)^{2} \phi^{2}+\frac{1}{2} r_{0}^{\prime 2}\left(\frac{\partial \phi}{\partial v^{*}}\right)^{2}\right. \\
& \left.+\frac{1}{4} r_{0}^{\prime 2}|\not \phi|_{r^{2} \dot{\gamma}_{n-1}}^{2}+\frac{n-1}{4} \frac{1}{2}\left(\frac{n-3}{2}+\frac{n-1}{2} \frac{2 m}{R^{n-2}}\right) \phi^{2}\right]\left.\right|_{r=r_{0}^{\prime}} r^{n-1} \\
& \leq C(n, m, R) \int_{\Sigma_{\tau_{0}}}\left(J^{T}(\phi)+J^{T}(T \cdot \phi), n\right),
\end{aligned}
$$

because, by (4-23),

$$
\begin{array}{r}
\left.\int_{r_{0}^{\prime *}+2 \tau^{\prime}}^{r_{0}^{\prime *}+2 \tau} \mathrm{d} t \int_{\mathbb{S}^{n-1}} \mathrm{~d} \mu_{\dot{\gamma}_{n-1}} r^{n-1}\left[\frac{1}{4}\left(\frac{\partial \phi}{\partial v^{*}}\right)^{2}+\frac{n-1}{\left(4 r_{0}^{\prime}\right)^{2}}\left(1-\frac{2 m}{r^{n-2}}\right) \phi^{2}\right]\right|_{r=r_{0}^{\prime}} \\
\leq\left.\int_{r_{0}^{\prime *}+2 \tau^{\prime}}^{r_{0}^{\prime *}+2 \tau} \mathrm{d} t \int_{\mathbb{S}^{n-1}} \mathrm{~d} \mu_{\dot{\gamma}_{n-1}} r^{n-1}\left[\frac{1}{2}\left(1-\frac{2 m}{r^{n-2}}\right)|\not \phi|_{r^{2} \dot{\gamma}_{n-1}}^{2}+\frac{n-1}{2}\left(1-\frac{2 m}{r^{n-2}}\right)\left(\frac{\partial \phi}{\partial r^{*}}\right)^{2}\right]\right|_{r=r_{0}^{\prime}} \\
+C(n, m, R) \int_{\Sigma_{\tau_{0}}}\left(J^{T}(\phi), n\right),
\end{array}
$$

and by Proposition 4.1 (and the choice of $r_{0}^{\prime}$ ),

$$
\begin{array}{r}
\left.\int_{t_{0}+\left(r_{0}^{\prime *}-R^{*}\right)}^{\infty} \mathrm{d} t \int_{\mathbb{S}^{n-1}} \mathrm{~d} \mu_{\dot{\gamma}_{n-1}} r^{n-1}\left[\frac{1}{r_{0}^{\prime n}}\left(\frac{\partial \phi}{\partial r^{*}}\right)^{2}+\frac{1}{r_{0}^{\prime 3}}\left(1-\frac{2 m}{r_{0}^{\prime n-2}}\right)|\not \phi|_{r^{2} \dot{\gamma}_{n-1}}^{2}\right]\right|_{r=r_{0}^{\prime}} \\
\leq C(n, m) \int_{\Sigma_{\tau_{0}}}\left(J^{T}(\phi)+J^{T}(T \cdot \phi), n\right)
\end{array}
$$

Note that for the use of (4-23) that, with our choice of $R$,

$$
(n-3)+n \frac{2 m}{r^{n-2}}-(n-1)\left(\frac{2 m}{r^{n-2}}\right)^{2}>0 \quad(r>R) .
$$


$p=2:$ For $p=2,(5-17)$ reads

$$
\begin{aligned}
& \int_{\tau^{\prime}}^{\tau} \mathrm{d} u^{*} \int_{u^{*}+r_{0}^{\prime *}}^{\infty} \mathrm{d} v^{*} \int_{\mathbb{S}^{n-1}} \mathrm{~d} \mu_{\dot{\gamma}_{n-1}}\left[r\left(\frac{\partial \psi}{\partial v^{*}}\right)^{2}-\left.\frac{1}{2} r(n-2) \frac{2 m}{r^{n-2}}|\not \psi|\right|_{r^{2} \dot{\gamma}_{n-1}} ^{2}\right] \\
& \quad \leq\left.\left(1-\frac{2 m}{R^{n-2}}\right)^{-1} \int_{\tau^{\prime}+r_{0}^{\prime *}}^{\infty} \mathrm{d} v^{*} \int_{\mathbb{S}^{n-1}} \mathrm{~d} \mu_{\dot{\gamma}_{n-1}} \frac{1}{2} r^{2}\left(\frac{\partial \psi}{\partial v^{*}}\right)^{2}\right|_{u^{*}=\tau^{\prime}}+C(n, m, R) \int_{\Sigma_{\tau_{0}}}\left(J^{T}(\phi)+J^{T}(T \cdot \phi), n\right) .
\end{aligned}
$$

Thus, with the previous estimate (5-18),

$$
\begin{aligned}
& \int_{\tau^{\prime}}^{\tau} \mathrm{d} u^{*} \int_{u^{*}+r_{0}^{\prime}}^{\infty} \mathrm{d} v^{*} \int_{\mathbb{S}^{n-1}} \mathrm{~d} \mu_{\dot{\gamma}_{n-1}} r\left(\frac{\partial \psi}{\partial v^{*}}\right)^{2} \\
& \leq C(n, m, R)^{-1}\left\{\left.\int_{\tau^{\prime}+r_{0}^{\prime *}}^{\infty} \mathrm{d} v^{*} \int_{\mathbb{S}^{n-1}} \mathrm{~d} \mu_{\dot{\gamma}_{n-1}} \frac{1}{2} r^{2}\left(\frac{\partial \psi}{\partial v^{*}}\right)^{2}\right|_{u^{*}=\tau^{\prime}}+\int_{\Sigma_{\tau_{0}}}\left(J^{T}(\phi)+J^{T}(T \cdot \phi), n\right)\right\} .
\end{aligned}
$$

Let us define

$$
\tau_{j+1}=2 \tau_{j} \quad\left(j \in \mathbb{N}_{0}\right), \quad \tau_{0}=\frac{1}{2}\left(t_{0}-R^{*}\right) .
$$

Then there is a sequence $\left(\tau_{j}^{\prime}\right)_{j \in \mathbb{N}_{0}}$ with $\tau_{j}^{\prime} \in\left(\tau_{j}, \tau_{j+1}\right)\left(j \in \mathbb{N}_{0}\right)$ such that

$$
\begin{aligned}
& \left.\int_{\tau_{j}^{\prime}+r_{0}^{\prime *}}^{\infty} \mathrm{d} v^{*} \int_{\mathbb{S}^{n-1}} \mathrm{~d} \mu_{\dot{\gamma}_{n-1}} r\left(\frac{\partial \psi}{\partial v^{*}}\right)^{2}\right|_{u^{*}=\tau_{j}^{\prime}} \\
& \quad \leq \frac{1}{\tau_{j}} C(n, m, R)\left[\left.\int_{\tau_{j}+r_{0}^{\prime *}}^{\infty} \mathrm{d} v^{*} \int_{\mathbb{S}^{n-1}} \mathrm{~d} \mu_{\dot{\gamma}_{n-1}} r^{2}\left(\frac{\partial \psi}{\partial v^{*}}\right)^{2}\right|_{u^{*}=\tau_{j}}+\int_{\Sigma_{\tau_{0}}}\left(J^{T}(\phi)+J^{T}(T \cdot \phi), n\right)\right]
\end{aligned}
$$

and again by (5-17),

$$
\begin{aligned}
\int_{\tau_{j}+r_{0}^{\prime}}^{\infty} & \left.\mathrm{d} v^{*} \int_{\mathbb{S}^{n-1}} \mathrm{~d} \mu_{\dot{\gamma}_{n-1}} \frac{1}{2} r^{2}\left(\frac{\partial \psi}{\partial v^{*}}\right)^{2}\right|_{u^{*}=\tau_{j}} \\
& \leq C(n, m, R)\left[\left.\int_{\tau_{0}+r_{0}^{\prime *}}^{\infty} \mathrm{d} v^{*} \int_{\mathbb{S}^{n-1}} \mathrm{~d} \mu_{\dot{\gamma}_{n-1}} r^{2}\left(\frac{\partial \psi}{\partial v^{*}}\right)^{2}\right|_{u^{*}=\tau_{0}}+\int_{\Sigma_{\tau_{0}}}\left(J^{T}(\phi)+J^{T}(T \cdot \phi), n\right)\right] .
\end{aligned}
$$

Since $\frac{1}{\tau_{j}} \leq \frac{1}{\tau_{j}^{\prime}} \frac{\tau_{j+1}}{\tau_{j}}=\frac{2}{\tau_{j}^{\prime}}$, we have

$$
\begin{aligned}
& \left.\int_{\tau_{j}^{\prime}+r_{0}^{\prime *}}^{\infty} \mathrm{d} v^{*} \int_{\mathbb{S}^{n-1}} \mathrm{~d} \mu_{\dot{\gamma}_{n-1}} r\left(\frac{\partial \psi}{\partial v^{*}}\right)^{2}\right|_{u^{*}=\tau_{j}^{\prime}} \\
& \quad \leq \frac{C(n, m, R)}{\tau_{j}^{\prime}}\left[\left.\int_{\tau_{0}+r_{0}^{\prime *}}^{\infty} \mathrm{d} v^{*} \int_{\mathbb{S}^{n-1}} \mathrm{~d} \mu_{\dot{\gamma}_{n-1}} r^{2}\left(\frac{\partial \psi}{\partial v^{*}}\right)^{2}\right|_{u^{*}=\tau_{0}}+\int_{\Sigma_{\tau_{0}}}\left(J^{T}(\phi)+J^{T}(T \cdot \phi), n\right)\right] .
\end{aligned}
$$

$p=1:$ In order to deal with the timelike boundary integrals analogously to the above choose

$$
r_{j}^{\prime \prime *} \in\left(r_{0}^{\prime *}, r_{0}^{\prime *}+1\right)
$$


such that

$$
\begin{aligned}
& \left.\int_{2 \tau_{j}^{\prime}+r_{j}^{\prime \prime *}}^{2 \tau_{j+1}^{\prime}+r_{j}^{\prime \prime *}} \mathrm{~d} t \int_{\mathbb{S} n-1} \mathrm{~d} \mu_{\dot{\gamma}_{n-1}} r^{n-1}\left[\frac{1}{r^{n}}\left(\frac{\partial \phi}{\partial r^{*}}\right)^{2}+\frac{1}{r^{n+1}}\left(\frac{\partial \phi}{\partial t}\right)^{2}+\frac{1}{r^{3}}\left(1-\frac{2 m}{r^{n-2}}\right)|\nabla \phi|_{r^{2} \dot{\gamma}_{n-1}}^{2}\right]\right|_{r=r_{j}^{\prime \prime}} \\
& \leq C(n, m) \int_{\Sigma_{\tau_{j}^{\prime}}}\left(J^{T}(\phi)+J^{T}(T \cdot \phi), n\right) .
\end{aligned}
$$

Then, proceeding as before,

$$
\begin{array}{r}
\left.\left.\int_{2 \tau_{j}^{\prime}+r_{j}^{\prime \prime *}}^{2 \tau_{j+1}^{\prime}+r_{j}^{\prime \prime *} \mathrm{~d} t \int_{\mathbb{S}^{n-1}} \mathrm{~d} \mu_{\dot{\gamma}_{n-1}}\left[\frac{1}{4} r\left(\frac{\partial \psi}{\partial v^{*}}\right)^{2}+\frac{1}{4} r \mid \not \psi\right.} \psi\right|_{r^{2} \dot{\gamma}_{n-1}} ^{2}+\frac{n-1}{4} \frac{1}{2} r\left(\frac{n-3}{2}+\frac{n-1}{2} \frac{2 m}{r^{n-2}}\right) \frac{1}{r^{2}} \psi^{2}\right]\left.\right|_{r=r_{j}^{\prime \prime}} \\
\leq C(n, m, R) \int_{\Sigma_{\tau_{j}^{\prime}}}\left(J^{T}(\phi)+J^{T}(T \cdot \phi), n\right)
\end{array}
$$

Now apply (5-17) to the region ${ }_{j}^{\prime \prime} \mathcal{D}_{\tau_{j}^{\prime}}^{\tau_{j+1}^{\prime}}$ to obtain

$$
\begin{aligned}
& \int_{\tau_{j}^{\prime}}^{\tau_{j+1}^{\prime}} \mathrm{d} u^{*} \int_{u^{*}+r_{j}^{\prime \prime}}^{\infty} \mathrm{d} v^{*} \int_{\mathbb{S}^{n-1}} \mathrm{~d} \mu_{\dot{\gamma}_{n-1}} \times\left[\frac{1}{2}\left(\frac{\partial \psi}{\partial v^{*}}\right)^{2}+\frac{1}{4}|\nabla \psi|_{r^{2} \dot{\gamma}_{n-1}}^{2}\right] \\
& \leq\left.\left(1-\frac{2 m}{R^{n-2}}\right)^{-1} \int_{\tau_{j}^{\prime}+r_{j}^{\prime \prime *}}^{\infty} \mathrm{d} v^{*} \int_{\mathbb{S}^{n-1}} \mathrm{~d} \mu_{\dot{\gamma}_{n-1}} \frac{1}{2} r\left(\frac{\partial \psi}{\partial v^{*}}\right)^{2}\right|_{u^{*}=\tau_{j}^{\prime}}+C(n, m, R) \int_{\Sigma_{\tau_{j}^{\prime}}}\left(J^{T}(\phi)+J^{T}(T \cdot \phi), n\right) .
\end{aligned}
$$

By virtue of the result (5-20) from the case $p=2$, this yields

$$
\begin{aligned}
& \int_{\tau_{j}^{\prime}}^{\tau_{j+1}^{\prime}} \mathrm{d} u^{*} \int_{u^{*}+r_{j}^{\prime \prime}}^{\infty} \mathrm{d} v^{*} \int_{\mathbb{S}^{n-1}} \mathrm{~d} \mu_{\dot{\gamma}_{n-1}}\left[\frac{1}{2}\left(\frac{\partial \psi}{\partial v^{*}}\right)^{2}+\frac{1}{4}|\nabla \psi|_{r^{2} \dot{\gamma}_{n-1}}^{2}\right] \\
& \leq \frac{C(n, m, R)}{\tau_{j}^{\prime}}\left[\left.\int_{\tau_{0}+r_{0}^{\prime *}}^{\infty} \mathrm{d} v^{*} \int_{\mathbb{S}^{n-1}} \mathrm{~d} \mu_{\dot{\gamma}_{n-1}} r^{2}\left(\frac{\partial \psi}{\partial v^{*}}\right)^{2}\right|_{u^{*}=\tau_{0}}+\int_{\Sigma_{\tau_{0}}}\left(J^{T}(\phi)+J^{T}(T \cdot \phi), n\right)\right] \\
& +C(n, m, R) \int_{\Sigma_{\tau_{j}^{\prime}}}\left(J^{T}(\phi)+J^{T}(T \cdot \phi), n\right) .
\end{aligned}
$$

Step 2. Our aim is to prove decay for the nondegenerate energy. Let us first find an estimate for

$$
\int_{\tau_{j}^{\prime}}^{\tau_{j+1}^{\prime}} \mathrm{d} \tau \int_{\Sigma_{\tau}}\left(J^{N}(\phi), n\right)=\int_{\tau_{j}^{\prime}}^{\tau_{j+1}^{\prime}} \mathrm{d} \tau \int_{\Sigma_{\tau} \cap\left\{r \leq r_{j}^{\prime \prime}\right\}}\left(J^{N}(\phi), n\right)+\int_{\tau_{j}^{\prime}}^{\tau_{j+1}^{\prime}} \mathrm{d} \tau \int_{\Sigma_{\tau} \cap\left\{r \geq r_{j}^{\prime \prime}\right\}}\left(J^{T}(\phi), n\right) .
$$

The estimate of the first term is exactly the content of Corollary 4.3, and for the second term

$$
\begin{aligned}
\int_{\tau_{j}^{\prime}}^{\left.\tau_{j+1}^{\prime} \mathrm{d} \tau \int_{\Sigma_{\tau}^{\prime} \cap\{r} \geq r_{j}^{\prime \prime}\right\}} & \left(J^{T}(\phi), n\right) \\
& =\int_{\tau_{j}^{\prime}}^{\tau_{j+1}^{\prime}} \mathrm{d} u^{*} \int_{u^{*}+r_{j}^{\prime \prime}}^{\infty} \mathrm{d} v^{*} \int_{\mathbb{S}^{n-1}} \mathrm{~d} \mu_{\dot{\gamma}_{n-1}} r^{n-1}\left[\frac{1}{2}\left(\frac{\partial \phi}{\partial v^{*}}\right)^{2}+\frac{1}{2}\left(1-\frac{2 m}{r^{n-2}}\right)|\nabla \phi|_{r^{2} \dot{\gamma}_{n-1}}^{2}\right]
\end{aligned}
$$


we can use (5-22) once we have turned it into an estimate for the derivatives of $\phi$. Note that

$$
\begin{aligned}
\int_{u^{*}+r_{0}^{\prime *}}^{\infty} \mathrm{d} v^{*}\left(\frac{\partial \psi}{\partial v^{*}}\right)^{2} & =\int_{u^{*}+r_{0}^{\prime *}}^{\infty} \mathrm{d} v^{*}\left[\frac{n-1}{2}\left(1-\frac{2 m}{r^{n-2}}\right) r^{\frac{n-3}{2}} \frac{\partial}{\partial v^{*}}\left(r^{\frac{n-1}{2}} \phi^{2}\right)+r^{n-1}\left(\frac{\partial \phi}{\partial v^{*}}\right)^{2}\right] \\
& =-\left.\frac{n-1}{2}\left(1-\frac{2 m}{r^{n-2}}\right) r^{n-2} \phi^{2}\right|_{v^{*}=u^{*}+r_{0}^{\prime *}} \\
& +\int_{u^{*}+r_{0}^{\prime *}}^{\infty} \mathrm{d} v^{*}\left\{-\frac{n-1}{2}\left(1-\frac{2 m}{r^{n-2}}\right)\left[(n-2) \frac{2 m}{r^{n}}+\frac{n-3}{2}\left(1-\frac{2 m}{r^{n-2}}\right) \frac{1}{r^{2}}\right] \phi^{2}+\left(\frac{\partial \phi}{\partial v^{*}}\right)^{2}\right\} r^{n-1}
\end{aligned}
$$

and, by Lemma C.2,

$$
\int_{u^{*}+r_{0}^{\prime *}}^{\infty} \mathrm{d} v^{*} \frac{1}{r^{2}} \phi^{2} r^{n-1} \leq C(n, m) \int_{u^{*}+r_{0}^{\prime *}}^{\infty} \mathrm{d} v^{*}\left(\frac{\partial \psi}{\partial v^{*}}\right)^{2}+\left.C(n, m) r^{n-1} \phi^{2}\right|_{\left(u^{*}, v^{*}=u^{*}+r_{0}^{\prime *}\right)} .
$$

Thus

$$
\int_{u^{*}+r_{0}^{\prime *}}^{\infty} \mathrm{d} v^{*}\left(\frac{\partial \phi}{\partial v^{*}}\right)^{2} r^{n-1} \leq C(n, m, R)\left[\left.\phi^{2}\right|_{\left(u^{*}, u^{*}+r_{0}^{\prime *}\right)}+\int_{u^{*}+r_{0}^{\prime *}}^{\infty} \mathrm{d} v^{*}\left(\frac{\partial \psi}{\partial v^{*}}\right)^{2}\right],
$$

and finally, in view of (5-21),

$$
\begin{aligned}
& \int_{\tau_{j}^{\prime}}^{\tau_{j+1}^{\prime}} \mathrm{d} u^{*} \int_{u^{*}+r_{j}^{\prime \prime *}}^{\infty} \mathrm{d} v^{*} \int_{\mathbb{S}^{n-1}} \mathrm{~d} \mu_{\gamma_{n-1}}\left(\frac{\partial \phi}{\partial v^{*}}\right)^{2} r^{n-1} \\
& \leq C(n, m, R) \int_{\tau_{j}^{\prime}}^{\tau_{j+1}^{\prime}} \mathrm{d} u^{*} \int_{u^{*}+r_{j}^{\prime \prime *}}^{\infty} \mathrm{d} v^{*} \int_{\mathbb{S}^{n-1}} \mathrm{~d} \mu_{\dot{\gamma}_{n-1}}\left(\frac{\partial \psi}{\partial v^{*}}\right)^{2}+C(n, m, R) \int_{\Sigma_{\tau_{j}^{\prime}}}\left(J^{T}(\phi)+J^{T}(T \cdot \phi), n\right) .
\end{aligned}
$$

Therefore, putting the estimates for the two terms back together,

$$
\begin{aligned}
& \int_{\tau_{j}^{\prime}}^{\tau_{j+1}^{\prime}} \mathrm{d} \tau \int_{\Sigma_{\tau}}\left(J^{N}(\phi), n\right) \\
& \leq C(n, m, R) \int_{\tau_{j}^{\prime}}^{\tau_{j+1}^{\prime}} \mathrm{d} u^{*} \int_{u^{*}+r_{j}^{\prime \prime}}^{\infty} \mathrm{d} v^{*} \int_{\mathbb{S}^{n-1}} \mathrm{~d} \mu_{\dot{\gamma}_{n-1}}\left\{\left(\frac{\partial \psi}{\partial v^{*}}\right)^{2}+|\not \psi|_{r^{2} \dot{\gamma}_{n-1}}^{2}\right\} \\
& \quad+C(n, m) \int_{\Sigma_{\tau_{j}^{\prime}}}\left(J^{N}(\phi)+J^{T}(T \cdot \phi), n\right) \\
& \leq \frac{C(n, m, R)}{\tau_{j}^{\prime}}\left[\left.\int_{\tau_{0}+r_{0}^{\prime *}}^{\infty} \mathrm{d} v^{*} \int_{\mathbb{S}^{n-1}} \mathrm{~d} \mu_{\dot{\gamma}_{n-1}} r^{2}\left(\frac{\partial \psi}{\partial v^{*}}\right)^{2}\right|_{u^{*}=\tau_{0}}+\int_{\Sigma_{\tau_{0}}}\left(J^{T}(\phi)+J^{T}(T \cdot \phi), n\right)\right] \\
& \quad+C(n, m, R) \int_{\Sigma_{\tau_{j}^{\prime}}}\left(J^{N}(\phi)+J^{T}(T \cdot \phi), n\right),
\end{aligned}
$$

where we have now used (5-22). The same inequality holds for $\tau_{j+2}^{\prime}$ in place of $\tau_{j+1}^{\prime}$, by adding the inequalities corresponding to the intervals $\left[\tau_{j}^{\prime}, \tau_{j+1}^{\prime}\right]$ and $\left[\tau_{j+1}^{\prime}, \tau_{j+2}^{\prime}\right]$ and using Proposition 5.2 for the 
last term. So there is a sequence

$$
\left(\tau_{j}^{\prime \prime}\right)_{j \in \mathbb{N}}, \quad \tau_{j}^{\prime \prime} \in\left(\tau_{j}^{\prime}, \tau_{j+2}^{\prime}\right)
$$

such that

$$
\int_{\tau_{j}^{\prime}}^{\tau_{j+2}^{\prime}} \mathrm{d} \tau \int_{\Sigma_{\tau}}\left(J^{N}(\phi), n\right) \geq \tau_{j+1} \int_{\Sigma_{\tau_{j}^{\prime \prime}}}\left(J^{N}(\phi), n\right),
$$

and since $\frac{1}{\tau_{j+1}} \leq \frac{1}{\tau_{j}^{\prime \prime}} \frac{\tau_{j+3}}{\tau_{j+1}}=\frac{4}{\tau_{j}^{\prime \prime}}$, we have

$$
\int_{\Sigma_{\tau_{j}^{\prime \prime}}}\left(J^{N}(\phi), n\right) \leq \frac{4}{\tau_{j}^{\prime \prime}} \int_{\tau_{j}}^{\tau_{j+2}^{\prime}} \mathrm{d} \tau \int_{\Sigma_{\tau}^{\prime}}\left(J^{N}(\phi), n\right)
$$

Now for any given $\tau>\tau_{0}$ we may choose

$$
j^{*}=\max \left\{j \in \mathbb{N}: \tau_{j}^{\prime \prime} \leq \tau\right\}
$$

so that, by (5-1),

$$
\int_{\Sigma_{\tau}}\left(J^{N}(\phi), n\right) \leq C \int_{\Sigma_{\tau_{j *}^{\prime \prime}}}\left(J^{N}(\phi), n\right)
$$

with $\frac{\tau}{\tau_{j^{*}}^{\prime \prime}} \leq \frac{\tau_{j^{*}+1}^{\prime \prime}}{\tau_{j^{*}}^{\prime \prime}} \leq 2^{4}$. In particular we may estimate the last integral in (5-24),

$$
\int_{\Sigma_{\tau_{j}^{\prime}}}\left(J^{N}(\phi)+J^{T}(T \cdot \phi), n\right) \leq \frac{C}{\tau_{j}^{\prime}} \int_{\tau_{j-1}^{\prime}}^{\tau_{j+1}^{\prime}} \mathrm{d} \tau \int_{\Sigma_{\tau}}\left(J^{N}(\phi)+J^{N}(T \cdot \phi), n\right),
$$

to see that in fact we have

$$
\begin{array}{r}
\int_{\tau_{j}^{\prime}}^{\tau_{j+2}^{\prime}} \mathrm{d} \tau \int_{\Sigma_{\tau}}\left(J^{N}(\phi), n\right) \leq \frac{C(n, m, R)}{\tau_{j}^{\prime}}\left[\int_{\tau_{0}+r_{0}^{\prime *}}^{\infty} \mathrm{d} v^{*} \int_{\mathbb{S}^{n-1}} \mathrm{~d} \mu_{\dot{\gamma}_{n-1}}\left\{r^{2}\left(\frac{\partial r^{\frac{n-1}{2}} \phi}{\partial v^{*}}\right)^{2}+r^{2}\left(\frac{\partial r^{\frac{n-1}{2}} \frac{\partial \phi}{\partial t}}{\partial v^{*}}\right)\right\}\right. \\
\left.+\int_{\Sigma_{\tau_{0}}}\left(J^{N}(\phi)+J^{N}(T \cdot \phi)+J^{T}\left(T^{2} \phi\right), n\right)\right] .
\end{array}
$$

Again, with the sequence $\left(\tau_{j}^{\prime \prime}\right)_{j \in \mathbb{N}}$,

$$
\int_{\Sigma_{\tau_{j}^{\prime \prime}}}\left(J^{N}(\phi), n\right) \leq \frac{1}{\tau_{j+1}} \int_{\tau_{j}^{\prime}}^{\tau_{j+2}^{\prime}} \mathrm{d} \tau \int_{\Sigma_{\tau}}\left(J^{N}(\phi), n\right)
$$

and since $\frac{1}{\tau_{j+1}} \frac{1}{\tau_{j}^{\prime}} \leq \frac{2^{5}}{\tau_{j}^{\prime \prime 2}}$ we obtain by virtue of Proposition 5.2 our final result:

$$
\begin{array}{r}
\int_{\Sigma_{\tau}}\left(J^{N}(\phi), n\right) \leq \frac{C(n, m, R)}{\tau^{2}}\left[\int_{\tau_{0}+R^{*}}^{\infty} \mathrm{d} v^{*} \int_{\mathbb{S}^{n-1}} \mathrm{~d} \mu_{\dot{\gamma}_{n-1}}\left\{r^{2}\left(\frac{\partial r^{\frac{n-1}{2} \phi}}{\partial v^{*}}\right)^{2}+r^{2}\left(\frac{\partial r^{\frac{n-1}{2}} \frac{\partial \phi}{\partial t}}{\partial v^{*}}\right)^{2}\right\}\right. \\
\left.+\int_{\Sigma_{\tau_{0}}}\left(J^{N}(\phi)+J^{N}(T \cdot \phi)+J^{T}\left(T^{2} \cdot \phi\right), n\right)\right] .
\end{array}
$$


5C. Improved interior decay of the first-order energy. In this section we prove an energy estimate for the first-order energy which improves the decay rate as compared to Proposition 5.4 in a bounded radial region.

Remark 5.5. The argument largely depends on the asymptotic properties of the spacetime, and is similar and slightly easier in Minkowski space [Schlue 2012].

Proposition 5.6 (improved interior first-order energy decay). Let $0<\delta<\frac{1}{2}, R>\sqrt[n-2]{\frac{8 n m}{\delta}}$, and let $\phi$ be a solution of the wave equation (1-1) with initial data on $\Sigma_{\tau_{1}}\left(\tau_{1}>0\right)$ satisfying

$$
\begin{aligned}
D \doteq \int_{\tau_{1}+R^{*}}^{\infty} \mathrm{d} v^{*} \int_{\mathbb{S}^{n-1}} \mathrm{~d} \mu_{\dot{\gamma}_{n-1}} & \left\{\sum_{k=0}^{1} r^{4-\delta}\left(\frac{\partial\left(T^{k} \cdot \chi\right)}{\partial v^{*}}\right)^{2}\right. \\
& \left.+\sum_{k=0}^{4} r^{2}\left(\frac{\partial\left(T^{k} \cdot \psi\right)}{\partial v^{*}}\right)^{2}+\sum_{k=0}^{3} \sum_{i=1}^{\frac{n(n-1)}{2}} r^{2}\left(\frac{\partial T^{k} \Omega_{i} \psi}{\partial v^{*}}\right)^{2}\right\}\left.\right|_{u^{*}=\tau_{1}} \\
& +\int_{\Sigma_{\tau_{1}}}\left(\sum_{k=0}^{5} J^{N}\left(T^{k} \cdot \phi\right)+\sum_{k=0}^{4} \sum_{i=1}^{\frac{n(n-1)}{2}} J^{N}\left(T^{k} \Omega_{i} \phi\right), n\right)<\infty
\end{aligned}
$$

Then there exists a constant $C(n, m, \delta, R)$ such that

$$
\int_{\Sigma_{\tau}^{\prime}}\left(J^{N}(T \cdot \phi), n\right) \leq \frac{C D}{\tau^{4-2 \delta}} \quad\left(\tau>\tau_{1}\right)
$$

where $\Sigma_{\tau}^{\prime}=\Sigma_{\tau} \cap\{r \leq R\}$.

In addition to the weighted energy identity arising from the multiplier $r^{p} \frac{\partial}{\partial v^{*}}$ that was used to prove Proposition 5.4, we will here also use a commutation with $\frac{\partial}{\partial v^{*}}$ to obtain the energy decay for $\frac{\partial \phi}{\partial t}$ of Proposition 5.6.

Weighted energy and commutation. Consider the current

$$
\stackrel{v}{J}_{\mu}(\phi) \doteq T_{\mu \nu}(\chi) V^{v},
$$

where now

$$
\chi \doteq \partial_{v^{*}} \psi=\frac{\partial\left(r^{\frac{n-1}{2}} \phi\right)}{\partial v^{*}}, \quad V=r^{q} \frac{\partial}{\partial v^{*}}, \quad q=p-(n-1), \quad 2<p<4, \quad \delta=4-p .
$$

Notation. To make the dependence on $p$ explicit, we define

$$
\stackrel{v}{K}_{p}(\phi) \doteq \nabla^{\mu} \stackrel{v}{J}_{\mu}(\phi) .
$$


The error terms for $\stackrel{v}{K}$ arise from the fact that $\chi$ is not a solution of (1-1); here, similarly to (5-11), we find

$$
\begin{aligned}
\square_{g} \chi=-\frac{n-1}{2 r^{3}}\{(n-3) & \left.+n \frac{2 m}{r^{n-2}}-(n-1)^{2}\left(\frac{2 m}{r^{n-2}}\right)^{2}\right\} \psi \\
+ & \frac{n-1}{4 r^{2}}\left[(n-3)+(n-1) \frac{2 m}{r^{n-2}}\right] \chi+\frac{1}{r}\left[2-n \frac{2 m}{r^{n-2}}\right] \varangle \psi+\frac{n-1}{r} \frac{\partial \chi}{\partial r^{*}} .
\end{aligned}
$$

Hence

$$
\begin{aligned}
\stackrel{v}{K}_{p}(\phi)= & \square(\chi) V \cdot \chi+K^{V}(\chi) \\
= & \frac{1}{2} p r^{q-1}\left(\frac{\partial \chi}{\partial v^{*}}\right)^{2}+\frac{1}{2}\left[(2-p)-(n-p) \frac{2 m}{r^{n-2}}\right] r^{q-1}|\nabla \chi|^{2} \\
& -\frac{n-1}{2} r^{q-3}\left[(n-3)+n \frac{2 m}{r^{n-2}}-(n-1)^{2}\left(\frac{2 m}{r^{n-2}}\right)^{2}\right] \psi \frac{\partial^{2} \psi}{\partial v^{* 2}} \\
& +\frac{n-1}{8} r^{q-2}\left[(n-3)+(n-1) \frac{2 m}{r^{n-2}}\right] \frac{\partial \chi^{2}}{\partial v^{*}} \\
& +r^{q-1}\left[2-n \frac{2 m}{r^{n-2}}\right]\left(\not \psi \psi\left(\frac{\partial \chi}{\partial v^{*}}\right),\right.
\end{aligned}
$$

which is not positive definite. However, we have

$$
\begin{aligned}
\frac{1}{4} p r^{p-1}\left(\frac{\partial \chi}{\partial v^{*}}\right)^{2} \leq & { }^{v} \\
p & (\phi) \cdot r^{n-1}+\frac{1}{2}\left[(p-2)+(n-p) \frac{2 m}{r^{n-2}}\right] r^{p-1}|\not \chi|^{2} \\
& +\frac{(n-1)^{2}(n-2)^{2}}{2} r^{(p-2)-1} \frac{1}{r^{2}} \psi^{2}+\frac{4}{p} r^{(p-2)-1} r^{2}(\not \psi)^{2} \\
& -\frac{n-1}{8} r^{p-2}\left[(n-3)+(n-1) \frac{2 m}{r^{n-2}}\right] \frac{\partial \chi^{2}}{\partial v^{*}},
\end{aligned}
$$

where we have used that

$$
n-2>n-3+n \frac{2 m}{r^{n-2}}-(n-1)^{2}\left(\frac{2 m}{r^{n-2}}\right)^{2} \geq n-3
$$

(is decreasing) on $r>\sqrt[n-2]{4 n m}$. The key insight here is that we are able to control all other terms on the right-hand side of (5-38) by the current $J$ of Section $5 \mathrm{~B}$ with $p-2$ in the role of $p$; i.e.,

$$
\begin{aligned}
& \int_{\tau_{1}}^{\tau_{2}} \mathrm{~d} u^{*} \int_{u^{*}+R^{*}}^{\infty} \mathrm{d} v^{*} \int_{\mathbb{S}^{n-1}} \mathrm{~d} \mu_{\dot{\gamma}_{n-1}} r^{p-1}\left(\frac{\partial \chi}{\partial v^{*}}\right)^{2} \\
& \leq C(n, m, \delta, p, R) \int_{R_{\mathcal{D}} \tau_{1}}\left\{\stackrel{v}{K}_{p}^{v}(\phi)+\stackrel{r}{K}_{p-2}^{\frac{n(n-1)}{2}} \stackrel{r}{K}_{p-2}\left(\Omega_{i} \phi\right)\right\} \\
& \quad+\left.C(n, m, \delta, p, R) \int_{i=1}^{2 \tau_{2}+R^{*}} \mathrm{~d} t \int_{\tau_{1}+R^{*}} \mathrm{~d} \mu_{\dot{\gamma}_{n-1}}\left\{\psi^{2}+\left(\frac{\partial \psi}{\partial v^{*}}\right)^{2}+\sum_{i=1}^{\frac{n(n-1)}{2}}\left(\Omega_{i} \psi\right)^{2}+\sum_{i=1}^{\frac{n(n-1)}{2}}\left|\not \Omega_{i} \psi\right|^{2}\right\}\right|_{r=R}
\end{aligned}
$$


Indeed, the first term $\left|\nabla \partial_{v^{*}} \psi\right|^{2}$ can be integrated by parts twice (such that we can absorb the resulting $\partial_{v^{*}} \chi$ term in the left-hand side):

$$
\begin{aligned}
& \int_{\tau_{1}}^{\tau_{2}} \mathrm{~d} u^{*} \int_{u^{*}+R^{*}}^{\infty} \mathrm{d} v^{*} \int_{\mathbb{S}^{n-1}} \mathrm{~d} \mu_{\dot{\gamma}_{n-1}} r^{p-1}|\not \nabla \chi|^{2} \\
& =-\int_{\tau_{1}}^{\tau_{2}} \mathrm{~d} u^{*} \int_{u^{*}+R^{*}}^{\infty} \mathrm{d} v^{*} \int_{\mathbb{S}^{n-1}} \mathrm{~d} \mu_{\dot{\gamma}_{n-1}} r^{p-1} \not \partial \partial_{v^{*}} \psi \cdot \partial_{v^{*}} \psi \\
& =-\left.\int_{\tau_{1}}^{\tau_{2}} \mathrm{~d} u^{*} \int_{\mathbb{S}^{n-1}} \mathrm{~d} \mu_{\dot{\gamma}_{n-1}} r^{p-1} \not \psi \frac{\partial \psi}{\partial v^{*}}\right|_{u^{*}+R^{*}} ^{\infty} \\
& +\int_{\tau_{1}}^{\tau_{2}} \mathrm{~d} u^{*} \int_{u^{*}+R^{*}}^{\infty} \mathrm{d} v^{*} \int_{\mathbb{S}^{n-1}} \mathrm{~d} \mu_{\dot{\gamma}_{n-1}}\left\{(p-1) r^{p-2}\left(1-\frac{2 m}{r^{n-2}}\right)(\Delta \psi)\left(\frac{\partial \psi}{\partial v^{*}}\right)\right. \\
& \left.+r^{p-1}(\not \psi) \frac{\partial \chi}{\partial v^{*}}+r^{p-1} \frac{2}{r}\left(1-\frac{2 m}{r^{n-2}}\right)(\not \psi) \frac{\partial \psi}{\partial v^{*}}\right\} \\
& \leq\left.\int_{\tau_{1}}^{\tau_{2}} \mathrm{~d} u^{*} \int_{\mathbb{S}^{n-1}} \mathrm{~d} \mu_{\dot{\gamma}_{n-1}} r^{p-1}(\not \psi \psi)\left(\frac{\partial \psi}{\partial v^{*}}\right)\right|_{v^{*}=u^{*}+R^{*}} \\
& +\int_{\tau_{1}}^{\tau_{2}} \mathrm{~d} u^{*} \int_{u^{*}+R^{*}}^{\infty} \mathrm{d} v^{*} \int_{\mathbb{S}^{n-1}} \mathrm{~d} \mu_{\dot{\gamma}_{n-1}}\left\{\left(p-1+\frac{n-2}{p}+2\right) r^{(p-2)-1}(\not \psi)^{2} r^{2}\right. \\
& \left.+(p-1+2) r^{(p-2)-1}\left(\frac{\partial \psi}{\partial v^{*}}\right)^{2}+\frac{1}{2} \frac{p}{4} \frac{2}{n-2} r^{p-1}\left(\frac{\partial \chi}{\partial v^{*}}\right)^{2}\right\} .
\end{aligned}
$$

The second term in (5-38) is controlled by the Hardy inequality

$$
\begin{aligned}
& \frac{1}{2} \int_{u^{*}+R^{*}}^{\infty} \mathrm{d} v^{*} r^{(p-2)-1} \frac{1}{r^{2}} \psi^{2} \\
& \quad \leq\left.\frac{1}{4-p} \frac{1}{R^{4-p}} \frac{1}{1-\frac{2 m}{R^{n-2}}} \psi^{2}\right|_{\left(u^{*}, u^{*}+R^{*}\right)}+\frac{2}{(4-p)^{2}\left(1-\frac{2 m}{R^{n-2}}\right)^{2}} \int_{u^{*}+R^{*}}^{\infty} \mathrm{d} v^{*} r^{(p-2)-1}\left(\frac{\partial \psi}{\partial v^{*}}\right)^{2},
\end{aligned}
$$

and the third term simply by the following commutation with $\Omega_{i}$ :

Lemma 5.7. For any function $\phi \in \mathrm{H}^{2}\left(S_{r}\right)$ we have $\phi_{r^{2} \hat{\gamma}_{n-1}} \phi \in \mathrm{L}^{2}\left(S_{r}\right)$, and there exists a constant $C>0$ such that

$$
\int_{\mathbb{S}^{n-1}}(\Delta \psi)^{2} r^{2} \mathrm{~d} \mu_{\dot{\gamma}_{n-1}} \leq C \int_{\mathbb{S}^{n-1}}\left\{\sum_{i=1}^{\frac{n(n-1)}{2}}\left|\not \nabla\left(\Omega_{i} \psi\right)\right|^{2}+|\not \nabla \psi|^{2}\right\} \mathrm{d} \mu_{\dot{\gamma}_{n-1}} .
$$

The last term in (5-38) we can rearrange as follows:

$$
\begin{aligned}
-\frac{n-1}{8} r^{p-2}\left[n-3+(n-1) \frac{2 m}{r^{n-2}}\right] \frac{\partial \chi^{2}}{\partial v^{*}} & \\
= & -\frac{\partial}{\partial v^{*}}\left\{\frac{n-1}{8} r^{p-2}\left[(n-3)+(n-1) \frac{2 m}{r^{n-2}}\right]\left(\frac{\partial \psi}{\partial v^{*}}\right)^{2}\right\} \\
& +\frac{n-1}{8} r^{(p-2)-1}\left[(p-2)(n-3)+(n-1)((p-2)+(n-2)) \frac{2 m}{r^{n-2}}\right]\left(1-\frac{2 m}{r^{n-2}}\right)\left(\frac{\partial \psi}{\partial v^{*}}\right)^{2} .
\end{aligned}
$$


Therefore (see also Appendix B),

$$
\begin{aligned}
\int_{\tau_{1}}^{\tau_{2}} \mathrm{~d} u^{*} \int_{u^{*}+R^{*}}^{\infty} \mathrm{d} v^{*} \int_{\mathbb{S}^{n-1}} \mathrm{~d} \mu_{\dot{\gamma}_{n-1}} \frac{1}{8} p r^{p-1}\left(\frac{\partial \chi}{\partial v^{*}}\right)^{2} \\
\leq \frac{1}{2} \frac{1}{1-\frac{2 m}{R^{n-2}}} \int_{R_{\mathcal{D}_{\tau}}^{\tau_{2}}} K_{p}^{v}(\phi) \mathrm{d} \mu_{g} \\
\quad+\left.C(n, p, \delta, R) \int_{\tau_{1}}^{\tau_{2}} \mathrm{~d} u^{*} \int_{\mathbb{S}^{n-1}} \mathrm{~d} \mu_{\dot{\gamma}_{n-1}}\left\{r^{p-2}(\not \psi)^{2} r^{2}+r^{p-2}\left(\frac{\partial \psi}{\partial v^{*}}\right)^{2}+\psi^{2}\right\}\right|_{v^{*}=u^{*}+R^{*}} \\
+C(p, n, \delta, R) \int_{\tau_{1}}^{\tau_{2}} \mathrm{~d} u^{*} \int_{u^{*}+R^{*}}^{\infty} \int_{\mathbb{S}^{n-1}} \mathrm{~d} \mu_{\dot{\gamma}_{n-1}} \\
\quad+\frac{n-1}{8} \int_{\tau_{1}}^{\tau_{2}} \mathrm{~d} u^{*} \int_{u^{*}+R^{*}}^{\infty} \mathrm{d} v^{*} \int_{\mathbb{S}^{n-1}} \mathrm{~d} \mu_{\dot{\gamma}_{n-1}} r^{p-2}\left[n+3+(n-1) \frac{2 m}{r^{n-2}}\right] \frac{\partial \chi^{2}}{\partial v^{*}} .
\end{aligned}
$$

Now, recall (5-15), and note that

$$
\delta-(n-(2-\delta)) \frac{2 m}{r^{n-2}}>\frac{\delta}{2} \quad\left(r>\sqrt[n-2]{\frac{4 n m}{\delta}}\right),
$$

to see that

$$
\begin{aligned}
& r^{(p-2)-1} \sum_{i=1}^{\frac{n(n-1)}{2}}\left|\not r^{\frac{n-1}{2}} \Omega_{i} \phi\right|^{2} \\
& \quad \leq \frac{4}{\delta} \sum_{i=1}^{\frac{n(n-1)}{2}} \stackrel{r}{K}_{p-2}\left(\Omega_{i} \phi\right) r^{n-1}-\frac{4}{\delta} \sum_{i=1}^{\frac{n(n-1)}{2}} \frac{n-1}{4}\left[\frac{n-3}{2}+\frac{n-1}{2} \frac{2 m}{r^{n-2}}\right] r^{(p-2)-2} \frac{\partial\left(r^{\frac{n-1}{2}} \Omega_{i} \phi\right)^{2}}{\partial v^{*}} .
\end{aligned}
$$

So

$$
\begin{aligned}
& \int_{\tau_{1}}^{\tau_{2}} \mathrm{~d} u^{*} \int_{u^{*}+R^{*}}^{\infty} \mathrm{d} v^{*} \int_{\mathbb{S}^{n-1}} \mathrm{~d} \mu_{\dot{\gamma}_{n-1}} p r^{p-1}\left(\frac{\partial \chi}{\partial v^{*}}\right)^{2} \\
& \leq C(n, m, \delta, p, R) \int_{R_{\mathcal{D}} \tau_{2}}\left\{\stackrel{v}{K}_{p}(\phi)+\stackrel{r}{K}_{p-2}(\phi)+\sum_{i=1}^{\frac{n(n-1)}{2}} \stackrel{r}{K}_{p-2}\left(\Omega_{i} \phi\right)\right\} \\
& -C(n, m, \delta, p) \int_{\tau_{1}}^{\tau_{2}} \mathrm{~d} u^{*} \int_{u^{*}+R^{*}}^{\infty} \mathrm{d} v^{*} \int_{\mathbb{S}^{n-1}} \mathrm{~d} \mu_{\dot{\gamma}_{n-1}} \frac{n-1}{8}\left[n-3+(n-1) \frac{2 m}{r^{n-2}}\right] \\
& \times\left\{\frac{r^{p-2}}{r^{2}} \frac{\partial \psi^{2}}{\partial v^{*}}+\frac{r^{p-2}}{r^{2}} \sum_{i=1}^{\frac{n(n-1)}{2}} \frac{\partial\left(\Omega_{i} \psi\right)^{2}}{\partial v^{*}}+\frac{r^{p}}{r^{2}} \frac{\partial \chi^{2}}{\partial v^{*}}\right\} \\
& +\left.C(n, m, \delta, p, R) \int_{2 \tau_{1}+R^{*}}^{2 \tau_{2}+R^{*}} \mathrm{~d} t \int_{\mathbb{S}^{n-1}} \mathrm{~d} \mu_{\gamma_{n-1}}\left\{\sum_{i=1}^{\frac{n(n-1)}{2}}\left|\not \nabla \Omega_{i} \phi\right|^{2}+|\not \nabla \phi|^{2}+\left(\frac{\partial \psi}{\partial v^{*}}\right)^{2}+\psi^{2}\right\}\right|_{r=R},
\end{aligned}
$$


which, upon integration by parts, yields (5-40); note that the $\partial_{v^{*}} \psi^{2}$ and $\partial_{v^{*}}\left(\Omega_{i} \psi\right)^{2}$ terms generate boundary terms at infinity and zeroth-order bulk terms with the right sign by (5-16), while the $\partial_{v^{*}} \chi^{2}$ is reduced to a $\left(\partial_{v^{*}} \psi\right)^{2}$ term by (5-44).

By virtue of Stokes' theorem (B-5) and in view of (B-6), we conclude that

$$
\begin{aligned}
& \left.\int_{\tau_{2}+R^{*}}^{\infty} \mathrm{d} v^{*} \int_{\mathbb{S}^{n-1}} \mathrm{~d} \mu_{\dot{\gamma}_{n-1}}\left\{r^{p}\left(\frac{\partial \chi}{\partial v^{*}}\right)^{2}+r^{p-2}\left(\frac{\partial \psi}{\partial v^{*}}\right)^{2}+\sum_{i=1}^{\frac{n(n-1)}{2}} r^{p-2}\left(\frac{\partial \Omega_{i} \psi}{\partial v^{*}}\right)^{2}\right\}\right|_{u^{*}=\tau_{2}} \\
& +\int_{\tau_{1}}^{\tau_{2}} \mathrm{~d} u^{*} \int_{u^{*}+R^{*}}^{\infty} \mathrm{d} v^{*} \int_{\mathbb{S}^{n-1}} \mathrm{~d} \mu_{\dot{\gamma}_{n-1}} r^{p-1}\left(\frac{\partial \chi}{\partial v^{*}}\right)^{2} \\
& \leq C(n, m, \delta, p, R) \int_{\tau_{1}+R^{*}}^{\infty} \mathrm{d} v^{*} \int_{\mathbb{S}^{n-1}} \mathrm{~d} \mu_{\hat{\gamma}_{n-1}} \\
& \times\left.\left\{r^{p}\left(\frac{\partial \chi}{\partial v^{*}}\right)^{2}+r^{p-2}\left(\frac{\partial \psi}{\partial v^{*}}\right)^{2}+\sum_{i=1}^{\frac{n(n-1)}{2}} r^{p-2}\left(\frac{\partial \Omega_{i} \psi}{\partial v^{*}}\right)^{2}\right\}\right|_{u^{*}=\tau_{1}} \\
& +C(n, m, \delta, p, R) \int_{2 \tau_{1}+R^{*}}^{2 \tau_{2}+R^{*}} \mathrm{~d} t \int_{\mathbb{S}^{n-1}} \mathrm{~d} \mu_{\hat{\gamma}_{n-1}}\left\{\psi^{2}+\left(\frac{\partial \psi}{\partial v^{*}}\right)^{2}+\left(\frac{\partial^{2} \psi}{\partial v^{* 2}}\right)^{2}\right. \\
& +\sum_{i=1}^{\frac{n(n-1)}{2}}\left[\left(\Omega_{i} \psi\right)^{2}+\left(\frac{\partial \Omega_{i} \psi}{\partial v^{*}}\right)^{2}\right]+|\not \nabla|^{2}+(\not \nabla \psi)^{2} \\
& \left.+\sum_{i=1}^{\frac{n(n-1)}{2}}\left|\not \nabla \Omega_{i} \psi\right|^{2}\right\}\left.\right|_{r=R}
\end{aligned}
$$

Proof of Proposition 5.6. We shall use this weighted energy inequality for $\chi$ to proceed in a hierarchy of four steps.

$p=4-\delta:$ Let $\tau_{1}>0$ and $\tau_{j+1}=2 \tau_{j}(j \in \mathbb{N})$. In a first step we use (5-49) with $p=4-\delta$ and (5-17) with $p=2$ as an estimate for the spacetime integral of $\partial_{v^{*}} \chi, \partial_{v^{*}} \psi$, and $\partial_{v^{*}}\left(\Omega_{j} \psi\right)$ on ${ }^{R} \mathcal{D}_{\tau_{j}}^{\tau_{j+1}}$, and in a second step as an estimate for the corresponding integral on the future boundary of ${ }^{R} \mathcal{D}_{\tau_{1}}^{\tau_{j}}$ :

$$
\begin{aligned}
& \int_{\tau_{j}}^{\tau_{j+1}} \mathrm{~d} u^{*} \int_{u^{*}+R^{*}}^{\infty} \mathrm{d} v^{*} \int_{\mathbb{S}^{n-1}} \mathrm{~d} \mu_{\dot{\gamma}_{n-1}}\left\{r^{3-\delta}\left(\frac{\partial \chi}{\partial v^{*}}\right)^{2}\right.\left.+r\left(\frac{\partial \psi}{\partial v^{*}}\right)^{2}+\sum_{i=1}^{\frac{n(n-1)}{2}} r\left(\frac{\partial \Omega_{i} \psi}{\partial v^{*}}\right)^{2}\right\} \\
& \leq\left.C(n, m, \delta, R) \int_{\tau_{j}+R^{*}}^{\infty} \mathrm{d} v^{*} \int_{\mathbb{S}^{n-1}} \mathrm{~d} \mu_{\dot{\gamma}_{n-1}}\left\{r^{4-\delta}\left(\frac{\partial \chi}{\partial v^{*}}\right)^{2}+r^{2}\left(\frac{\partial \psi}{\partial v^{*}}\right)^{2}+\sum_{i=1}^{\frac{n(n-1)}{2}} r^{2}\left(\frac{\partial \Omega_{j} \psi}{\partial v^{*}}\right)^{2}\right\}\right|_{u^{*}=\tau_{j}} \\
&+C(n, m, \delta, R) \int_{2 \tau_{j}+R^{*}}^{2 \tau_{j+1}+R^{*}} \mathrm{~d} t \int_{\mathbb{S}^{n-1}} \mathrm{~d} \mu_{\gamma_{n-1}}\left\{\begin{array}{c}
\psi^{2}+\left(\frac{\partial \psi}{\partial v^{*}}\right)^{2}+\left(\frac{\partial^{2} \psi}{\partial v^{* 2}}\right)^{2}+|\not \nabla \psi|^{2}+\left|\not \nabla \frac{\partial \psi}{\partial v^{*}}\right|^{2} \\
+ \\
\left.+\sum_{i=1}^{\frac{n(n-1)}{2}}\left[\left(\Omega_{i} \psi\right)^{2}+\left(\frac{\partial \Omega_{i} \psi}{\partial v^{*}}\right)^{2}+\left|\not \nabla \Omega_{i} \psi\right|^{2}\right]\right\}\left.\right|_{r=R}
\end{array}\right.
\end{aligned}
$$




$$
\begin{aligned}
\leq & \left.C(n, m, \delta, R) \int_{\tau_{1}+R^{*}}^{\infty} \mathrm{d} v^{*} \int_{\mathbb{S}^{n-1}} \mathrm{~d} \mu_{\dot{\gamma}_{n-1}}\left\{r^{4-\delta}\left(\frac{\partial \chi}{\partial v^{*}}\right)^{2}+r^{2}\left(\frac{\partial \psi}{\partial v^{*}}\right)^{2}+\sum_{i=1}^{\frac{n(n-1)}{2}} r^{2}\left(\frac{\partial \Omega_{i} \psi}{\partial v^{*}}\right)^{2}\right\}\right|_{u^{*}=\tau_{1}} \\
+ & C(n, m, \delta, R) \int_{2 \tau_{1}+R^{*}}^{2 \tau_{j+1}+R^{*}} \mathrm{~d} t \int_{\mathbb{S}^{n-1}} \mathrm{~d} \mu_{\gamma_{n-1}}\left\{\psi^{2}+\left(\frac{\partial \psi}{\partial v^{*}}\right)^{2}+\left(\frac{\partial^{2} \psi}{\partial v^{* 2}}\right)^{2}+(\not \nabla)^{2}+\left|\not \nabla \frac{\partial \psi}{\partial v^{*}}\right|^{2}\right. \\
& \left.+\sum_{i=1}^{\frac{n(n-1)}{2}}\left[\left(\Omega_{i} \psi\right)^{2}+\left(\frac{\partial \Omega_{i} \psi}{\partial v^{*}}\right)^{2}+\left|\not \Omega_{i} \psi\right|^{2}\right]\right\}\left.\right|_{r=R}
\end{aligned}
$$

Thus by the mean value theorem of integration we obtain a sequence $\tau_{j}^{\prime} \in\left(\tau_{j}, \tau_{j+1}\right)(j \in \mathbb{N})$ such that the corresponding integral from the left-hand side on $u^{*}=\tau_{j}^{\prime}$ is bounded by $\tau_{j}^{-1}$ times the right-hand side of (5-50).

$p=3-\delta$ : Next we shall use (5-49) with $p=3-\delta$ on $R_{j} \mathcal{D}_{\tau_{2 j-1}^{\prime}}^{\tau_{2 j+1}^{\prime}}$ (with $R_{j}^{*} \in\left(R^{*}, R^{*}+1\right)(j \in \mathbb{N})$ chosen appropriately below). However, the quantity we are actually interested in is not $\partial_{v^{*}} \chi$, but rather

$$
\begin{aligned}
\left(\frac{\partial r^{\frac{n-1}{2}} T \cdot \phi}{\partial v^{*}}\right)^{2} & =\left(\frac{\partial T \cdot r^{\frac{n-1}{2}} \phi}{\partial v^{*}}\right)^{2}=\left(\frac{1}{2} \frac{\partial^{2} \psi}{\partial v^{* 2}}+\frac{1}{2} \frac{\partial^{2} \psi}{\partial u^{*} \partial v^{*}}\right)^{2} \\
& \stackrel{(5-12)}{=}\left(\frac{1}{2} \frac{\partial^{2} \psi}{\partial v^{* 2}}+\frac{1}{2}\left(1-\frac{2 m}{r^{n-2}}\right) \phi \psi-\frac{1}{2} \frac{n-1}{2}\left(\frac{n-3}{2}+\frac{n-1}{2} \frac{2 m}{r^{n-2}}\right) \frac{1}{r^{2}}\left(1-\frac{2 m}{r^{n-2}}\right) \psi\right)^{2} \\
& \leq\left(\frac{\partial^{2} \psi}{\partial v^{* 2}}\right)^{2}+(\Delta \psi)^{2}+\frac{n-1}{4} 2(n-2) \frac{1}{r^{4}} \psi^{2}
\end{aligned}
$$

Using the simple Hardy inequality

$\frac{1}{2} \int_{u^{*}+R^{*}}^{\infty} \mathrm{d} v^{*} r^{2-\delta} \frac{1}{r^{4}} \psi^{2}$

$$
\leq \frac{1}{1-\frac{2 m}{R^{n-2}}} \frac{1}{r^{1+\delta}} \psi^{2}\left(u^{*}, u^{*}+R^{*}\right)+\frac{2}{\left(1-\frac{2 m}{R^{n-2}}\right)^{2}} \int_{u^{*}+R^{*}}^{\infty} \mathrm{d} v^{*} \frac{1}{r^{\delta}}\left(\frac{\partial \psi}{\partial v^{*}}\right)^{2}
$$

and again the commutation introduced in Lemma 5.7, we obtain

$$
\begin{aligned}
& \int_{\tau_{2 j-1}^{\prime}}^{\tau_{2 j+1}^{\prime}} \mathrm{d} u^{*} \int_{u^{*}+R_{j}}^{\infty} \mathrm{d} v^{*} \int_{\mathbb{S}^{n-1}} \mathrm{~d} \mu_{\dot{\gamma}_{n-1}} r^{2-\delta}\left(\frac{\partial r^{\frac{n-1}{2}} T \cdot \phi}{\partial v^{*}}\right)^{2} \\
& \leq \int_{\tau_{2 j-1}^{\prime}}^{\tau_{2 j+1}^{\prime}} \mathrm{d} u^{*} \int_{u^{*}+R_{j}}^{\infty} \mathrm{d} v^{*} \int_{\mathbb{S}^{n-1}} \mathrm{~d} \mu_{\dot{\gamma}_{n-1}}\left\{r^{2-\delta}\left(\frac{\partial \chi}{\partial v^{*}}\right)^{2}\right. \\
& \left.+\frac{C}{r^{\delta}} \sum_{i=1}^{\frac{n(n-1)}{2}}\left|\not \nabla \Omega_{i} \psi\right|^{2}+\frac{C}{r^{\delta}}(\not \nabla)^{2}+\frac{(n-1)(n-2)}{2} \frac{2}{\left(1-\frac{2 m}{R^{n-2}}\right)^{2}} r^{-\delta}\left(\frac{\partial \psi}{\partial v^{*}}\right)^{2}\right\} \\
& +\left.\frac{1}{1-\frac{2 m}{R^{n-2}}} \int_{2 \tau_{2 j-1}^{\prime}+R_{j}^{*}}^{2 \tau_{2 j+1}^{\prime}+R_{j}^{*}} \mathrm{~d} t \int_{\mathbb{S}^{n-1}} \mathrm{~d} \mu_{\dot{\gamma}_{n-1}}\left\{\frac{1}{r^{1+\delta}} \psi^{2}\right\}\right|_{r=R_{j}}
\end{aligned}
$$




$$
\begin{aligned}
& \leq C(n, m, \delta) \int_{\tau_{2 j-1}^{\prime}}^{\tau_{2 j+1}^{\prime}} \mathrm{d} u^{*} \int_{u^{*}+R_{j}^{*}}^{\infty} \mathrm{d} v^{*} \int_{\mathbb{S}^{n-1}} \mathrm{~d} \mu_{\dot{\gamma}_{n-1}}\left\{r^{2-\delta}\left(\frac{\partial \chi}{\partial v^{*}}\right)^{2}\right. \\
& \left.+\stackrel{r}{K}_{1-\delta}(\phi) r^{n-1}+\sum_{i=1}^{\frac{n(n-1)}{2}} \stackrel{r}{K}_{1-\delta}\left(\Omega_{i} \phi\right) r^{n-1}\right\} \\
& +\left.C(n, m, \delta) \int_{2 \tau_{2 j-1}^{\prime}+R_{j}^{*}}^{2 \tau_{2 j+1}^{\prime}+R_{j}^{*}} \mathrm{~d} t \int_{\mathbb{S}^{n-1}} \mathrm{~d} \mu_{\dot{\gamma}_{n-1}}\left\{\psi^{2}+\sum_{i=1}^{\frac{n(n-1)}{2}}\left(\Omega_{i} \psi\right)^{2}\right\}\right|_{r=R_{j}},
\end{aligned}
$$

where in the last step we have again used (5-16). Furthermore, by now applying (5-49) with $p=3-\delta$,

$$
\begin{aligned}
& \int_{\tau_{2 j-1}^{\prime}}^{\tau_{2 j+1}^{\prime}} \mathrm{d} u^{*} \int_{u^{*}+R_{j}}^{\infty} \mathrm{d} v^{*} \int_{\mathbb{S}^{n-1}} \mathrm{~d} \mu_{\dot{\gamma}_{n-1}} r^{2-\delta}\left(\frac{\partial r^{\frac{n-1}{2}} T \cdot \phi}{\partial v^{*}}\right)^{2} \\
& \leq\left. C(n, m, \delta, R) \int_{\tau_{2 j-1}^{\prime}+R_{j}^{*}}^{\infty} \mathrm{d} v^{*} \int_{\mathbb{S}^{n-1}} \mathrm{~d} \mu_{\dot{\gamma}_{n-1}}\left\{r^{3-\delta}\left(\frac{\partial \chi}{\partial v^{*}}\right)^{2}+r\left(\frac{\partial \psi}{\partial v^{*}}\right)^{2}+\sum_{i=1}^{\frac{n(n-1)}{2}} r\left(\frac{\partial \Omega_{i} \psi}{\partial v^{*}}\right)^{2}\right\}\right|_{u^{*}=\tau_{2 j-1}^{\prime}} \\
& \quad+C(n, m, \delta, R) \int_{2 \tau_{2 j-1}^{\prime}+R_{j}^{*}}^{2 \tau_{2 j+1}^{\prime}+R_{j}^{*}} \mathrm{~d} t \int_{\mathbb{S}^{n-1}} \mathrm{~d} \mu_{\dot{\gamma}_{n-1}}\left\{\psi^{2}+\left(\frac{\partial \psi}{\partial v^{*}}\right)^{2}+\left(\frac{\partial^{2} \psi}{\partial v^{* 2}}\right)^{2}+(\not \nabla)^{2}+\left|\nabla \frac{\partial \psi}{\partial v^{*}}\right|^{2}\right. \\
& \left.+\sum_{i=1}^{\frac{n(n-1)}{2}}\left[\left(\Omega_{i} \psi\right)^{2}+\left(\frac{\partial \Omega_{i} \psi}{\partial v^{*}}\right)^{2}+\left|\nabla \Omega_{i} \psi\right|^{2}\right]\right\}\left.\right|_{r=R_{j}}, \quad(5-54)
\end{aligned}
$$

we obtain a sequence $\tau_{j}^{\prime \prime} \in\left(\tau_{2 j-1}^{\prime}, \tau_{2 j+1}^{\prime}\right)(j \in \mathbb{N})$ such that, in view of the previous step,

$$
\begin{aligned}
& \left.\int_{\tau_{j}^{\prime \prime}+R_{j}^{*}}^{\infty} \mathrm{d} v^{*} \int_{\mathbb{S}^{n-1}} \mathrm{~d} \mu_{\dot{\gamma}_{n-1}}\left\{r^{2-\delta}\left(\frac{\partial\left(r^{\frac{n-1}{2}} T \cdot \phi\right)}{\partial v^{*}}\right)^{2}\right\}\right|_{u^{*}=\tau_{j}^{\prime \prime}} \\
& \leq\left.\frac{C(n, m, \delta, R)}{\tau_{2 j} \tau_{2 j-1}} \int_{\tau_{1}+R^{*}}^{\infty} \mathrm{d} v^{*} \int_{\mathbb{S}^{n-1}} \mathrm{~d} \mu_{\gamma_{n-1}}\left\{r^{4-\delta}\left(\frac{\partial \chi}{\partial v^{*}}\right)^{2}+r^{2}\left(\frac{\partial \psi}{\partial v^{*}}\right)^{2}+r^{2} \sum_{i=1}^{\frac{n(n-1)}{2}}\left(\frac{\partial \Omega_{i} \psi}{\partial v^{*}}\right)^{2}\right\}\right|_{u^{*}=\tau_{1}} \\
& +\frac{C(n, m, \delta, R)}{\tau_{2 j} \tau_{2 j-1}} \int_{2 \tau_{1}+R^{*}}^{2 \tau_{2 j+1}+R^{*}} \mathrm{~d} t \int_{\mathbb{S}^{n-1}} \mathrm{~d} \mu_{\dot{\gamma}_{n-1}}\left\{\psi^{2}+\left(\frac{\partial \psi}{\partial v^{*}}\right)^{2}+\left(\frac{\partial^{2} \psi}{\partial v^{* 2}}\right)^{2}+(\not \nabla)^{2}+\left|\nabla \frac{\partial \psi}{\partial v^{*}}\right|^{2}\right. \\
& \left.+\sum_{i=1}^{\frac{n(n-1)}{2}}\left[\left(\Omega_{i} \psi\right)^{2}+\left(\frac{\partial \Omega_{i} \psi}{\partial v^{*}}\right)^{2}+\left|\not \nabla \Omega_{i} \psi\right|^{2}\right]\right\}\left.\right|_{r=R} \\
& +C(n, m, \delta, R) \int_{2 \tau_{2 j-1}^{\prime}+R_{j}^{*}}^{2 \tau_{2 j+1}^{\prime}+R_{j}^{*}} \mathrm{~d} t \int_{\mathbb{S}^{n-1}} \mathrm{~d} \mu_{\dot{\gamma}_{n-1}}\left\{\psi^{2}+\left(\frac{\partial \psi}{\partial v^{*}}\right)^{2}+\left(\frac{\partial^{2} \psi}{\partial v^{* 2}}\right)^{2}+(\not \nabla \psi)^{2}+\left|\not \nabla \frac{\partial \psi}{\partial v^{*}}\right|^{2}\right. \\
& \left.+\sum_{i=1}^{\frac{n(n-1)}{2}}\left[\left(\Omega_{i} \psi\right)^{2}+\left(\frac{\partial \Omega_{i} \psi}{\partial v^{*}}\right)^{2}+\left|\not \Omega_{i} \psi\right|^{2}\right]\right\}\left.\right|_{r=R_{j}} .
\end{aligned}
$$


Now, by writing out the derivatives of $\psi=r^{\frac{n-1}{2}} \phi$, and using (5-12), we calculate that

$$
\begin{array}{r}
\left.\int_{\mathbb{S}^{n-1}} \mathrm{~d} \mu_{\dot{\gamma}_{n-1}}\left\{\psi^{2}+\left(\frac{\partial \psi}{\partial v^{*}}\right)^{2}+\left(\frac{\partial^{2} \psi}{\partial v^{* 2}}\right)^{2}+|\not \nabla|^{2}+\left|\not \frac{\partial \psi}{\partial v^{*}}\right|^{2}+\sum_{i=1}^{\frac{n(n-1)}{2}}\left[\left(\Omega_{i} \psi\right)^{2}+\left(\frac{\partial \Omega_{i} \psi}{\partial v^{*}}\right)^{2}+\left|\not \Omega_{i} \psi\right|^{2}\right]\right\}\right|_{r=R} \\
\leq C(R) \int_{\mathbb{S}^{n-1}} \mathrm{~d} \mu_{\dot{\gamma}_{n-1}}\left\{\phi^{2}+\left(\frac{\partial \phi}{\partial v^{*}}\right)^{2}+\left(\frac{\partial \phi}{\partial u^{*}}\right)^{2}+\left(\frac{\partial T \cdot \phi}{\partial v^{*}}\right)^{2}+|\not \nabla|^{2}\right. \\
\left.+\sum_{i=1}^{\frac{n(n-1)}{2}}\left[\left|\not \nabla \Omega_{i} \phi\right|^{2}+\left(\frac{\partial \Omega_{i} \phi}{\partial v^{*}}\right)^{2}\right]\right\}\left.\right|_{r=R} \quad \text { (5-56) }
\end{array}
$$

by applying Proposition 4.1 first to the domain ${ }^{r_{1}} \mathcal{D}_{\tau_{1}}^{\tau_{2 j+1}} \subset \mathcal{R}_{r_{0}, r_{1}}^{\infty}\left(2 \tau_{1}+r_{1}^{*}\right)$ where $r_{1}>\sqrt[n-2]{\frac{4 n m}{\delta}}$ to fix the radius $R$, and then to the domain

$$
r\left(r^{*}=R^{*}+1\right) \mathcal{D}_{\tau_{2 j-1}^{\prime}}^{\tau_{2 j+1}^{\prime} \backslash R} \mathcal{D}_{\tau_{2 j-1}^{\prime}}^{\tau_{2 j+1}^{\prime}} \subset \mathcal{R}_{r_{0}, R}^{\infty}\left(2 \tau_{2 j-1}^{\prime}+R^{*}\right)
$$

to fix the radii $R_{j}(j \in \mathbb{N})$ by using the mean value theorem for the integration in $r^{*}$, this yields (see also Appendix B)

$$
\begin{aligned}
& \left.\int_{\tau_{j}^{\prime \prime}+R_{j}^{*}}^{\infty} \mathrm{d} v^{*} \int_{\mathbb{S}^{n-1}} \mathrm{~d} \mu_{\dot{\gamma}_{n-1}}\left\{r^{2-\delta}\left(\frac{\partial r^{\frac{n-1}{2}} T \cdot \phi}{\partial v^{*}}\right)^{2}\right\}\right|_{u^{*}=\tau_{j}^{\prime \prime}} \\
& \leq \frac{C(n, m, \delta, R)}{\left(\tau_{j}^{\prime \prime}\right)^{2}}\left\{\left.\int_{\tau_{1}+R^{*}}^{\infty} \mathrm{d} v^{*} \int_{\mathbb{S}^{n-1}} \mathrm{~d} \mu_{\gamma_{n-1}}\left\{r^{4-\delta}\left(\frac{\partial \chi}{\partial v^{*}}\right)^{2}+r^{2}\left(\frac{\partial \psi}{\partial v^{*}}\right)^{2}+r^{2} \sum_{i=1}^{\frac{n(n-1)}{2}}\left(\frac{\partial \Omega_{i} \psi}{\partial v^{*}}\right)^{2}\right\}\right|_{u^{*}=\tau_{1}}\right. \\
& \left.+\int_{\Sigma_{\tau_{1}}}\left(J^{T}(\phi)+J^{T}(T \cdot \phi)+J^{T}\left(T^{2} \cdot \phi\right)+\sum_{i=1}^{2}\left[J^{T}\left(\Omega_{i} \phi\right)+J^{T}\left(T \cdot \Omega_{i} \phi\right)\right], n\right)\right\} \\
& +C(n, m, \delta, R) \int_{\Sigma_{\tau_{2 j}-1}}\left(J^{T}(\phi)+J^{T}(T \cdot \phi)+J^{T}\left(T^{2} \cdot \phi\right)+\sum_{i=1}^{2}\left[J^{T}\left(\Omega_{i} \phi\right)+J^{T}\left(T \cdot \Omega_{i} \phi\right)\right], n\right) .(5-57)
\end{aligned}
$$

Therefore, by Proposition 5.4,

$$
\begin{gathered}
\left.\int_{\tau_{j}^{\prime \prime}+R_{j}^{*}}^{\infty} \mathrm{d} v^{*} \int_{\mathbb{S}^{n-1}} \mathrm{~d} \mu_{\dot{\gamma}_{n-1}}\left\{r^{2-\delta}\left(\frac{\partial r^{\frac{n-1}{2}} T \cdot \phi}{\partial v^{*}}\right)^{2}\right\}\right|_{u^{*}=\tau_{j}^{\prime \prime}} \\
\leq \frac{C(n, m, \delta, R)}{\left(\tau_{j}^{\prime \prime}\right)^{2}}\left\{\left.\int_{\tau_{1}+R^{*}}^{\infty} \mathrm{d} v^{*} \int_{\mathbb{S}^{n-1}} \mathrm{~d} \mu_{\dot{\gamma}_{n-1}}\left\{r^{4-\delta}\left(\frac{\partial \chi}{\partial v^{*}}\right)^{2}+\sum_{k=0}^{3} r^{2}\left(\frac{\partial T^{k} \cdot \psi}{\partial v^{*}}\right)^{2}+\sum_{k=0}^{2} \frac{n(n-1)}{\sum_{i=1}^{2}} r^{2}\left(\frac{\partial T^{k} \Omega_{i} \psi}{\partial v^{*}}\right)^{2}\right\}\right|_{u^{*}=\tau_{1}}\right. \\
\left.+\int_{\Sigma_{\tau_{1}}}\left(\sum_{k=0}^{4} J^{N}\left(T^{k} \cdot \phi\right)+\sum_{k=0}^{3} \sum_{i=1}^{\frac{n(n-1)}{2}} J^{N}\left(T^{k} \Omega_{i} \phi\right), n\right)\right\}
\end{gathered}
$$


Remark 5.8. This statement should be compared to the assumptions of Proposition 5.4 (5-5), from which all that one can deduce with (5-17) is

$$
\left.\int_{\tau+R^{*}}^{\infty} \mathrm{d} v^{*} \int_{\mathbb{S}^{n-1}} \mathrm{~d} \mu_{\gamma_{n-1}}\left\{r^{2}\left(\frac{\partial\left(r^{\frac{n-1}{2}} T \cdot \phi\right)}{\partial v^{*}}\right)^{2}\right\}\right|_{u^{*}=\tau}<\infty \quad\left(\tau>\tau_{0}\right) .
$$

We shall now proceed along the lines of the proof of Proposition 5.4 in Section 5B, just that we have (5-58) as a starting point for the solution $T \cdot \phi$ of (1-1) (and (5-6)); however, as opposed to Proposition 5.4 the hierarchy does not descend from $p=2$ but $p<2$, which introduces a degeneracy in the last step, and requires the refinement of Proposition 4.1 to Proposition 4.21, and Proposition 4.4 to Proposition 4.22; see Section 4D.2.

Lemma 5.9 (pointwise decay under special assumptions). Let $\phi$ be a solution of the wave equation (1-1), with initial data on $\Sigma_{\tau_{1}}\left(\tau_{1}>0\right)$ satisfying

$$
\begin{aligned}
D \doteq \int_{\tau_{1}+R^{*}}^{\infty} \mathrm{d} v^{*} \int_{\mathbb{S}^{n-1}} \mathrm{~d} \mu_{\dot{\gamma}_{n-1}}\left\{r^{4-\delta}\left(\frac{\partial \chi}{\partial v^{*}}\right)^{2}\right. & \left.+\sum_{k=0}^{3} r^{2}\left(\frac{\partial T^{k} \psi}{\partial v^{*}}\right)^{2}+\sum_{k=0}^{2} \frac{\sum_{i=1}^{\frac{n(n-1)}{2}}}{2} r^{2}\left(\frac{\partial T^{k} \Omega_{i} \psi}{\partial v^{*}}\right)^{2}\right\}\left.\right|_{u^{*}=\tau_{1}} \\
& +\int_{\Sigma_{\tau_{1}}}\left(\sum_{k=0}^{4} J^{N}\left(T^{k} \cdot \phi\right)+\sum_{k=0}^{3} \sum_{i=1}^{\frac{n(n-1)}{2}} J^{N}\left(T^{k} \Omega_{i} \phi\right), n\right)<\infty
\end{aligned}
$$

for some $\delta>0$ and

$$
\int_{\tau^{\prime}+R^{*}}^{\infty} \mathrm{d} v^{*} \int_{\mathbb{S}^{n-1}} \mathrm{~d} \mu_{\dot{\gamma}_{n-1}} \times\left. r^{2-\delta}\left(\frac{\partial T \cdot \psi}{\partial v^{*}}\right)^{2}\right|_{u^{*}=\tau^{\prime}} \leq \frac{C(n, m, \delta, R) D}{\tau^{\prime 2}}
$$

for some $\tau^{\prime}>\tau_{1}$. Then there is a constant $C(n, m, \delta, R)$ such that, for all $\tau>\tau^{\prime}$,

$$
\left.\int_{\mathbb{S}^{n-1}} \mathrm{~d} \mu_{\dot{\gamma}_{n-1}} r^{n-1-\frac{\delta}{2}}(T \cdot \phi)^{2}\right|_{\left(u^{*}=\tau^{\prime}, v^{*}=R^{*}+\tau\right)} \leq \frac{C D}{\tau^{\prime 2}} .
$$

Remark 5.10. Note the gain in powers of $r$ in comparison to the boundary term arising in Proposition 4.22. Proof. First, integrating from infinity,

$$
(T \cdot \phi)\left(\tau^{\prime}, R^{*}+\tau^{\prime}\right)=-\int_{\tau^{\prime}+R^{*}}^{\infty} \frac{\partial(T \cdot \phi)}{\partial v^{*}} \mathrm{~d} v^{*},
$$

and then, by Cauchy's inequality,

$$
\begin{aligned}
& \int_{\mathbb{S}^{n-1}} \mathrm{~d} \mu_{\dot{\gamma}_{n-1}}(T \cdot \phi)^{2}\left(\tau^{\prime}, R+\tau^{\prime}\right) \\
& \quad \leq \int_{R^{*}+\tau^{\prime}}^{\infty} \frac{1}{r^{n-1}} \mathrm{~d} v^{*} \times \int_{R+\tau^{\prime}}^{\infty} \int_{\mathbb{S}^{n-1}} \mathrm{~d} \mu_{\dot{\gamma}_{n-1}}\left(\frac{\partial(T \cdot \phi)}{\partial v^{*}}\right)^{2} r^{n-1} \mathrm{~d} v^{*} \\
& \quad \leq \frac{1}{2}\left(1-\frac{2 m}{\left.r^{n-2}\right|_{\left(u^{*}=\tau^{\prime}, v^{*}=R^{*}+\tau^{\prime}\right)}}\right)^{-1} \frac{1}{n-2} \frac{1}{r^{n-2}} C(m, n) \int_{R^{*}+\tau^{\prime}}^{\infty} \mathrm{d} v^{*} \int_{\mathbb{S}^{n-1}} \mathrm{~d} \mu_{\dot{\gamma}_{n-1}} r^{n-1}\left(\frac{\partial(T \cdot \phi)}{\partial v^{*}}\right)^{2} .
\end{aligned}
$$


Therefore, by Proposition 5.4,

$$
\int_{\mathbb{S}^{n-1}} \mathrm{~d} \mu_{\dot{\gamma}_{n-1}}\left(r^{n-2}(T \cdot \phi)^{2}\right)\left(\tau^{\prime}, R^{*}+\tau^{\prime}\right) \leq \frac{C(n, m)}{1-\frac{2 m}{R^{n-2}}} \int_{\Sigma_{\tau^{\prime}}}\left(J^{T}(T \cdot \phi), n\right) \leq \frac{C(n, m, R)}{\tau^{\prime 2}} D .
$$

Now

$$
\begin{aligned}
& r^{n-1} \int_{\mathbb{S}^{n-1}} \mathrm{~d} \mu_{\dot{\gamma}_{n-1}}(T \cdot \phi)^{2}\left(\tau^{\prime}, R^{*}+\tau\right) \\
& =\int_{\mathbb{S}^{n-1}} \mathrm{~d} \mu_{\dot{\gamma}_{n-1}}\left(r^{n-1}(T \cdot \phi)^{2}\right)\left(\tau^{\prime}, R^{*}+\tau^{\prime}\right)+\int_{R^{*}+\tau^{\prime}}^{R^{*}+\tau} \mathrm{d} v^{*} \int_{\mathbb{S}^{n-1}} \mathrm{~d} \mu_{\dot{\gamma}_{n-1}} 2 T \cdot \psi \frac{\partial T \cdot \psi}{\partial v^{*}} \\
& \leq R^{n-1} \int_{\mathbb{S}^{n-1}} \mathrm{~d} \mu_{\dot{\gamma}_{n-1}}(T \cdot \phi)^{2}\left(\tau^{\prime}, R^{*}+\tau^{\prime}\right) \\
& \quad+\left.2 r^{\frac{\delta}{2}}\right|_{\substack{\left(u^{*}=\tau^{\prime}, v^{*}=R^{*}+\tau\right)}} \sqrt{\int_{R^{*}+\tau^{\prime}}^{\infty} \mathrm{d} v^{*} \int_{\mathbb{S}^{n-1}} \mathrm{~d} \mu_{\dot{\gamma}_{n-1}} \frac{1}{r^{2}}(T \cdot \phi)^{2} r^{n-1}} \sqrt{\int_{R^{*}+\tau^{\prime}}^{\infty} \mathrm{d} v^{*} \int_{\mathbb{S}^{n-1}} \mathrm{~d} \mu_{\dot{\gamma}_{n-1}} r^{2-\delta}\left(\frac{\partial T \cdot \psi}{\partial v^{*}}\right)^{2}},
\end{aligned}
$$

which proves the pointwise estimate of the lemma in view of the Hardy inequality of Lemma C.2, Proposition 5.4, the assumption $(*)$ and $(* *)$.

$p=2-\delta:$ By the weighted energy inequality with $p=2-\delta$ and $r^{\frac{n-1}{2}} T \cdot \phi$ in the role of $\psi$ (see (5-16) in particular),

$$
\begin{aligned}
& \int_{\tau_{2 j-1}^{\prime \prime}}^{\tau_{2 j+1}^{\prime \prime}} \mathrm{d} u^{*} \int_{u^{*}+R_{j}^{\prime}}^{\infty} \mathrm{d} v^{*} \int_{\mathbb{S}^{n-1}} \mathrm{~d} \mu_{\dot{\gamma}_{n-1}} r^{1-\delta}\left(\frac{\partial T \cdot \psi}{\partial v^{*}}\right)^{2} \\
& \leq C(n, m) \int_{\tau_{2 j-1}^{\prime \prime}}^{\tau_{2 j+1}^{\prime \prime}} \mathrm{d} u^{*} \int_{u^{*}+R_{j}^{\prime}}^{\infty} \mathrm{d} v^{*} \int_{\mathbb{S}^{n-1}} \mathrm{~d} \mu_{\dot{\gamma}_{n-1}} \stackrel{r}{K_{2-\delta}}(T \cdot \phi) \\
& +\left.C(n, m) \int_{2 \tau_{2 j-1}^{\prime \prime}+R_{j}^{\prime}}^{2 \tau_{2 j+1}^{\prime \prime}+R_{j}^{\prime *}} \mathrm{~d} t \int_{\mathbb{S} n-1} \mathrm{~d} \mu_{\dot{\gamma}_{n-1}}\left\{(T \cdot \psi)^{2}\right\}\right|_{r=R_{j}^{\prime}} \\
& \leq\left. C(n, m, R) \int_{\tau_{2 j-1}^{\prime \prime}+R_{j}^{\prime}}^{\infty} \mathrm{d} v^{*} \int_{\mathbb{S}^{n-1}} \mathrm{~d} \mu_{\dot{\gamma}_{n-1}}\left\{r^{2-\delta}\left(\frac{\partial T \cdot \psi}{\partial v^{*}}\right)^{2}\right\}\right|_{u^{*}=\tau_{2 j-1}^{\prime \prime}} \\
& +\left.C(n, m) \int_{2 \tau_{2 j-1}^{\prime \prime}+R_{j}^{\prime}}^{2 \tau_{2 j+1}^{\prime \prime}+R_{j}^{\prime *}} \mathrm{~d} t \int_{\mathbb{S}^{n-1}} \mathrm{~d} \mu_{\dot{\gamma}_{n-1}}\left\{(T \cdot \psi)^{2}+\left(\frac{\partial T \cdot \psi}{\partial v^{*}}\right)^{2}+|\not T \cdot \psi|^{2}\right\}\right|_{r=R_{j}^{\prime}},
\end{aligned}
$$

where we choose $R_{j}^{\prime *} \in\left(R^{*}+1, R^{*}+2\right)$ such that Proposition 4.1 applied to the domain

$$
r\left(r^{*}=R^{*}+2\right) \mathcal{D}_{\tau_{2 j-1}^{\prime \prime}}^{\tau_{2 j+1}^{\prime \prime}} \backslash r\left(r^{*}=R^{*}+1\right) \mathcal{D}_{\tau_{2 j-1}^{\prime \prime}}^{\tau_{2 j+1}^{\prime \prime}}
$$

yields an estimate for the integral on the timelike boundary above in terms of the first- and secondorder energies on $\Sigma_{\tau_{2 j-1}^{\prime \prime}}$, which in turn decays by Proposition 5.4. Therefore there exists a sequence 
$\tau_{j}^{\prime \prime \prime} \in\left(\tau_{2 j-1}^{\prime \prime}, \tau_{2 j+1}^{\prime \prime}\right)(j \in \mathbb{N})$ such that

$$
\begin{gathered}
\left.\int_{\tau_{j}^{\prime \prime \prime}+R_{j}^{\prime *}}^{\infty} \mathrm{d} v^{*} \int_{\mathbb{S}^{n-1}} \mathrm{~d} \mu_{\dot{\gamma}_{n-1}}\left\{r^{1-\delta}\left(\frac{\partial T \cdot \psi}{\partial v^{*}}\right)^{2}\right\}\right|_{u^{*}=\tau_{j}^{\prime \prime \prime}} \\
\leq \frac{C(n, m, \delta, R)}{\left(\tau_{j}^{\prime \prime \prime}\right)^{3}}\left\{\int _ { \tau _ { 1 } + R ^ { * } } ^ { \infty } \mathrm { d } v ^ { * } \int _ { \mathbb { S } ^ { n - 1 } } \mathrm { d } \mu _ { \dot { \gamma } _ { n - 1 } } \left\{r^{4-\delta}\left(\frac{\partial \chi}{\partial v^{*}}\right)^{2}+\sum_{k=0}^{3} r^{2}\left(\frac{\partial\left(T^{k} \cdot \psi\right)}{\partial v^{*}}\right)^{2}\right.\right. \\
+\left.\sum_{\Sigma_{\tau_{1}}}\left(\sum_{k=0}^{2} \sum_{i=1}^{\frac{n(n-1)}{2}} r^{2}\left(\frac{\partial T^{k} \Omega_{i} \psi}{\partial v^{*}}\right)^{2}\right\}\right|_{u^{*}=\tau_{1}} \\
\left.\left.\quad+T_{k=0}^{3} \frac{n(n-1)}{\sum_{i=1}^{2}} J^{N}\left(T^{k} \Omega_{i} \phi\right), n\right)\right\} .
\end{gathered}
$$

$p=1-\delta:$ Since, by integrating by parts,

$$
\begin{aligned}
& \int_{u^{*}+R^{*}}^{\infty} \mathrm{d} v^{*} \frac{1}{r^{\delta}}\left(\frac{\partial \psi}{\partial v^{*}}\right)^{2} \\
& =\int_{u^{*}+R^{*}}^{\infty} \mathrm{d} v^{*} \frac{1}{r^{\delta}}\left\{\frac{n-1}{2 r} r^{\frac{n-1}{2}}\left(1-\frac{2 m}{r^{n-2}}\right) \frac{\partial\left(r^{\frac{n-1}{2}} \phi^{2}\right)}{\partial v^{*}}+r^{n-1}\left(\frac{\partial \phi}{\partial v^{*}}\right)^{2}\right\} \\
& =\left.\frac{1}{r^{\delta}} \frac{n-1}{2 r}\left(1-\frac{2 m}{r^{n-2}}\right) \psi^{2}\right|_{u^{*}+R^{*}} ^{\infty} \\
& +\int_{u^{*}+R^{*}}^{\infty}\left\{\frac{\delta}{r^{1+\delta}} \frac{n-1}{2 r}\left(1-\frac{2 m}{r^{n-2}}\right)^{2} \psi^{2}\right. \\
& \left.\quad+\frac{1}{r^{\delta}} \frac{n-1}{2 r^{2}}\left(1-\frac{2 m}{r^{n-2}}\right) \psi^{2}\left[(n-2)+\left(1-\frac{2 m}{r^{n-2}}\right) \frac{n-3}{2}\right]+\frac{1}{r^{\delta}}\left(\frac{\partial \phi}{\partial v^{*}}\right)^{2} r^{n-1}\right\},
\end{aligned}
$$

we have by (5-15) that also (with $R_{j}^{\prime \prime *} \in\left(R^{*}+2, R^{*}+3\right)$ )

$$
\begin{aligned}
& \int_{\tau_{2 j-1}^{\prime \prime \prime}}^{\tau_{2 j+1}^{\prime \prime \prime}} \mathrm{d} u^{*} \int_{u^{*}+R_{j}^{\prime \prime *}}^{\infty} \mathrm{d} v^{*} \int_{\mathbb{S}^{n-1}} \mathrm{~d} \mu_{\dot{\gamma}_{n-1}} \frac{1}{r^{\delta}}\left\{\left(\frac{\partial T \cdot \phi}{\partial v^{*}}\right)^{2}+|\not \nabla T \cdot \phi|^{2}\right\} r^{n-1} \\
& \leq C(n, m)\left\{\int_{\tau_{2 j-1}^{\prime \prime \prime}}^{\tau_{2 \prime \prime \prime 1}^{\prime \prime \prime}} \mathrm{d} u^{*} \int_{u^{*}+R_{j}^{\prime \prime *}}^{\infty} \mathrm{d} v^{*} \int_{\mathbb{S}^{n-1}} \mathrm{~d} \mu_{\dot{\gamma}_{n-1}} \stackrel{r}{K}_{1-\delta}(T \cdot \phi) \cdot r^{n-1}\right. \\
& \left.+\left.\int_{2 \tau_{2 j-1}^{\prime \prime \prime}+R_{j}^{\prime \prime *}}^{2 \tau_{2 j+1}^{\prime \prime \prime}+R_{j}^{\prime \prime *}} \mathrm{~d} t \int_{\mathbb{S}^{n-1}} \mathrm{~d} \mu_{\hat{\gamma}_{n-1}}\left\{(T \cdot \psi)^{2}\right\}\right|_{r=R_{j}^{\prime \prime}}\right\} .
\end{aligned}
$$

By virtue of Stokes' theorem (B-5), (B-6) and our previous result (5-61), we obtain 


$$
\begin{aligned}
& \int_{\tau_{2 j-1}^{\prime \prime \prime}}^{\tau_{2 j+1}^{\prime \prime \prime}} \mathrm{d} u^{*} \int_{u^{*}+R_{j}^{\prime \prime}}^{\infty} \mathrm{d} v^{*} \int_{\mathbb{S}^{n-1}} \mathrm{~d} \mu_{\dot{\gamma}_{n-1}} \frac{1}{r^{\delta}}\left(J^{T}(T \cdot \phi), \frac{\partial}{\partial v^{*}}\right) r^{n-1} \\
& \leq C(n, m)\left\{\left.\int_{\tau_{2 j-1}^{\prime \prime \prime}+R_{j}^{\prime \prime}}^{\infty} \mathrm{d} v^{*} \int_{\mathbb{S}^{n-1}} \mathrm{~d} \mu_{\dot{\gamma}_{n-1}}\left\{r^{1-\delta}\left(\frac{\partial(T \cdot \psi)}{\partial v^{*}}\right)^{2}\right\}\right|_{u^{*}=\tau_{2 j-1}^{\prime \prime \prime}}\right. \\
& \left.+\left.\int_{2 \tau_{2 j-1}^{\prime \prime \prime}+R_{j}^{\prime *}}^{2 \tau_{2 j+1}^{\prime \prime \prime}+R_{j}^{\prime \prime *}} \mathrm{~d} t \int_{\mathbb{S}^{n-1}} \mathrm{~d} \mu_{\dot{\gamma}_{n-1}}\left\{\left(\frac{\partial(T \cdot \psi)}{\partial v^{*}}\right)^{2}+|\not \nabla T \cdot \psi|^{2}+(T \cdot \psi)^{2}\right\}\right|_{r=R_{j}^{\prime \prime}}\right\} \\
& \leq \frac{C(n, m, \delta, R)}{\left(\tau_{j}^{\prime \prime \prime}\right)^{3}}\left\{\int _ { \tau _ { 1 } + R ^ { * } } ^ { \infty } \mathrm { d } v ^ { * } \int _ { \mathbb { S } n - 1 } \mathrm { d } \mu _ { \dot { \gamma } _ { n - 1 } } \left\{r^{4-\delta}\left(\frac{\partial \chi}{\partial v^{*}}\right)^{2}+\sum_{k=0}^{3} r^{2}\left(\frac{\partial\left(T^{k} \cdot \psi\right)}{\partial v^{*}}\right)^{2}\right.\right. \\
& \left.+\sum_{k=0}^{2} \sum_{i=1}^{\frac{n(n-1)}{2}} r^{2}\left(\frac{\partial T^{k} \Omega_{i} \psi}{\partial v^{*}}\right)^{2}\right\}\left.\right|_{u^{*}=\tau_{1}} \\
& \left.+\int_{\Sigma_{\tau_{1}}}\left(\sum_{k=0}^{4} J^{N}\left(T^{k} \cdot \phi\right)+\sum_{k=0}^{3} \sum_{i=1}^{\frac{n(n-1)}{2}} J^{N}\left(T^{k} \Omega_{i} \phi\right), n\right)\right\} \\
& +C(n, m, R)\left\{\int_{\Sigma_{\tau_{2(2 j-1)-1}^{\prime \prime}}^{\tau_{2 j+1}^{\prime \prime \prime}}}\left(J^{T}(T \cdot \phi)+J^{T}\left(T^{2} \cdot \phi\right), n\right)\right.
\end{aligned}
$$

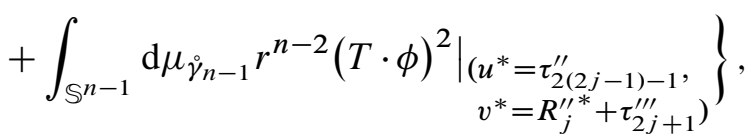

where in the last inequality we have used Proposition 4.22, and then chosen $R_{j}^{\prime \prime}(j \in \mathbb{N})$ suitably by Proposition 4.21; furthermore the inequality still holds if we add the integral of the nondegenerate energy on $R_{j}^{\prime \prime} \mathcal{P}_{\tau_{2 j-1}^{\prime \prime \prime}}^{\tau_{2 \prime \prime}^{\prime \prime \prime}}$ on the left-hand side and replace $J^{T}$ by $J^{N}$ in the first term of the integral on $\Sigma_{\tau_{2(2 j-1)-1}^{\tau_{2 j+1}^{\prime \prime \prime}}}^{\tau^{\prime \prime}}$ on the right-hand side. The last two terms on the right-hand side of (5-64) in fact decay with almost the same rate as the first; for first note here that we could have used Proposition 4.4 and Corollary 4.3 instead, and then employed Proposition 5.4 to obtain in any case that

$$
\begin{aligned}
& \int_{\tau_{2 j-1}^{\prime \prime \prime}}^{\tau_{2 j+1}^{\prime \prime \prime}} \mathrm{d} \tau \int_{\Sigma_{\tau}} \frac{1}{r^{\delta}}\left(J^{N}(T \cdot \phi), n\right) \\
& \leq \frac{C(n, m, \delta, R)}{\left(\tau_{2 j-1}^{\prime \prime \prime}\right)^{2}}\left\{\int _ { \tau _ { 1 } + R ^ { * } } ^ { \infty } \mathrm { d } v ^ { * } \int _ { \mathbb { S } ^ { n - 1 } } \mathrm { d } \mu _ { \dot { \gamma } _ { n - 1 } } \left\{r^{4-\delta}\left(\frac{\partial \chi}{\partial v^{*}}\right)^{2}+\sum_{k=0}^{3} r^{2}\left(\frac{\partial\left(T^{k} \cdot \psi\right)}{\partial v^{*}}\right)^{2}\right.\right. \\
& +\int_{\Sigma_{\tau_{1}}}\left(\sum_{k=0}^{4} J^{N}\left(T^{k} \cdot \phi\right)+\sum_{k=0}^{3} \frac{\left.\sum_{i=1}^{\frac{n(n-1)}{2}} \sum_{i=1}^{2} J^{2}\left(\frac{\partial T^{k} \Omega_{i} \psi}{\partial v^{*}}\right)^{2}\right\}\left.\right|_{u^{*}=\tau_{1}}}{\left.\left.\left(T^{k} \Omega_{i} \phi\right), n\right)\right\}}\right.
\end{aligned}
$$

It then follows that there exists a sequence $\tau_{j}^{\prime \prime \prime \prime} \in\left(\tau_{2 j-1}^{\prime \prime \prime}, \tau_{2 j+1}^{\prime \prime \prime}\right)$ such that 


$$
\begin{aligned}
& \int_{\Sigma_{\tau_{j}^{\prime \prime \prime \prime}} \tau_{2(j+2)+1}^{\prime \prime \prime}}\left(J^{N}(T \cdot \phi), n\right) \\
& \leq\left. r^{\delta}\right|_{\substack{\left(u^{*}=\tau_{j}^{\prime \prime \prime \prime}, v^{*}=R_{j+2}^{\prime \prime}{ }^{*}+\tau_{2(j+2)+1}^{\prime \prime \prime}\right)}} \int_{\Sigma_{\tau_{j}^{\prime \prime \prime \prime}}} \frac{1}{r^{\delta}}\left(J^{N}(T \cdot \phi), n\right) \\
& \leq \frac{C(n, m, \delta, R)}{\left(\tau_{j}^{\prime \prime \prime \prime}\right)^{3-\delta}}\left\{\int _ { \tau _ { 1 } + R ^ { * } } ^ { \infty } \mathrm { d } v ^ { * } \int _ { \mathbb { S } ^ { n - 1 } } \mathrm { d } \mu _ { \dot { \gamma } _ { n - 1 } } \left\{r^{4-\delta}\left(\frac{\partial \chi}{\partial v^{*}}\right)^{2}+\sum_{k=0}^{3} r^{2}\left(\frac{\partial\left(T^{k} \cdot \psi\right)}{\partial v^{*}}\right)^{2}\right.\right. \\
& \left.+\sum_{k=0}^{2} \sum_{i=1}^{\frac{n(n-1)}{2}} r^{2}\left(\frac{\partial T^{k} \Omega_{i} \psi}{\partial v^{*}}\right)^{2}\right\}\left.\right|_{u^{*}=\tau_{1}} \\
& \left.+\int_{\Sigma_{\tau_{1}}}\left(\sum_{k=0}^{4} J^{N}\left(T^{k} \cdot \phi\right)+\sum_{k=0}^{3} \sum_{i=1}^{\frac{n(n-1)}{2}} J^{N}\left(T^{k} \Omega_{i} \phi\right), n\right)\right\}
\end{aligned}
$$

because $\tau_{j}^{\prime \prime \prime \prime}\left(\tau_{2(j+2)+1}^{\prime \prime \prime}-\tau_{j}^{\prime \prime \prime \prime}\right)^{-1} \leq 1$. Secondly, the assumptions of Lemma 5.9 are satisfied in view of (5-58) on $u^{*}=\tau_{j}^{\prime \prime}(j \in \mathbb{N})$, which yields

$$
\begin{gathered}
\left.\int_{\mathbb{S}^{n-1}} \mathrm{~d} \mu_{\gamma_{n-1}} r^{n-2}(T \cdot \phi)^{2}\right|_{\left(u^{*}=\tau_{2(2 j-1)-1}^{\prime \prime}, v^{*}=R_{j}^{\prime \prime *}+\tau_{2 j+1}^{\prime \prime \prime}\right)} \\
\leq \frac{C(n, m, \delta, R)}{\left(\tau_{2 j-1}^{\prime \prime \prime}\right)^{3-\frac{\delta}{2}}}\left\{\int _ { \tau _ { 1 } + R ^ { * } } ^ { \infty } \mathrm { d } v ^ { * } \int _ { \mathbb { S } ^ { n - 1 } } \mathrm { d } \mu _ { \dot { \gamma } _ { n - 1 } } \left\{r^{4-\delta}\left(\frac{\partial \chi}{\partial v^{*}}\right)^{2}+\sum_{k=0}^{3} r^{2}\left(\frac{\partial T^{k} \cdot \psi}{\partial v^{*}}\right)^{2}\right.\right. \\
\left.+\sum_{k=0}^{2} \frac{n(n-1)}{\sum_{i=1}^{2}} r^{2}\left(\frac{\partial T^{k} \Omega_{i} \psi}{\partial v^{*}}\right)^{2}\right\}\left.\right|_{u^{*}=\tau_{1}} \\
\left.+\int_{\Sigma_{\tau_{1}}}\left(\sum_{k=0}^{4} J^{N}\left(T^{k} \cdot \phi\right)+\sum_{k=0}^{3} J^{N}\left(T^{k} \Omega_{i} \phi\right), n\right)\right\}
\end{gathered}
$$

because also

$$
\tau_{2 j-1}^{\prime \prime \prime}\left(\tau_{2 j+1}^{\prime \prime \prime}-\tau_{2(2 j-1)-1}^{\prime \prime}\right)^{-1} \leq C
$$

We shall now return to (5-64) — and its extension, which includes the nondegenerate energy on $R_{j}^{\prime \prime} \mathcal{P}_{\tau_{2 j}^{\prime \prime}-1}^{\tau_{2 j}^{\prime \prime \prime}}-$ to find that, after inserting (5-66) and using Proposition 5.2,

$$
\begin{aligned}
& \int_{\Sigma_{\tau_{2(2 j-1)-1}^{2 \prime \prime j+1}}^{\tau_{2 \prime \prime}^{\prime \prime}}}\left(J^{N}(T \cdot \phi)+J^{T}\left(T^{2} \cdot \phi\right), n\right) \\
& \leq C \int_{\Sigma_{\substack{\tau_{(2 j-1)-1}^{\prime \prime \prime} j \\
\tau_{(2 j-1)}^{\prime \prime \prime}}}}\left(J^{N}(T \cdot \phi)+J^{T}\left(T^{2} \cdot \phi\right), n\right) \\
& \leq C \int_{\substack{\Sigma_{\tau_{j-2}^{\prime \prime \prime \prime}} \\
\tau_{j, 1}^{\prime \prime \prime}+1}}\left(J^{N}(T \cdot \phi)+J^{T}\left(T^{2} \cdot \phi\right), n\right)
\end{aligned}
$$




$$
\begin{aligned}
\leq \frac{C(n, m, \delta, R)}{\left(\tau_{j-2}^{\prime \prime \prime \prime}\right)^{3-\delta}}\left\{\int _ { \tau _ { 1 } + R ^ { * } } ^ { \infty } \mathrm { d } v ^ { * } \int _ { \mathbb { S } ^ { n - 1 } } \mathrm { d } \mu _ { \dot { \gamma } _ { n - 1 } } \left\{r^{4-\delta}\left(\frac{\partial \chi}{\partial v^{*}}\right)^{2}+r^{4-\delta}\left(\frac{\partial(T \cdot \chi)}{\partial v^{*}}\right)^{2}+\sum_{k=0}^{4} r^{2}\left(\frac{\partial\left(T^{k} \cdot \psi\right)}{\partial v^{*}}\right)^{2}\right.\right. & \left.+\sum_{k=0}^{3} \sum_{i=1}^{\frac{n(n-1)}{2}} r^{2}\left(\frac{\partial T^{k} \Omega_{i} \psi}{\partial v^{*}}\right)^{2}\right\}\left.\right|_{u^{*}=\tau_{1}} \\
& \left.+\int_{\Sigma_{\tau_{1}}}\left(\sum_{k=0}^{5} J^{N}\left(T^{k} \cdot \phi\right)+\sum_{k=0}^{4} \sum_{i=1}^{\frac{n(n-1)}{2}} J^{N}\left(T^{k} \Omega_{i} \phi\right), n\right)\right\}
\end{aligned}
$$

and using (5-67), that there exists (another) sequence $\tau_{j}^{\prime \prime \prime \prime} \in\left(\tau_{2 j-1}^{\prime \prime \prime}, \tau_{2 j+1}^{\prime \prime \prime}\right)(j \in \mathbb{N})$ such that

$$
\begin{aligned}
& \int_{\Sigma_{\tau_{j}^{\prime \prime \prime \prime}}} \frac{1}{r^{\delta}}\left(J^{N}(T \cdot \phi), n\right) \\
& \leq \frac{C(n, m, \delta, R)}{\left(\tau_{j}^{\prime \prime \prime \prime}\right)^{4-\delta}}\left\{\int _ { \tau _ { 1 } + R ^ { * } } ^ { \infty } \mathrm { d } v ^ { * } \int _ { \mathbb { S } ^ { n - 1 } } \mathrm { d } \mu _ { \dot { \gamma } _ { n - 1 } } \left\{\sum_{k=0}^{1} r^{4-\delta}\left(\frac{\partial\left(T^{k} \cdot \chi\right)}{\partial v^{*}}\right)^{2}+\sum_{k=0}^{4} r^{2}\left(\frac{\partial\left(T^{k} \cdot \psi\right)}{\partial v^{*}}\right)^{2}\right.\right. \\
& +\int_{\Sigma_{\tau_{1}}}\left(\sum_{k=0}^{5} J^{N}\left(T^{k} \cdot \phi\right)+\sum_{k=0}^{\frac{n(n-1)}{2}} \sum_{i=1}^{4} \sum_{i=1}^{2}\left(\frac{\partial T^{k} \Omega_{i} \psi}{\partial v^{*}}\right)^{2}\right\}_{u^{*}=\tau_{1}} J_{\left.\left.\left(T^{k} \Omega_{i} \phi\right), n\right)\right\}}
\end{aligned}
$$

So for any $\tau>\tau_{1}$ we can choose $j \in \mathbb{N}$ such that $\tau \in\left(\tau_{2 j-1}^{\prime \prime \prime}, \tau_{2 j+1}^{\prime \prime \prime}\right)$ to obtain finally by Proposition 5.2 that

$$
\begin{aligned}
& \int_{\Sigma_{\tau} \cap\{r \leq R\}}\left(J^{N}(T \cdot \phi), n\right)
\end{aligned}
$$

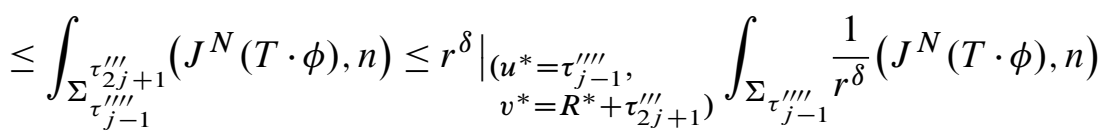

$$
\begin{aligned}
& \leq \frac{C(n, m, \delta, R)}{\tau^{4-2 \delta}}\left\{\int _ { \tau _ { 1 } + R ^ { * } } ^ { \infty } \mathrm { d } v ^ { * } \int _ { \mathbb { S } ^ { n - 1 } } \mathrm { d } \mu _ { \dot { \gamma } _ { n - 1 } } \left\{\sum_{k=0}^{1} r^{4-\delta}\left(\frac{\partial\left(T^{k} \cdot \chi\right)}{\partial v^{*}}\right)^{2}+\sum_{k=0}^{4} r^{2}\left(\frac{\partial\left(T^{k} \cdot \psi\right)}{\partial v^{*}}\right)^{2}\right.\right. \\
& \left.+\sum_{k=0}^{3} \sum_{i=1}^{\frac{n(n-1)}{2}} r^{2}\left(\frac{\partial T^{k} \Omega_{i} \psi}{\partial v^{*}}\right)^{2}\right\}\left.\right|_{u^{*}=\tau_{1}} \\
& \left.+\int_{\Sigma_{\tau_{1}}}\left(\sum_{k=0}^{5} J^{N}\left(T^{k} \cdot \phi\right)+\sum_{k=0}^{4} \sum_{i=1}^{\frac{n(n-1)}{2}} J^{N}\left(T^{k} \Omega_{i} \phi\right), n\right)\right\} .
\end{aligned}
$$

Remark 5.11. For the removal of the restriction to dyadic sequences in the last step of the proof, (5-69) and (5-70), we could have equally obtained a decay estimate for the energy flux through $\Sigma_{\tau} \cap\left\{r^{*} \leq R^{*}+\tau^{k}\right\}$ (with $k \in \mathbb{N}$ ) by replacing $\Sigma_{\tau_{j-1}^{\prime \prime \prime \prime}}^{\tau_{2 \prime \prime}^{\prime \prime \prime}}$ by $\Sigma_{\tau_{j-1}^{\prime \prime \prime \prime}}^{\tau_{j \prime \prime}^{\prime \prime \prime}+\tau^{k}}$ in the first estimate in (5-70); if $\delta>0$ for a chosen $k \in \mathbb{N}$ 
is restricted to $\delta<(1+k)^{-1}$ we then still obtain a decay rate of $\tau^{4-(1+k) \delta}$ for the energy flux through $\Sigma_{\tau} \cap\left\{r^{*} \leq R^{*}+\tau^{k}\right\}$.

\section{Pointwise bounds}

In this section we first prove pointwise estimates on $|\phi|$ and $\left|\partial_{t} \phi\right|$ separately based on the energy decay results Propositions 5.4 and 5.6. Then we give the interpolation argument to improve the pointwise decay on $|\phi|$. As we shall see in view of the nondegenerate energy estimates of Section 5 we may restrict ourselves in the first place to a radial region away from the horizon. Recall the definition (4-3) of $\Sigma_{\tau}$ $\left(r_{1} \doteq R>\sqrt[n-2]{8 n m}\right)$.

Proposition 6.1 (pointwise decay). (i) Let $\phi$ be a solution of the wave equation (1-1), with initial data on $\Sigma_{\tau_{0}}\left(\tau_{0}>0\right)$ such that

$$
\left.D \doteq \int_{\tau_{0}+R^{*}}^{\infty} \mathrm{d} v^{*} \int_{\mathbb{S}^{n-1}} \mathrm{~d} \mu_{\dot{\gamma}_{n-1}} \sum_{k=0}^{\left[\frac{n}{2}\right]+1} r^{2}\left(\frac{\partial T^{k} \cdot \psi}{\partial v^{*}}\right)^{2}\right|_{u^{*}=\tau_{0}}+\int_{\Sigma_{\tau_{0}}}\left(\sum_{k=0}^{\left[\frac{n}{2}\right]+2} J^{N}\left(T^{k} \cdot \phi\right), n\right)<\infty .
$$

Then there is a constant $C(n, m)$ such that, for $r_{0}<r<R$,

$$
|\phi(t, r)| \leq \frac{C(n, m) \sqrt{D}}{\tau} \quad\left(\tau=\frac{1}{2}\left(t-R^{*}\right)>\tau_{0}\right) .
$$

(ii) If, moreover, the initial data satisfies

$$
\begin{aligned}
D \doteq \int_{\tau_{0}+R^{*}}^{\infty} \mathrm{d} v^{*} \int_{\mathbb{S}^{n-1}} \mathrm{~d} \mu_{\dot{\gamma}_{n-1}}\left\{\sum_{k=0}^{\left[\frac{n}{2}\right]+1} r^{4-\delta}\left(\frac{\partial\left(T^{k} \cdot \chi\right)}{\partial v^{*}}\right)^{2}\right. \\
\left.\quad+\sum_{k=0}^{\left[\frac{n}{2}\right]+4} r^{2}\left(\frac{\partial\left(T^{k} \cdot \psi\right)}{\partial v^{*}}\right)^{2}+\sum_{k=0}^{\left[\frac{n}{2}\right]+3} \sum_{i=1}^{\frac{n(n-1)}{2}} r^{2}\left(\frac{\partial T^{k} \Omega_{i} \psi}{\partial v^{*}}\right)^{2}\right\}\left.\right|_{u^{*}=\tau_{0}} \\
+\int_{\Sigma_{\tau_{0}}}\left(\sum_{k=0}^{\left[\frac{n}{2}\right]+5} J^{N}\left(T^{k} \cdot \phi\right)+\sum_{k=0}^{\left[\frac{n}{2}\right]+4} \frac{n(n-1)}{2} \sum_{i=1}^{2} J^{N}\left(T^{k} \Omega_{i} \phi\right), n\right)<\infty \quad(6-3)
\end{aligned}
$$

for some $0<\delta<\frac{1}{4}$, and $R>\sqrt[n-2]{\frac{8 n m}{\delta}}$, then there is a constant $C(n, m, \delta, R)$ such that for $r_{0}<r<R$,

$$
\left|\partial_{t} \phi(t, r)\right| \leq \frac{C \sqrt{D}}{\tau^{2-2 \delta}} \quad\left(\tau=\frac{1}{2}\left(t-R^{*}\right)>\tau_{0}\right)
$$

The pointwise bounds are obtained from the energy estimates of Section 5 using Sobolev inequalities and elliptic estimates; the former provide the link between pointwise and integral quantities, and the latter allow for the expression of these integral quantities in terms of higher-order energies.

Sobolev embedding. By the extension theorem applied to the Sobolev embedding $\mathrm{H}^{s}\left(\mathbb{R}^{n}\right) \subset \mathrm{L}^{\infty}\left(\mathbb{R}^{n}\right)$ $\left(s>\frac{n}{2}\right.$ ) we have, for $r_{0}<\bar{r}<R$, 


$$
|\phi(\bar{t}, \bar{r})|^{2} \leq\left. C(n) \int_{r_{0}^{*}}^{R^{*}} \mathrm{~d} r^{*} \int_{\mathbb{S}^{n-1}} \mathrm{~d} \mu_{\dot{\gamma}_{n-1}}\left\{\phi^{2}+\sum_{|\alpha| \geq 1}^{|\alpha| \leq\left[\frac{n}{2}\right]+1}\left|\bar{\nabla}^{\alpha} \phi\right|^{2}\right\} r^{n-1}\right|_{t=\bar{t}},
$$

where $\bar{\nabla}$ denote the tangential derivatives to the hypersurface $\bar{\Sigma}_{t}$, and $\alpha$ denotes a multiindex of order $n$. Elliptic estimates. Note that for any solution $\phi$ of the wave equation we have

$$
T^{2} \cdot \phi=\frac{\partial^{2} \phi}{\partial r^{* 2}}+\left(1-\frac{2 m}{r^{n-2}}\right) \frac{n-1}{r} \frac{\partial \phi}{\partial r^{*}}+\left(1-\frac{2 m}{r^{n-2}}\right) \phi_{r^{2} \dot{\gamma}_{n-1}} \phi \doteq L \cdot \phi,
$$

where the operator

$$
L=\left(1-\frac{2 m}{r^{n-2}}\right) \bar{g}^{i j} \bar{\nabla}_{i} \partial_{j}
$$

is clearly elliptic. (Here $\bar{g}_{t}=\left.g\right|_{\bar{\Sigma}_{t}}$ denotes the restriction of $g$ to the spacelike hypersurfaces $\bar{\Sigma}_{t}$, a Riemannian metric on $\bar{\Sigma}_{t}$, and $i, j=1, \ldots, n$.) In view of the standard higher-order interior elliptic regularity estimate

$$
\|\phi\|_{\mathrm{H}^{m+2}\left(\widehat{\Sigma}_{t}\right)} \leq C\left(\|L \cdot \phi\|_{\mathrm{H}^{m}\left(\widehat{\Sigma}_{t}\right)}+\|\phi\|_{\mathrm{L}^{2}\left(\widehat{\Sigma}_{t}\right)}\right), \quad \widehat{\Sigma}_{t} \doteq \bar{\Sigma}_{t} \cap\left\{r_{0}<r<R\right\},
$$

we conclude with (6-5) that, in the case where $\left[\frac{n}{2}\right]+1$ is even,

$$
|\phi|^{2} \leq C(n, m) \int_{r_{0}^{*}}^{R^{*}} \mathrm{~d} r^{*} \int_{\mathbb{S}^{n-1}} \mathrm{~d} \mu_{\dot{\gamma}_{n-1}} \sum_{l=0}^{\left[\frac{n}{2}\right]+1}\left(T^{l} \cdot \phi\right)^{2} r^{n-1}
$$

in general we have:

Lemma 6.2 (pointwise estimate in terms of higher-order energies). Let $\phi$ be a solution of the wave equation (1-1), and $n \geq 3$. Then there exists a constant $C(n, m)$ such that, for all $r_{0}<r<R$,

$$
|\phi(t, r)|^{2} \leq C(n, m)\left[\|\phi\|_{\mathrm{L}^{2}\left(\widehat{\Sigma}_{t}\right)}^{2}+\int_{\widehat{\Sigma}_{t}} \sum_{l=0}^{\left[\frac{n}{2}\right]}\left(J^{T}\left(T^{l} \cdot \phi\right), n\right)\right] .
$$

Proof of Proposition 6.1. In view of the Lemma 6.2 and the energy decay estimates of Section 5 it remains to control the zeroth order term $\|\phi\|_{\mathrm{L}^{2}\left(\widehat{\Sigma}_{t}\right)}$; we multiply the integrand by $\left(\frac{R}{r}\right)^{2} \geq 1$ and extend the integral to $u^{*}=\tau=\frac{1}{2}\left(t-R^{*}\right), v^{*} \geq \frac{1}{2}\left(t+R^{*}\right)$.

(i) By Lemma C.2 we can then estimate $\|\phi\|_{\mathrm{L}^{2}\left(\widehat{\Sigma}_{t}\right)}^{2}$ by the energy flux through $\Sigma_{\tau=\frac{1}{2}\left(t-R^{*}\right)}$, and apply Proposition 5.4 to the higher-order energies of Lemma 6.2.

(ii) Here we extend the integral only to $\tau+R^{*} \leq v^{*} \leq \tau+R^{*}+\tau^{3}$ and apply Lemma C.4 to obtain

$$
\begin{aligned}
\int_{r_{0}^{*}}^{R^{*}} \mathrm{~d} r^{*} \int_{\mathbb{S}^{n-1}} \mathrm{~d} \mu_{\dot{\gamma}_{n-1}}\left(\partial_{t} \phi\right)^{2} r^{n-1} \leq & C(n, m) R^{2} \int_{\Sigma_{\tau} \cap\left\{r^{*} \leq R^{*}+\tau^{3}\right\}}\left(J^{T}\left(\partial_{t} \phi\right), n\right) \\
& +\left.C(n, m) \frac{R^{2}}{r} \int_{\mathbb{S}^{n-1}} r^{n-1}\left(\partial_{t} \phi\right)^{2}\right|_{\left(u^{*}=\tau, v^{*}=\tau+R^{*}+\tau^{3}\right)} .
\end{aligned}
$$


As in the proof of Lemma 5.9 we obtain by integrating from infinity and Cauchy's inequality that

$$
\int_{\mathbb{S}^{n-1}} \mathrm{~d} \mu_{\hat{\gamma}_{n-1}} r^{n-2}\left(\partial_{t} \phi\right)^{2}\left(\tau, \tau+R^{*}\right) \leq \frac{C(n, m)}{1-\frac{2 m}{R^{n-2}}} \int_{\Sigma_{\tau}}\left(J^{T}\left(\partial_{t} \phi\right), n\right),
$$

which decays by Proposition 5.4 with a rate $\tau^{-2}$. Moreover, as in the proof of Lemma 5.9,

$$
\begin{aligned}
& \left.\int_{\mathbb{S}^{n-1}} \mathrm{~d} \mu_{\dot{\gamma}_{n-1}} r^{n-1}\left(\partial_{t} \phi\right)^{2}\right|_{\left(u^{*}=\tau, v^{*}=\tau+R^{*}+\tau^{3}\right)} \\
& \quad=\left.\int_{\mathbb{S}^{n-1}} \mathrm{~d} \mu_{\dot{\gamma}_{n-1}} r^{n-1}\left(\partial_{t} \phi\right)^{2}\right|_{\left(u^{*}=\tau, v^{*}=\tau+R^{*}\right)}+\left.\int_{\tau+R^{*}}^{\tau+R^{*}+\tau^{3}} \mathrm{~d} v^{*} \int_{\mathbb{S}^{n-1}} \mathrm{~d} \mu_{\dot{\gamma}_{n-1}} 2 \partial_{t} \psi \frac{\partial \partial_{t} \psi}{\partial v^{*}}\right|_{u^{*}=\tau}
\end{aligned}
$$

and

$$
\begin{aligned}
\int_{\tau+R^{*}}^{\tau+R^{*}+\tau^{3}} \mathrm{~d} v^{*} & \left.\int_{\mathbb{S}^{n-1}} \mathrm{~d} \mu_{\gamma_{n-1}} \partial_{t} \psi \frac{\partial \partial_{t} \psi}{\partial v^{*}}\right|_{u^{*}=\tau} \\
& \leq \sqrt{\int_{\tau+R^{*}}^{\infty} \int_{\mathbb{S}^{n-1}} \mathrm{~d} \mu_{\dot{\gamma}_{n-1}} \frac{1}{r^{2}}\left(\partial_{t} \phi\right)^{2} r^{n-1}} \times \sqrt{\int_{\tau+R^{*}}^{\infty} \int_{\mathbb{S}^{n-1}} \mathrm{~d} \mu_{\dot{\gamma}_{n-1}} r^{2}\left(\frac{\partial r^{\frac{n-1}{2}} \partial_{t} \phi}{\partial v^{*}}\right)^{2}},
\end{aligned}
$$

the first factor decaying with a rate $\tau^{-1}$ by Lemma C.2 and Proposition 5.4, and the second factor bounded by the weighted energy inequality for $r^{\frac{n-1}{2}} \partial_{t} \phi$ in place of $\psi$ with $p=2$. Therefore

$$
\left.\int_{\mathbb{S}^{n-1}} r^{n-1}\left(\partial_{t} \phi\right)^{2}\right|_{\left(u^{*}=\tau, v^{*}=\tau+R^{*}+\tau^{3}\right)} \leq \frac{C(n, m)}{1-\frac{2 m}{R^{n-2}}} \frac{D}{\tau} .
$$

By virtue of Proposition 5.6 (compare in particular Remark 5.11 on page 587), the first term on the right-hand side of (6-11) decays with a rate of $\tau^{4-4 \delta}$, and this is matched by the second term in view of the prefactor $r^{-1}=\left(R^{*}+\tau^{3}\right)^{-1}$, which is the result of our choice of powers of $\tau$ in the extension of the integral Lemma 6.2 applied to the solution $\partial_{t} \phi$ of (1-1) then yields the pointwise decay result (6-4) after having applied Proposition 5.6 to the higher-order energies on the right-hand side of (6-10).

Interpolation. We shall now interpolate between the results (i) and (ii) of Proposition 6.1 to improve the pointwise estimate for $|\phi|$. Our argument can in some sense be compared to the proof of improved decay in [Luk 2010]. The basic observation underlying this argument is that, for $r_{0}<r<R$ and $t_{1}>t_{0}$,

$$
\begin{aligned}
r^{n-2} \phi^{2}\left(r, t_{1}\right) & =r^{n-2} \phi^{2}\left(r, t_{0}\right)+\int_{t_{0}}^{t_{1}} 2 \phi(t, r) \frac{\partial \phi}{\partial t}(t, r) r^{n-2} \mathrm{~d} t \\
& \leq r^{n-2} \phi^{2}\left(r, t_{0}\right)+\frac{1}{t_{0}^{1-2 \delta}} \int_{t_{0}}^{t_{1}} \phi^{2}(t, r) r^{n-2} \mathrm{~d} t+t_{0}^{1-2 \delta} \int_{t_{0}}^{t_{1}}\left(\frac{\partial \phi}{\partial t}\right)^{2}(t, r) r^{n-2} \mathrm{~d} t .
\end{aligned}
$$

Moreover, as a consequence of Lemma 6.3,

$$
r^{n-2} \phi^{2}(t, r) \leq R^{n-2} \phi^{2}(t, R)+\left(1-\frac{2 m}{r_{0}^{n-2}}\right)^{-1} \int_{r^{*}}^{R^{*}}\left(\frac{\partial \phi}{\partial r^{*}}\right)^{2} r^{n-1} \mathrm{~d} r^{*},
$$


we obtain an estimate for the timelike integrals in terms of the corresponding integrals at $r=R$ and spacetime integrals, using the Sobolev inequality on the sphere:

$$
\begin{aligned}
\int_{t_{0}}^{t_{1}} r^{n-2} \phi^{2}(t, r) \mathrm{d} t \leq & \int_{t_{0}}^{t_{1}} \mathrm{~d} t \int_{\mathbb{S}^{n-1}} \mathrm{~d} \mu_{\dot{\gamma}_{n-1}} \sum_{|\alpha| \leq\left[\frac{n}{2}\right]+1} R^{n-2}\left(\Omega^{\alpha} \phi\right)^{2}(t, R) \\
& +\left(1-\frac{2 m}{r_{0}^{n-2}}\right)^{-1} \int_{t_{0}}^{t_{1}} \mathrm{~d} t \int_{r^{*}}^{R^{*}} \mathrm{~d} r^{*} \int_{\mathbb{S}^{n-1}} \mathrm{~d} \mu_{\dot{\gamma}_{n-1}} r^{n-1} \sum_{|\alpha| \leq\left[\frac{n}{2}\right]+1}\left(\frac{\partial \Omega^{\alpha} \phi}{\partial r^{*}}\right)^{2}(t, r) .
\end{aligned}
$$

Lemma 6.3. Let $a<b \in \mathbb{R}$ and $\phi \in \mathrm{C}^{1}([a, b])$. Then, for all $n \geq 3$,

$$
a^{n-2} \phi^{2}(a) \leq b^{n-2} \phi^{2}(b)+\int_{a}^{b}\left(\frac{\mathrm{d} \phi}{\mathrm{d} x}\right)^{2} x^{n-1} \mathrm{~d} x .
$$

Proof. Since, by integration by parts,

$$
\int_{a}^{b} 2 \phi(x) \frac{\mathrm{d} \phi}{\mathrm{d} x}(x) x^{n-2} \mathrm{~d} x=\left.2 \phi^{2}(x) x^{n-2}\right|_{a} ^{b}-\int_{a}^{b} 2 \phi(x) \frac{\mathrm{d} \phi}{\mathrm{d} x}(x) x^{n-2} \mathrm{~d} x-\int_{a}^{b} 2 \phi^{2}(x)(n-2) x^{n-3} \mathrm{~d} x,
$$

it clearly follows, with Cauchy's inequality,

$$
a^{n-2} \phi^{2}(a) \leq b^{n-2} \phi^{2}(b)+\int_{a}^{b}\left(\frac{\mathrm{d} \phi}{\mathrm{d} x}\right)^{2} x^{n-1} \mathrm{~d} x+[1-(n-2)] \int_{a}^{b} \frac{1}{x^{2}} \phi^{2}(x) x^{n-1} \mathrm{~d} x .
$$

Proposition 6.4 (improved interior pointwise decay). Let $\phi$ be a solution of the wave equation (1-1), with initial data on $\Sigma_{\tau_{0}}\left(\tau_{0}>1\right)$ satisfying

$$
\begin{aligned}
D \doteq \int_{\tau_{0}+R^{*}}^{\infty} \mathrm{d} v^{*} & \int_{\mathbb{S}^{n-1}} \mathrm{~d} \mu_{\dot{\gamma}_{n-1}}\left\{\sum_{k=0}^{2} \sum_{|\alpha| \leq\left[\frac{n}{2}\right]+1} r^{4-\delta}\left(\frac{\partial\left(T^{k} \cdot \Omega^{\alpha} \chi\right)}{\partial v^{*}}\right)^{2}\right. \\
& \left.+\sum_{k=0}^{5} \sum_{|\alpha| \leq\left[\frac{n}{2}\right]+1} r^{2}\left(\frac{\partial T^{k} \Omega^{\alpha} \psi}{\partial v^{*}}\right)^{2}+\sum_{k=0}^{4} \sum_{|\alpha| \leq\left[\frac{n}{2}\right]+2} r^{2}\left(\frac{\partial T^{k} \Omega^{\alpha} \psi}{\partial v^{*}}\right)^{2}\right\}\left.\right|_{u^{*}=\tau_{0}} \\
& +\int_{\Sigma_{\tau_{0}}}\left(\sum_{k=0}^{6} \sum_{|\alpha| \leq\left[\frac{n}{2}\right]+1} J^{N}\left(T^{k} \Omega^{\alpha} \phi\right)+\sum_{k=0} \sum_{|\alpha| \leq\left[\frac{n}{2}\right]+2} J^{N}\left(T^{k} \Omega^{\alpha} \phi\right), n\right)<\infty
\end{aligned}
$$

for some $0<\delta<\frac{1}{4}$, where $R>\sqrt[n-2]{\frac{8 n m}{\delta}}, n \geq 3$. Then there exists a constant $C(n, m, \delta, R)$ such that, for $\sqrt[n-2]{2 m}<r_{0}<r<R$

$$
r^{\frac{n-2}{2}}|\phi|(t, r) \leq \frac{C D}{t^{\frac{3}{2}-\delta}} .
$$

Proof. Let $\bar{t}_{0}=2\left(\tau_{0}+\tau_{0}\right)+R^{*}$ and $\bar{t}_{1}=\bar{t}_{0}+2 \tau_{0}$. Then by (6-18), Proposition 4.4 and Proposition 4.1,

$$
\int_{\bar{t}_{0}}^{\bar{t}_{1}} \phi^{2}(t, r) r^{n-2} \mathrm{~d} t \leq C(n, m, R) \int_{\Sigma_{2 \tau_{0}}}\left(\sum_{k=0}^{1} \sum_{|\alpha| \leq\left[\frac{n}{2}\right]+1} J^{T}\left[T^{k} \Omega^{\alpha} \phi\right], n\right) ;
$$


hence by Proposition 5.4 there exists $t_{0}^{\prime} \in\left(\bar{t}_{0}, \bar{t}_{1}\right)$ such that

$$
r^{n-2} \phi^{2}\left(t_{0}^{\prime}, r\right) \leq \frac{C(n, m, R) D}{\bar{t}_{0}^{3}} .
$$

Now set $\tau_{0}^{\prime}=\frac{1}{2}\left(t_{0}^{\prime}-R^{*}\right)$ and $\tau_{j}^{\prime}=2 \tau_{j-1}^{\prime}(j \in \mathbb{N})$, and $t_{j}^{\prime}=2 \tau_{j}^{\prime}+R^{*}(j \in \mathbb{N})$; note that $t_{j+1}^{\prime}-t_{j}^{\prime}=\frac{1}{2}\left(t_{j}^{\prime}-R^{*}\right)$. Now consider (6-16) with $t_{1}=t_{j+1}^{\prime}, t_{0}=t_{j}^{\prime}$; since by (6-18), together with Propositions 4.1 and 4.4,

$$
\int_{t_{j}^{\prime}}^{t_{j+1}^{\prime}} r^{n-2} \phi^{2}(t, r) \mathrm{d} t \leq C(n, m, R) \int_{\Sigma_{\tau_{j}^{\prime}}}\left(\sum_{k=0}^{1} \sum_{|\alpha| \leq\left[\frac{n}{2}\right]+1} J^{T}\left[T^{k} \Omega^{\alpha} \phi\right], n\right),
$$

and by Propositions 4.21 and 4.22 ,

$$
\begin{aligned}
\int_{t_{j}^{\prime}}^{t_{j+1}^{\prime}} r^{n-2}\left(\partial_{t} \phi\right)^{2}(t, r) \mathrm{d} t \leq & C(n, m, R)\left\{\int_{\Sigma_{\tau_{j}^{\prime}} \cap\left\{r^{*} \leq R^{*}+\left(\tau_{j}^{\prime}\right)^{3}\right\}}\left(\sum_{k=1}^{2} \sum_{|\alpha| \leq\left[\frac{n}{2}\right]+1} J^{T}\left[T^{k} \Omega^{\alpha} \phi\right], n\right)\right. \\
& +\left.\int_{\mathbb{S}^{n-1}} \mathrm{~d} \mu_{\hat{\gamma}_{n-1}} \sum_{|\alpha| \leq\left[\frac{n}{2}\right]+1} r^{n-2}\left(\Omega^{\alpha} \partial_{t} \phi\right)^{2}\right|_{\left.\left(u^{*}=\tau_{j}^{\prime}, v^{*}=R^{*}+\tau_{j}^{\prime}+\left(\tau_{j}^{\prime}\right)^{3}\right)\right\}}
\end{aligned}
$$

which decays with the rate $\tau^{4-4 \delta}$ as is shown in the proof of Proposition 6.1(ii), we obtain

$$
\begin{aligned}
r^{n-2} \phi^{2}\left(r, t_{j+1}^{\prime}\right) & \leq r^{n-2} \phi^{2}\left(r, t_{j}^{\prime}\right)+\frac{C(n, m, R)}{\left(t_{j}^{\prime}\right)^{1-2 \delta}} \frac{D}{\left(\tau_{j}^{\prime}\right)^{2}}+C(n, m, \delta, R)\left(t_{j}^{\prime}\right)^{1-2 \delta} \frac{D}{\left(\tau_{j}^{\prime}\right)^{4-4 \delta}} \\
& \leq r^{n-2} \phi^{2}\left(r, t_{j}^{\prime}\right)+\frac{C(n, m, \delta, R) D}{\left(t_{j}^{\prime}\right)^{3-2 \delta}} .
\end{aligned}
$$

In fact, by induction on $j \in \mathbb{N}$ using (6-23) for $j=0$, we have shown

$$
r^{n-2} \phi^{2}\left(r, t_{j}^{\prime}\right) \leq \frac{C(n, m, \delta, R) D}{\left(t_{j}^{\prime}\right)^{3-2 \delta}} \quad(j \in \mathbb{N} \cup\{0\}) .
$$

Finally for any $t \geq t_{0}^{\prime}$ we may choose $j \in \mathbb{N} \cup\{0\}$ such that $t \in\left(t_{j}^{\prime}, t_{j+1}^{\prime}\right)$ and conclude the proof by applying (6-27) and (6-26), which holds with $t$ in place of $t_{j+1}^{\prime}$.

Extension to the horizon. Note that for $\sqrt[n-2]{2 m} \leq r<r_{0}$, the same interpolation (6-16) by integration along lines of constant radius $r<r_{0}$ can be carried out. However, on the right-hand sides of (6-17) and (6-18) a new term results from the integration on $v^{*}=\frac{1}{2}\left(t_{0}+r_{0}^{*}\right)$ from the radius $r<r_{0}$ to $r=r_{0}$; but we infer from the explicit construction (3-19) that the resulting integrand

$$
\left(\frac{2}{1-\frac{2 m}{r^{n-2}}} \frac{\partial \phi}{\partial u^{*}}\right)^{2} \leq T[\phi](Y, Y) \leq\left(J^{N}[\phi], N\right)
$$

is controlled by Corollary 4.3, and the proof of Proposition 6.4 above extends to that of Theorem 2 by replacing $J^{T}$ by $J^{N}$ on the right-hand sides of (6-22), (6-24) and (6-25). 


\section{Appendix A: Notation}

Contraction. We sum over repeated indices. Also we use interchangeably

$$
g(V, N) \doteq(V, N) \doteq V_{\mu} N^{\mu}, \quad J \cdot N \doteq(J, N) \doteq J_{\mu} N^{\mu},
$$

where $V, N$ are vector fields, and $J$ is a 1 -form.

Integration. Let $\mathcal{D}$ in $\mathcal{M}$ be a domain bounded by two homologous hypersurfaces, $\Sigma_{1}$ and $\Sigma_{2}$ being its past and future boundary, respectively. We then write $\int_{\Sigma_{1}}(J, n)$ for the boundary terms on $\Sigma_{1}$ arising from a general current $J$ in the expression $\int_{\partial \mathcal{D}}{ }^{*} J$. If $\mathcal{S} \subset \Sigma_{1}$ is spacelike, then $(J, n)=g(J, n)$ is in fact the inner product of $J$ with the timelike normal $n$ to $\Sigma_{1}$; e.g., on constant $t$-slices $\bar{\Sigma}_{t}$ (see Section 2) we have $n=\left(1-\frac{2 m}{r^{n-2}}\right)^{-\frac{1}{2}} \frac{\partial}{\partial t}$. If $\mathcal{U} \subset \Sigma_{1}$ is an outgoing null segment then $\int_{\mathcal{U}}(J, n)$ denotes an integral of the form $\int \mathrm{d} v \int_{\mathrm{S}} \mathrm{d} \mu_{\gamma} g\left(J, \frac{\partial}{\partial v}\right)$; e.g., on the outgoing null segments of the hypersurfaces $\Sigma_{\tau}$ (see Section 4), we have

$$
\int_{\Sigma_{\tau} \cap\{r \geq R\}}(J, n) \doteq \int_{\tau+R^{*}}^{\infty} \mathrm{d} v^{*} \int_{\mathbb{S}^{n-1}} \mathrm{~d} \mu_{\dot{\gamma}_{n-1}} r^{n-1}\left(J, \frac{\partial}{\partial v^{*}}\right) .
$$

The volume form is usually omitted:

$$
\int_{\mathcal{D}} f \doteq \int_{\mathcal{D}} f \mathrm{~d} \mu_{g} \quad(\mathcal{D} \subset \mathcal{M})
$$

\section{Appendix B: Formulas for reference}

In this appendix we summarize a few formulas for reference.

The wave equation. The d'Alembert operator in (1-1) can we written out in any coordinate system according to

$$
\square_{g} \phi=\left(g^{-1}\right)^{\mu \nu} \nabla_{\mu} \partial_{\nu} \phi,
$$

where $\nabla$ denotes the covariant derivative of the Levi-Civita connection of $g$.

Components of the energy momentum tensor. The components of the energy momentum tensor

$$
T_{\mu \nu}(\phi)=\partial_{\mu} \phi \partial_{\nu} \phi-\frac{1}{2} g_{\mu \nu} \partial^{\alpha} \phi \partial_{\alpha} \phi
$$

tangential to $\mathcal{Q}$ are given in $\left(u^{*}, v^{*}\right)$-coordinates by

$$
T_{u^{*} u^{*}}=\left(\frac{\partial \phi}{\partial u^{*}}\right)^{2}, \quad T_{v^{*} v^{*}}=\left(\frac{\partial \phi}{\partial v^{*}}\right)^{2}, \quad T_{u^{*} v^{*}}=\left(1-\frac{2 m}{r^{n-2}}\right)|\not \phi|_{r^{2} \dot{\gamma}_{n-1}}^{2} .
$$

We also refer to (B-2) as the null decomposition of the energy momentum tensor. Note here that

$$
\begin{gathered}
\partial^{\alpha} \phi \partial_{\alpha} \phi=-\frac{1}{1-\frac{2 m}{r^{n-2}}}\left(\frac{\partial \phi}{\partial u^{*}}\right)\left(\frac{\partial \phi}{\partial v^{*}}\right)+|\not \nabla \phi|_{r^{2} \dot{\gamma}_{n-1}}^{2}, \\
\frac{1}{r^{2}} \stackrel{\gamma}{n-1}_{n B}^{A B} T_{A B}=|\not \nabla \phi|_{r^{2} \dot{\gamma}_{n-1}}^{2}-\frac{1}{2}(n-1) \partial^{\alpha} \phi \partial_{\alpha} \phi .
\end{gathered}
$$


Integration. A typical domain of integration that we use is

$$
{ }^{R} \mathcal{D}_{\tau_{1}}^{\tau_{2}}=\left\{\left(u^{*}, v^{*}\right): \tau_{1} \leq u^{*} \leq \tau_{2}, v^{*}-u^{*} \geq R^{*}\right\} .
$$

In local coordinates we have, by calculating the volume form from (2-20), that

$$
\int_{R_{\mathcal{D}_{\tau_{1}}}^{\tau_{2}}} \mathrm{~d} \mu_{g}=\int_{\tau_{1}}^{\tau_{2}} \mathrm{~d} u^{*} \int_{u^{*}+R^{*}}^{\infty} \mathrm{d} v^{*} \int_{\mathbb{S}^{n-1}} \mathrm{~d} \mu_{\dot{\gamma}_{n-1}} 2\left(1-\frac{2 m}{r^{n-2}}\right) r^{n-1} .
$$

For a general current $J$ the energy identity on this domain reads

$$
\int_{R \mathcal{D}_{\tau_{1}}^{\tau_{2}}} K^{X} \mathrm{~d} \mu_{g}=\int_{\partial^{R} \mathcal{D}_{\tau_{1}}^{\tau_{2}}}{ }^{*} J
$$

where the right-hand side is given more explicitly by

$$
\begin{aligned}
& \int_{\partial R \mathcal{D}_{\tau_{1}}^{\tau_{2}}}^{*} J \\
& =-\left.\int_{R^{*}+\tau_{2}}^{\infty} \mathrm{d} v^{*} \int_{\mathbb{S}^{n-1}} \mathrm{~d} \mu_{\dot{\gamma}_{n-1}} r^{n-1} g\left(J, \frac{\partial}{\partial v^{*}}\right)\right|_{u^{*}=\tau_{2}}-\left.\int_{\tau_{1}}^{\tau_{2}} \mathrm{~d} u^{*} \int_{\mathbb{S}^{n-1}} \mathrm{~d} \mu_{\dot{\gamma}_{n-1}} r^{n-1} g\left(J, \frac{\partial}{\partial u^{*}}\right)\right|_{v^{*} \rightarrow \infty} \\
& \quad+\left.\int_{R^{*}+\tau_{1}}^{\infty} \mathrm{d} v^{*} \int_{\mathbb{S}^{n-1}} \mathrm{~d} \mu_{\dot{\gamma}_{n-1}} r^{n-1} g\left(J, \frac{\partial}{\partial v^{*}}\right)\right|_{u^{*}=\tau_{1}}-\left.\int_{R^{*}+2 \tau_{1}}^{R^{*}+2 \tau_{2}} \mathrm{~d} t \int_{\mathbb{S}^{n-1}} r^{n-1} g\left(J, \frac{\partial}{\partial r^{*}}\right)\right|_{r=R} .(\mathrm{B}-6)
\end{aligned}
$$

Radial functions. In this appendix we summarize some statements on the relation between $r$ and

$$
r^{*}=\int_{(n m)^{\frac{1}{n-2}}}^{r} \frac{1}{1-\frac{2 m}{r^{n-2}}} \mathrm{~d} r .
$$

The proofs are omitted here, but can be found in [Schlue 2012].

Proposition B.1. For all $n \geq 3$,

$$
\lim _{\frac{r}{n-2} \sqrt{2 m}} \frac{r^{*}}{r}=1 .
$$

While this fact concerns the region $r^{*} \geq 0$ and is essentially due to $\lim _{x \rightarrow \infty} \frac{\log x}{x}=0$, the next concerns $r^{*} \leq 0$ and is similarly due to $\lim _{x \rightarrow 0} x \log x=0$.

Proposition B.2. For all $n \geq 3$,

$$
\lim _{\frac{r}{n-2} \sqrt{2 m}} \rightarrow 1\left(1-\frac{2 m}{r^{n-2}}\right)\left(-r^{*}\right)=0 .
$$

In fact we have:

Proposition B.3. For $r^{*}<0$,

$$
\left(1-\frac{2 m}{r^{n-2}}\right) \leq \frac{(2 m)^{\frac{1}{n-2}}}{\left(-r^{*}\right)} .
$$

This being an upper bound on $\left(-r^{*}\right)$, we will also need a lower bound: 
Proposition B.4. For $r^{*} \leq 0$,

$$
\left(-r^{*}\right) \geq \frac{(2 m)^{\frac{1}{n-2}}}{n-2} \log \left(\frac{\left(\frac{n}{2}\right)^{\frac{1}{n-2}}-1}{\left(\frac{n}{2}\right)^{\frac{1}{n-2}}+1} \frac{\frac{r}{n-2} \sqrt{2 m}+1}{\frac{r}{n-2}-1}\right) .
$$

Dyadic sequences. In our argument, Section $5 \mathrm{C}$ in particular, we construct a hierarchy of dyadic sequences, beginning with a sequence of real numbers $\left(\tau_{j}\right)_{j \in \mathbb{N}}$ where $\tau_{1}>0$ and $\tau_{j+1}=2 \tau_{j}(j \in \mathbb{N})$. We then obtain (by the mean value theorem of integration) a sequence $\left(\tau_{j}^{\prime}\right)_{j \in \mathbb{N}}$ with $\tau_{j}^{\prime}$ in the interval $\left(\tau_{j}, \tau_{j+1}\right)$ of length $\tau_{j}$ for all $j \in \mathbb{N}$. We then built up on these values another sequence $\left(\tau_{j}^{\prime \prime}\right)_{j \in \mathbb{N}}$ which takes values (as selected by the mean value theorem) in the intervals $\left(\tau_{2 j-1}^{\prime}, \tau_{2 j+1}^{\prime}\right) \ni \tau_{j}^{\prime \prime}$; note that their length is at least $\tau_{2 j+1}^{\prime}-\tau_{2 j-1}^{\prime} \geq \tau_{2 j+1}-\tau_{2 j}=\tau_{2 j}$. In the same fashion the sequence $\left(\tau_{j}^{\prime \prime \prime}\right)_{j \in \mathbb{N}}$ is built upon $\left(\tau_{j}^{\prime \prime}\right)_{j \in \mathbb{N}}$, etc.

\section{Appendix C: Boundary integrals and Hardy inequalities}

In this appendix we prove appropriate Hardy inequalities that are needed in our argument to estimate boundary terms that typically arise in the energy identities.

$X$-type currents. Let $X=f\left(r^{*}\right) \frac{\partial}{\partial r^{*}}$ and recall the modification (4-14).

Proposition C.1 (boundary terms near null infinity). Let $f=\mathcal{O}(1), f^{\prime}=\mathcal{O}\left(\frac{1}{r}\right)$, and $f^{\prime \prime}=\mathcal{O}\left(\frac{1}{r^{2}}\right)$. Then there exists a constant $C(n, m)$ such that

$$
\int_{\partial \mathcal{D}_{\tau_{1}}^{\tau_{2}} \backslash\{r=R\}}{ }^{*} J^{X, 1} \leq C(n, m) \int_{\Sigma_{\tau_{1}}}\left(J^{T}(\phi), n\right) .
$$

Proof. For the boundary integrals on the null segments $u^{*}=\tau_{1}, \tau_{2}$ we find

$$
\begin{aligned}
& \left|\int_{R^{*}+\tau_{i}}^{\infty} \mathrm{d} v^{*} \int_{\mathbb{S}^{n-1}} \mathrm{~d} \mu_{\dot{\gamma}_{n-1}} g\left(J^{X, 1}, \frac{\partial}{\partial v^{*}}\right) r^{n-1}\right| \\
& \quad \leq C(n) \int_{R^{*}+\tau_{i}}^{\infty} \mathrm{d} v^{*} \int_{\mathbb{S}^{n-1}} \mathrm{~d} \mu_{\dot{\gamma}_{n-1}} r^{n-1}\left\{\left(\frac{\partial \phi}{\partial v^{*}}\right)^{2}+|\not \varnothing \phi|^{2}+\left[\frac{|f|}{r^{2}}+\frac{\left|f^{\prime}\right|}{r}+\left|f^{\prime}\right|^{2}+\left|f^{\prime \prime}\right|\right] \phi^{2}\right\},
\end{aligned}
$$

and, in view of the Hardy inequality Lemma C.2,

$$
\left.\int_{R^{*}+\tau_{i}}^{\infty} \mathrm{d} v^{*} \int_{\mathbb{S}^{n-1}} \mathrm{~d} \mu_{\dot{\gamma}_{n-1}} \frac{1}{r^{2}} \phi^{2} r^{n-1}\right|_{u^{*}=\tau_{i}} \leq C(n, m) \int_{\Sigma_{\tau_{i}}}\left(J^{T}(\phi), n\right) ;
$$

note that the corresponding zero order terms vanish at future null infinity; cf. Remark C.3. Then (C-1) follows from the energy identity for $T$ on ${ }^{R} \mathcal{D}_{\tau_{1}}^{\tau_{2}}$.

Lemma C.2 (Hardy inequality). Let $\phi \in \mathrm{C}^{1}([a, \infty)), a>0$, with $|\phi(a)|<\infty$ and

$$
\lim _{x \rightarrow \infty} x^{\frac{n-2}{2}} \phi(x)=0 .
$$

Then a constant $C(n)>0$ exists such that

$$
\int_{a}^{\infty} \frac{1}{x^{2}} \phi^{2}(x) x^{n-1} \mathrm{~d} x \leq C(n) \int_{a}^{\infty}\left(\frac{\mathrm{d} \phi}{\mathrm{d} x}\right)^{2} x^{n-1} \mathrm{~d} x .
$$


Proof. This is a consequence of the Cauchy-Schwarz inequality; after integration by parts

$$
\int_{a}^{\infty} \frac{1}{x^{2}} \phi^{2}(x) x^{n-1} \mathrm{~d} x=\int_{a}^{\infty} g^{\prime}(x) \phi^{2}(x) \mathrm{d} x
$$

with

$$
g(x)=\int_{a}^{x} y^{n-3} \mathrm{~d} y .
$$

Remark C.3. The conditions of Lemma C.2 on $\phi$ are in fact satisfied for any solution of the wave equation (1-1). By a density argument we may assume without loss of generality that the initial data is compactly supported. Then for a fixed $\tau$, and $v^{*}$ large enough, $\phi\left(\tau, v^{*}\right)=0$, and for $u^{*} \geq \tau$,

Thus

$$
\phi\left(u^{*}, v^{*}\right)=\int_{\tau}^{u^{*}} \frac{\partial \phi}{\partial u^{*}} \mathrm{~d} u^{*} .
$$

$$
\phi\left(u^{*}, v^{*}\right) \leq\left(\int_{\tau}^{u^{*}}\left(\frac{\partial \phi}{\partial u^{*}}\right)^{2} r^{n-1} \mathrm{~d} u^{*}\right)^{\frac{1}{2}}\left(\int_{\tau}^{u^{*}} \frac{1}{r^{n-1}} \mathrm{~d} u^{*}\right)^{\frac{1}{2}}
$$

On one hand,

$$
\int_{\tau}^{u^{*}} \int_{\mathbb{S}^{n-1}}\left(\frac{\partial \phi}{\partial u^{*}}\right)^{2} r^{n-1} \mathrm{~d} \mu_{\dot{\gamma}_{n-1}} \mathrm{~d} u^{*} \leq \int_{\Sigma_{\tau}}\left(J^{T}(\phi), n\right)<\infty,
$$

whereas on the other hand,

$$
\begin{aligned}
\int_{\tau}^{u^{*}} \frac{1}{r^{n-1}} \mathrm{~d} u^{*} & =\frac{1}{n-2} \int_{\tau}^{u^{*}}\left(1-\frac{2 m}{r^{n-2}}\right)^{-1} \frac{\partial}{\partial u^{*}}\left(\frac{1}{r^{n-2}}\right) \mathrm{d} u^{*} \\
& \leq \frac{1}{n-2}\left(1-\frac{2 m}{R^{n-2}}\right)^{-1}\left(1-\left(\frac{r\left(u^{*}, v^{*}\right)}{r\left(\tau, v^{*}\right)}\right)^{n-2}\right) \frac{1}{r^{n-2}}
\end{aligned}
$$

if we restrict $u^{*} \geq \tau$ to $r\left(u^{*}, v^{*}\right) \geq R$. Hence

$$
\lim _{v^{*} \rightarrow \infty} r^{\frac{n-2}{2}} \phi=0 .
$$

Instead of (C-5), which requires (C-4), one can prove the corresponding Hardy inequality for finite intervals:

Lemma C.4 (Hardy inequality for finite intervals). Let $0<a<b$, and $\phi \in \mathrm{C}^{1}((a, b))$. Then

$$
\frac{1}{2} \int_{a}^{b} \frac{1}{x^{2}} \phi^{2}(x) x^{n-1} \mathrm{~d} x \leq \frac{1}{n-2} b^{n-2} \phi^{2}(b)+2\left(\frac{2}{n-2}\right)^{2} \int_{a}^{b}\left(\frac{\mathrm{d} \phi}{\mathrm{d} x}\right)^{2} x^{n-1} \mathrm{~d} x .
$$

Proof. Let

$$
g(x)=\int_{a}^{x} y^{n-3} \mathrm{~d} y=\left.\frac{1}{n-2} y^{n-2}\right|_{a} ^{x} .
$$

Then, by integration by parts and using Cauchy's inequality,

$$
\begin{aligned}
\int_{a}^{b} \frac{1}{x^{2}} \phi^{2}(x) x^{n-1} \mathrm{~d} x & =\left.g \phi^{2}\right|_{a} ^{b}-\int_{a}^{b} g(x) 2 \phi(x) \frac{\mathrm{d} \phi}{\mathrm{d} x} \mathrm{~d} x \\
& \leq g(b) \phi^{2}(b)+2 \epsilon \int_{a}^{b} \frac{1}{x^{2}} \phi^{2}(x) x^{n-1} \mathrm{~d} x+\frac{1}{2 \epsilon} \int_{a}^{b} \frac{g(x)^{2}}{x^{n-3}}\left(\frac{\mathrm{d} \phi}{\mathrm{d} x}\right)^{2} \mathrm{~d} x,
\end{aligned}
$$


where $\epsilon>0$; (C-6) follows for $\epsilon=\frac{1}{4}$ because

$$
g(b) \leq \frac{1}{n-2} b^{n-2}, \quad \frac{g(x)^{2}}{x^{n-3}} \leq \frac{2}{n-2}\left(1+\left(\frac{a}{x}\right)^{2(n-2)}\right) x^{n-1} .
$$

Recall the domain (4-103); by using Lemma C.4 instead of Lemma C.2 we can prove the following refinement of Proposition C.1 to bounded domains:

Proposition C.5 (boundary terms on bounded domains). Let $f=\mathcal{O}(1), f^{\prime}=\mathcal{O}\left(\frac{1}{r}\right)$, and $f^{\prime \prime}=\mathcal{O}\left(\frac{1}{r^{2}}\right)$. Then there exists a constant $C(n, m)$ such that

$$
\int_{\partial^{R} \mathcal{L}_{\tau_{1}}^{\tau_{2}} \backslash\{r=R\}}{ }^{*} J^{X, 1} \leq C(n, m)\left\{\int_{\Sigma_{\tau_{1}}^{\tau_{2}}}\left(J^{T}(\phi), n\right)+\left.\int_{\mathbb{S}^{n-1}} \mathrm{~d} \mu_{\dot{\gamma}_{n-1}} r^{n-2} \phi^{2}\right|_{\left(u^{*}=\tau_{1}, v^{*}=R^{*}+\tau_{2}\right)}\right\} .
$$

Recall the domain (4-2).

Proposition C.6 (boundary terms near the event horizon). Let $f=\mathcal{O}(1), f^{\prime}=\mathcal{O}\left(\frac{1}{\left|r^{*}\right|^{4}}\right)$, and $f^{\prime \prime}=$ $\mathcal{O}\left(\frac{1}{\left|r^{*}\right|^{5}}\right)$, and

$$
\pi_{l} \phi=0 \quad(0 \leq l<L),
$$

for some $L \in \mathbb{N}$. Then there exists a constant $C(n, m, L)$ such that

$$
\int_{\partial \mathcal{R}_{r_{0}, r_{1}}^{\infty}\left(t_{0}\right)}{ }^{*} J^{X, 1} \leq C(n, m, L) \int_{\Sigma_{\tau_{0}}}\left(J^{T}(\phi), n\right),
$$

where $\tau_{0}=\frac{1}{2}\left(t_{0}-r_{1}^{*}\right)$.

The proof is given in Section 4D.1 in the special case $f=f_{\gamma, \alpha}$ using the following lemma.

Lemma C.7 (Hardy inequality). Let $a>0, \phi \in \mathrm{C}^{1}([a, \infty))$ with

$$
\lim _{x \rightarrow \infty}|\phi(x)|<\infty
$$

Then

$$
\int_{a}^{\infty} \frac{1}{1+x^{2}} \phi^{2}(x) \mathrm{d} x \leq 8 \frac{1+a^{2}}{a^{2}} \int_{a}^{\infty}\left(\frac{\mathrm{d} \phi}{\mathrm{d} x}\right)^{2} \mathrm{~d} x+2 \pi \int_{a}^{a+1}\left\{\phi^{2}+\left(\frac{\mathrm{d} \phi}{\mathrm{d} x}\right)^{2}\right\} \mathrm{d} x .
$$

Proof. Let us first assume that $\phi(a)=0$. Define

$$
g(x)=-\int_{x}^{\infty} \frac{1}{1+y^{2}} \mathrm{~d} y .
$$

Then

$$
\begin{aligned}
\int_{a}^{\infty} \frac{1}{1+x^{2}} \phi^{2}(x) \mathrm{d} x & =\int_{a}^{\infty} g^{\prime}(x) \phi^{2}(x) \mathrm{d} x=\left.g(x) \phi^{2}(x)\right|_{a} ^{\infty}-2 \int_{a}^{\infty} g(x) \phi(x) \frac{\mathrm{d} \phi}{\mathrm{d} x} \mathrm{~d} x \\
& \leq 2\left(\int_{a}^{\infty} \frac{g(x)^{2}}{g^{\prime}(x)}\left(\frac{\mathrm{d} \phi}{\mathrm{d} x}\right)^{2} \mathrm{~d} x\right)^{\frac{1}{2}}\left(\int_{a}^{\infty} g^{\prime}(x) \phi^{2}(x) \mathrm{d} x\right)^{\frac{1}{2}}
\end{aligned}
$$


Since $|g(x)| \leq \frac{1}{x}$ we have

$$
\frac{g(x)^{2}}{g^{\prime}(x)} \leq \frac{1+x^{2}}{x^{2}} \leq \frac{1+a^{2}}{a^{2}}
$$

and therefore

$$
\int_{a}^{\infty} \frac{1}{1+x^{2}} \phi^{2}(x) \mathrm{d} x \leq 4 \int_{a}^{\infty} \frac{g(x)^{2}}{g^{\prime}(x)}\left(\frac{\mathrm{d} \phi}{\mathrm{d} x}\right)^{2} \mathrm{~d} x \leq 4 \frac{1+a^{2}}{a^{2}} \int_{a}^{\infty}\left(\frac{\mathrm{d} \phi}{\mathrm{d} x}\right)^{2} \mathrm{~d} x .
$$

Without the assumption $\phi(a)=0$ this applied to the function $\phi(x)-\phi(a)$ yields

$$
\begin{aligned}
\int_{a}^{\infty} \frac{1}{1+x^{2}} \phi^{2}(x) \mathrm{d} x & \leq 2 \int_{a}^{\infty} \frac{1}{1+x^{2}}(\phi(x)-\phi(a))^{2} \mathrm{~d} x+2 \int_{a}^{\infty} \frac{1}{1+x^{2}} \phi(a)^{2} \mathrm{~d} x \\
& \leq 8 \frac{1+a^{2}}{a^{2}} \int_{a}^{\infty}\left(\frac{\mathrm{d} \phi}{\mathrm{d} x}\right)^{2} \mathrm{~d} x+\pi \phi(a)^{2}
\end{aligned}
$$

We conclude the proof with the following pointwise bound: on one hand, for some $a^{\prime} \in(a, a+1)$,

$$
\int_{a}^{a+1} \phi(x)^{2} \mathrm{~d} x=\phi\left(a^{\prime}\right)^{2}
$$

and on the other hand,

$$
\phi\left(a^{\prime}\right)^{2}-\phi(a)^{2}=\int_{a}^{a^{\prime}} \frac{\mathrm{d}}{\mathrm{d} x} \phi(x)^{2} \mathrm{~d} x \leq \int_{a}^{a^{\prime}}\left\{\phi(x)^{2}+\left(\frac{\mathrm{d} \phi}{\mathrm{d} x}\right)^{2}\right\} \mathrm{d} x .
$$

Hence

$$
\phi(a)^{2} \leq \int_{a}^{a^{\prime}}\left\{\phi(x)^{2}+\left(\frac{\mathrm{d} \phi}{\mathrm{d} x}\right)^{2}\right\} \mathrm{d} x+\int_{a}^{a+1} \phi(x)^{2} \mathrm{~d} x \leq 2 \int_{a}^{a+1}\left\{\phi(x)^{2}+\left(\frac{\mathrm{d} \phi}{\mathrm{d} x}\right)^{2}\right\} \mathrm{d} x .
$$

Auxiliary currents. We have the same results for auxiliary currents of the form

$$
J_{\mu}^{\text {aux }}=\frac{1}{2} h(r) \partial_{\mu}\left(\phi^{2}\right) .
$$

Proposition C.8. Let $h=\mathcal{O}\left(\frac{1}{r}\right)$. Then there exists a constant $C(n, m)$ such that

$$
\int_{\partial^{R} \mathcal{D}_{\tau_{1}}^{\tau_{2}} \backslash\{r=R\}}{ }^{*} J^{\text {aux }} \leq C(n, m) \int_{\Sigma_{\tau_{1}}}\left(J^{T}(\phi), n\right),
$$

and moreover, for a constant $C(n, m)$, we have the refinement

$$
\int_{\partial^{R} \mathcal{L}_{\tau_{1}}^{\tau_{2}} \backslash\{r=R\}}{ }^{*} J^{\mathrm{aux}} \leq C(n, m)\left\{\int_{\Sigma_{\tau_{1}}^{\tau_{2}}}\left(J^{T}(\phi), n\right)+\left.\int_{\mathbb{S}^{n-1}} \mathrm{~d} \mu_{\dot{\gamma}_{n-1}} r^{n-2} \phi^{2}\right|_{\left(\tau_{1}, R^{*}+\tau_{2}\right)}\right\} .
$$

Proof. Note that here, in comparison to the proof of Proposition C.1,

$$
\left|g\left(J^{\text {aux }}, \frac{\partial}{\partial v^{*}}\right)\right| \leq h^{2} \phi^{2}+\left(\frac{\partial \phi}{\partial v^{*}}\right)^{2} .
$$


Proposition C.9. Let $h=\mathcal{O}\left(\frac{1}{\left|r^{*}\right|}\right)$. Then there exists a constant $C(n, m)$ such that

where $\tau_{0}=\frac{1}{2}\left(t_{0}-r_{1}^{*}\right)$.

$$
\int_{\partial \mathcal{R}_{r_{0}, r_{1}}^{\infty}\left(t_{0}\right)} * J^{\mathrm{aux}} \leq C(n, m) \int_{\Sigma_{\tau_{0}}}\left(J^{T}(\phi), n\right),
$$

Remark C.10. In view of Proposition B.3, the function $h=\frac{1}{r}\left(1-\frac{2 m}{r^{n-2}}\right)$ satisfies the assumption of the proposition.

\section{Acknowledgements}

The author would like to thank Mihalis Dafermos for suggesting this problem and for his support and encouragement. The author also thanks the UK Engineering and Physical Sciences Research Council and the Cambridge European Trust as well as the European Research Council for their financial support.

\section{References}

[Alinhac 2009] S. Alinhac, "Energy multipliers for perturbations of the Schwarzschild metric", Comm. Math. Phys. 288:1 (2009), 199-224. MR 2010b:58038 Zbl 1196.53053

[Andersson and Blue 2009] L. Andersson and P. Blue, "Hidden symmetries and decay for the wave equation on the Kerr spacetime”, preprint, 2009. arXiv 0908.2265

[Aretakis 2011] S. Aretakis, "Stability and instability of extreme Reissner-Nordström black hole spacetimes for linear scalar perturbations, I", Comm. Math. Phys. 307:1 (2011), 17-63. MR 2835872 Zbl 1229.85002

[Blue and Soffer 2003] P. Blue and A. Soffer, "Semilinear wave equations on the Schwarzschild manifold, I: Local decay estimates", Adv. Differential Equations 8:5 (2003), 595-614. MR 2004k:58046 Zbl 1044.58033

[Blue and Sterbenz 2006] P. Blue and J. Sterbenz, "Uniform decay of local energy and the semi-linear wave equation on Schwarzschild space”, Comm. Math. Phys. 268:2 (2006), 481-504. MR 2007i:58037 Zbl 1123.58018

[Ching et al. 1995] E. S. C. Ching, P. T. Leung, W. M. Suen, and K. Young, "Wave propagation in gravitational systems: Late time behavior", Phys. Rev. D 52 (1995), 2118-2132.

[Choquet-Bruhat et al. 2006] Y. Choquet-Bruhat, P. T. Chruściel, and J. Loizelet, "Global solutions of the Einstein-Maxwell equations in higher dimensions", Classical Quantum Gravity 23:24 (2006), 7383-7394. MR 2008i:83022 Zbl 1117.83024

[Christodoulou 1995] D. Christodoulou, "Self-gravitating relativistic fluids: A two-phase model", Arch. Rational Mech. Anal. 130:4 (1995), 343-400. MR 96i:83046 Zbl 0841.76097

[Christodoulou and Klainerman 1990] D. Christodoulou and S. Klainerman, "Asymptotic properties of linear field equations in Minkowski space”, Comm. Pure Appl. Math. 43:2 (1990), 137-199. MR 91a:58202 Zbl 0715.35076

[Christodoulou and Klainerman 1993] D. Christodoulou and S. Klainerman, The global nonlinear stability of the Minkowski space, Princeton Mathematical Series 41, Princeton University Press, 1993. MR 95k:83006 Zbl 0827.53055

[Dafermos and Holzegel 2006] M. Dafermos and G. Holzegel, "On the nonlinear stability of higher dimensional triaxial Bianchi-IX black holes”, Adv. Theor. Math. Phys. 10:4 (2006), 503-523. MR 2007h:83039 Zbl 1106.83014

[Dafermos and Rodnianski 2007] M. Dafermos and I. Rodnianski, "A note on energy currents and decay for the wave equation on a Schwarzschild background", preprint, 2007. arXiv 0710.0171v1

[Dafermos and Rodnianski 2008] M. Dafermos and I. Rodnianski, "Lectures on black holes and linear waves", preprint, 2008. arXiv 0811.0354

[Dafermos and Rodnianski 2009a] M. Dafermos and I. Rodnianski, "The black hole stability problem", Oberwolfach Rep. 6 (2009), 2589-2594.

[Dafermos and Rodnianski 2009b] M. Dafermos and I. Rodnianski, "The red-shift effect and radiation decay on black hole spacetimes”, Comm. Pure Appl. Math. 62:7 (2009), 859-919. MR 2011b:83059 Zbl 1169.83008 
[Dafermos and Rodnianski 2010] M. Dafermos and I. Rodnianski, "A new physical-space approach to decay for the wave equation with applications to black hole spacetimes", pp. 421-432 in XVIth international congress on mathematical physics, edited by P. Exner, World Scientific, Hackensack, NJ, 2010. MR 2012e:58051 Zbl 1211.83019

[Dafermos and Rodnianski 2011] M. Dafermos and I. Rodnianski, "A proof of the uniform boundedness of solutions to the wave equation on slowly rotating Kerr backgrounds", Invent. Math. 185:3 (2011), 467-559. MR 2827094 Zbl 1226.83029

[Dafermos and Rodnianski 2012] M. Dafermos and I. Rodnianski, "The black hole stability problem for linear scalar perturbations", pp. 132-189 in The twelfth Marcel Grossmann meeting on general relativity: Recent developments in theoretical and experimental general relativity, astrophysics, and relativistic field theories (Paris, 2009), vol. 1, edited by R. Jantzen et al., World Scientifc, Singapore, 2012.

[Donninger et al. 2012] R. Donninger, W. Schlag, and A. Soffer, "On pointwise decay of linear waves on a Schwarzschild black hole background", Comm. Math. Phys. 309:1 (2012), 51-86. MR 2864787 Zbl 1242.83054

[Emparan and Reall 2008] R. Emparan and H. S. Reall, "Black holes in higher dimensions", Living Rev. Relativ. 11 (2008), Article no. 2008-6. Zbl 1166.83002

[Hawking and Ellis 1973] S. W. Hawking and G. F. R. Ellis, The large scale structure of space-time, Cambridge Monographs on Mathematical Physics 1, Cambridge University Press, London, 1973. MR 54 \#12154 Zbl 0265.53054

[Holzegel 2010] G. Holzegel, "Stability and decay rates for the five-dimensional Schwarzschild metric under biaxial perturbations", Adv. Theor. Math. Phys. 14:5 (2010), 1245-1372. MR 2012j:83050 Zbl 1243.83023

[Laul and Metcalfe 2012] P. Laul and J. Metcalfe, "Localized energy estimates for wave equations on high-dimensional Schwarzschild space-times", Proc. Amer. Math. Soc. 140:9 (2012), 3247-3262. MR 2917097

[Luk 2010] J. Luk, "Improved decay for solutions to the linear wave equation on a Schwarzschild black hole", Ann. Henri Poincaré 11:5 (2010), 805-880. MR 2012k:58045 Zbl 1208.83068

[Marzuola et al. 2010] J. Marzuola, J. Metcalfe, D. Tataru, and M. Tohaneanu, "Strichartz estimates on Schwarzschild black hole backgrounds", Comm. Math. Phys. 293:1 (2010), 37-83. MR 2010m:58043 Zbl 1202.35327

[Morawetz 1962] C. S. Morawetz, "The limiting amplitude principle”, Comm. Pure Appl. Math. 15 (1962), 349-361. MR 27 \#1696 Zbl 0196.41202

[Schlue 2010] V. Schlue, "Linear waves on higher dimensional Schwarzschild black holes", Smith-Rayleigh-Knight Prize Essay, University of Cambridge, 2010.

[Schlue 2012] V. Schlue, Linear waves on higher dimensional Schwarzschild black holes and Schwarzschild de Sitter spacetimes, Ph.D. thesis, University of Cambridge, 2012, http://www.dspace.cam.ac.uk/handle/1810/243640.

[Tangherlini 1963] F. R. Tangherlini, "Schwarzschild field in $n$ dimensions and the dimensionality of space problem", Nuovo Cimento (10) 27 (1963), 636-651. MR 26 \#5955 Zbl 0114.21302

[Tataru 2010] D. Tataru, "Local decay of waves on asymptotically flat stationary space-times", preprint, 2010. arXiv 0910.5290v2

Received 28 Mar 2011. Revised 4 Jun 2012. Accepted 20 Dec 2012.

VOLKER SCHLUE: vschlue@math.utoronto.ca

Department of Pure Mathematics and Mathematical Statistics, University of Cambridge, Cambridge, CB3 OWB, United Kingdom

Current address: Department of Mathematics, University of Toronto, 40 St. George Street, Room 6120, Toronto, ON M5S 2E4, Canada 


\title{
Analysis \& PDE
}

\author{
msp.org/apde
}

\section{EDITORS}

EDITOR-IN-CHIEF

\author{
Maciej Zworski \\ zworski@math.berkeley.edu \\ University of California \\ Berkeley, USA
}

BOARD OF EDITORS

\begin{abstract}
Michael Aizenman
Luis A. Caffarelli

Michael Christ

Ursula Hamenstaedt

Vaughan Jones

Izabella Laba

László Lempert

Frank Merle

Werner Müller

Gilles Pisier

Igor Rodnianski

Sylvia Serfaty

Terence Tao

Gunther Uhlmann

Dan Virgil Voiculescu

Princeton University, USA aizenman@math.princeton.edu

University of Texas, USA caffarel@math.utexas.edu

University of California, Berkeley, USA mchrist@math.berkeley.edu

Universität Bonn, Germany ursula@math.uni-bonn.de

University of California, Berkeley, USA vfr@math.berkeley.edu

University of British Columbia, Canada ilaba@math.ubc.ca

Purdue University, USA lempert@math.purdue.edu

Université de Cergy-Pontoise, France Frank.Merle@u-cergy.fr

Universität Bonn, Germany mueller@math.uni-bonn.de

Texas A\&M University, and Paris 6 pisier@math.tamu.edu

Princeton University, USA irod@math.princeton.edu

New York University, USA serfaty@cims.nyu.edu

University of California, Los Angeles, USA tao@math.ucla.edu

University of Washington, USA gunther@math.washington.edu

University of California, Berkeley, USA dvv@math.berkeley.edu
\end{abstract}

Nicolas Burq Université Paris-Sud 11, France nicolas.burq@math.u-psud.fr

Sun-Yung Alice Chang Princeton University, USA chang@math.princeton.edu

Charles Fefferman Princeton University, USA cf@math.princeton.edu

Nigel Higson Pennsylvania State Univesity, USA higson@math.psu.edu

Herbert Koch Universität Bonn, Germany koch@math.uni-bonn.de

Gilles Lebeau Université de Nice Sophia Antipolis, France lebeau@unice.fr

Richard B. Melrose Massachussets Institute of Technology, USA rbm@math.mit.edu

William Minicozzi II

Johns Hopkins University, USA minicozz@math.jhu.edu

Yuval Peres University of California, Berkeley, USA peres@stat.berkeley.edu

Tristan Rivière ETH, Switzerland riviere@math.ethz.ch

Wilhelm Schlag University of Chicago, USA schlag@math.uchicago.edu

Yum-Tong Siu Harvard University, USA siu@math.harvard.edu

Michael E. Taylor Univ. of North Carolina, Chapel Hill, USA met@math.unc.edu

András Vasy Stanford University, USA andras@math.stanford.edu

Steven Zelditch Northwestern University, USA zelditch@math.northwestern.edu

PRODUCTION

production@msp.org

Silvio Levy, Scientific Editor

See inside back cover or msp.org/apde for submission instructions.

The subscription price for 2013 is US $\$ 160 /$ year for the electronic version, and \$310/year (+\$35, if shipping outside the US) for print and electronic. Subscriptions, requests for back issues from the last three years and changes of subscribers address should be sent to MSP.

Analysis \& PDE (ISSN 1948-206X electronic, 2157-5045 printed) at Mathematical Sciences Publishers, 798 Evans Hall \#3840, c/o University of California, Berkeley, CA 94720-3840, is published continuously online. Periodical rate postage paid at Berkeley, CA 94704, and additional mailing offices.

APDE peer review and production are managed by EditFLow ${ }^{\circledR}$ from Mathematical Sciences Publishers.

\section{PUBLISHED BY}

mathematical sciences publishers

nonprofit scientific publishing

http://msp.org/

(C) 2013 Mathematical Sciences Publishers 


\section{ANALYSIS \& PDE Volume $6 \quad$ No. $3 \quad 2013$}

Decay of linear waves on higher-dimensional Schwarzschild black holes VOLKER SCHLUE

Conditional global regularity of Schrödinger maps: subthreshold dispersed energy PAUL SMITH

Bilinear dispersive estimates via space-time resonances, I: the one-dimensional case FRÉDÉRIC BERNICOT and PIERRE GERMAIN

Smoothing and global attractors for the Zakharov system on the torus 